Supporting Information

\title{
Dynamic Kinetic Resolution of Biaryl Lactones via a Chiral Bifunctional Amine Thiourea Catalyzed Highly atropo-Enantioselective Transesterification
}

\author{
Chenguang $\mathrm{Yu}^{\dagger}{ }^{\dagger}$ He Huang, ${ }^{\dagger}$ Xiangmin $\mathrm{Li}^{\dagger,}{ }^{\dagger,}$ Yueteng Zhang ${ }^{\dagger}$ and Wei Wang ${ }^{\dagger, *^{*}}$ \\ ${ }^{\dagger}$ Department of Chemistry and Chemical Biology, University of New Mexico, Albuqueruqe, NM 87131- \\ 0001 \\ ${ }^{\ddagger}$ School of Pharmacy, East China University of Science \& Technology, Shanghai 200237, China
}

\section{Table of Contents}

\begin{tabular}{|ll|c|}
\hline 1. & General Information & $\mathrm{S} 2$ \\
\hline 2. & Synthesis of Substrates and Characterization Data & $\mathrm{S} 2$ \\
\hline 3. & General Procedures for the atropo-Enantioselective Esterification and Characterization Data & $\mathrm{S} 5$ \\
\hline 4. & Original ${ }^{1} \mathrm{H}$ and ${ }^{13} \mathrm{C}$ NMR Spectra and Chiral HPLC analysis trace & $\mathrm{S} 21$ \\
\hline
\end{tabular}


1. General Information: Commercial reagents were used as received, unless otherwise stated. Merck 60 silica gel was used for chromatography, and Whatman silica gel plates with fluorescence $\mathrm{F}_{254}$ indicator were used for thin-layer chromatography (TLC) analysis. ${ }^{1} \mathrm{H}$ and ${ }^{13} \mathrm{C}$ NMR spectra were recorded on Bruker Avance 300. Chemical shifts in ${ }^{1} \mathrm{H}$ NMR spectra are reported in parts per million (ppm) relative to residual chloroform $(7.26 \mathrm{ppm})$ as internal standards. Data for ${ }^{1} \mathrm{H}$ are reported as follows: chemical shift $(\mathrm{ppm})$, and multiplicity $(\mathrm{s}=$ singlet, $\mathrm{d}=$ doublet, $\mathrm{t}=$ triplet, $\mathrm{q}=$ quartet, quint $=$ quintet, $\mathrm{m}=$ multiplet). Data for ${ }^{13} \mathrm{C}$ NMR are reported as ppm. ${ }^{13} \mathrm{C}$ NMR chemical shifts are reported in ppm relative to the central peak of $\mathrm{CDCl}_{3}(77.16 \mathrm{ppm})$ as internal standards.

\section{Synthesis of Substrates and Characterization Data:}

General Precedure for the synthesis of compounds 1 using 1a as example:
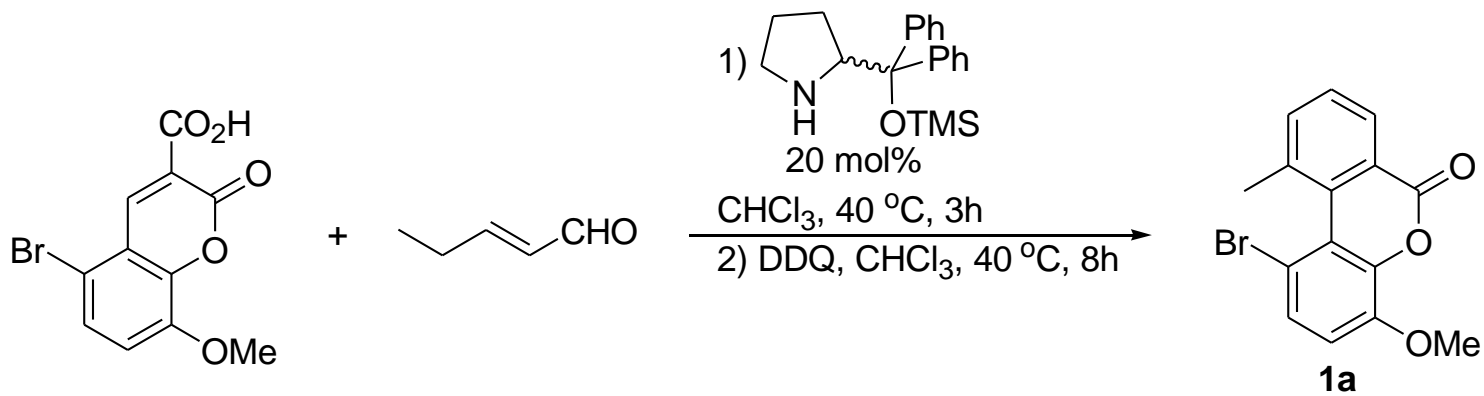

A mixture of 5-bromo-8-methoxy-2-oxo-2H-chromene-3-carboxylic acid (1 mmol, $299 \mathrm{mg})$, trans-2pentenal $(1.5 \mathrm{mmol}, 147 \mu \mathrm{L})$ and catalyst $(0.2 \mathrm{mmol}, 65 \mathrm{mg})$ was stirred in $5 \mathrm{~mL}$ chloroform at $40^{\circ} \mathrm{C}$ for $3 \mathrm{~h}$. Then, $2 \mathrm{mmol}$ (454 mg) of 2,3-dichloro-5,6-dicyano-1,4-benzoquinone (DDQ) was added and stirred for $8 \mathrm{~h}$ at $40^{\circ} \mathrm{C}$. $1 \mathrm{~g}$ of silica gel was added and the sample was dried by vacuum. The sample was subjected to flash column chromatography on silica gel (eluent: hexanes/ethyl ether $=15: 1-10: 1)$ to afford $1 \mathrm{a}$ in $70 \%$ yield.<smiles>COc1ccc(Br)c2c1oc(=O)c1cccc(C)c12</smiles>

1-Bromo-4-methoxy-10-methyl-6H-benzo[c]chromen-6-one (1a): White solid, 70\% yield; ${ }^{1} \mathrm{H}-\mathrm{NMR}$ $\left(300 \mathrm{MHz}, \mathrm{CDCl}_{3}\right): \delta=8.18\left(\mathrm{dd}, 1 \mathrm{H}, J_{1}=0.9 \mathrm{~Hz}, J_{2}=7.5 \mathrm{~Hz}\right), 7.67(\mathrm{~d}, 1 \mathrm{H}, J=6.9 \mathrm{~Hz}), 7.54(\mathrm{t}, 1 \mathrm{H}, J=$ $7.5 \mathrm{~Hz}), 7.48(\mathrm{~d}, 1 \mathrm{H}, J=8.7 \mathrm{~Hz}), 6.94(\mathrm{~d}, 1 \mathrm{H}, J=8.7 \mathrm{~Hz}), 3.97(\mathrm{~s}, 3 \mathrm{H}), 2.67(\mathrm{~s}, 3 \mathrm{H}) .{ }^{13} \mathrm{C}$ NMR $(75 \mathrm{MHz}$, $\mathrm{CDCl}_{3}$, TMS): 160.8, 147.4, 137.1, 136.5, 134.0, 129.2, 128.7, 128.2, 127.2, 124.6, 120.9, 112.7, 110.8, 56.5, 23.4. 
<smiles>COc1ccc(Br)c2c1oc(=O)c1cc(C)cc(C)c12</smiles>

1-Bromo-4-methoxy-8,10-dimethyl-6H-benzo[c]chromen-6-one (1b): White solid, 75\% yield; ${ }^{1} \mathrm{H}-$ NMR $\left(300 \mathrm{MHz}, \mathrm{CDCl}_{3}\right): \delta=7.99(\mathrm{~s}, 1 \mathrm{H}), 7.45-7.74(\mathrm{~m}, 2 \mathrm{H}), 6.89(\mathrm{~d}, 1 \mathrm{H}, J=9.0 \mathrm{~Hz}), 3.96(\mathrm{~s}, 3 \mathrm{H})$, 2.63 (s, 3H), 2.46 (s, 3H). ${ }^{13} \mathrm{C}$ NMR (75 MHz, $\left.\mathrm{CDCl}_{3}, \mathrm{TMS}\right): 161.1,147.3,141.4,139.1,138.2,136.4$, 131.6, 129.2, 127.4, 124.4, 121.0, 112.4, 110.6, 56.5, 23.3, 21.0.<smiles>CCc1cccc2c(=O)oc3c(OC)ccc(Br)c3c12</smiles>

1-Bromo-10-ethyl-4-methoxy-6H-benzo[c]chromen-6-one (1c): White solid, 60\% yield; ${ }^{1} \mathrm{H}-\mathrm{NMR}$ $\left(300 \mathrm{MHz}, \mathrm{CDCl}_{3}\right): \delta=8.16(\mathrm{~d}, 1 \mathrm{H}, J=7.5 \mathrm{~Hz}), 7.75(\mathrm{~d}, 1 \mathrm{H}, J=7.8 \mathrm{~Hz}), 7.57(\mathrm{t}, 1 \mathrm{H}, J=7.8 \mathrm{~Hz}), 7.45$ $(\mathrm{d}, 1 \mathrm{H}, J=8.7 \mathrm{~Hz}), 6.90(\mathrm{~d}, 1 \mathrm{H}, J=9.0 \mathrm{~Hz}), 3.97(\mathrm{~s}, 3 \mathrm{H}), 3.21-3.31(\mathrm{~m}, 1 \mathrm{H}), 2.87-2.99(\mathrm{~m}, 1 \mathrm{H}), 1.02(\mathrm{t}$, $3 \mathrm{H}, J=7.5 \mathrm{~Hz}) .{ }^{13} \mathrm{C} \mathrm{NMR}\left(75 \mathrm{MHz}, \mathrm{CDCl}_{3}\right.$, TMS): 160.8, 147.4, 143.3, 141.5, 135.2, 132.9, 129.1, $127.1,124.3,120.8,112.5,111.0,56.4,29.0,16.9$.<smiles>COc1ccc(Br)c2c1oc(=O)c1cccc(Br)c12</smiles>

10-Benzyl-1-bromo-4-methoxy-6H-benzo[c]chromen-6-one (1d): White solid, 38\% yield; ${ }^{1} \mathrm{H}-\mathrm{NMR}$ $\left(300 \mathrm{MHz} \mathrm{CDCl}_{3}\right): \delta=8.18\left(\mathrm{dd}, 1 \mathrm{H}, J_{1}=1.2 \mathrm{~Hz}, J_{2}=7.5 \mathrm{~Hz}\right), 7.58(\mathrm{~d}, 1 \mathrm{H}, J=7.8 \mathrm{~Hz}), 7.46-7.51(\mathrm{~m}$, 2H), 7.10-7.17 (m, 3H), $6.94(\mathrm{~d}, 1 \mathrm{H}, J=8.7 \mathrm{~Hz}), 6.79(\mathrm{~d}, 2 \mathrm{H}, J=6.3 \mathrm{~Hz}), 4.36-4.58(\mathrm{~m}, 2 \mathrm{H}), 4.00(\mathrm{~s}$, $3 \mathrm{H}) .{ }^{13} \mathrm{C} \mathrm{NMR}\left(75 \mathrm{MHz}, \mathrm{CDCl}_{3}\right.$, TMS): 160.7, 147.5, 141.6, 141.5, 140.0, 137.3, 133.6, 129.3, 129.2, $128.7,128.4,127.7,126.2,124.2,120.6,112.7,110.9,56.4,42.0$.<smiles>COc1ccc(Br)c2c1oc(=O)c1cccc(C(F)F)c12</smiles>

1-Bromo-10-isopropyl-4-methoxy-6H-benzo[c]chromen-6-one (1e): White solid, 40\% yield; ${ }^{1} \mathrm{H}-$ $\operatorname{NMR}\left(300 \mathrm{MHz}, \mathrm{CDCl}_{3}\right): \delta=8.16\left(\mathrm{dd}, 1 \mathrm{H}, J_{1}=1.2 \mathrm{~Hz}, J_{2}=7.8 \mathrm{~Hz}\right), 8.16\left(\mathrm{dd}, 1 \mathrm{H}, J_{1}=1.2 \mathrm{~Hz}, J_{2}=\right.$ 
$7.8 \mathrm{~Hz}), 7.60(\mathrm{t}, 1 \mathrm{H}, J=7.8 \mathrm{~Hz}), 7.45(\mathrm{~d}, 1 \mathrm{H}, J=8.7 \mathrm{~Hz}), 6.89(\mathrm{~d}, 1 \mathrm{H}, J=9.0 \mathrm{~Hz}), 3.98(\mathrm{~s}, 3 \mathrm{H}), 3.75$ (quint, $1 \mathrm{H}, J=6.6 \mathrm{~Hz}), 1.64(\mathrm{~d}, 1 \mathrm{H}, J=6.6 \mathrm{~Hz}), 0.74(\mathrm{~d}, 1 \mathrm{H}, J=6.9 \mathrm{~Hz}) \cdot{ }^{13} \mathrm{C} \mathrm{NMR}\left(75 \mathrm{MHz}, \mathrm{CDCl}_{3}\right.$, TMS): 161.3, 145.7, 139.0, 135.8, 132.9, 131.3, 131.0, 128.3, 128.0, 127.3, 127.0, 126.3, 125.8, 124.3, 122.3, 115.3, 110.4, 23.5, 21.0.<smiles>COc1ccc(Br)c2c1oc(=O)c1ccc3c(c12)CCCC3</smiles>

1-Bromo-4-methoxy-9,10,11,12-tetrahydro-6H-naphtho[2,1-c]chromen-6-one (1f): White solid, $43 \%$ yield; ${ }^{1} \mathrm{H}-\mathrm{NMR}\left(300 \mathrm{MHz}, \mathrm{CDCl}_{3}\right): \delta=8.07(\mathrm{~d}, 1 \mathrm{H}, J=7.8 \mathrm{~Hz}), 7.43(\mathrm{~d}, 1 \mathrm{H}, J=8.7 \mathrm{~Hz}), 7.35(\mathrm{~d}, 1 \mathrm{H}$, $J=7.8 \mathrm{~Hz}), 6.88(\mathrm{~d}, 1 \mathrm{H}, J=8.7 \mathrm{~Hz}), 3.94(\mathrm{~s}, 3 \mathrm{H}), 3.40-3.48(\mathrm{~m}, 1 \mathrm{H}), 2.83-2.97(\mathrm{~m}, 2 \mathrm{H}), 2.51-2.56(\mathrm{~m}$, 1H), 1.88-2.01 (m, 2H), 1.71-1.75 (m, 1H), 1.18-1.31 (m, 1H). ${ }^{13} \mathrm{C}$ NMR (75 MHz, CDCl 3 , TMS): 161.0, 147.3, 146.7, 141.6, 138.1, 132.9, 129.8, 128.9, 126.7, 121.9, 120.8, 112.3, 110.9, 56.4, 30.2, 29.3, 21.8, 21.1.<smiles>Cc1cc(C)c2c(c1)c(=O)oc1c(Br)cc3ccccc3c12</smiles>

$1 \mathrm{~h}$

7-Bromo-1,3-dimethyl-5H-dibenzo[c,f]chromen-5-one (1h): White solid, 50\% yield; ${ }^{1} \mathrm{H}-\mathrm{NMR}$ (300 $\left.\mathrm{MHz}, \mathrm{CDCl}_{3}\right): \delta=8.12-8.19(\mathrm{~m}, 2 \mathrm{H}), 7.81(\mathrm{~d}, 1 \mathrm{H}, J=7.5 \mathrm{~Hz}), 7.80(\mathrm{~d}, 1 \mathrm{H}, J=8.1 \mathrm{~Hz}), 7.47-7.56(\mathrm{~m}$, $3 \mathrm{H}), 2.53(\mathrm{~s}, 3 \mathrm{H}), 2.27$ (s, 3H). ${ }^{13} \mathrm{C}$ NMR (75 MHz, $\mathrm{CDCl}_{3}$, TMS): 161.2, 145.6, 139.0, 138.9, 135.7, $132.9,131.2,131.0,128.2,128.0,127.3,127.0,126.2,125.8,124.2,115.2,110.4,23.5,21.0$.<smiles>Cc1cc(C)c2c(c1)c(=O)oc1ccc3ccccc3c12</smiles>

$1 \mathrm{i}$

1,3-Dimethyl-5H-dibenzo[c,f]chromen-5-one (1i): White solid, 55\% yield; ${ }^{1} \mathrm{H}-\mathrm{NMR}(300 \mathrm{MHz}$, $\left.\mathrm{CDCl}_{3}\right): \delta=8.13(\mathrm{~s}, 1 \mathrm{H}), 7.90-7.94(\mathrm{~m}, 2 \mathrm{H}), 7.78(\mathrm{~d}, 1 \mathrm{H}, J=8.1 \mathrm{~Hz}), 7.50-7.56(\mathrm{~m}, 4 \mathrm{H}), 2.53(\mathrm{~s}, 3 \mathrm{H})$, 2.32 (s, 3H). ${ }^{13} \mathrm{C}$ NMR (75 MHz, $\mathrm{CDCl}_{3}$, TMS): 162.0, 149.2, 138.7, 138.0, 135.3, 131.6, 130.7, 130.3, $129.0,128.1,127.8,126.8,125.9,124.9,124.1,116.8,113.4,23.4,20.9$. 


\section{General Procedures for the atropo-Enantioselective Esterification and Characterization Data:}

General Procedure for atropo-enantioselective transesterification of biaryl lactones with alcohols (Scheme 2, 3a): A mixture of biaryl lactones $1 \mathbf{a}(0.1 \mathrm{mmol}, 31.9 \mathrm{mg})$, 4-nitrobenzyl alcohol 2a $(0.12$ $\mathrm{mmol}, 18.4 \mathrm{mg})$ and $5 \mathrm{~mol} \%$ catalyst II $(0.05 \mathrm{mmol}, 3.0 \mathrm{mg})$ in $1.0 \mathrm{~mL} \alpha, \alpha, \alpha$-trifluoromethanebenzene was stirred for $6 \mathrm{~h}$ at room temperature. The reaction mixture was directly purified by silica gel chromatography, eluted by hexane/EtOAc $=3: 1$ to afford the desired product as a white solid $(46.7 \mathrm{mg}$, 99\% yield), $96 \%$ ee (HPLC Daicel CHIRALCEL AS-H column, hexane/ $i \mathrm{PrOH}=70: 30$ at $0.5 \mathrm{~mL} / \mathrm{min}, \lambda$ $=254 \mathrm{~nm}) ; \mathrm{t}_{\text {major }}=23.09 \mathrm{~min}, \mathrm{t}_{\text {minor }}=31.83 \mathrm{~min} ;[\alpha]_{\mathrm{D}}^{22.0}=-11.0(\mathrm{c}=1.00, \mathrm{MeOH})$.

General Procedure for atropo-enantioselective transesterification of biaryl lactones with phenols (Scheme 3, 3u): A mixture of biaryl lactones $1 \mathbf{a}(0.1 \mathrm{mmol}, 31.9 \mathrm{mg})$, phenol $\mathbf{2 u}(0.12 \mathrm{mmol}, 11.3 \mathrm{mg})$ and $5 \mathrm{~mol} \%$ catalyst II $(0.05 \mathrm{mmol}, 3.0 \mathrm{mg})$ in $1.0 \mathrm{~mL} \alpha, \alpha, \alpha$-trifluoromethanebenzene was stirred for $1 \mathrm{~h}$ at $-10{ }^{\circ} \mathrm{C}$. The reaction mixture was directly purified by silica gel chromatography, eluted by dichloromethane to afford the desired product as a white solid (31 mg, 75\% yield), 96\% ee (HPLC Daicel CHIRALCEL AD column, hexane $/ \mathrm{PrOH}=75: 25$ at $0.5 \mathrm{~mL} / \mathrm{min}, \lambda=210 \mathrm{~nm}$ ): $\mathrm{t}_{\text {major }}=15.93 \mathrm{~min}$, $\mathrm{t}_{\mathrm{minor}}=26.88 \mathrm{~min}, ;[\alpha]_{\mathrm{D}}{ }^{25.0}=+7.9\left(\mathrm{c}=1.00, \mathrm{CHCl}_{3}\right)$.

General Procedure for gram scale synthesis (Scheme 5, Eq. 1): A mixture of biaryl lactones 1a (2.5 $\mathrm{mmol}, 798 \mathrm{mg})$, 4-nitrobenzyl alcohol $\mathbf{2 a}(3.0 \mathrm{mmol}, 459 \mathrm{mg})$ and $0.02 \mathrm{~mol} \%$ catalyst II $(0.05 \mathrm{mmol}, 30$ $\mathrm{mg}$ ) in $2.5 \mathrm{~mL} \alpha, \alpha, \alpha$-trifluoromethanebenzene was stirred for $24 \mathrm{~h}$ at room temperature. The reaction mixture was directly purified by silica gel chromatography, eluted by hexane/EtOAc $=3: 1$ to afford the desired product as a white solid (1.167g, 99\% yield), 95\% ee (HPLC Daicel CHIRALCEL AS-H column, hexane $/ \mathrm{PrOH}=70: 30$ at $0.5 \mathrm{~mL} / \mathrm{min}, \lambda=254 \mathrm{~nm}$ ); $\mathrm{t}_{\text {major }}=23.09 \mathrm{~min}, \mathrm{t}_{\mathrm{minor}}=31.83 \mathrm{~min}$.<smiles>COc1ccc(Br)c(-c2c(C)cccc2C(=O)OCc2ccc([N+](=O)[O-])cc2)c1O</smiles>

3a

4-Nitrobenzyl $(R)$-6'-bromo-2'-hydroxy-3'-methoxy-6-methyl-[1,1'-iphenyl]-2-carboxylate $(3 a)$ : White solid; 99\% yield, 96\% ee; $1 \mathrm{H}-\mathrm{NMR}\left(300 \mathrm{MHz}, \mathrm{CDCl}_{3}\right): \delta=8.15(\mathrm{~d}, 2 \mathrm{H}, J=8.7 \mathrm{~Hz}), 7.96(\mathrm{~d}, 1 \mathrm{H}$, $J=7.5 \mathrm{~Hz}), 7.52(\mathrm{~d}, 1 \mathrm{H}, J=7.2 \mathrm{~Hz}), 7.42(\mathrm{t}, 1 \mathrm{H}, J=7.5 \mathrm{~Hz}), 7.34(\mathrm{~d}, 2 \mathrm{H}, J=8.4 \mathrm{~Hz}), 7.07(\mathrm{~d}, 1 \mathrm{H}, J=$ $8.4 \mathrm{~Hz}), 6.64(\mathrm{~d}, 1 \mathrm{H}, J=9.3 \mathrm{~Hz}), 5.58(\mathrm{~s}, 1 \mathrm{H}), 5.19(\mathrm{~d}, 2 \mathrm{H}, J=5.1 \mathrm{~Hz}), 3.85(\mathrm{~s}, 3 \mathrm{H}), 2.06(\mathrm{~s}, 3 \mathrm{H}) .{ }^{13} \mathrm{C}$ NMR (75 MHz, $\mathrm{CDCl}_{3}$, TMS): 166.4, 147.3, 145.5, 143.1, 143.0, 138.1, 1369, 134.2, 129.8, 128.3, 128.1, 128.0, 127.4, 123.3, 122.8, 1145, 110.3, 65.0, 55.8, 19.7. HPLC (Daicel CHIRALCEL AS-H column, hexane $/ \mathrm{PrOH}=70: 30$ at $0.5 \mathrm{~mL} / \mathrm{min}, \lambda=254 \mathrm{~nm}): \mathrm{t}_{\text {major }}=23.09 \mathrm{~min}, \mathrm{t}_{\text {minor }}=31.83 \mathrm{~min}$, ee $=$ $96 \% ;[\alpha]_{\mathrm{D}}^{22.0}($ major $)=-11.0(\mathrm{c}=1.00, \mathrm{MeOH})$. 
<smiles>COc1ccc(Br)c(-c2c(C)cccc2C(=O)OCc2ccccc2[N+](=O)[O-])c1O</smiles>

3b

2-Nitrobenzyl $(R)$-6'-bromo-2'-hydroxy-3'-methoxy-6-methyl-[1,1'-biphenyl]-2-carboxylate (3b): White solid; 98\% yield, 96\% ee; ${ }^{1} \mathrm{H}-\mathrm{NMR}\left(300 \mathrm{MHz}, \mathrm{CDCl}_{3}\right): \delta=8.09\left(\mathrm{dd}, 1 \mathrm{H}, J_{1}=1.2 \mathrm{~Hz}, J_{2}=7.2\right.$ $\mathrm{Hz}), 7.41-7.62(\mathrm{~m}, 5 \mathrm{H}), 7.07(\mathrm{~d}, 1 \mathrm{H}, J=8.7 \mathrm{~Hz}), 6.67(\mathrm{~d}, 1 \mathrm{H}, J=8.7 \mathrm{~Hz}), 5.64(\mathrm{~s}, 1 \mathrm{H}), 5.55(\mathrm{~d}, 2 \mathrm{H}, J=$ $2.1 \mathrm{~Hz}), 3.87(\mathrm{~s}, 3 \mathrm{H}), 2.08(\mathrm{~s}, 3 \mathrm{H}) .{ }^{13} \mathrm{C}$ NMR $\left(75 \mathrm{MHz}, \mathrm{CDCl}_{3}, \mathrm{TMS}\right): \delta=166.1,147.2,145.6,143.3$, 138.3, 137.3, 134.3, 133.6, 132.4, 129.8, 129.0, 128.4, 128.3, 128.1, 127.5, 124.9, 122.9, 114.6, 110.5, 63.2, 55.9, 19.8; HPLC (Daicel CHIRALCEL AD column, hexane $/ \mathrm{PrOH}=65: 35$ at $0.5 \mathrm{~mL} / \mathrm{min}, \lambda=$ $254 \mathrm{~nm}): \mathrm{t}_{\text {major }}=13.66 \mathrm{~min}, \mathrm{t}_{\text {minor }}=24.24 \mathrm{~min}, \mathrm{ee}=96 \% ;[\alpha]_{\mathrm{D}}^{22.3}($ major $)=-7.0(\mathrm{c}=1.00, \mathrm{MeOH})$.<smiles>COc1ccc(COC(=O)c2cccc(C)c2-c2c(Br)ccc(OC)c2O)cc1</smiles>

3c

4-Methoxybenzyl $\quad(R)$-6'-bromo-2'-hydroxy-3'-methoxy-6-methyl-[1,1'-biphenyl]-2-carboxylate (3c): White solid; quantitative yield, $93 \%$ ee; ${ }^{1} \mathrm{H}-\mathrm{NMR}\left(300 \mathrm{MHz}, \mathrm{CDCl}_{3}\right): \delta=7.92(\mathrm{~d}, 1 \mathrm{H}, J=7.2 \mathrm{~Hz})$, $7.48(\mathrm{~d}, 1 \mathrm{H}, J=6.9 \mathrm{~Hz}), 7.37(\mathrm{t}, 1 \mathrm{H}, J=7.5 \mathrm{~Hz}), 7.15(\mathrm{~d}, 1 \mathrm{H}, J=8.4 \mathrm{~Hz}), 7.07(\mathrm{~d}, 1 \mathrm{H}, J=8.7 \mathrm{~Hz}), 6.83$ $(\mathrm{d}, 2 \mathrm{H}, J=6.9 \mathrm{~Hz}), 6.65(\mathrm{~d}, 1 \mathrm{H}, J=8.7 \mathrm{~Hz}), 5.54(\mathrm{~s}, 1 \mathrm{H}), 4.98-5.07(\mathrm{~m}, 2 \mathrm{H}), 3.86(\mathrm{~s}, 3 \mathrm{H}), 3.81(\mathrm{~s}, 3 \mathrm{H})$, 2.06 (s, 3H). ${ }^{13} \mathrm{C}$ NMR $\left(75 \mathrm{MHz}, \mathrm{CDCl}_{3}\right.$, TMS): $\delta=166.8,159.4,145.6,143.2,138.0,136.9,133.8$, 130.6, 128.2, 128.0, 127.7, 122.9, 114.7, 113.7, 110.4, 66.4, 55.9, 55.3, 19.8; HPLC (Daicel CHIRALCEL AS-H column, hexane $/ \mathrm{iPrOH}=65: 35$ at $0.5 \mathrm{~mL} / \mathrm{min}, \lambda=254 \mathrm{~nm}): \mathrm{t}_{\text {major }}=13.75 \mathrm{~min}$, $\mathrm{t}_{\text {minor }}=30.60 \mathrm{~min}, \mathrm{ee}=93 \% ;[\alpha]_{\mathrm{D}}^{25.2}$ (major) $=+15.7\left(\mathrm{c}=1.00, \mathrm{CHCl}_{3}\right)$.<smiles>COc1ccc(Br)c(-c2c(C)cccc2C(=O)OCc2ccc3c(c2)OCO3)c1O</smiles>

3d

Benzo[d][1,3]dioxol-5-ylmethyl $(R)-6$ '-bromo-2'-hydroxy-3'-methoxy-6-methyl-[1,1'-biphenyl]-2carboxylate (3d): White solid; 93\% yield, $91 \%$ ee; ${ }^{1} \mathrm{H}-\mathrm{NMR}\left(300 \mathrm{MHz}, \mathrm{CDCl}_{3}\right): \delta 7.92(\mathrm{~d}, 1 \mathrm{H}, J=7.5$ $\mathrm{Hz}), 7.48(\mathrm{~d}, 1 \mathrm{H}, J=7.5 \mathrm{~Hz}), 7.38(\mathrm{t}, 1 \mathrm{H}, J=7.8 \mathrm{~Hz}), 7.08(\mathrm{~d}, 1 \mathrm{H}, J=8.7 \mathrm{~Hz}), 6.65-6.71(\mathrm{~m}, 4 \mathrm{H}), 5.96$ (s, 2H), $5.56(\mathrm{~s}, 1 \mathrm{H}), 4.98-4.99(\mathrm{~m}, 2 \mathrm{H}), 3.88(\mathrm{~s}, 3 \mathrm{H}), 2.05$ (s, 3H). ${ }^{13} \mathrm{C} \mathrm{NMR}\left(75 \mathrm{MHz}, \mathrm{CDCl}_{3}, \mathrm{TMS}\right): \delta$ $=166.8,147.5,147.4,145.6,143.3,138.1,136.9,133.9,130.6,129.5,128.2,128.0,122.9,122.3,120.5$, 
114.8, 110.5, 109.1, 107.9, 101.0, 66.6, 55.9, 19.8; HPLC (Daicel CHIRALCEL AS-H column, hexane $/ \mathrm{iPrOH}=75: 25$ at $0.5 \mathrm{~mL} / \mathrm{min}, \lambda=254 \mathrm{~nm}): \mathrm{t}_{\text {major }}=21.84 \mathrm{~min}, \mathrm{t}_{\text {minor }}=35.22 \mathrm{~min}$, ee $=91 \%$; $[\alpha]_{\mathrm{D}}^{25.2}$ (major) $=+5.1\left(\mathrm{c}=1.00, \mathrm{CDCl}_{3}\right)$.<smiles>COc1ccc(Br)c(-c2c(C)cccc2C(=O)OCc2c3ccccc3cc3ccccc23)c1O</smiles>

Anthracen-9-ylmethyl

(R)-6'-bromo-2'-hydroxy-3'-methoxy-6-methyl-[1,1'-biphenyl]-2carboxylate (3e): White solid; 98\% yield, 90\% ee; ${ }^{1} \mathrm{H}-\mathrm{NMR}\left(300 \mathrm{MHz}, \mathrm{CDCl}_{3}\right): \delta=8.47(\mathrm{~s}, 1 \mathrm{H}), 8.13$ $8.16(\mathrm{~m}, 2 \mathrm{H}), 8.00-8.04(\mathrm{~m}, 3 \mathrm{H}), 7.35-7.53(\mathrm{~m}, 6 \mathrm{H}), 6.07-6.12(\mathrm{~m}, 3 \mathrm{H}), 5.65(\mathrm{~d}, 1 \mathrm{H}, J=8.7 \mathrm{~Hz}), 5.13(\mathrm{~s}$, $1 \mathrm{H}), 3.34(\mathrm{~s}, 3 \mathrm{H}), 1.92(\mathrm{~s}, 3 \mathrm{H}) .{ }^{13} \mathrm{C}$ NMR $\left(75 \mathrm{MHz}, \mathrm{CDCl}_{3}\right.$, TMS): $\delta=167.5,144.6,142.5,138.0,137.1$, 134.0, 131.3, 131.1, 128.8, 128.6, 128.3, 128.0, 127.2, 126.3, 126.0, 125.1, 124.2, 122.1, 114.0, 109.0, 58.9, 55.2, 19.8; HPLC (Daicel CHIRALCEL AD column, hexane $/ \mathrm{PrOH}=70: 30$ at $0.5 \mathrm{~mL} / \mathrm{min}, \lambda=$ $254 \mathrm{~nm}): \mathrm{t}_{\text {major }}=13.16 \mathrm{~min}, \mathrm{t}_{\text {minor }}=22.26 \mathrm{~min}$, ee $=90 \% ;[\alpha]_{\mathrm{D}}^{23.0}($ major $)=-6.4\left(\mathrm{c}=1.00, \mathrm{CHCl}_{3}\right)$.<smiles>COc1ccc(Br)c(-c2c(C)cccc2C(=O)OC2c3ccccc3-c3ccccc32)c1O</smiles>

$3 f$

9H-Fluoren-9-yl (R)-6'-bromo-2'-hydroxy-3'-methoxy-6-methyl-[1,1'-biphenyl]-2-carboxylate (3f): White solid; 97\% yield, 92\% ee; ${ }^{1} \mathrm{H}-\mathrm{NMR}\left(300 \mathrm{MHz}, \mathrm{CDCl}_{3}\right): \delta=7.98(\mathrm{~d}, 1 \mathrm{H}, J=7.5 \mathrm{~Hz}), 7.61(\mathrm{~d}, 2 \mathrm{H}$, $J=7.5 \mathrm{~Hz}), 7.43-7.51(\mathrm{~m}, 3 \mathrm{H}), 7.34-7.41(\mathrm{~m}, 3 \mathrm{H}), 7.20-7.25(\mathrm{~m}, 2 \mathrm{H}), 6.83-6.86(\mathrm{~m}, 2 \mathrm{H}), 6.46(\mathrm{~d}, 1 \mathrm{H}, J$ $=8.4 \mathrm{~Hz}), 5.45(\mathrm{~s}, 1 \mathrm{H}), 3.73(\mathrm{~s}, 3 \mathrm{H}), 2.07(\mathrm{~s}, 3 \mathrm{H}) .{ }^{13} \mathrm{C} \mathrm{NMR}\left(75 \mathrm{MHz}, \mathrm{CDCl}_{3}, \mathrm{TMS}\right): \delta=167.6,145.4$, 143.2, 142.1, 141.9, 140.9, 140.8, 138.1, 137.3, 134.0, 130.5, 129.1, 129.0, 128.3, 128.0, 127.6, 126.1, 126.0, 122.7, 119.5, 114.5, 110.2, 75.0, 55.8, 19.8; HPLC (Daicel CHIRALCEL AS-H column, hexane $/ \mathrm{iPrOH}=90: 10$ at $0.5 \mathrm{~mL} / \mathrm{min}, \lambda=254 \mathrm{~nm}): \mathrm{t}_{\text {major }}=17.66 \mathrm{~min}, \mathrm{t}_{\text {minor }}=23.68 \mathrm{~min}$, ee $=92 \%$; $[\alpha]_{\mathrm{D}}^{23.4}$ (major) $=+7.2\left(\mathrm{c}=1.01, \mathrm{CHCl}_{3}\right)$.<smiles>COc1ccc(Br)c(-c2c(C)cccc2C(=O)OCc2ccco2)c1O</smiles>

$3 g$ 
Furan-2-ylmethyl (R)-6'-bromo-2'-hydroxy-3'-methoxy-6-methyl-[1,1'-biphenyl]-2- carboxylate (3g): White solid; 96\% yield, 90\% ee; ${ }^{1} \mathrm{H}-\mathrm{NMR}\left(300 \mathrm{MHz}, \mathrm{CDCl}_{3}\right): \delta=7.92(\mathrm{~d}, 1 \mathrm{H}, J=7.8 \mathrm{~Hz}), 7.49$ $(\mathrm{d}, 1 \mathrm{H}, J=7.5 \mathrm{~Hz}), 7.35-7.40(\mathrm{~m}, 2 \mathrm{H}), 7.11(\mathrm{~d}, 1 \mathrm{H}, J=8.7 \mathrm{~Hz}), 6.72(\mathrm{~d}, 1 \mathrm{H}, J=8.7 \mathrm{~Hz}), 6.29-6.33(\mathrm{~m}$, 2H), $5.57(\mathrm{~s}, 1 \mathrm{H}), 5.05(\mathrm{~s}, 2 \mathrm{H}), 3.90(\mathrm{~s}, 3 \mathrm{H}), 2.07(\mathrm{~s}, 3 \mathrm{H}) .{ }^{13} \mathrm{C}$ NMR $\left(75 \mathrm{MHz}, \mathrm{CDCl}_{3}, \mathrm{TMS}\right): \delta=166.3$, 149.4, 145.6, 143.2, 138.1, 137.2, 134.0, 130.1, 128.3, 128.0, 127.5, 122.9, 114.7, 110.5, 110.4, 110.3, 58.4, 55.9, 19.8; HPLC (Daicel CHIRALCEL AD-H column, hexane $/ \mathrm{PPOH}=65: 35$ at $0.5 \mathrm{~mL} / \mathrm{min}, \lambda=$ $254 \mathrm{~nm}): \mathrm{t}_{\text {major }}=11.28 \mathrm{~min}, \mathrm{t}_{\text {minor }}=23.98 \mathrm{~min}$, ee $=90 \% ;[\alpha]_{\mathrm{D}}{ }^{26.1}($ major $)=+12.2\left(\mathrm{c}=1.00, \mathrm{CHCl}_{3}\right)$.

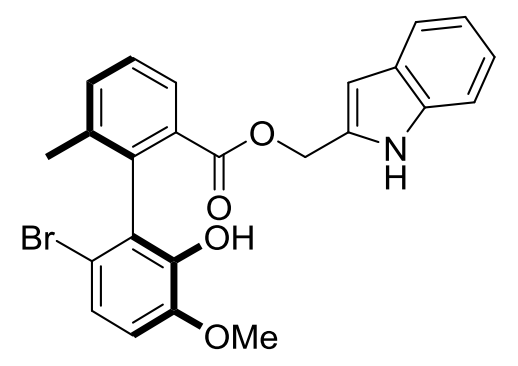

3h

(1H-Indol-2-yl)methyl

(R)-6'-bromo-2'-hydroxy-3'-methoxy-6-methyl-[1,1'-biphenyl]-2carboxylate (3h): White solid; quantitative yield, $91 \%$ ee; ${ }^{1} \mathrm{H}-\mathrm{NMR}\left(300 \mathrm{MHz}, \mathrm{CDCl}_{3}\right): \delta=8.25(\mathrm{~s}$, $1 \mathrm{H}), 7.89(\mathrm{~d}, 1 \mathrm{H}, J=7.2 \mathrm{~Hz}), 7.56(\mathrm{~d}, 1 \mathrm{H}, J=7.8 \mathrm{~Hz}), 7.49(\mathrm{~d}, 1 \mathrm{H}, J=7.2 \mathrm{~Hz}), 7.39(\mathrm{t}, 1 \mathrm{H}, J=7.5 \mathrm{~Hz})$, $7.29(\mathrm{~d}, 1 \mathrm{H}, J=8.4 \mathrm{~Hz}), 7.19\left(\mathrm{dt}, 1 \mathrm{H}, J_{l}=1.2 \mathrm{~Hz}, J_{2}=7.8 \mathrm{~Hz}\right), 7.09\left(\mathrm{dt}, 1 \mathrm{H}, J_{I}=1.2 \mathrm{~Hz}, J_{2}=7.8 \mathrm{~Hz}\right)$, $7.04(\mathrm{~d}, 1 \mathrm{H}, J=8.4 \mathrm{~Hz}), 6.55(\mathrm{~d}, 1 \mathrm{H}, J=8.7 \mathrm{~Hz}), 6.39(\mathrm{~d}, 1 \mathrm{H}, J=1.2 \mathrm{~Hz}), 5.55(\mathrm{~s}, 1 \mathrm{H}), 5.17-5.27(\mathrm{~m}$, $2 \mathrm{H}), 3.68(\mathrm{~s}, 3 \mathrm{H}), 2.06(\mathrm{~s}, 3 \mathrm{H}) .{ }^{13} \mathrm{C}$ NMR $\left(75 \mathrm{MHz}, \mathrm{CDCl}_{3}, \mathrm{TMS}\right): \delta=168.1,145.6,143.3,138.1,136.7$, 136.6, 134.1, 132.8, 130.5, 128.1, 128.0, 127.6, 122.8, 122.4, 120.7, 119.7, 114.6, 111.0, 110.5, 103.2, 60.0, 55.8, 19.8; HPLC (Daicel CHIRALCEL AS-H column, hexane $/ \mathrm{PrOH}=90: 10$ at $0.5 \mathrm{~mL} / \mathrm{min}, \lambda=$ $254 \mathrm{~nm}): \mathrm{t}_{\text {major }}=50.74 \mathrm{~min}, \mathrm{t}_{\text {minor }}=39.31 \mathrm{~min}, \mathrm{ee}=90 \% ;[\alpha]_{\mathrm{D}}^{25.6}($ major $)=+37.4\left(\mathrm{c}=1.00, \mathrm{CHCl}_{3}\right)$.<smiles>COc1ccc(Br)c(-c2c(C)cccc2C(=O)OCc2cccnc2)c1O</smiles>

$3 \mathbf{i}$

Pyridin-3-ylmethyl $\quad(R)$-6'-bromo-2'-hydroxy-3'-methoxy-6-methyl-[1,1'-biphenyl]-2-carboxylate (3i): White solid; quantitative yield, $90 \%$ ee; ${ }^{1} \mathrm{H}-\mathrm{NMR}\left(300 \mathrm{MHz}, \mathrm{CDCl}_{3}\right): \delta=8.54(\mathrm{~s}, 1 \mathrm{H}), 8.40(\mathrm{~s}$, $1 \mathrm{H}), 7.93(\mathrm{~d}, 1 \mathrm{H}, J=7.5 \mathrm{~Hz}), 7.48-7.56(\mathrm{~m}, 2 \mathrm{H}), 7.39(\mathrm{~d}, 1 \mathrm{H}, J=7.5 \mathrm{~Hz}), 7.21-7.24(\mathrm{~m}, 1 \mathrm{H}), 7.02(\mathrm{~d}$, $1 \mathrm{H}, J=8.7 \mathrm{~Hz}), 6.62(\mathrm{~d}, 1 \mathrm{H}, J=8.7 \mathrm{~Hz}), 5.08(\mathrm{~s}, 2 \mathrm{H}), 3.85(\mathrm{~s}, 3 \mathrm{H}), 2.05(\mathrm{~s}, 3 \mathrm{H}) .{ }^{13} \mathrm{C}$ NMR $(75 \mathrm{MHz}$, $\left.\mathrm{CDCl}_{3}, \mathrm{TMS}\right): \delta=166.7,149.5,149.1,145.6,143.3,138.2,137.0,136.3,134.1,130.1,128.3,128.0$, 127.6, 123.3, 122.8, 114.6, 110.5, 64.0, 55.9, 19.8; HPLC (Daicel CHIRALCEL AD column, hexane $/ \mathrm{PrOH}=60: 40$ at $0.5 \mathrm{~mL} / \mathrm{min}, \lambda=254 \mathrm{~nm}): \mathrm{t}_{\text {major }}=11.98 \mathrm{~min}, \mathrm{t}_{\text {minor }}=23.77 \mathrm{~min}$, ee $=90 \%$; $[\alpha]_{\mathrm{D}}^{23.5}$ (major) $=+11.9\left(\mathrm{c}=1.00, \mathrm{CHCl}_{3}\right)$.<smiles>COc1ccc(Br)c(-c2c(C)cccc2C(=O)OCc2ccnc3ccccc23)c1O</smiles> 
Quinolin-4-ylmethyl (R)-6'-bromo-2'-hydroxy-3'-methoxy-6-methyl-[1,1'-biphenyl]-2-carboxylate (3j): White solid; 97\% yield, 93\% ee; ${ }^{1} \mathrm{H}-\mathrm{NMR}\left(300 \mathrm{MHz}, \mathrm{CDCl}_{3}\right): \delta=8.82(\mathrm{~d}, 1 \mathrm{H}, J=4.2 \mathrm{~Hz}), 8.14$ $(\mathrm{d}, 1 \mathrm{H}, J=8.4 \mathrm{~Hz}), 8.02(\mathrm{~d}, 1 \mathrm{H}, J=7.8 \mathrm{~Hz}), 7.84(\mathrm{~d}, 1 \mathrm{H}, J=8.4 \mathrm{~Hz}), 7.73(\mathrm{t}, 1 \mathrm{H}, J=7.8 \mathrm{~Hz}), 7.50-7.58$ $(\mathrm{m}, 2 \mathrm{H}), 7.41(\mathrm{t}, 1 \mathrm{H}, J=7.8 \mathrm{~Hz}), 7.26-7.27(\mathrm{~m}, 1 \mathrm{H}), 6.78(\mathrm{~d}, 1 \mathrm{H}, J=8.7 \mathrm{~Hz}), 6.33(\mathrm{~d}, 1 \mathrm{H}, J=8.7 \mathrm{~Hz})$, $5.66(\mathrm{~s}, 1 \mathrm{H}), 5.50-5.60(\mathrm{~m}, 2 \mathrm{H}), 3.70(\mathrm{~s}, 3 \mathrm{H}), 2.03(\mathrm{~s}, 3 \mathrm{H}) .{ }^{13} \mathrm{C} \mathrm{NMR}\left(75 \mathrm{MHz}, \mathrm{CDCl}_{3}, \mathrm{TMS}\right): \delta=166.6$, 149.9, 147.9, 145.3, 143.0, 140.9, 138.3, 137.2, 134.3, 129.9, 129.4, 1285, 128.1, 127.5, 127.0, 126.2, 120.3, 114.4, 110.0, 62.9, 55.7, 19.9; HPLC (Daicel CHIRALCEL AD column, hexane $/ \mathrm{PrOH}=60: 40$ at $0.5 \mathrm{~mL} / \mathrm{min}, \lambda=254 \mathrm{~nm}): \mathrm{t}_{\text {major }}=16.27 \mathrm{~min}, \mathrm{t}_{\text {minor }}=35.66 \mathrm{~min}$, ee $=93 \%$; $[\alpha]_{\mathrm{D}}{ }^{23.6}($ major $)=-3.4(\mathrm{c}=$ $\left.1.00, \mathrm{CHCl}_{3}\right)$.

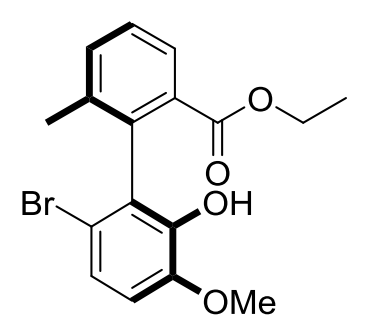

3k

Ethyl (R)-6'-bromo-2'-hydroxy-3'-methoxy-6-methyl-[1,1'-biphenyl]-2-carboxylate (3k): White solid; $97 \%$ yield, $92 \%$ ee; ${ }^{1} \mathrm{H}-\mathrm{NMR}\left(300 \mathrm{MHz}, \mathrm{CDCl}_{3}\right): \delta=7.91(\mathrm{~d}, 1 \mathrm{H}, J=7.8 \mathrm{~Hz}), 7.49(\mathrm{~d}, 1 \mathrm{H}, J=$ $7.5 \mathrm{~Hz}), 7.39(\mathrm{t}, 1 \mathrm{H}, J=7.8 \mathrm{~Hz}), 7.16(\mathrm{~d}, 1 \mathrm{H}, J=8.7 \mathrm{~Hz}), 6.78(\mathrm{~d}, 1 \mathrm{H}, J=8.7 \mathrm{~Hz}), 4.10(\mathrm{q}, 2 \mathrm{H}, J=6.9$ $\mathrm{Hz}), 3.92(\mathrm{~s}, 3 \mathrm{H}), 2.08(\mathrm{~s}, 3 \mathrm{H}), 1.07(\mathrm{t}, 3 \mathrm{H}, J=6.9 \mathrm{~Hz}) .{ }^{13} \mathrm{C} \mathrm{NMR}\left(75 \mathrm{MHz}, \mathrm{CDCl}_{3}, \mathrm{TMS}\right): \delta=166.9$, 145.7, 143.4, 138.0, 136.8, 133.7, 130.9, 128.0, 122.9, 114.9, 110.6, 60.6, 56.1, 19.8, 13.7; HPLC (Daicel CHIRALCEL AD column, hexane $/ \mathrm{PrOH}=65: 35$ at $0.5 \mathrm{~mL} / \mathrm{min}, \lambda=254 \mathrm{~nm}$ ): $\mathrm{t}_{\text {major }}=9.72 \mathrm{~min}$, $\mathrm{t}_{\text {minor }}=15.47 \mathrm{~min}$, ee $=92 \% ;[\alpha]_{\mathrm{D}}^{25.3}($ major $)=+4.8\left(\mathrm{c}=1.00, \mathrm{CHCl}_{3}\right)$.

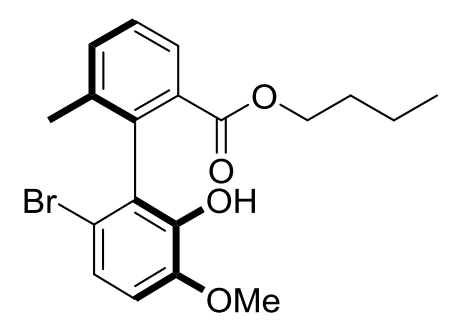

31

Butyl (R)-6'-bromo-2'-hydroxy-3'-methoxy-6-methyl-[1,1'-biphenyl]-2-carboxylate (3l): White solid; $90 \%$ yield, $90 \%$ ee; ${ }^{1} \mathrm{H}-\mathrm{NMR}\left(300 \mathrm{MHz}, \mathrm{CDCl}_{3}\right): \delta=7.92\left(\mathrm{dd}, 1 \mathrm{H}, J_{1}=0.9 \mathrm{~Hz}, J_{2}=7.8 \mathrm{~Hz}\right)$, $7.49(\mathrm{~d}, 1 \mathrm{H}, J=6.9 \mathrm{~Hz}), 7.39(\mathrm{t}, 1 \mathrm{H}, J=7.5 \mathrm{~Hz}), 7.16(\mathrm{~d}, 1 \mathrm{H}, J=8.7 \mathrm{~Hz}), 6.77(\mathrm{~d}, 1 \mathrm{H}, J=8.7 \mathrm{~Hz}), 5.60$ $(\mathrm{s}, 1 \mathrm{H}), 4.05(\mathrm{t}, 2 \mathrm{H}, J=6.3 \mathrm{~Hz}), 3.92(\mathrm{~s}, 3 \mathrm{H}), 2.07(\mathrm{~s}, 3 \mathrm{H}), 1.32-1.46(\mathrm{~m}, 2 \mathrm{H}), 1.22-1.29(\mathrm{~m}, 2 \mathrm{H}), 1.22-$ $1.30(\mathrm{~m}, 2 \mathrm{H}), 0.86(\mathrm{t}, 3 \mathrm{H}, J=7.2 \mathrm{~Hz}) .{ }^{13} \mathrm{C} \mathrm{NMR}\left(75 \mathrm{MHz}, \mathrm{CDCl}_{3}, \mathrm{TMS}\right): \delta=167.1,145.7,143.3,138.0$, 136.8, 133.7, 130.9, 128.1, 128.0, 127.9, 122.9, 114.9, 110.5, 64.7, 56.0, 30.4, 19.9, 19.1, 13.7; HPLC (Daicel CHIRALCEL AD column, hexane $/ \mathrm{PrOH}=65: 35$ at $0.5 \mathrm{~mL} / \mathrm{min}, \lambda=254 \mathrm{~nm}$ ): $\mathrm{t}_{\text {major }}=8.93 \mathrm{~min}$, $\mathrm{t}_{\text {minor }}=14.42 \mathrm{~min}$, ee $=90 \% ;[\alpha]_{\mathrm{D}}^{25.3}($ major $)=+4.0\left(\mathrm{c}=1.00, \mathrm{CHCl}_{3}\right)$. 


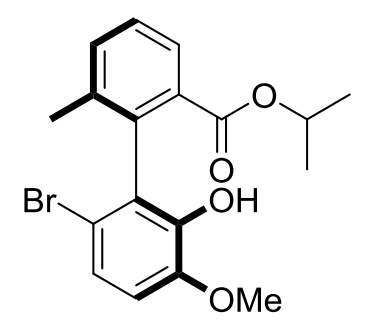

$3 \mathrm{~m}$

Isopropyl $(R)-6$ '-bromo-2'-hydroxy-3'-methoxy-6-methyl-[1,1'-biphenyl]-2-carboxylate (3m): White solid; $90 \%$ yield, 94\% ee; ${ }^{1} \mathrm{H}-\mathrm{NMR}\left(300 \mathrm{MHz}, \mathrm{CDCl}_{3}\right): \delta=7.89(\mathrm{~d}, 1 \mathrm{H}, J=7.5 \mathrm{~Hz}), 7.47(\mathrm{~d}, 1 \mathrm{H}$, $J=6.9 \mathrm{~Hz}), 7.39(\mathrm{t}, 1 \mathrm{H}, J=7.5 \mathrm{~Hz}), 7.16(\mathrm{~d}, 1 \mathrm{H}, J=8.7 \mathrm{~Hz}), 6.78(\mathrm{~d}, 1 \mathrm{H}, J=8.7 \mathrm{~Hz}), 5.60(\mathrm{~s}, 1 \mathrm{H}), 4.98$ (septet, $2 \mathrm{H}, J=6.3 \mathrm{~Hz}), 3.92(\mathrm{~s}, 3 \mathrm{H}), 2.07(\mathrm{~s}, 3 \mathrm{H}), 1.01-1.05(\mathrm{~m}, 6 \mathrm{H}) .{ }^{13} \mathrm{C} \mathrm{NMR}\left(75 \mathrm{MHz}, \mathrm{CDCl}_{3}\right.$, TMS): $\delta=166.7,145.8,143.5,137.9,136.5,133.5,131.6,138.1,128.0,122.9,115.1,110.6,67.8,56.1$, 21.4, 21.3, 19.9; HPLC (Daicel CHIRALCEL AS-H column, hexane $/ \mathrm{PrOH}=90: 10$ at $0.5 \mathrm{~mL} / \mathrm{min}, \lambda=$ $254 \mathrm{~nm}): \mathrm{t}_{\text {major }}=14.09 \mathrm{~min}, \mathrm{t}_{\mathrm{minor}}=18.18 \mathrm{~min}, \mathrm{ee}=94 \% ;[\alpha]_{\mathrm{D}}^{23.9}$ (major) $=-3.2\left(\mathrm{c}=1.00, \mathrm{CHCl}_{3}\right)$.<smiles>COc1ccc(Br)c(-c2c(C)cccc2C(=O)OCC(F)(F)F)c1O</smiles>

3n

2,2,2-Trifluoroethyl $(R)-6$ '-bromo-2'-hydroxy-3'-methoxy-6-methyl-[1,1'-biphenyl]-2-carboxylate (3n): White solid; quantitative yield, 93\% ee; ${ }^{1} \mathrm{H}-\mathrm{NMR}\left(300 \mathrm{MHz}, \mathrm{CDCl}_{3}\right): \delta=7.98(\mathrm{~d}, 1 \mathrm{H}, J=7.8 \mathrm{~Hz})$, $7.55(\mathrm{~d}, 1 \mathrm{H}, J=7.5 \mathrm{~Hz}), 7.43(\mathrm{t}, 1 \mathrm{H}, J=7.8 \mathrm{~Hz}), 7.18(\mathrm{~d}, 1 \mathrm{H}, J=8.4 \mathrm{~Hz}), 6.79(\mathrm{~d}, 1 \mathrm{H}, J=8.7 \mathrm{~Hz}), 4.46$ $\left(\mathrm{dq}, 2 \mathrm{H}, J_{1}=2.1 \mathrm{~Hz}, J_{2}=8.4 \mathrm{~Hz}\right), 3.92(\mathrm{~s}, 3 \mathrm{H}), 2.09(\mathrm{~s}, 3 \mathrm{H}) .{ }^{13} \mathrm{C} \mathrm{NMR}\left(75 \mathrm{MHz}, \mathrm{CDCl}_{3}, \mathrm{TMS}\right): \delta=$ $165.0,145.8,143.3,138.5,137.8,134.8,128.6,128.4,128.1,127.1,123.1,122.9$ (q, $J_{\mathrm{C}, \mathrm{F}}=275.6 \mathrm{~Hz}$, $\mathrm{CF}_{3}$ ), 114.5, 110.8, $60.7\left(\mathrm{q}, J_{\mathrm{C}, \mathrm{F}}=36.4 \mathrm{~Hz}, \mathrm{CH}_{2}\right) ;{ }^{19} \mathrm{~F}\left(\mathrm{CDCl}_{3}\right) \delta=72.1$. HPLC (Daicel CHIRALCEL AD column, hexane $/ i \operatorname{PrOH}=70: 30$ at $0.5 \mathrm{~mL} / \mathrm{min}, \lambda=254 \mathrm{~nm}): t_{\text {major }}=9.18 \mathrm{~min}, \mathrm{t}_{\text {minor }}=36.45 \mathrm{~min}$, ee $=93 \% ;[\alpha]_{\mathrm{D}}{ }^{24.3}$ (major) $=+4.4\left(\mathrm{c}=1.00, \mathrm{CHCl}_{3}\right) .{ }^{19} \mathrm{~F}\left(282 \mathrm{MHz}, \mathrm{CDCl}_{3}\right): \delta-72.1$.<smiles>COc1ccc(Br)c(-c2c(C)cccc2C(=O)OCC(Cl)(Cl)Cl)c1O</smiles>

30

2,2,2-Trichloroethyl $(R)-6$ '-bromo-2'-hydroxy-3'-methoxy-6-methyl-[1,1'-biphenyl]-2-carboxylate (3o): White solid; quantitative yield, $90 \%$ ee; ${ }^{1} \mathrm{H}-\mathrm{NMR}\left(300 \mathrm{MHz}, \mathrm{CDCl}_{3}\right): \delta=8.06(\mathrm{~d}, 1 \mathrm{H}, J=7.8 \mathrm{~Hz})$, $7.56(\mathrm{~d}, 1 \mathrm{H}, J=7.5 \mathrm{~Hz}), 7.44(\mathrm{t}, 1 \mathrm{H}, J=7.8 \mathrm{~Hz}), 7.18(\mathrm{~d}, 1 \mathrm{H}, J=8.7 \mathrm{~Hz}), 6.78(\mathrm{~d}, 1 \mathrm{H}, J=8.7 \mathrm{~Hz}), 5.65$ $(\mathrm{s}, 1 \mathrm{H}), 4.73-4.83(\mathrm{~m}, 2 \mathrm{H}), 3.92(\mathrm{~s}, 3 \mathrm{H}), 2.09(\mathrm{~s}, 3 \mathrm{H}) \cdot{ }^{13} \mathrm{C} \mathrm{NMR}\left(75 \mathrm{MHz}, \mathrm{CDCl}_{3}, \mathrm{TMS}\right): \delta=164.8$, $145.8,143.4$, 138.5, 137.8, 134.8, 128.6, 128.2, 127.2, 123.1, 114.6, 110.8, 95.0, 74.5, 56.0, 19.8; HPLC 
(Daicel CHIRALCEL AD column, hexane $/ \mathrm{PrOH}=65: 35$ at $0.5 \mathrm{~mL} / \mathrm{min}, \lambda=254 \mathrm{~nm}$ ): $\mathrm{t}_{\text {major }}=9.89 \mathrm{~min}$, $\mathrm{t}_{\text {minor }}=25.49 \min$, ee $=90 \% ;[\alpha]_{\mathrm{D}}^{25.3}($ major $)=+3.1\left(\mathrm{c}=1.01, \mathrm{CHCl}_{3}\right)$.<smiles>COCCOC(=O)c1cccc(C)c1-c1c(Br)ccc(OC)c1O</smiles>

2-Methoxyethyl (R)-6'-bromo-2'-hydroxy-3'-methoxy-6-methyl-[1,1'-biphenyl]-2-carboxylate (3p): White solid; quantitative yield, $95 \%$ ee; ${ }^{1} \mathrm{H}-\mathrm{NMR}\left(300 \mathrm{MHz}, \mathrm{CDCl}_{3}\right): \delta=7.95(\mathrm{~d}, 1 \mathrm{H}, J=7.8 \mathrm{~Hz}), 7.49$ $(\mathrm{d}, 1 \mathrm{H}, J=7.5 \mathrm{~Hz}), 7.39(\mathrm{t}, 1 \mathrm{H}, J=7.8 \mathrm{~Hz}), 7.17(\mathrm{~d}, 1 \mathrm{H}, J=8.7 \mathrm{~Hz}), 6.78(\mathrm{~d}, 1 \mathrm{H}, J=8.7 \mathrm{~Hz}), 5.63(\mathrm{~s}$, $1 \mathrm{H}), 4.22(\mathrm{t}, 2 \mathrm{H}, J=4.8 \mathrm{~Hz}), 3.92(\mathrm{~s}, 3 \mathrm{H}), 6.78(\mathrm{~d}, 1 \mathrm{H}, J=8.7 \mathrm{~Hz}), 3.43-3.47(\mathrm{~m}, 2 \mathrm{H}), 3.33(\mathrm{~s}, 3 \mathrm{H})$, $2.07(\mathrm{~s}, 3 \mathrm{H}) .{ }^{13} \mathrm{C} \mathrm{NMR}\left(75 \mathrm{MHz}, \mathrm{CDCl}_{3}, \mathrm{TMS}\right): \delta=166.6,145.8,143.4,138.0,137.1,133.9,130.3$, 128.2, 128.0, 127.8, 122..9, 114.8, 110.5, 70.2, 63.7, 58.8, 56.0, 19.8; HPLC (Daicel CHIRALCEL AD column, hexane $/ \mathrm{PrOH}=65: 35$ at $0.5 \mathrm{~mL} / \mathrm{min}, \lambda=254 \mathrm{~nm}): \mathrm{t}_{\text {major }}=10.35 \mathrm{~min}, \mathrm{t}_{\text {minor }}=18.35 \mathrm{~min}$, ee $=$ $95 \% ;[\alpha]_{\mathrm{D}}^{25.9}$ (major) $=+4.1\left(\mathrm{c}=1.00, \mathrm{CHCl}_{3}\right)$.<smiles>COc1ccc(Br)c(-c2c(C)cccc2C(=O)OCCOc2ccccc2)c1O</smiles>

$3 q$

2-Phenoxyethyl $(R)-6$ '-bromo-2'-hydroxy-3'-methoxy-6-methyl-[1,1'-biphenyl]-2-carboxylate (3q): White solid; quantitative yield, 90\% ee; ${ }^{1} \mathrm{H}-\mathrm{NMR}\left(300 \mathrm{MHz}, \mathrm{CDCl}_{3}\right): \delta=7.97(\mathrm{~d}, 1 \mathrm{H}, J=6.9 \mathrm{~Hz}), 7.50$ $(\mathrm{d}, 1 \mathrm{H}, J=7.5 \mathrm{~Hz}), 7.40(\mathrm{t}, 1 \mathrm{H}, J=7.5 \mathrm{~Hz}), 7.28-7.33(\mathrm{~m}, 2 \mathrm{H}), 7.09(\mathrm{~d}, 1 \mathrm{H}, J=8.7 \mathrm{~Hz}), 6.96(\mathrm{t}, 1 \mathrm{H}, J$ $=7.2 \mathrm{~Hz}), 6.85(\mathrm{~d}, 2 \mathrm{H}, J=8.1 \mathrm{~Hz}), 6.64(\mathrm{~d}, 2 \mathrm{H}, J=8.7 \mathrm{~Hz}), 5.61(\mathrm{~s}, 1 \mathrm{H}), 4.41(\mathrm{t}, 2 \mathrm{H}, J=4.8 \mathrm{~Hz}), 3.89-$ $3.98(\mathrm{~m}, 2 \mathrm{H}), 3.74(\mathrm{~s}, 3 \mathrm{H}), 2.07(\mathrm{~s}, 3 \mathrm{H}) .{ }^{13} \mathrm{C} \mathrm{NMR}\left(75 \mathrm{MHz}, \mathrm{CDCl}_{3}, \mathrm{TMS}\right): \delta=166.8,158.5,145.6$, $143.3,138.0,137.0,134.1,130.2,129.5,129.4,128.5,128.0,122.8,120.9,114.7,114.5,110.4,65.5$, 63.0, 55.8, 19.9; HPLC (Daicel CHIRALCEL AD column, hexane $/ \mathrm{PrOH}=65: 35$ at $0.5 \mathrm{~mL} / \mathrm{min}, \lambda=$ $\left.254 \mathrm{~nm}): \mathrm{t}_{\text {major }}=11.75 \mathrm{~min}, \mathrm{t}_{\text {minor }}=20.39 \mathrm{~min}, \mathrm{ee}=90 \% ; \alpha\right]_{\mathrm{D}}{ }^{24.6}($ major $)=+9.1\left(\mathrm{c}=1.01, \mathrm{CHCl}_{3}\right)$.<smiles>COc1ccc(Br)c(-c2c(C)cccc2C(=O)OCCCOc2ccccc2)c1O</smiles>

$3 r$ 
3-(Benzyloxy)propyl $(R)$-6'-bromo-2'-hydroxy-3'-methoxy-6-methyl-[1,1'-biphenyl]-2-carboxylate (3r): White solid; 97\% yield, 96\% ee; ${ }^{1} \mathrm{H}-\mathrm{NMR}\left(300 \mathrm{MHz}, \mathrm{CDCl}_{3}\right): \delta=7.89(\mathrm{~d}, 1 \mathrm{H}, J=7.8 \mathrm{~Hz}), 7.49$ $(\mathrm{d}, 1 \mathrm{H}, J=7.2 \mathrm{~Hz}), 7.38(\mathrm{t}, 1 \mathrm{H}, J=7.5 \mathrm{~Hz}), 7.27-7.34(\mathrm{~m}, 5 \mathrm{H}), 7.15(\mathrm{~d}, 1 \mathrm{H}, J=8.7 \mathrm{~Hz}), 6.75(\mathrm{t}, 1 \mathrm{H}, J$ $=8.7 \mathrm{~Hz}), 5.60(\mathrm{~s}, 1 \mathrm{H}), 4.47(\mathrm{~s}, 3 \mathrm{H}), 4.19(\mathrm{t}, 2 \mathrm{H}, J=6.3 \mathrm{~Hz}), 3.89(\mathrm{~s}, 3 \mathrm{H}), 3.43(\mathrm{t}, 2 \mathrm{H}, J=6.3 \mathrm{~Hz}), 2.07$ $(\mathrm{s}, 3 \mathrm{H}), 3.43(\mathrm{t}, 2 \mathrm{H}, J=6.3 \mathrm{~Hz}), 3.43$ (quint, $2 \mathrm{H}, J=6.3 \mathrm{~Hz}) \cdot{ }^{13} \mathrm{C}$ NMR $\left(75 \mathrm{MHz}, \mathrm{CDCl}_{3}, \mathrm{TMS}\right): \delta=$ $166.9,145.7,143.3,138.4,138.0,136.8,133.8,130.7,128.3,128.2,128.0,127.9,127.6,127.5,122.9$, 114.9, 110.6, 73.0, 66.8, 62.0, 56.0, 28.9, 19.9; HPLC (Daicel CHIRALCEL AD column, hexane/iPrOH $=65: 35$ at $0.5 \mathrm{~mL} / \mathrm{min}, \lambda=254 \mathrm{~nm}$ ): $\mathrm{t}_{\text {major }}=10.70 \mathrm{~min}, \mathrm{t}_{\text {minor }}=18.08 \mathrm{~min}$, ee $=96 \%$; $[\alpha]_{\mathrm{D}}{ }^{25.0}$ (major) $=$ $+8.6\left(\mathrm{c}=1.00, \mathrm{CHCl}_{3}\right)$.

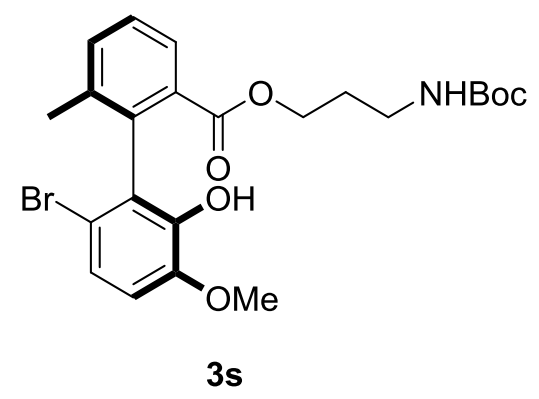

3-((tert-Butoxycarbonyl)amino)propyl

(R)-6'-bromo-2'-hydroxy-3'-methoxy-6-methyl-[1,1'biphenyl]-2-carboxylate (3s): White solid; 95\% yield, 94\% ee; ${ }^{1} \mathrm{H}-\mathrm{NMR}\left(300 \mathrm{MHz}, \mathrm{CDCl}_{3}\right): \delta=7.88$ $\left(\mathrm{dd}, 1 \mathrm{H}, J_{I}=0.6 \mathrm{~Hz}, J_{2}=7.5 \mathrm{~Hz}\right), 7.49\left(\mathrm{dd}, 1 \mathrm{H}, J_{I}=1.2 \mathrm{~Hz}, J_{2}=7.5 \mathrm{~Hz}\right), 7.39(\mathrm{t}, 1 \mathrm{H}, J=7.5 \mathrm{~Hz}), 7.16$ $(\mathrm{d}, 1 \mathrm{H}, J=8.7 \mathrm{~Hz}), 6.78(\mathrm{~d}, 1 \mathrm{H}, J=8.7 \mathrm{~Hz}), 5.91(\mathrm{~s}, 1 \mathrm{H}), 4.65(\mathrm{~s}, 1 \mathrm{H}), 4.10(\mathrm{t}, 2 \mathrm{H}, J=6.0 \mathrm{~Hz}), 3.91(\mathrm{~s}$, $3 \mathrm{H}), 3.04(\mathrm{q}, 2 \mathrm{H}, J=6.3 \mathrm{~Hz}), 2.07(\mathrm{~s}, 3 \mathrm{H}), 1.50-1.68(\mathrm{~m}, 2 \mathrm{H}), 1.43(\mathrm{~s}, 9 \mathrm{H}) .{ }^{13} \mathrm{C} \mathrm{NMR}\left(75 \mathrm{MHz}, \mathrm{CDCl}_{3}\right.$, TMS): $\delta=167.2,155.8,145.9,143.5,138.0,136.8,133.8,130.7,128.0,122.9,114.9,110.7,79.2,62.3$, 56.1, 37.4, 28.9, 18.4, 19.9; HPLC (Daicel CHIRALCEL AD column, hexane/ $i \mathrm{PrOH}=65: 35$ at 0.5 $\mathrm{mL} / \mathrm{min}, \lambda=254 \mathrm{~nm}): \mathrm{t}_{\text {major }}=8.73 \mathrm{~min}, \mathrm{t}_{\text {minor }}=13.76 \mathrm{~min}$, ee $=94 \% ;[\alpha]_{\mathrm{D}}^{25.7}$ (major) $=-7.7(\mathrm{c}=1.00$, $\left.\mathrm{CHCl}_{3}\right)$.<smiles>COc1ccc(Br)c(-c2c(C)cccc2C(=O)OC(COc2ccccc2)COc2ccccc2)c1</smiles>

3t

1,3-Bis(benzyloxy)propan-2-yl $\quad(R)$-6' -bromo-2'-hydroxy-3'-methoxy-6-methyl-[1,1'-biphenyl]-2carboxylate (3t): colorless oil; 80\% yield, 99\% ee; ${ }^{1} \mathrm{H}-\mathrm{NMR}\left(300 \mathrm{MHz}, \mathrm{CDCl}_{3}\right): \delta=7.94\left(\mathrm{dd}, 1 \mathrm{H}, J_{1}=\right.$ $\left.0.9 \mathrm{~Hz}, J_{2}=7.8 \mathrm{~Hz}\right), 7.50\left(\mathrm{dd}, 1 \mathrm{H}, J_{1}=0.6 \mathrm{~Hz}, J_{2}=7.5 \mathrm{~Hz}\right), 7.40(\mathrm{t}, 1 \mathrm{H}, J=7.5 \mathrm{~Hz}), 7.26-7.35(\mathrm{~m}, 10 \mathrm{H})$, $7.10(\mathrm{~d}, 1 \mathrm{H}, J=8.7 \mathrm{~Hz}), 6.69(\mathrm{~d}, 1 \mathrm{H}, J=8.7 \mathrm{~Hz}), 5.56(\mathrm{~s}, 1 \mathrm{H}), 5.25$ (quint, $1 \mathrm{H}, J=5.1 \mathrm{~Hz}$ ), 4.47-4.52 (m, 4H), $3.83(\mathrm{~s}, 3 \mathrm{H}), 3.43-3.58(\mathrm{~m}, 4 \mathrm{H}), 2.07(\mathrm{~s}, 3 \mathrm{H}) .{ }^{13} \mathrm{C} \mathrm{NMR}\left(75 \mathrm{MHz}, \mathrm{CDCl}_{3}, \mathrm{TMS}\right): \delta=166.2$, $145.8,143.4,138.2$, 138.0, 136.9, 133.9, 130.7, 128.3, 128.2, 128.0, 127.9, 127.6, 127.5, 122.9, 114.9, 110.5, 68.5, 68.4, 56.0, 19.9; HPLC (Daicel CHIRALCEL AD column, hexane/ $i \mathrm{PrOH}=75: 25$ at 0.5 $\mathrm{mL} / \mathrm{min}, \lambda=254 \mathrm{~nm}): \mathrm{t}_{\text {major }}=14.43 \mathrm{~min}, \mathrm{t}_{\text {minor }}=25.05 \mathrm{~min}, \mathrm{ee}=99 \% ;[\alpha]_{\mathrm{D}}^{25.6}$ (major) $=+6.5(\mathrm{c}=1.00$, $\left.\mathrm{CHCl}_{3}\right)$. 


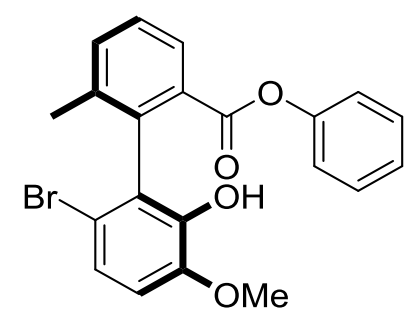

$3 \mathbf{u}$

Phenyl $(R)-6$ '-bromo-2'-hydroxy-3'-methoxy-6-methyl-[1,1'-biphenyl]-2-carboxylate (3u): White solid; $75 \%$ yield, $96 \%$ ee; ${ }^{1} \mathrm{H}-\mathrm{NMR}\left(300 \mathrm{MHz}, \mathrm{CDCl}_{3}\right): \delta=8.10(\mathrm{~d}, 1 \mathrm{H}, J=7.5 \mathrm{~Hz}), 7.57(\mathrm{~d}, 1 \mathrm{H}, J=$ $7.5 \mathrm{~Hz}), 7.47(\mathrm{t}, 1 \mathrm{H}, J=7.8 \mathrm{~Hz}), 7.31(\mathrm{t}, 2 \mathrm{H}, J=7.2 \mathrm{~Hz}), 7.15-7.18(\mathrm{~m}, 2 \mathrm{H}), 6.97(\mathrm{~d}, 2 \mathrm{H}, J=7.8 \mathrm{~Hz})$, $6.74(\mathrm{~d}, 2 \mathrm{H}, J=8.7 \mathrm{~Hz}), 5.67(\mathrm{~s}, 1 \mathrm{H}), 3.88(\mathrm{~s}, 3 \mathrm{H}), 2.13(\mathrm{~s}, 3 \mathrm{H}) .{ }^{13} \mathrm{C} \mathrm{NMR}\left(75 \mathrm{MHz}, \mathrm{CDCl}_{3}, \mathrm{TMS}\right): \delta=$ 165.3, 150.9, 145.8, 143.4, 138.3, 137.5, 134.4, 130.0, 129.2, 128.5, 128.1, 127.4, 125.5, 123.1, 121.6, 114.7, 110.8, 56.0, 19.9; HPLC (Daicel CHIRALCEL AD column, hexane/ $i \operatorname{PrOH}=75: 25$ at 0.5 $\mathrm{mL} / \mathrm{min}, \lambda=210 \mathrm{~nm}): \mathrm{t}_{\text {major }}=15.93 \mathrm{~min}, \mathrm{t}_{\mathrm{minor}}=26.88 \mathrm{~min}$, ee $=96 \% ;[\alpha]_{\mathrm{D}}^{25.0}$ (major) $=+7.9(\mathrm{c}=1.00$, $\left.\mathrm{CHCl}_{3}\right)$.<smiles>COc1ccc(Br)c(-c2c(C)cccc2C(=O)Oc2ccc(S(C)(=O)=O)cc2)c1O</smiles>

3v

4-(Methylthio)phenyl (R)-6'-bromo-2'-hydroxy-3'-methoxy-6-methyl-[1,1'-biphenyl]-2-carboxylate (3v): White solid; $60 \%$ yield, 93\% ee; ${ }^{1} \mathrm{H}-\mathrm{NMR}\left(300 \mathrm{MHz}, \mathrm{CDCl}_{3}\right): \delta=8.07\left(\mathrm{dd}, 1 \mathrm{H}, J_{1}=0.9 \mathrm{~Hz}, J_{2}=\right.$ $7.5 \mathrm{~Hz}), 7.56(\mathrm{~d}, 1 \mathrm{H}, J=7.5 \mathrm{~Hz}), 7.46(\mathrm{t}, 1 \mathrm{H}, J=7.8 \mathrm{~Hz}), 7.21(\mathrm{~d}, 2 \mathrm{H}, J=8.7 \mathrm{~Hz}), 7.16(\mathrm{~d}, 1 \mathrm{H}, J=8.7$ $\mathrm{Hz}), 6.90(\mathrm{~d}, 2 \mathrm{H}, J=8.7 \mathrm{~Hz}), 6.74(\mathrm{~d}, 1 \mathrm{H}, J=8.7 \mathrm{~Hz}), 5.67(\mathrm{~s}, 1 \mathrm{H}), 3.88(\mathrm{~s}, 3 \mathrm{H}), 2.44(\mathrm{~s}, 3 \mathrm{H}), 2.12(\mathrm{~s}$, $3 \mathrm{H}) .{ }^{13} \mathrm{C} \mathrm{NMR}\left(75 \mathrm{MHz}, \mathrm{CDCl}_{3}\right.$, TMS): $\delta=165.3,148.7,145.8,143.4,138.4,137.5,135.3,134.5$, 129.9, 128.5, 128.2, 127.4, 123.1, 122.0, 114.7, 110.8, 56.1, 19.9, 16.6; HPLC (Daicel CHIRALCEL $\mathrm{AD}$ column, hexane $/ \mathrm{PrOH}=75: 25$ at $0.5 \mathrm{~mL} / \mathrm{min}, \lambda=210 \mathrm{~nm}): \mathrm{t}_{\text {major }}=19.87 \mathrm{~min}, \mathrm{t}_{\text {minor }}=37.23 \mathrm{~min}$, ee $=93 \% ;[\alpha]_{\mathrm{D}}^{25.0}$ (major) $=+15.4\left(\mathrm{c}=1.00, \mathrm{CHCl}_{3}\right)$.<smiles>COc1ccc(Br)c(-c2c(C)cccc2C(=O)Oc2ccc(Br)cc2)c1O</smiles>

$3 w$

4-Bromophenyl $(R)-6$ '-bromo-2'-hydroxy-3'-methoxy-6-methyl-[1,1'-biphenyl]-2-carboxylate (3w): White solid; $65 \%$ yield, $90 \%$ ee; ${ }^{1} \mathrm{H}-\mathrm{NMR}\left(300 \mathrm{MHz}, \mathrm{CDCl}_{3}\right): \delta=8.07\left(\mathrm{dd}, 1 \mathrm{H}, J_{1}=0.9 \mathrm{~Hz}, J_{1}=7.8\right.$ $\mathrm{Hz}), 7.57\left(\mathrm{dd}, 1 \mathrm{H}, J_{1}=0.6 \mathrm{~Hz}, J_{1}=7.5 \mathrm{~Hz}\right), 7.48(\mathrm{~d}, 1 \mathrm{H}, J=7.5 \mathrm{~Hz}), 7.40-7.44(\mathrm{~m}, 2 \mathrm{H}), 7.16(\mathrm{~d}, 1 \mathrm{H}, J$ $=8.7 \mathrm{~Hz}), 6.84-6.87(\mathrm{~m}, 2 \mathrm{H}), 6.75(\mathrm{~d}, 1 \mathrm{H}, J=8.7 \mathrm{~Hz}), 5.67(\mathrm{~s}, 1 \mathrm{H}), 3.89(\mathrm{~s}, 3 \mathrm{H}), 2.12(\mathrm{~s}, 3 \mathrm{H}) .{ }^{13} \mathrm{C}$ NMR 
(75 MHz, $\left.\mathrm{CDCl}_{3}, \mathrm{TMS}\right): \delta=165.0,150.0,145.8,143.4,138.5,137.6,134.6,132.3,129.5,128.5,128.2$ 127.3, 123.4, 123.0, 118.6, 114.6, 110.8, 56.1, 19.9; HPLC (Daicel CHIRALCEL AD column, hexane $/ \mathrm{PrOH}=75: 25$ at $0.5 \mathrm{~mL} / \mathrm{min}, \lambda=210 \mathrm{~nm}): \mathrm{t}_{\text {major }}=17.31 \mathrm{~min}, \mathrm{t}_{\text {minor }}=35.81 \mathrm{~min}$, ee $=90 \%$; $[\alpha]_{\mathrm{D}}^{25.1}($ major $)=13.8\left(\mathrm{c}=1.00, \mathrm{CHCl}_{3}\right)$.<smiles>COc1ccc(Br)c(-c2c(C)cccc2C(=O)Oc2cc(C)cc(C)c2)c1O</smiles>

$3 x$

3,5-Dimethylphenyl $(R)-6$ '-bromo-2'-hydroxy-3'-methoxy-6-methyl-[1,1'-biphenyl]-2-carboxylate (3x): White solid; $76 \%$ yield, $98 \%$ ee; ${ }^{1} \mathrm{H}-\mathrm{NMR}\left(300 \mathrm{MHz}, \mathrm{CDCl}_{3}\right): \delta=8.07\left(\mathrm{dd}, 1 \mathrm{H}, J_{1}=0.6 \mathrm{~Hz}, J_{2}=\right.$ $7.5 \mathrm{~Hz}), 7.56\left(\mathrm{dd}, 1 \mathrm{H}, J_{l}=0.6 \mathrm{~Hz}, J_{l}=7.5 \mathrm{~Hz}\right), 7.46(\mathrm{~d}, 1 \mathrm{H}, J=7.5 \mathrm{~Hz}), 7.16(\mathrm{t}, 1 \mathrm{H}, J=8.7 \mathrm{~Hz}), 6.80$ $(\mathrm{d}, 1 \mathrm{H}, J=0.6 \mathrm{~Hz}), 6.74(\mathrm{~d}, 2 \mathrm{H}, J=8.7 \mathrm{~Hz}), 6.58(\mathrm{~s}, 2 \mathrm{H}), 5.66(\mathrm{~s}, 1 \mathrm{H}), 3.88(\mathrm{~s}, 3 \mathrm{H}), 2.26(\mathrm{~s}, 6 \mathrm{H}), 2.12(\mathrm{~s}$, $3 \mathrm{H}) .{ }^{13} \mathrm{C}$ NMR $\left(75 \mathrm{MHz}, \mathrm{CDCl}_{3}, \mathrm{TMS}\right): \delta=165.4,150.7,145.8,143.4,139.0,138.3,137.4,134.3$, 130.2, 128.4, 128.1, 127.5, 127.3, 123.0, 119.1, 114.8, 110.7, 56.0, 21.2, 19.9; HPLC (Daicel CHIRALCEL AD column, hexane $/ \mathrm{PrOH}=75: 25$ at $0.5 \mathrm{~mL} / \mathrm{min}, \lambda=210 \mathrm{~nm}$ ): $\mathrm{t}_{\text {major }}=11.68 \mathrm{~min}$, $\mathrm{t}_{\text {minor }}$ $=19.69 \min , \mathrm{ee}=98 \% ;[\alpha]_{\mathrm{D}}^{25.0}$ (major) $=+7.0\left(\mathrm{c}=1.00, \mathrm{CHCl}_{3}\right)$.<smiles>COc1ccccc1OC(=O)c1cccc(C)c1-c1c(C)cccc1O</smiles>

3y

o-Tolyl (R)-6'-bromo-2'-hydroxy-3'-methoxy-6-methyl-[1,1'-biphenyl]-2-carboxylate (3y): White solid; 50\% yield, 97\% ee; ${ }^{1} \mathrm{H}-\mathrm{NMR}\left(300 \mathrm{MHz}, \mathrm{CDCl}_{3}\right): \delta=8.14\left(\mathrm{dd}, 1 \mathrm{H}, J_{1}=0.6 \mathrm{~Hz}, J_{2}=7.8 \mathrm{~Hz}\right)$, $7.58\left(\mathrm{dd}, 1 \mathrm{H}, J_{1}=0.6 \mathrm{~Hz}, J_{2}=7.5 \mathrm{~Hz}\right), 7.47(\mathrm{t}, 1 \mathrm{H}, J=7.8 \mathrm{~Hz}), 7.40-7.43(\mathrm{~m}, 1 \mathrm{H}), 7.10-7.16(\mathrm{~m}, 3 \mathrm{H})$, $6.90\left(\mathrm{dd}, 1 \mathrm{H}, J_{1}=1.5 \mathrm{~Hz}, J_{2}=7.5 \mathrm{~Hz}\right), 6.72(\mathrm{t}, 1 \mathrm{H}, J=8.7 \mathrm{~Hz}), 5.65(\mathrm{~s}, 1 \mathrm{H}), 3.87(\mathrm{~s}, 3 \mathrm{H}), 2.12(\mathrm{~s}, 6 \mathrm{H})$. ${ }^{13} \mathrm{C}$ NMR $\left(75 \mathrm{MHz}, \mathrm{CDCl}_{3}\right.$, TMS): $\delta=164.8,149.5,145.8,143.4,138.4,137.8,134.4,130.9,130.5$, 129.6, 128.4, 128.1, 126.8, 125.8, 125.2, 123.1, 121.8, 114.6, 110.8, 56.0, 19.9, 16.1; HPLC (Daicel CHIRALCEL AD column, hexane $/ \mathrm{PrOH}=70: 20$ at $0.5 \mathrm{~mL} / \mathrm{min}, \lambda=210 \mathrm{~nm}): \mathrm{t}_{\text {major }}=16.31 \mathrm{~min}$, $\mathrm{t}_{\text {minor }}$ $=24.16 \mathrm{~min}$, ee $=97 \% ;[\alpha]_{\mathrm{D}}^{25.0}$ (major) $=+4.4\left(\mathrm{c}=1.00, \mathrm{CHCl}_{3}\right)$. 
<smiles>COc1ccc(Br)c(-c2c(C)cc(C)cc2C(=O)OCc2ccc([N+](=O)[O-])cc2)c1O</smiles>

4-Nitrobenzyl $\quad(R)$-6'-bromo-2'-hydroxy-3'-methoxy-4,6-dimethyl-[1,1'-biphenyl]-2-carboxylate (3z): White solid; quantitative yield, $94 \%$ ee; ${ }^{1} \mathrm{H}-\mathrm{NMR}\left(300 \mathrm{MHz}, \mathrm{CDCl}_{3}\right): \delta=8.14(\mathrm{~d}, 2 \mathrm{H}, J=8.7 \mathrm{~Hz})$, $7.77(\mathrm{~s}, 1 \mathrm{H}), 7.31-7.34(\mathrm{~m}, 3 \mathrm{H}), 7.06(\mathrm{~d}, 1 \mathrm{H}, J=8.7 \mathrm{~Hz}), 6.63(\mathrm{~d}, 1 \mathrm{H}, J=8.7 \mathrm{~Hz}), 5.57(\mathrm{~s}, 1 \mathrm{H}), 5.17(\mathrm{~d}$, $2 \mathrm{H}, J=2.7 \mathrm{~Hz}), 3.84(\mathrm{~s}, 3 \mathrm{H}), 2.42(\mathrm{~s}, 3 \mathrm{H}), 2.02(\mathrm{~s}, 3 \mathrm{H}) .{ }^{13} \mathrm{C} \mathrm{NMR}\left(75 \mathrm{MHz}, \mathrm{CDCl}_{3}, \mathrm{TMS}\right): \delta=166.7$, 147.5, 145.6, 143.3, 143.1, 138.0, 135.2, 134.0, 129.8, 128.9, 128.5, 127.6, 123.5, 122.9, 115.0, 110.3, 65.1, 55.9, 21.1, 19.8; HPLC (Daicel CHIRALCEL AS-H column, hexane $/ \mathrm{PrOH}=75: 25$ at $0.5 \mathrm{~mL} / \mathrm{min}$, $\lambda=254 \mathrm{~nm}): \mathrm{t}_{\text {major }}=22.98 \mathrm{~min}, \mathrm{t}_{\text {minor }}=36.17 \mathrm{~min}, \mathrm{ee}=94 \% ;[\alpha]_{\mathrm{D}}^{25.4}($ major $)=+12.6\left(\mathrm{c}=1.00, \mathrm{CHCl}_{3}\right)$.<smiles>CCc1cccc(C(=O)OCc2ccc([N+](=O)[O-])cc2)c1-c1c(Br)ccc(OC)c1O</smiles>

4-Nitrobenzyl $(R)$-6'-bromo-6-ethyl-2'-hydroxy-3'-methoxy-[1,1'-biphenyl]-2-carboxylate (3aa): White solid; 97\% yield, 97\% ee; ${ }^{1} \mathrm{H}-\mathrm{NMR}\left(300 \mathrm{MHz}, \mathrm{CDCl}_{3}\right): \delta=8.15(\mathrm{~d}, 2 \mathrm{H}, J=8.7 \mathrm{~Hz}), 7.95(\mathrm{~d}, 1 \mathrm{H}$, $J=7.5 \mathrm{~Hz}), 7.57(\mathrm{~d}, 1 \mathrm{H}, J=6.9 \mathrm{~Hz}), 7.47(\mathrm{t}, 1 \mathrm{H}, J=7.8 \mathrm{~Hz}), 7.35(\mathrm{~d}, 2 \mathrm{H}, J=8.4 \mathrm{~Hz}), 7.06(\mathrm{~d}, 1 \mathrm{H}, J=$ $8.7 \mathrm{~Hz}), 6.64(\mathrm{~d}, 1 \mathrm{H}, J=8.7 \mathrm{~Hz}), 5.59(\mathrm{~s}, 2 \mathrm{H}), 5.13-5.23(\mathrm{~m}, 2 \mathrm{H}), 3.85(\mathrm{~s}, 3 \mathrm{H}), 2.37(\mathrm{q}, 2 \mathrm{H}, J=7.5 \mathrm{~Hz})$, $1.08(\mathrm{t}, 3 \mathrm{H}, J=7.8 \mathrm{~Hz}) .{ }^{13} \mathrm{C}$ NMR $\left(75 \mathrm{MHz}, \mathrm{CDCl}_{3}, \mathrm{TMS}\right): \delta=166.6,147.5,145.5,143.9,143.5,143.1$, 136.3, 132.6, 130.0, 128.4, 128.2, 127.2, 123.5, 122.9, 115.2, 110.4, 65.1, 55.9, 26.1, 14.4; HPLC (Daicel CHIRALCEL AS-H column, hexane $/ \mathrm{PrOH}=85: 15$ at $0.5 \mathrm{~mL} / \mathrm{min}, \lambda=254 \mathrm{~nm}$ ): $\mathrm{t}_{\text {major }}=29.96$ min, $\mathrm{t}_{\text {minor }}=55.29$ min, ee $=97 \% ;[\alpha]_{\mathrm{D}}^{25.2}($ major $)=+5.6\left(\mathrm{c}=1.00, \mathrm{CHCl}_{3}\right)$.<smiles>COc1ccc(Br)c(-c2c(Br)cccc2C(=O)OCc2ccc([N+](=O)[O-])cc2)c1O</smiles>

$3 a b$

4-Nitrobenzyl $(R)$-6-benzyl-6'-bromo-2'-hydroxy-3'-methoxy-[1,1'-biphenyl]-2-carboxylate (3ab): White solid; 94\% yield, 96\% ee; ${ }^{1} \mathrm{H}-\mathrm{NMR}\left(300 \mathrm{MHz}, \mathrm{CDCl}_{3}\right): \delta=8.14(\mathrm{~d}, 2 \mathrm{H}, J=8.4 \mathrm{~Hz}), 7.96(\mathrm{~d}, 1 \mathrm{H}$, $J=7.2 \mathrm{~Hz}), 7.33-7.45(\mathrm{~m}, 4 \mathrm{H}), 7.14-7.21(\mathrm{~m}, 3 \mathrm{H}), 7.06(\mathrm{~d}, 1 \mathrm{H}, J=8.7 \mathrm{~Hz}), 6.98(\mathrm{~d}, 1 \mathrm{H}, J=6.6 \mathrm{~Hz})$, $6.63(\mathrm{~d}, 1 \mathrm{H}, J=8.7 \mathrm{~Hz}), 5.13-5.24(\mathrm{~m}, 2 \mathrm{H}), 3.83(\mathrm{~s}, 3 \mathrm{H}), 3.71(\mathrm{~d}, 2 \mathrm{H}, J=2.1 \mathrm{~Hz}) .{ }^{13} \mathrm{C}$ NMR $(75 \mathrm{MHz}$, 
$\left.\mathrm{CDCl}_{3}, \mathrm{TMS}\right): \delta=166.7,147.5,145.6,143.3,143.1,138.0,135.2,134.0,129.8,128.9,128.5,127.6$, 123.5, 122.9, 115.0, 110.3, 65.1, 55.9, 21.1, 19.8; HPLC (Daicel CHIRALCEL AD column, hexane $/ \mathrm{PrOH}=75: 25$ at $0.5 \mathrm{~mL} / \mathrm{min}, \lambda=254 \mathrm{~nm}): \mathrm{t}_{\text {major }}=27.63 \mathrm{~min}, \mathrm{t}_{\text {minor }}=57.39 \mathrm{~min}$, ee $=95 \%$; $[\alpha]_{\mathrm{D}}^{25.5}$ (major) $=+19.0\left(\mathrm{c}=1.00, \mathrm{CHCl}_{3}\right)$.<smiles>COc1ccc(Br)c(-c2c(C(=O)OCc3ccc([N+](=O)[O-])cc3)cccc2C(C)C)c1O</smiles>

4-Nitrobenzyl $\quad(R)-6$ '-bromo-2'-hydroxy-6-isopropyl-3'-methoxy-[1,1'-biphenyl]-2-carboxylate (3ac): White solid; 92\% yield, 94\% ee; ${ }^{1} \mathrm{H}-\mathrm{NMR}\left(300 \mathrm{MHz}, \mathrm{CDCl}_{3}\right): \delta=8.15(\mathrm{~d}, 2 \mathrm{H}, J=8.7 \mathrm{~Hz}), 7.92$ $\left(\mathrm{dd}, 1 \mathrm{H}, J_{l}=1.2 \mathrm{~Hz}, J_{2}=7.8 \mathrm{~Hz}\right), 7.64\left(\mathrm{dd}, 1 \mathrm{H}, J_{1}=1.2 \mathrm{~Hz}, J_{2}=7.8 \mathrm{~Hz}\right), 7.51(\mathrm{t}, 1 \mathrm{H}, J=7.8 \mathrm{~Hz}), 7.36$ $(\mathrm{d}, 2 \mathrm{H}, J=8.7 \mathrm{~Hz}), 7.06(\mathrm{~d}, 1 \mathrm{H}, J=8.7 \mathrm{~Hz}), 6.65(\mathrm{~d}, 1 \mathrm{H}, J=8.7 \mathrm{~Hz}), 5.59(\mathrm{~s}, 1 \mathrm{H}), 5.12-5.23(\mathrm{~m}, 2 \mathrm{H})$, $3.85(\mathrm{~s}, 3 \mathrm{H}), 2.58-2.67(\mathrm{~m}, 1 \mathrm{H}), 1.17(\mathrm{~d}, 3 \mathrm{H}, J=6.9 \mathrm{~Hz}), 1.11(\mathrm{~d}, 3 \mathrm{H}, J=6.9 \mathrm{~Hz}) .{ }^{13} \mathrm{C} \mathrm{NMR}(75 \mathrm{MHz}$, $\left.\mathrm{CDCl}_{3}, \mathrm{TMS}\right): \delta=166.7,148.8,147.5,145.5,143.8,143.2,135.3,130.1,130.0,128.6,128.4,128.1$, 127.1, 123.5, 122.8, 115.5, 110.4, 65.1, 55.9, 30.3, 24.1, 23.6; HPLC (Daicel CHIRALCEL AD column, hexane $/ \mathrm{PrOH}=65: 35$ at $0.5 \mathrm{~mL} / \mathrm{min}, \lambda=254 \mathrm{~nm}): \mathrm{t}_{\text {major }}=13.33 \mathrm{~min}, \mathrm{t}_{\text {minor }}=31.76 \mathrm{~min}$, ee $=94 \%$; $[\alpha]_{\mathrm{D}}^{25.1}$ (major) $=+4.1\left(\mathrm{c}=1.00, \mathrm{CHCl}_{3}\right)$.<smiles>COc1ccc(Br)c(-c2c(C(=O)OCc3ccc([N+](=O)[O-])cc3)ccc3c2CCCC3)c1O</smiles>

\section{3ad}

4-Nitrobenzyl

(R)-1-(6-bromo-2-hydroxy-3-methoxyphenyl)-5,6,7,8-tetrahydronaphthalene-2carboxylate (3ad): White solid; quantitative yield, $92 \%$ ee; ${ }^{1} \mathrm{H}-\mathrm{NMR}\left(300 \mathrm{MHz}, \mathrm{CDCl}_{3}\right): \delta=8.14(\mathrm{~d}$, $2 \mathrm{H}, J=8.7 \mathrm{~Hz}), 7.88(\mathrm{~d}, 1 \mathrm{H}, J=8.1 \mathrm{~Hz}), 7.38(\mathrm{~d}, 2 \mathrm{H}, J=8.7 \mathrm{~Hz}), 7.24(\mathrm{~d}, 1 \mathrm{H}, J=8.1 \mathrm{~Hz}), 7.06(\mathrm{~d}, 1 \mathrm{H}$, $J=8.7 \mathrm{~Hz}), 6.63(\mathrm{~d}, 1 \mathrm{H}, J=8.7 \mathrm{~Hz}), 5.12-5.23(\mathrm{~m}, 2 \mathrm{H}), 3.85(\mathrm{~s}, 3 \mathrm{H}), 2.89(\mathrm{t}, 2 \mathrm{H}, J=5.7 \mathrm{~Hz}), 2.29(\mathrm{t}$, $2 \mathrm{H}, J=6.0 \mathrm{~Hz}), 1.71-1.77(\mathrm{~m}, 4 \mathrm{H}) .{ }^{13} \mathrm{C} \mathrm{NMR}\left(75 \mathrm{MHz}, \mathrm{CDCl}_{3}, \mathrm{TMS}\right): \delta=166.4,147.5,145.6,143.3$, 143.1, 143.0, 137.3, 137.0, 129.4, 128.3, 127.6, 126.9, 123.4, 122.9, 114.7, 110.3, 64.9, 55.9, 30.4, 26.8, 22.9, 22.3; HPLC (Daicel CHIRALCEL AS-H column, hexane $/ \mathrm{PrOH}=75: 25$ at $0.5 \mathrm{~mL} / \mathrm{min}, \lambda=254$ $\mathrm{nm}): \mathrm{t}_{\text {major }}=27.18 \mathrm{~min}, \mathrm{t}_{\text {minor }}=52.71 \mathrm{~min}, \mathrm{ee}=92 \% ;[\alpha]_{\mathrm{D}}^{25.6}($ major $)=+19.4\left(\mathrm{c}=1.00, \mathrm{CHCl}_{3}\right)$. 


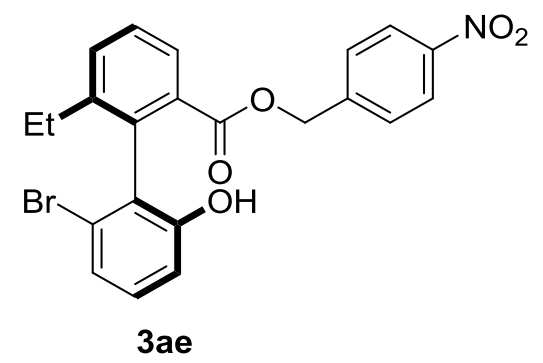

4-Nitrobenzyl $(\boldsymbol{R})$-2' -bromo-6-ethyl-6'-hydroxy-[1,1'-biphenyl]-2-carboxylate (3ae): White solid; 97\% yield, 97\% ee; ${ }^{1} \mathrm{H}-\mathrm{NMR}\left(300 \mathrm{MHz}, \mathrm{CDCl}_{3}\right): \delta=8.16(\mathrm{~d}, 2 \mathrm{H}, J=8.7 \mathrm{~Hz}), 7.94(\mathrm{~d}, 1 \mathrm{H}, J=7.5 \mathrm{~Hz}), 7.61$ $(\mathrm{d}, 1 \mathrm{H}, J=7.2 \mathrm{~Hz}), 7.53(\mathrm{t}, 1 \mathrm{H}, J=7.8 \mathrm{~Hz}), 7.34(\mathrm{~d}, 2 \mathrm{H}, J=8.4 \mathrm{~Hz}), 7.14(\mathrm{~d}, 1 \mathrm{H}, J=7.8 \mathrm{~Hz}), 7.07(\mathrm{~d}$, $1 \mathrm{H}, J=8.1 \mathrm{~Hz}), 6.86(\mathrm{~d}, 1 \mathrm{H}, J=8.1 \mathrm{~Hz}), 5.18(\mathrm{~s}, 2 \mathrm{H}), 4.61(\mathrm{~s}, 1 \mathrm{H}), 2.33-2.41(\mathrm{~m}, 2 \mathrm{H}), 1.10(\mathrm{t}, 3 \mathrm{H}, J=$ $7.8 \mathrm{~Hz}) .{ }^{13} \mathrm{C}$ NMR $\left(75 \mathrm{MHz}, \mathrm{CDCl}_{3}\right.$, TMS): $\delta=166.5,153.7,145.1,142.7,134.2,133.1,131.4,129.9$, 129.4, 128.6, 128.5, 124.6, 123.8, 123.6, 114.4, 65.5, 26.1, 14.5; HPLC (Daicel CHIRALCEL AS-H column, hexane $/ \mathrm{iPrOH}=80: 20$ at $0.5 \mathrm{~mL} / \mathrm{min}, \lambda=254 \mathrm{~nm}): \mathrm{t}_{\text {major }}=17.47 \mathrm{~min}, \mathrm{t}_{\text {minor }}=22.42 \mathrm{~min}$, ee $=$ $62 \% ;[\alpha]_{\mathrm{D}}^{25.1}$ (major) $=+4.1\left(\mathrm{c}=1.00, \mathrm{CHCl}_{3}\right)$.<smiles>CCc1cccc(C(=O)OC(COc2ccccc2)COc2ccccc2)c1-c1c(O)cccc1Br</smiles>

\section{3ag}

1,3-Bis(benzyloxy)propan-2-yl $\quad(R)$-2'-bromo-6-ethyl-6'-hydroxy-[1,1'-biphenyl]-2-carboxylate (3ag): colorless oil; $84 \%$ yield, 96\% ee; ${ }^{1} \mathrm{H}-\mathrm{NMR}\left(300 \mathrm{MHz}, \mathrm{CDCl}_{3}\right): \delta=7.91\left(\mathrm{dd}, 1 \mathrm{H}, J_{1}=1.2 \mathrm{~Hz}, J_{2}\right.$ $=7.5 \mathrm{~Hz}), 7.57(\mathrm{~d}, 1 \mathrm{H}, J=7.8 \mathrm{~Hz}), 7.50(\mathrm{~d}, 1 \mathrm{H}, J=7.8 \mathrm{~Hz}), 7.26-7.32(\mathrm{~m}, 10 \mathrm{H}), 7.14\left(\mathrm{dd}, 1 \mathrm{H}, J_{l}=1.2\right.$ $\left.\mathrm{Hz}, J_{2}=8.1 \mathrm{~Hz}\right), 7.06(\mathrm{t}, 1 \mathrm{H}, J=8.1 \mathrm{~Hz}), 6.81\left(\mathrm{dd}, 1 \mathrm{H}, J_{l}=0.9 \mathrm{~Hz}, J_{2}=7.8 \mathrm{~Hz}\right), 5.20-5.27(\mathrm{~m}, 1 \mathrm{H})$, $4.46(\mathrm{~s}, 4 \mathrm{H}), 3.39-3.56(\mathrm{~m}, 4 \mathrm{H}), 2.31-2.40(\mathrm{~m}, 2 \mathrm{H}), 1.09(\mathrm{t}, 3 \mathrm{H}, J=7.8 \mathrm{~Hz}) .{ }^{13} \mathrm{C}$ NMR $\left(75 \mathrm{MHz}, \mathrm{CDCl}_{3}\right.$, TMS): $\delta=166.5,154.0,144.7,138.0,134.1,132.6,132.1,129.7,129.2,128.6,128.3,128.2,127.6$, 124.6, 123.9, 114.7, 73.2, 72.0, 68.6, 68.3, 26.1, 14.5; HPLC (Daicel CHIRALCEL AS-H column, hexane $/ \mathrm{iPrOH}=85: 15$ at $0.5 \mathrm{~mL} / \mathrm{min}, \lambda=254 \mathrm{~nm}): \mathrm{t}_{\text {major }}=10.88 \mathrm{~min}, \mathrm{t}_{\mathrm{minor}}=15.38 \mathrm{~min}$, ee $=96 \%$; $[\alpha]_{\mathrm{D}}^{25.3}$ (major) $=-4.5\left(\mathrm{c}=1.00, \mathrm{CHCl}_{3}\right)$.<smiles>Cc1cc(C)c(-c2c(C(=O)OC(COCc3ccccc3)COc3ccccc3)ccc3ccccc23)c(O)c1</smiles>

3ah

1,3-Bis(benzyloxy)propan-2-yl ( $S$ )-1-(2-hydroxy-4,6-dimethylphenyl)-2-naphthoate (3ah): colorless oil; 56\% yield, 90\% ee; ${ }^{1} \mathrm{H}-\mathrm{NMR}\left(300 \mathrm{MHz}, \mathrm{CDCl}_{3}\right): \delta=7.92-8.04(\mathrm{~m}, 4 \mathrm{H}), 7.58\left(\mathrm{dd}, 1 \mathrm{H}, J_{1}=1.2 \mathrm{~Hz}\right.$, 
$\left.J_{2}=8.1 \mathrm{~Hz}\right), 7.43-7.50(\mathrm{~m}, 2 \mathrm{H}), 7.29-7.36(\mathrm{~m}, 10 \mathrm{H}), 7.50(\mathrm{~d}, 1 \mathrm{H}, J=7.8 \mathrm{~Hz}), 7.26-7.32(\mathrm{~m}, 10 \mathrm{H}), 6.68$ (s, 1H), $6.62(\mathrm{~s}, 1 \mathrm{H}), 5.29-5.34(\mathrm{~m}, 1 \mathrm{H}), 4.43-4.48(\mathrm{~m}, 5 \mathrm{H}), 3.39-3.56(\mathrm{~m}, 4 \mathrm{H}), 2.35(\mathrm{~s}, 3 \mathrm{H}), 1.75(\mathrm{~s}, 3 \mathrm{H})$. ${ }^{13} \mathrm{C}$ NMR $\left(75 \mathrm{MHz}, \mathrm{CDCl}_{3}\right.$, TMS): $\delta=167.3,153.0,138.6,138.0,137.7,135.1,134.9,132.4,130.0$, 128.6, 128.3, 128.1, 128.0, 127.6, 127.3, 126.6, 125.9, 123.0, 122.4, 113.8, 73.1, 72.0, 68.5, 68.4, 21.3, 19.7; HPLC (Daicel CHIRALCEL AS-H column, hexane $/ \mathrm{iPrOH}=80: 20$ at $0.5 \mathrm{~mL} / \mathrm{min}, \lambda=254 \mathrm{~nm}$ ): $\mathrm{t}_{\text {major }}=10.11 \mathrm{~min}, \mathrm{t}_{\text {minor }}=13.91 \mathrm{~min}, \mathrm{ee}=90 \% ;[\alpha]_{\mathrm{D}}^{25.2}($ major $)=+14.6\left(\mathrm{c}=1.00, \mathrm{CHCl}_{3}\right)$.<smiles>Cc1cc(C)c(-c2c(O)c(Br)cc3ccccc23)c(C(=O)OC(COCc2ccccc2)COc2ccccc2)c1</smiles>

1,3-Bis(benzyloxy)propan-2-yl (S)-2-(3-bromo-2-hydroxynaphthalen-1-yl)-3,5-dimethylbenzoate (3ai): colorless oil; $71 \%$ yield, $96 \%$ ee; ${ }^{1} \mathrm{H}-\mathrm{NMR}\left(300 \mathrm{MHz}, \mathrm{CDCl}_{3}\right): \delta=7.98(\mathrm{~s}, 1 \mathrm{H}), 7.77(\mathrm{~s}, 1 \mathrm{H})$, 7.62-7.65 (m, 1H), 7.38-7.39 (m, 1H), 7.20-7.34 (m, 11H), 7.13-7.16 (m, 2H), 7.03-7.06 (m, 1H), $5.42(\mathrm{~s}$, $1 \mathrm{H}), 5.02$ (quint, $1 \mathrm{H}, 5.4 \mathrm{~Hz}), 4.37(\mathrm{~s}, 2 \mathrm{H}), 4.18(\mathrm{~s}, 2 \mathrm{H}), 3.23\left(\mathrm{dd}, 1 \mathrm{H}, J_{1}=4.5 \mathrm{~Hz}, J_{2}=10.5 \mathrm{~Hz}\right), 3.14$ $\left(\mathrm{dd}, 1 \mathrm{H}, J_{l}=5.7 \mathrm{~Hz}, J_{2}=10.5 \mathrm{~Hz}\right), 3.03\left(\mathrm{dd}, 1 \mathrm{H}, J_{l}=5.4 \mathrm{~Hz}, J_{2}=10.2 \mathrm{~Hz}\right), 2.81\left(\mathrm{dd}, 1 \mathrm{H}, J_{l}=5.7 \mathrm{~Hz}\right.$, $\left.J_{2}=10.2 \mathrm{~Hz}\right), 2.47(\mathrm{~s}, 3 \mathrm{H}), 1.92(\mathrm{~s}, 3 \mathrm{H}) .{ }^{13} \mathrm{C}$ NMR $\left(75 \mathrm{MHz}, \mathrm{CDCl}_{3}, \mathrm{TMS}\right): \delta=166.9,146.2,139.0$, 138.4, 138.0, 135.0, 132.6, 132.5, 131.0, 130.8, 129.4, 129.2, 128.3, 128.2, 127.6, 127.4, 127.0, 126.8, 124.4, 124.2, 121.8, 112.2, 73.1, 72.9, 71.6, 68.3, 67.9, 21.1, 19.8; HPLC (Daicel CHIRALCEL AS-H column, hexane $/ \mathrm{iPrOH}=97: 3$ at $0.4 \mathrm{~mL} / \mathrm{min}, \lambda=254 \mathrm{~nm}): \mathrm{t}_{\text {major }}=55.96 \mathrm{~min}, \mathrm{t}_{\text {minor }}=42.98 \mathrm{~min}$, ee $=$ $96 \% ;[\alpha]_{\mathrm{D}}^{25.5}($ major $)=+3.3\left(\mathrm{c}=1.00, \mathrm{CHCl}_{3}\right)$.<smiles>Cc1cc(C)c(-c2c(O)ccc3ccccc23)c(C(=O)OC(COCc2ccccc2)COc2ccccc2)c1</smiles>

3aj

1,3-Bis(benzyloxy)propan-2-yl (S)-2-(2-hydroxynaphthalen-1-yl)-3,5-dimethylbenzoate (3aj): colorless oil; $57 \%$ yield, 93\% ee; ${ }^{1} \mathrm{H}-\mathrm{NMR}\left(300 \mathrm{MHz}, \mathrm{CDCl}_{3}\right): \delta=7.68-7.74(\mathrm{~m}, 3 \mathrm{H}), 7.38(\mathrm{~s}, 1 \mathrm{H})$, 7.11-7.32 (m, 13H), 7.00-7.03 (m, 1H), 5.00 (quint, 1H, 5.4 Hz), 4.26-4.35 (m, 2H), $4.17(\mathrm{~s}, 2 \mathrm{H}), 3.07-$ $3.19(\mathrm{~m}, 2 \mathrm{H}), 2.95-3.00(\mathrm{~m}, 1 \mathrm{H}), 2.77-2.82(\mathrm{~m}, 1 \mathrm{H}), 2.45(\mathrm{~s}, 3 \mathrm{H}), 1.88(\mathrm{~s}, 3 \mathrm{H}) .{ }^{13} \mathrm{C}$ NMR $(75 \mathrm{MHz}$, $\left.\mathrm{CDCl}_{3}, \mathrm{TMS}\right): \delta=167.3,150.1,139.8,138.6,134.9,133.6,133.2,130.0,129.1,129.0,128.9,128.2$, 127.9, 127.5, 127.4, 126.5, 124.0, 123.2, 119.6, 117.6, 73.0, 72.9, 71.7, 68.3, 67.9, 21.1, 19.7; HPLC (Daicel CHIRALCEL AS-H column, hexane $/ \mathrm{PrOH}=85: 15$ at $0.5 \mathrm{~mL} / \mathrm{min}, \lambda=254 \mathrm{~nm}$ ): $\mathrm{t}_{\text {major }}=12.13$ min, $\mathrm{t}_{\text {minor }}=17.30 \mathrm{~min}$, ee $=93 \% ;[\alpha]_{\mathrm{D}}^{25.5}$ (major) $=-28.9\left(\mathrm{c}=1.00, \mathrm{CHCl}_{3}\right)$. 


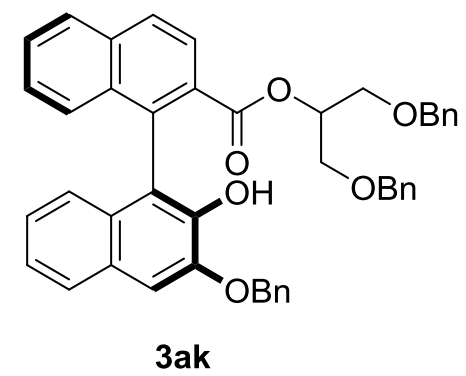

1,3-Bis(benzyloxy)propan-2-yl $\quad(S)$-3'-(benzyloxy)-2'-hydroxy-[1,1'-binaphthalene]-2-carboxylate (3ak): white solid; $79 \%$ yield, $91 \%$ ee; ${ }^{1} \mathrm{H}-\mathrm{NMR}\left(300 \mathrm{MHz}, \mathrm{CDCl}_{3}\right): \delta=8.16(\mathrm{~d}, 1 \mathrm{H}, J=8.7 \mathrm{~Hz}), 8.01$ $(\mathrm{d}, 1 \mathrm{H}, J=8.7 \mathrm{~Hz}), 7.95(\mathrm{~d}, 1 \mathrm{H}, J=8.1 \mathrm{~Hz}), 7.10(\mathrm{~d}, 1 \mathrm{H}, J=8.1 \mathrm{~Hz}), 7.54\left(\mathrm{dt}, J_{1}=1.2 \mathrm{~Hz}, J_{2}=8.1 \mathrm{~Hz}\right)$, 7.15-7.45 (m, 19H), 7.03-7.08 (m, 1H), $6.94(\mathrm{~d}, 1 \mathrm{H}, J=8.4 \mathrm{~Hz}), 5.86(\mathrm{~s}, 1 \mathrm{H}), 5.18-5.27(\mathrm{~m}, 2 \mathrm{H}), 5.10$ (quint, $1 \mathrm{H}, J=5.4 \mathrm{~Hz}), 4.30-4.39(\mathrm{~m}, 2 \mathrm{H}), 4.17-4.27(\mathrm{~m}, 2 \mathrm{H}), 3.11-3.29(\mathrm{~m}, 3 \mathrm{H}), 2.94\left(\mathrm{dd}, J_{1}=5.7 \mathrm{~Hz}\right.$, $\left.J_{2}=10.5 \mathrm{~Hz}\right) .{ }^{13} \mathrm{C}$ NMR $\left(75 \mathrm{MHz}, \mathrm{CDCl}_{3}, \mathrm{TMS}\right): \delta=166.9,146.1,142.9,138.1,135.9,135.4,135.2$, $132.6,129.7,129.3,128.8,128.5,128.3,128.2,128.1,127.9,127.7,127.5,127.4,126.8,126.7,126.4$, 124.7, 124.6, 123.9, 119.0, 106.8, 73.0, 72.9, 71.6, 71.0, 68.4, 68.1; HPLC (Daicel CHIRALCEL IC column, hexane $/ i \mathrm{PrOH}=70: 30$ at $0.5 \mathrm{~mL} / \mathrm{min}, \lambda=254 \mathrm{~nm}): \mathrm{t}_{\text {major }}=16.80 \mathrm{~min}, \mathrm{t}_{\text {minor }}=19.98 \mathrm{~min}$, ee $=$ $91 \% ;[\alpha]_{\mathrm{D}}^{21.7}$ (major) $=-21.6\left(\mathrm{c}=1.00, \mathrm{CHCl}_{3}\right)$.

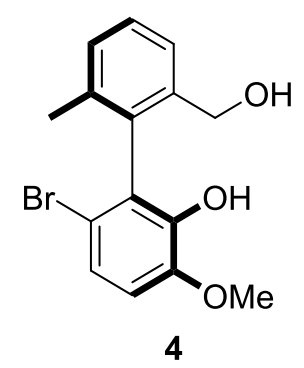

(R)-6-Bromo-2'-(hydroxymethyl)-3-methoxy-6'-methyl-[1,1'-biphenyl]-2-ol (4): white solid; $93 \%$ yield, 94\% ee; ${ }^{1} \mathrm{H}-\mathrm{NMR}\left(300 \mathrm{MHz}, \mathrm{CDCl}_{3}\right): \delta=7.42(\mathrm{~d}, 1 \mathrm{H}, J=7.2 \mathrm{~Hz}), 7.37(\mathrm{t}, 1 \mathrm{H}, J=7.5 \mathrm{~Hz}), 7.28$ $(\mathrm{d}, 1 \mathrm{H}, J=7.5 \mathrm{~Hz}), 7.23(\mathrm{~d}, 1 \mathrm{H}, J=8.7 \mathrm{~Hz}), 6.80(\mathrm{~d}, 1 \mathrm{H}, J=8.7 \mathrm{~Hz}), 5.76(\mathrm{~s}, 1 \mathrm{H}), 4.28-4.38(\mathrm{~m}, 2 \mathrm{H})$, $3.93(\mathrm{~s}, 3 \mathrm{H}), 2.03(\mathrm{~s}, 3 \mathrm{H}) .{ }^{13} \mathrm{C}$ NMR $\left(75 \mathrm{MHz}, \mathrm{CDCl}_{3}, \mathrm{TMS}\right): \delta=146.2,143.5,138.9,137.1,134.9$, 129.6, 128.7, 126.1, 123.6, 115.4, 111.2, 64.0, 56.1, 19.7; HPLC (Daicel CHIRALCEL AD column, hexane $/ \mathrm{PrOH}=85: 15$ at $0.5 \mathrm{~mL} / \mathrm{min}, \lambda=210 \mathrm{~nm}): \mathrm{t}_{\text {major }}=18.62 \mathrm{~min}, \mathrm{t}_{\text {minor }}=26.01 \mathrm{~min}$, ee $=94 \%$; $[\alpha]_{\mathrm{D}}^{25.3}$ (major) $=+17.7\left(\mathrm{c}=1.00, \mathrm{CHCl}_{3}\right)$.<smiles>Cc1cc(C)c(-c2c(CO)ccc3ccccc23)c(O)c1</smiles>

(S)-2-(2-(Hydroxymethyl)naphthalen-1-yl)-3,5-dimethylphenol (5): white solid; 95\% yield, 90\% ee; ${ }^{1} \mathrm{H}-\mathrm{NMR}\left(300 \mathrm{MHz}, \mathrm{CDCl}_{3}\right): \delta=7.87(\mathrm{~d}, 2 \mathrm{H}, J=8.4 \mathrm{~Hz}), 7.65(\mathrm{~d}, 1 \mathrm{H}, J=8.4 \mathrm{~Hz}), 7.46-7.51(\mathrm{~m}, 1 \mathrm{H})$, 7.36-7.38 (m, 2H), $6.78(\mathrm{~s}, 1 \mathrm{H}), 6.70(\mathrm{~s}, 1 \mathrm{H}), 4.46(\mathrm{~s}, 2 \mathrm{H}), 2.36(\mathrm{~s}, 3 \mathrm{H}), 1.77(\mathrm{~s}, 3 \mathrm{H}) .{ }^{13} \mathrm{C} \mathrm{NMR}(75 \mathrm{MHz}$, $\left.\mathrm{CDCl}_{3}, \mathrm{TMS}\right): \delta=153.1,139.2,138.0,137.4,133.4,132.5,131.2,128.9,128.2,126.8,126.4,126.2$, 
125.4, 123.3, 121.0, 114.0, 63.7, 21.3, 19.7; HPLC (Daicel CHIRALCEL AS-H column, hexane/iPrOH $=70: 30$ at $0.5 \mathrm{~mL} / \mathrm{min}, \lambda=254 \mathrm{~nm}): \mathrm{t}_{\text {major }}=8.36 \mathrm{~min}, \mathrm{t}_{\text {minor }}=12.01 \mathrm{~min}$, ee $=90 \% ;[\alpha]_{\mathrm{D}}{ }^{22}$ (major) $=$ $+14.2(\mathrm{c}=0.51, \mathrm{MeOH})$. 
4. Original ${ }^{1} \mathrm{H}$ and ${ }^{13} \mathrm{C}$ NMR Spectra and Chiral HPLC Analysis Trace
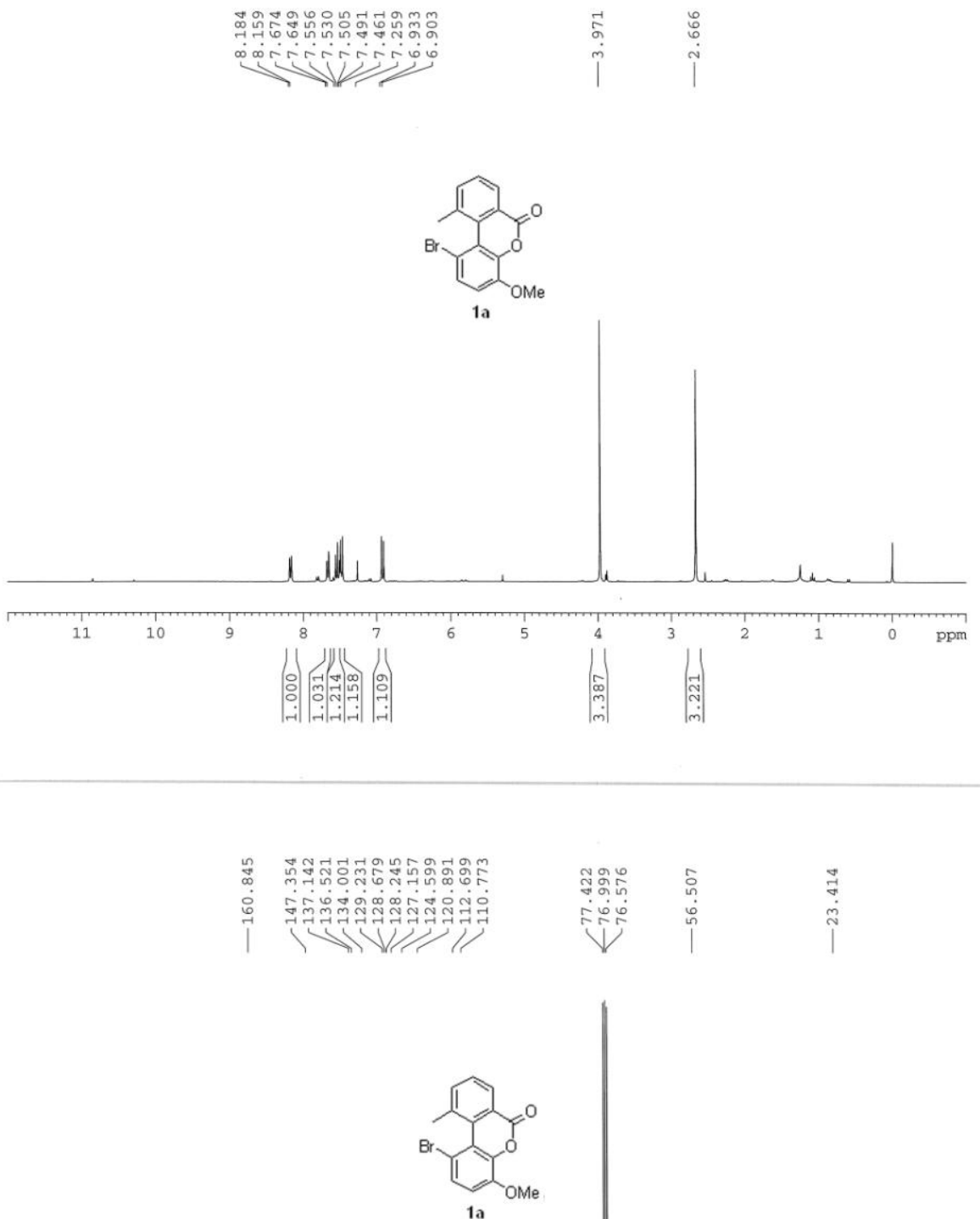

1a

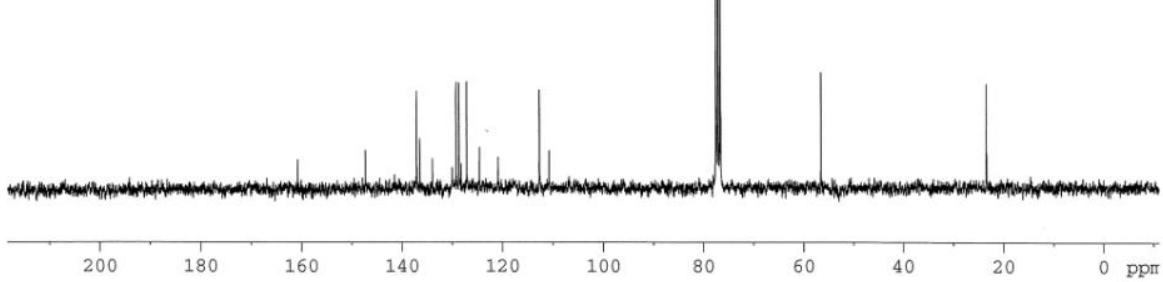




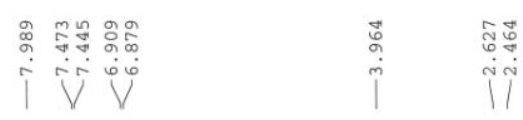
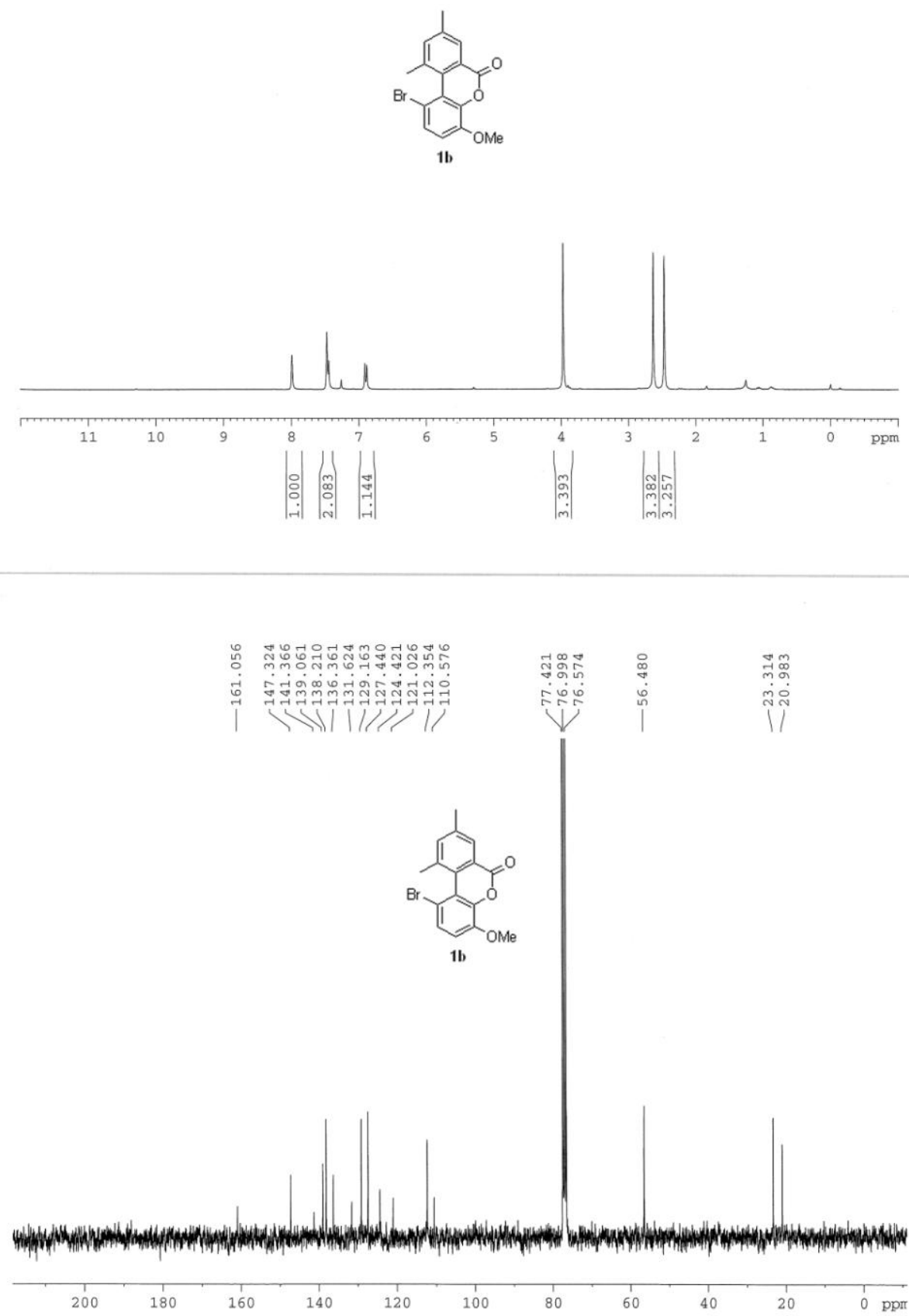

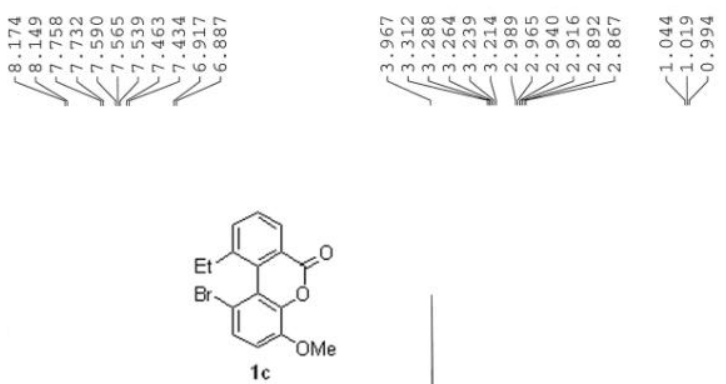

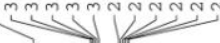

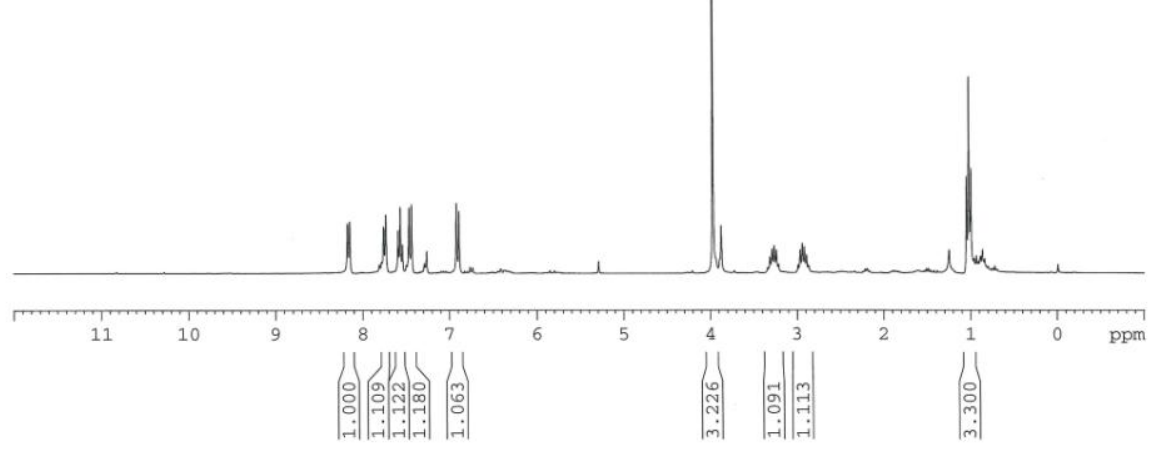

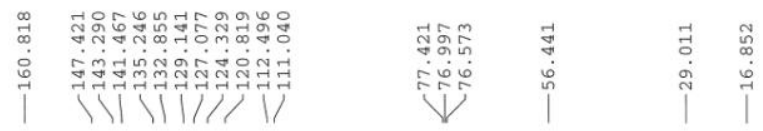
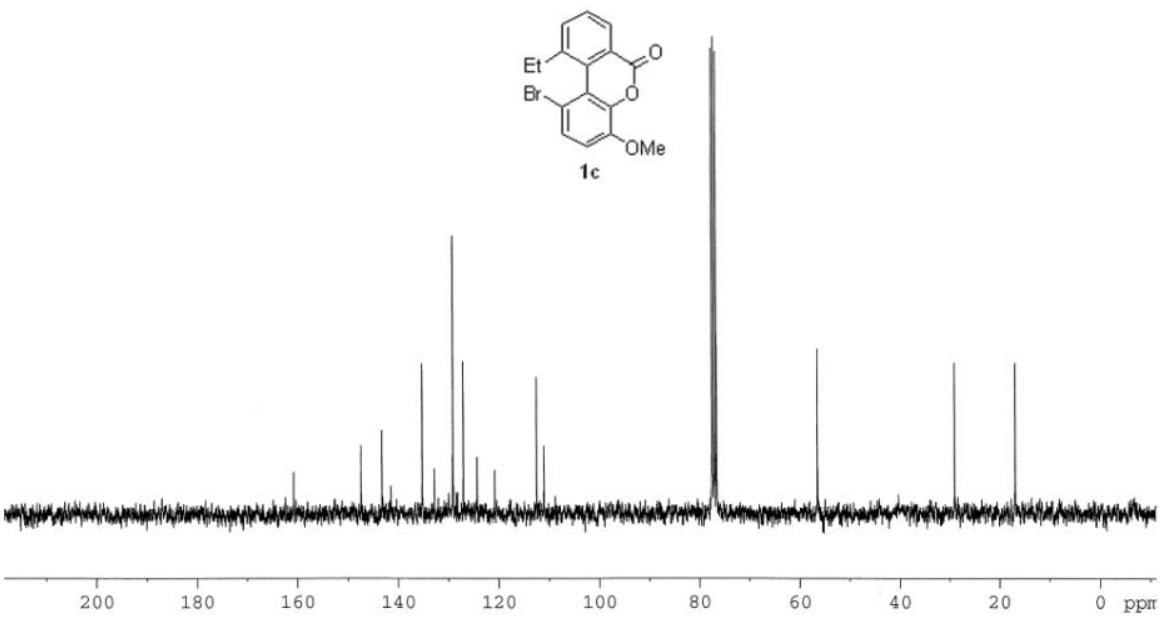

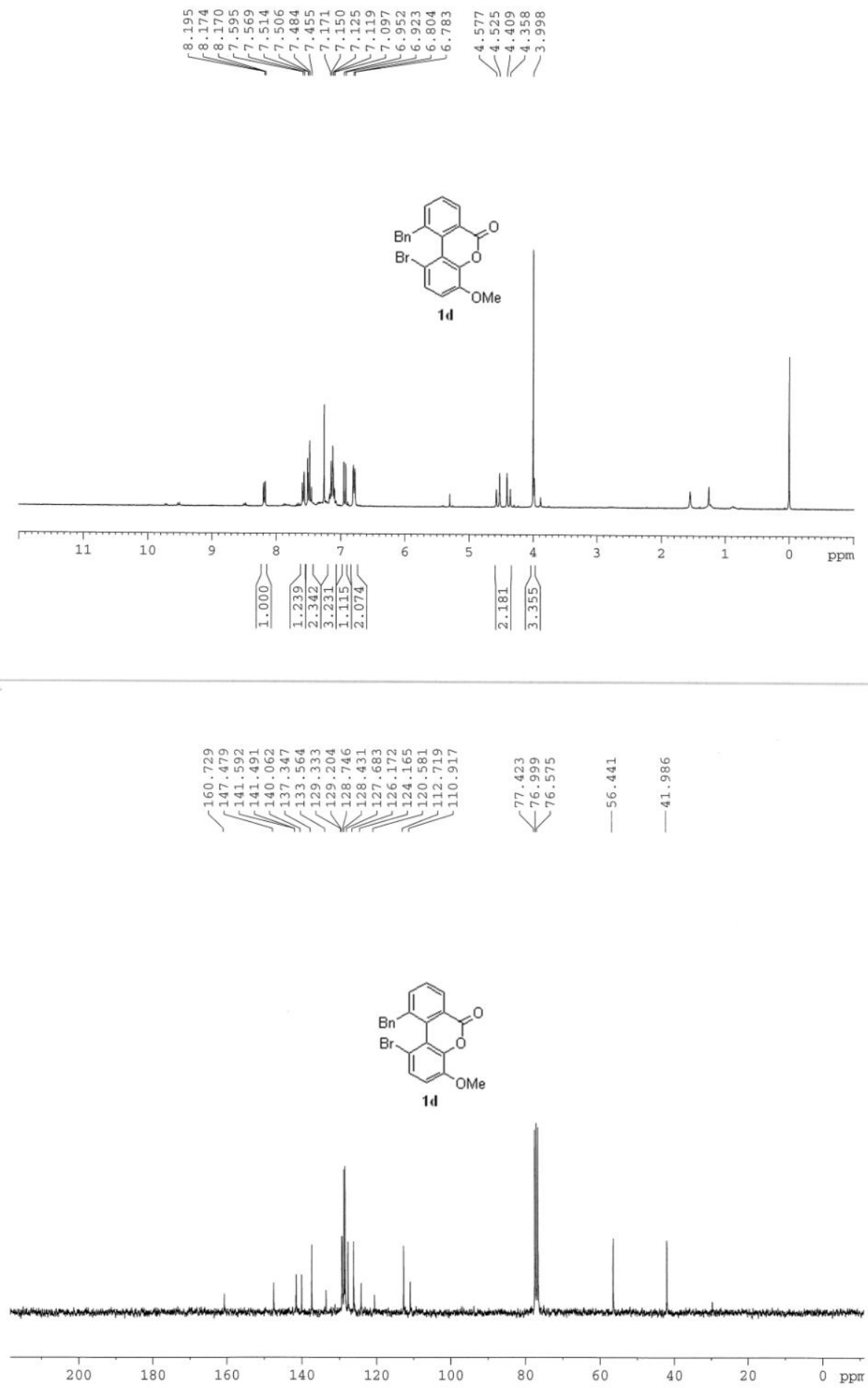


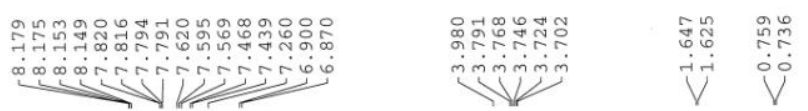
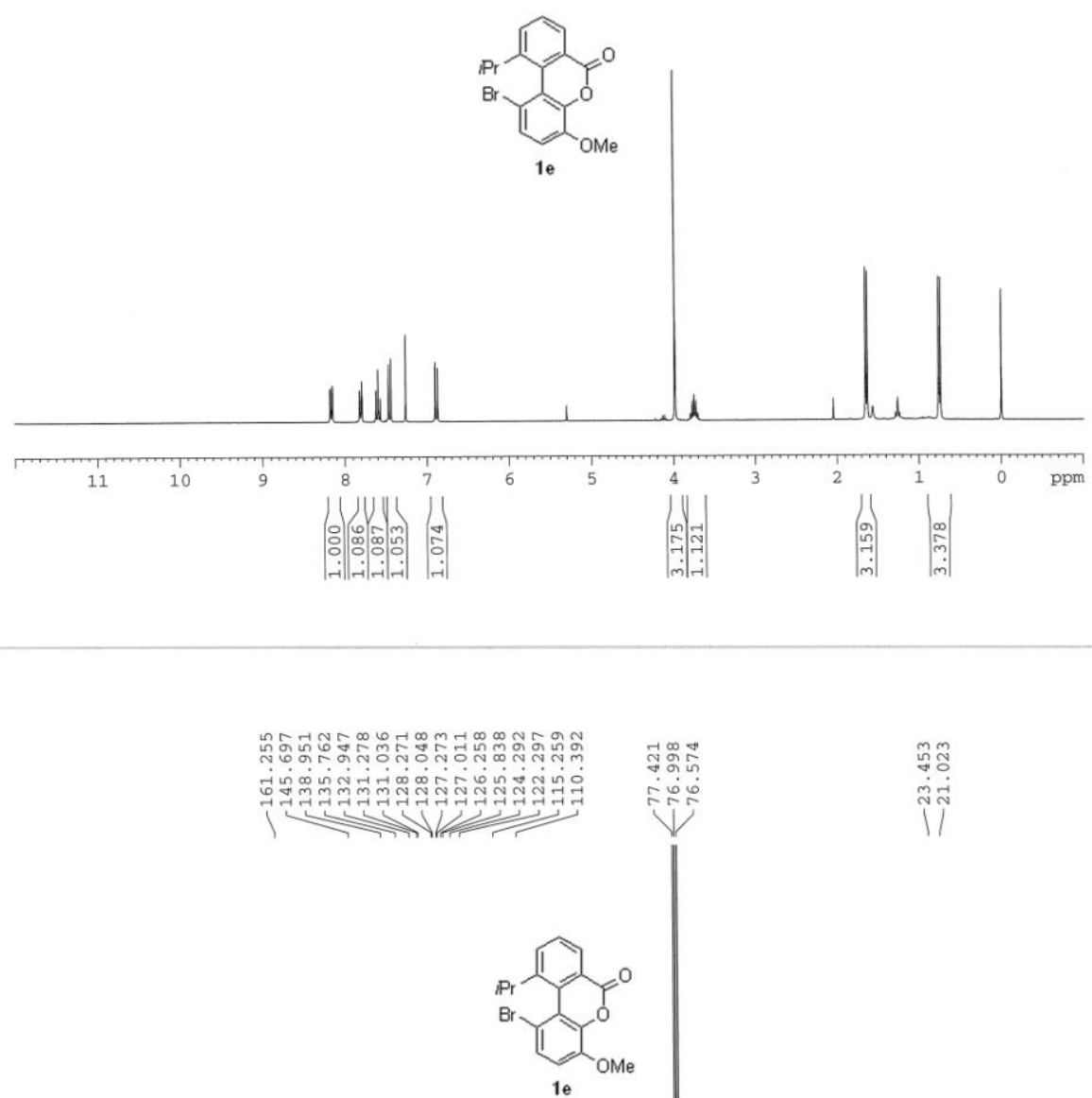

ชุำ

สู่

ㄴํㄴ

\ี

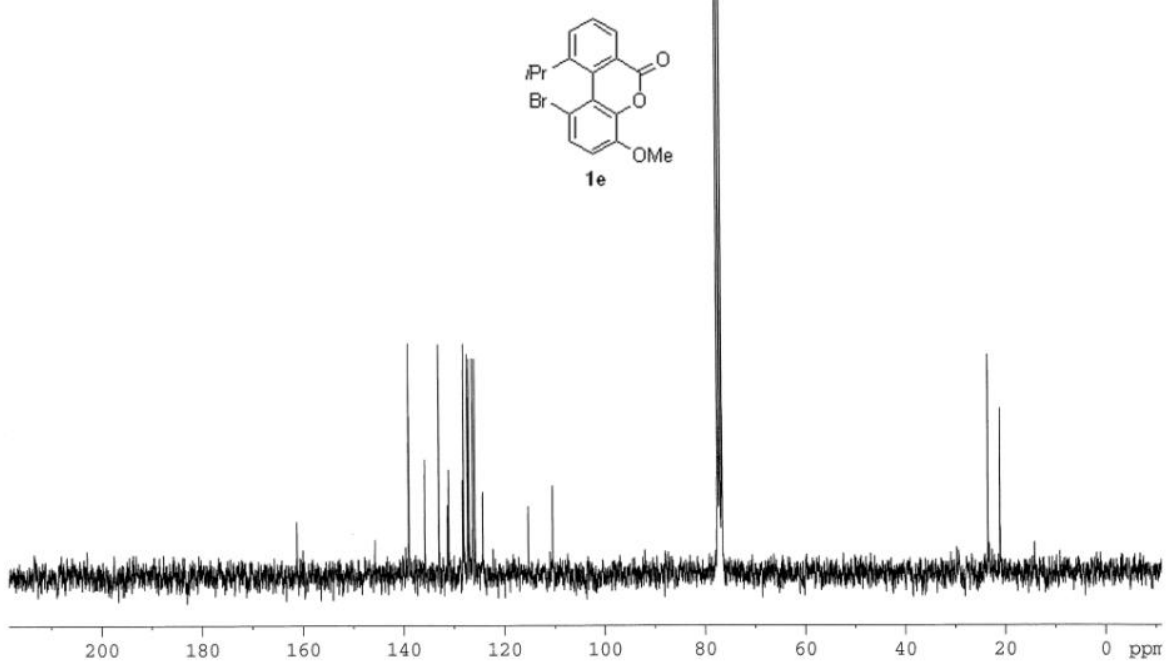



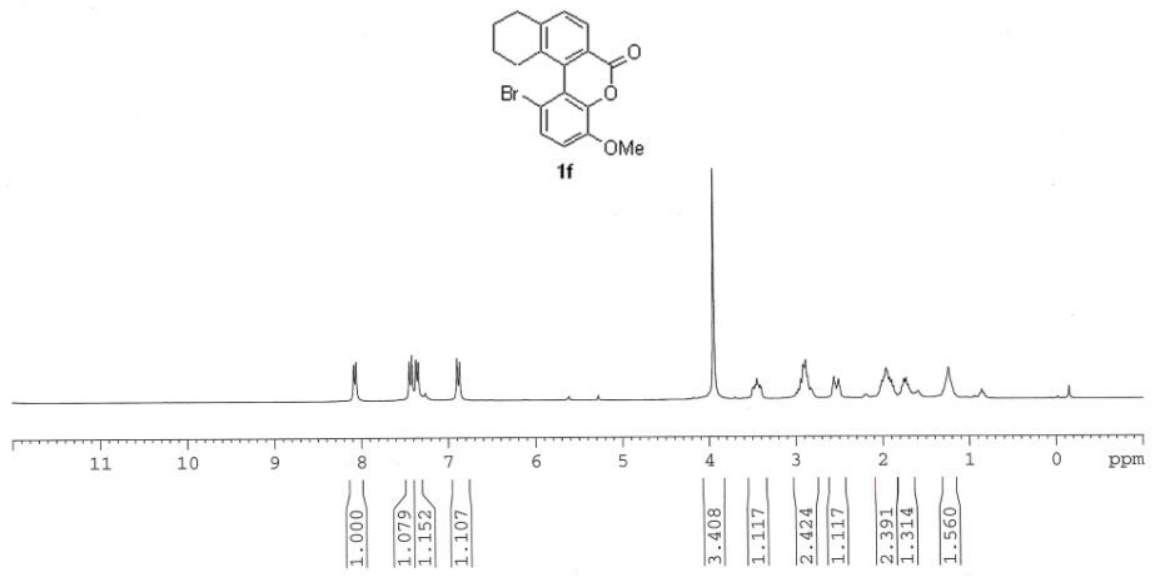

ycg-07-53'-C
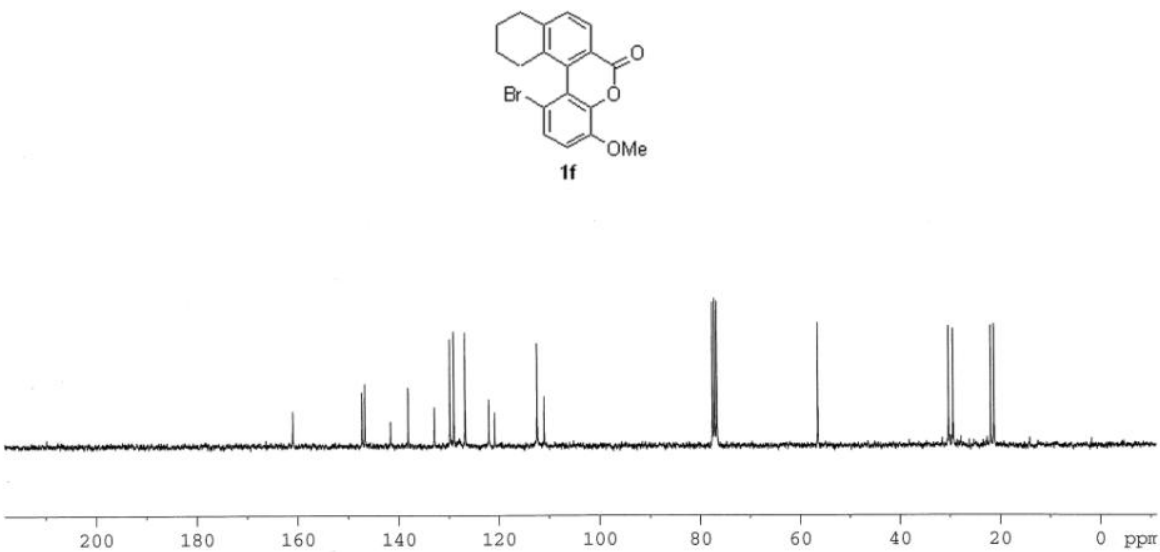


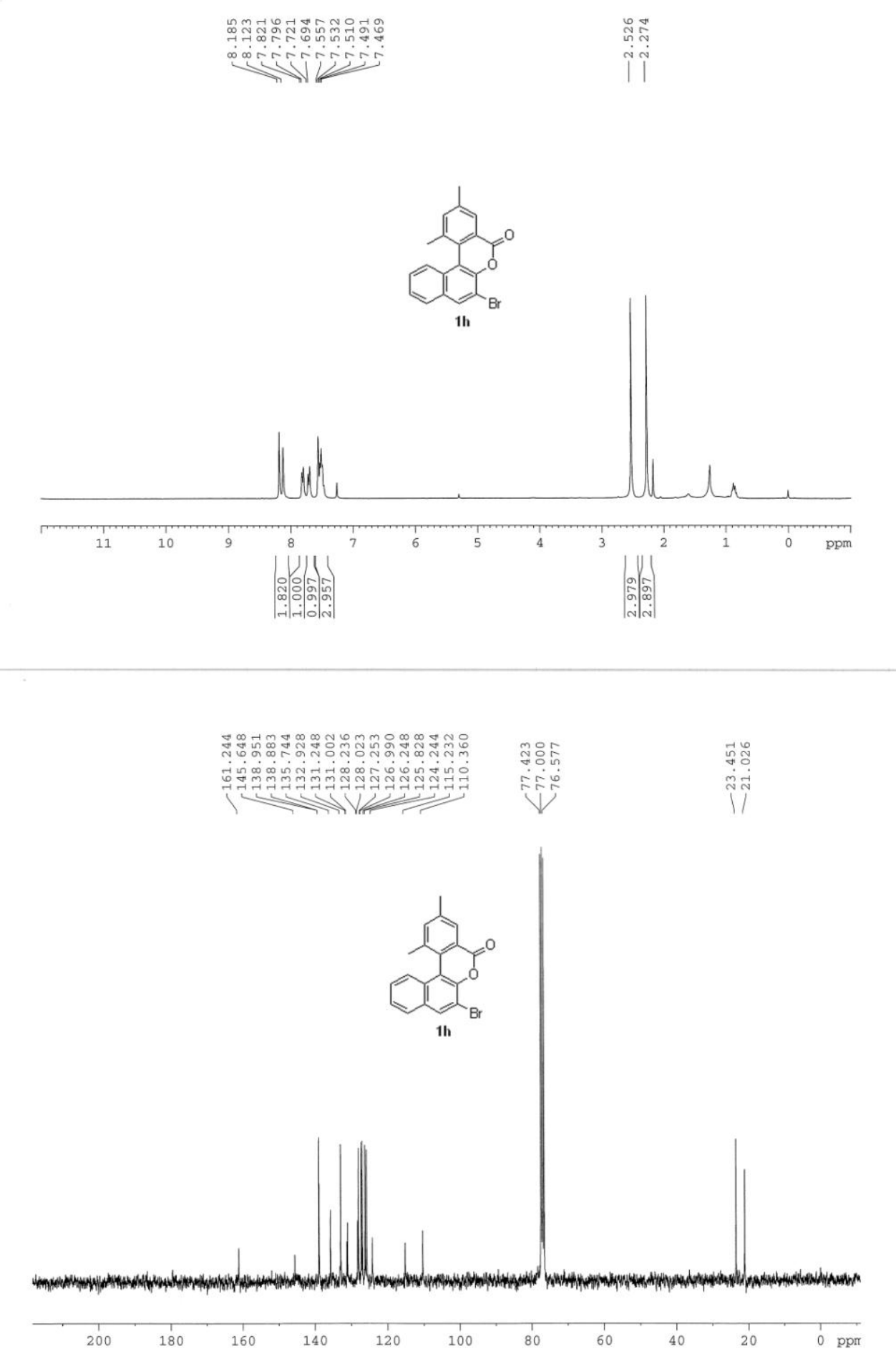




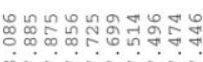

onivivitist

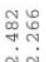
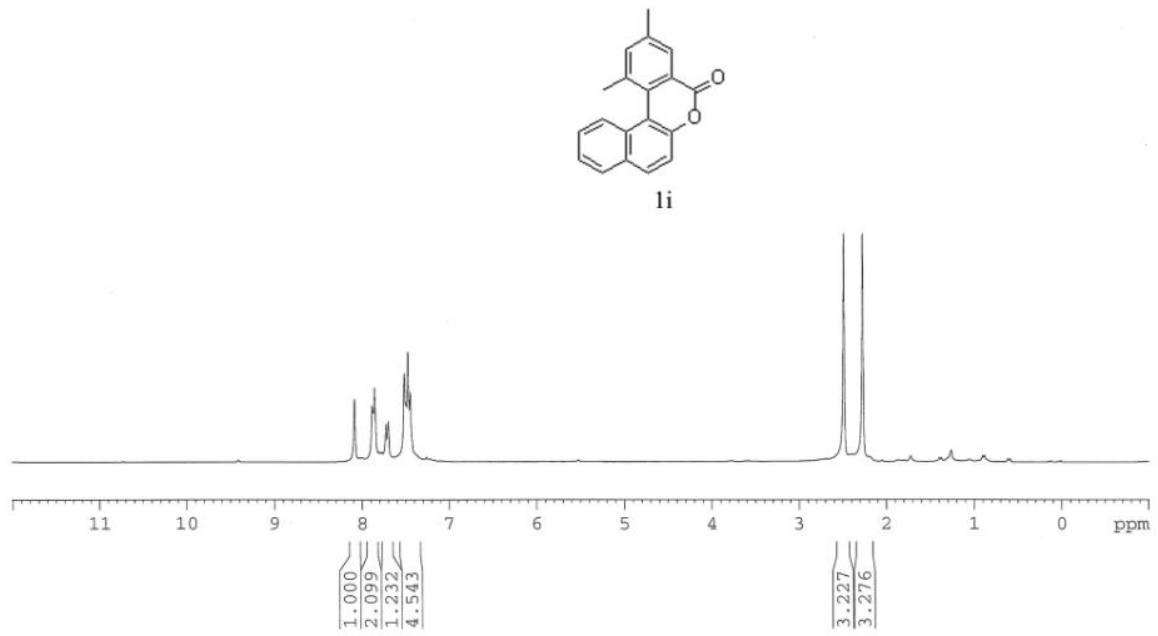

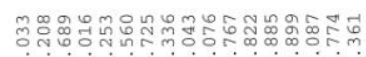

ชंต

(

สู้ ถูกิ์

ㄷํ요

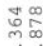

ஸึ่

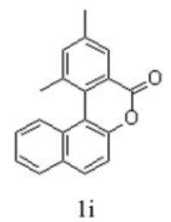

$1 \mathrm{i}$
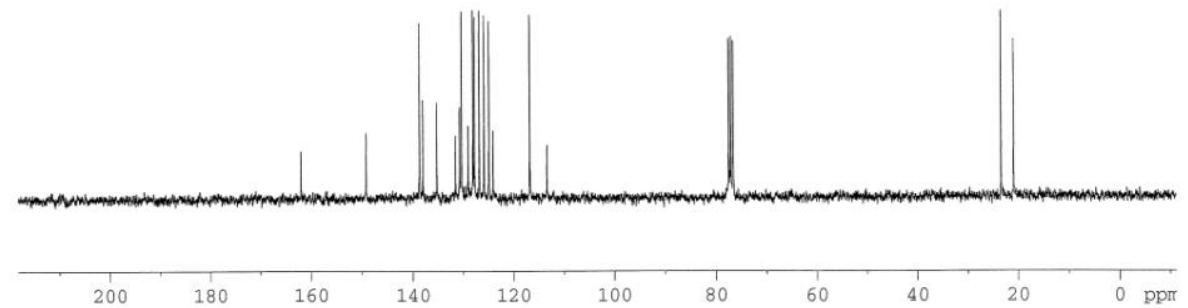


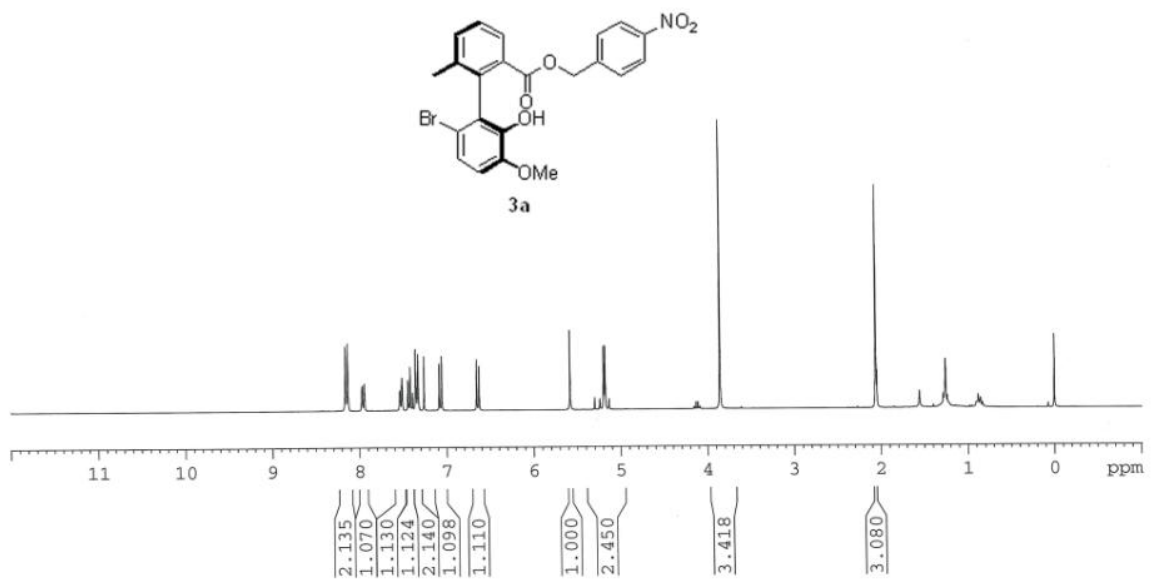

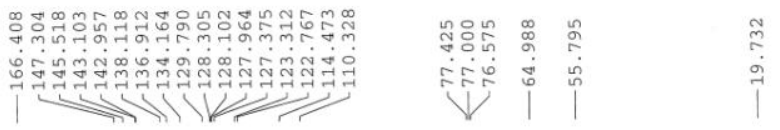
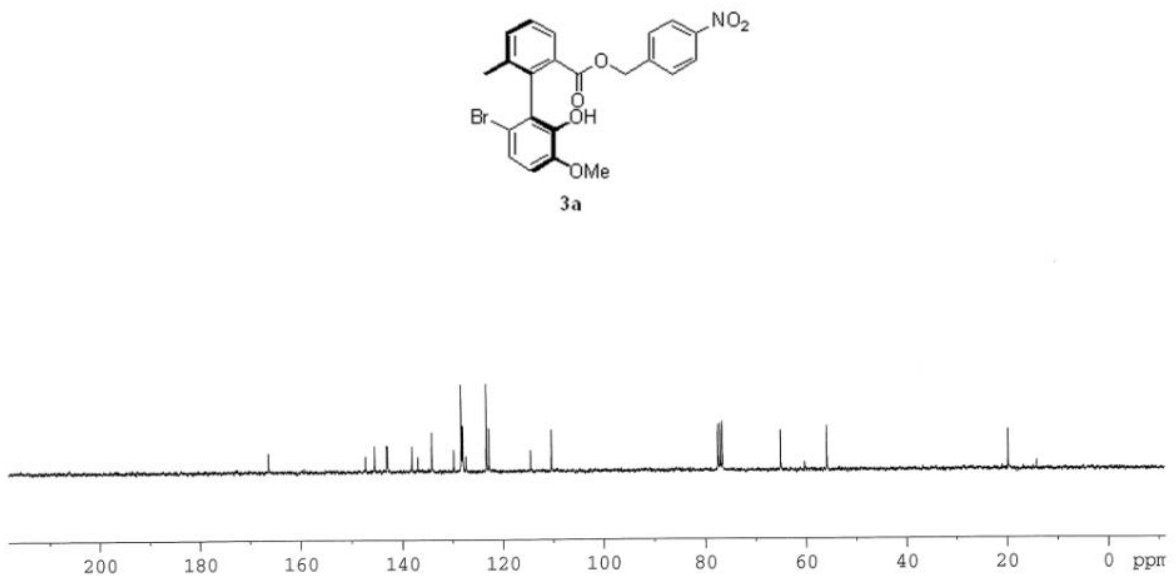

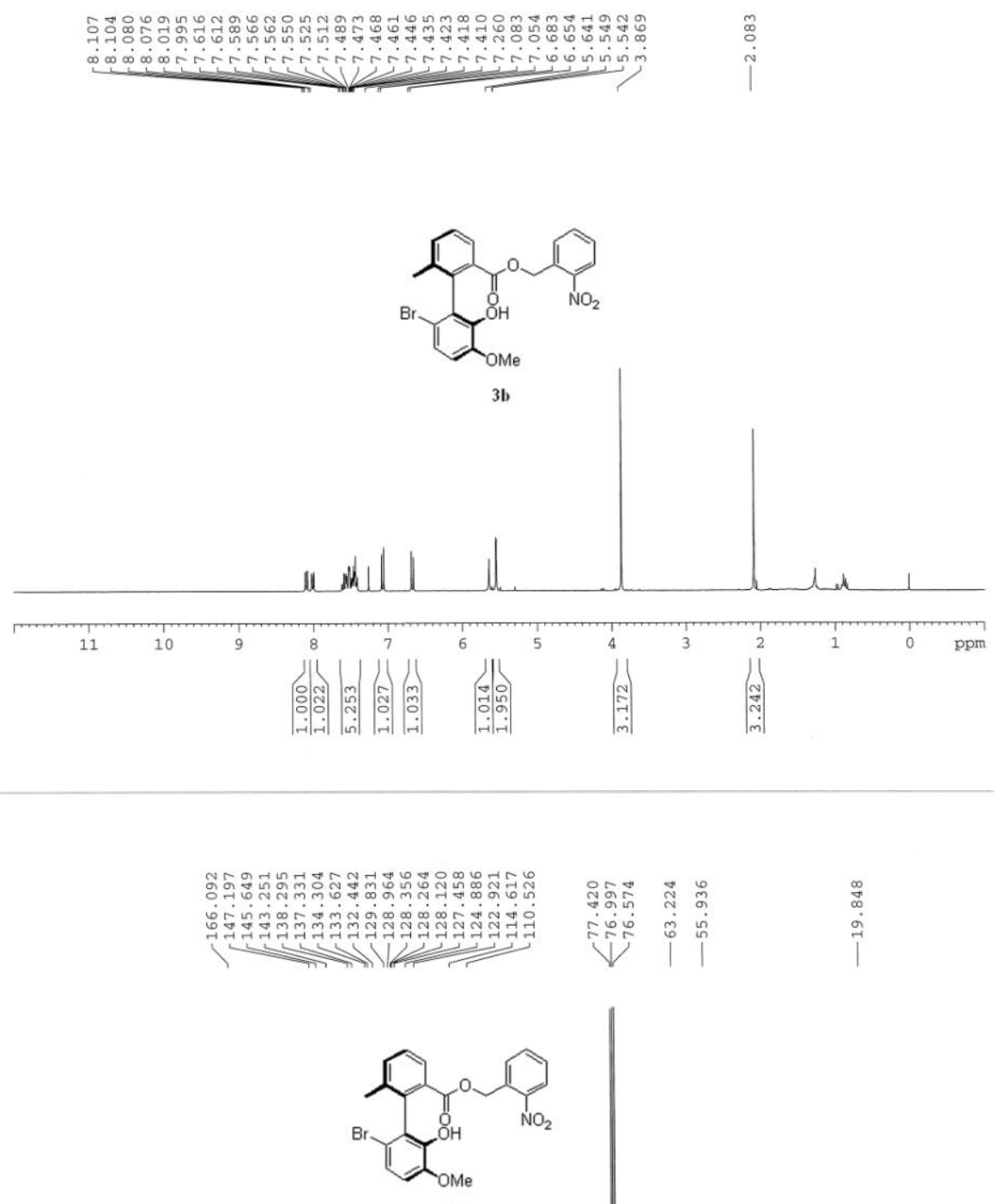

3b

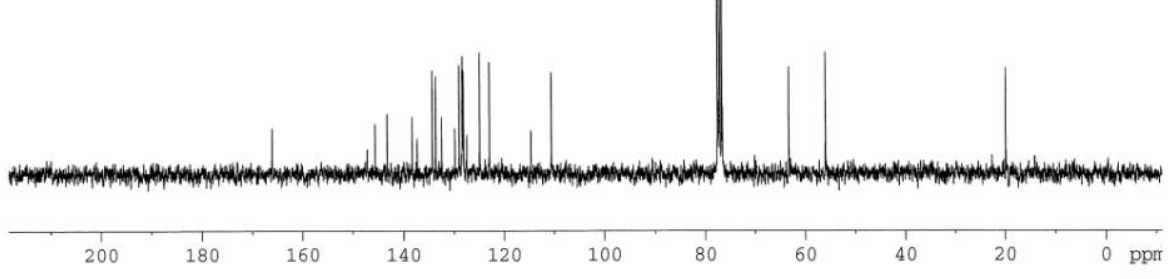



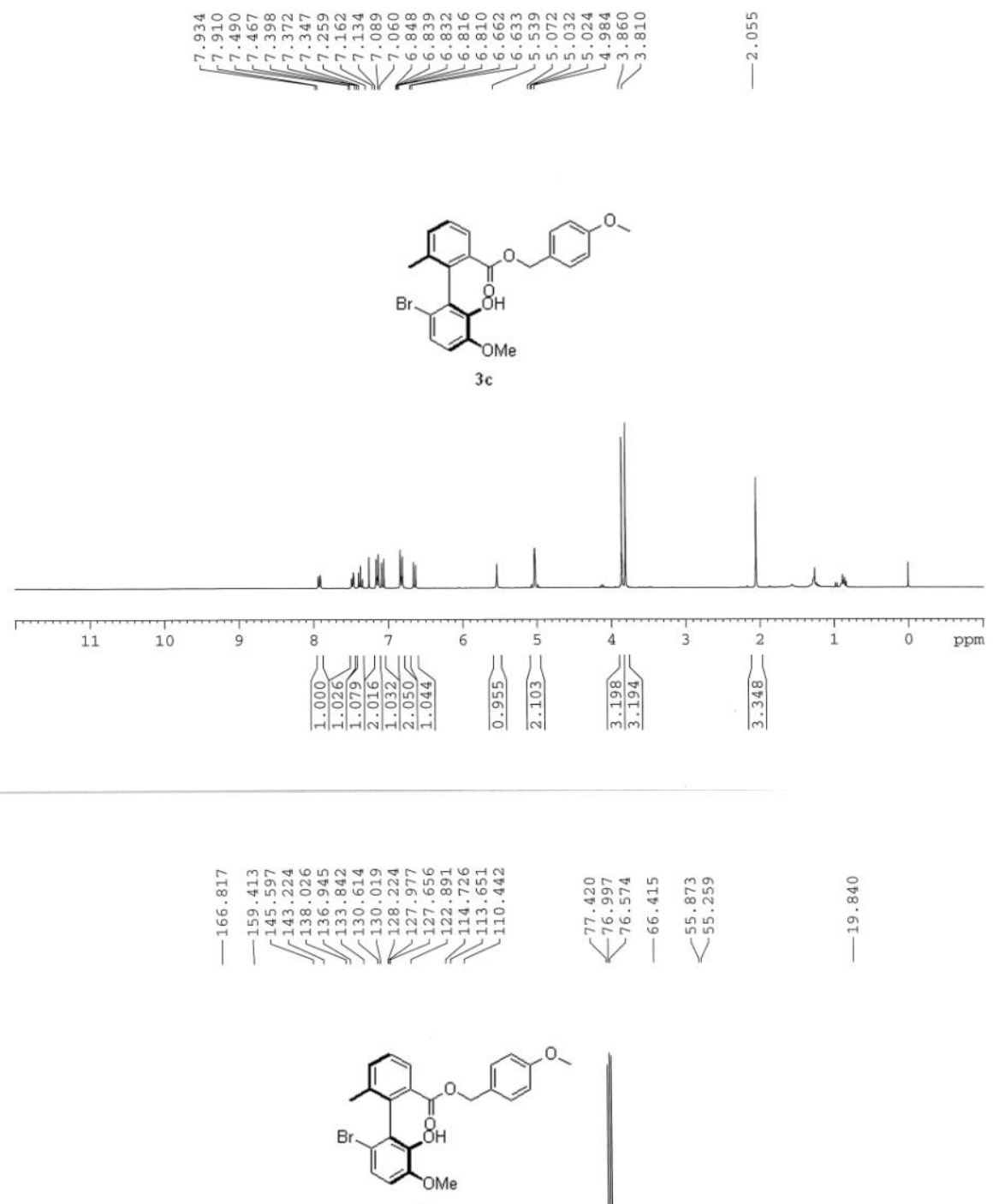

$3 c$

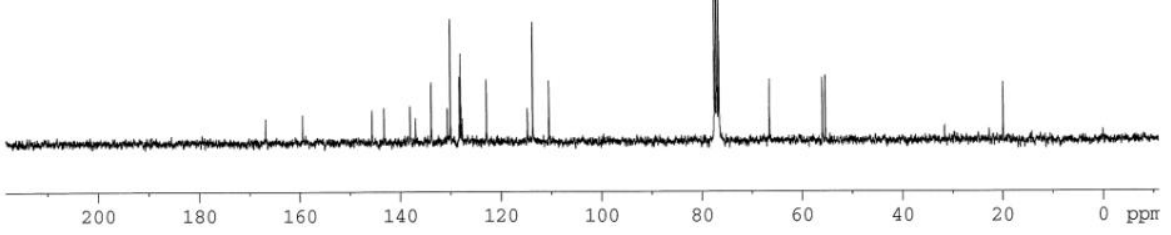




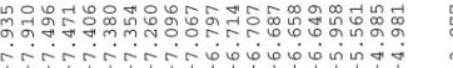
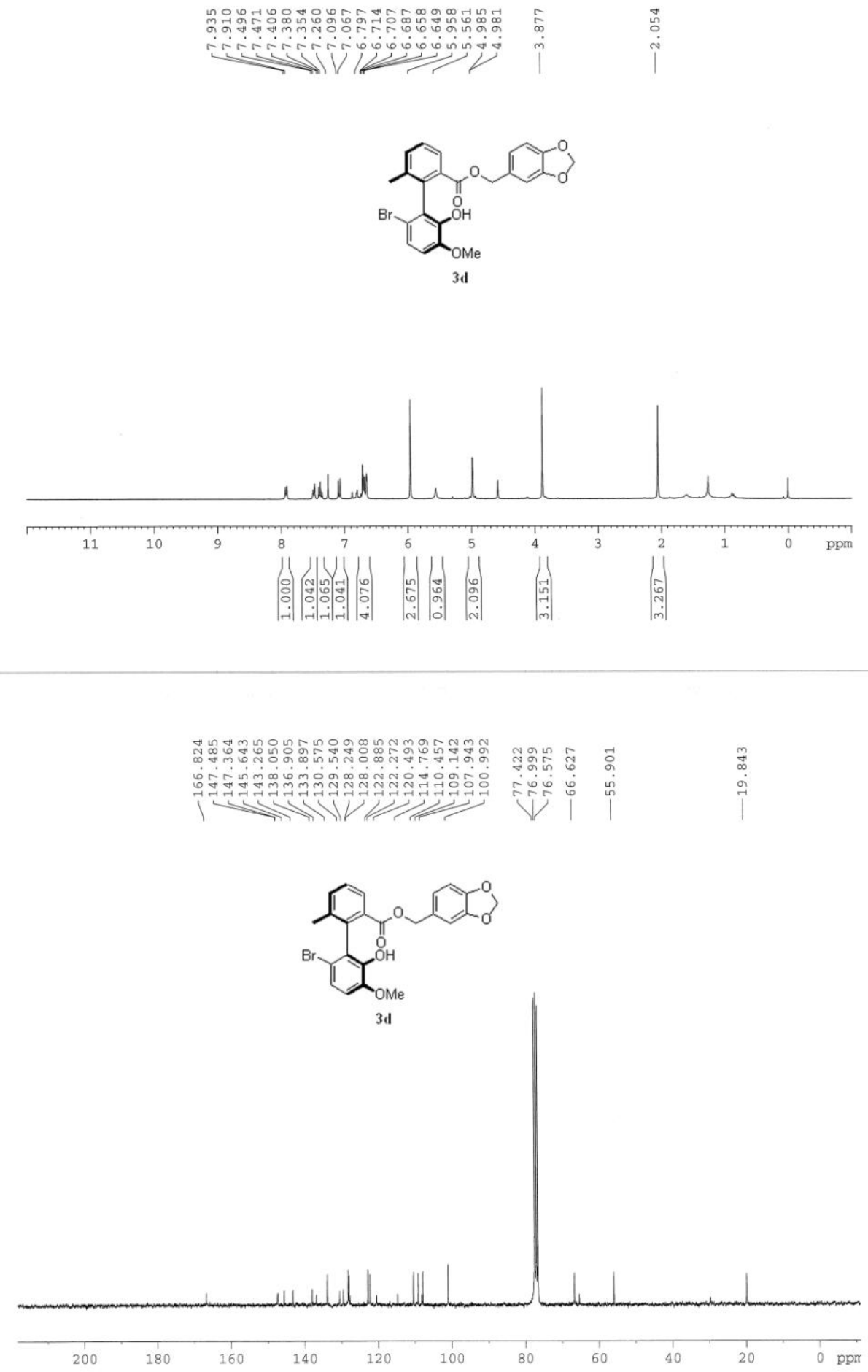

$\stackrel{\infty}{\circ}$ 

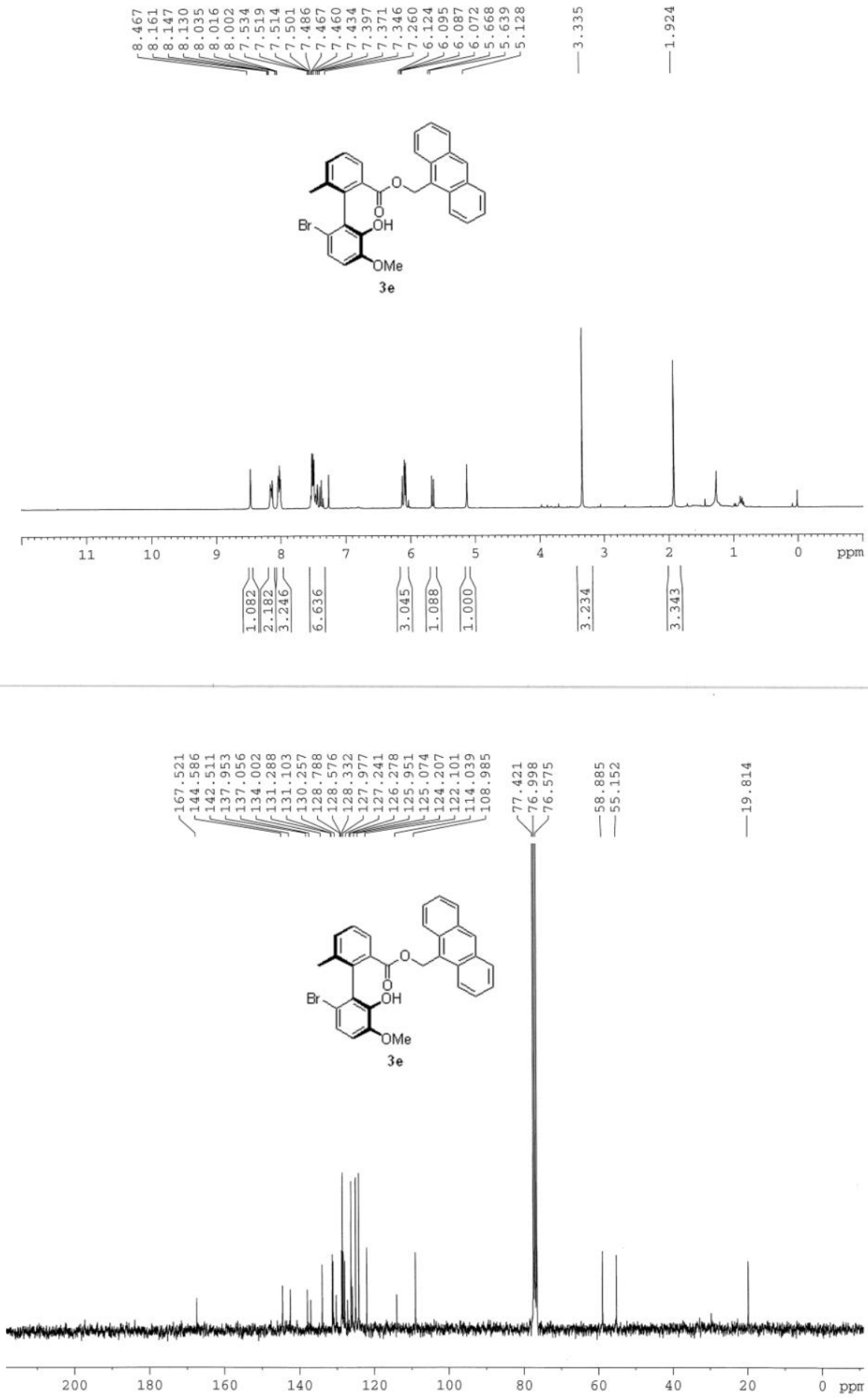


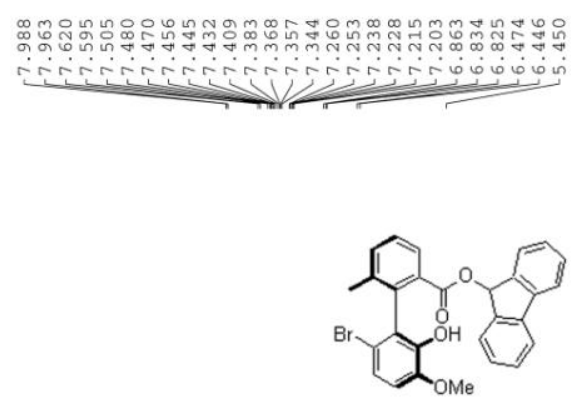

$3 f$
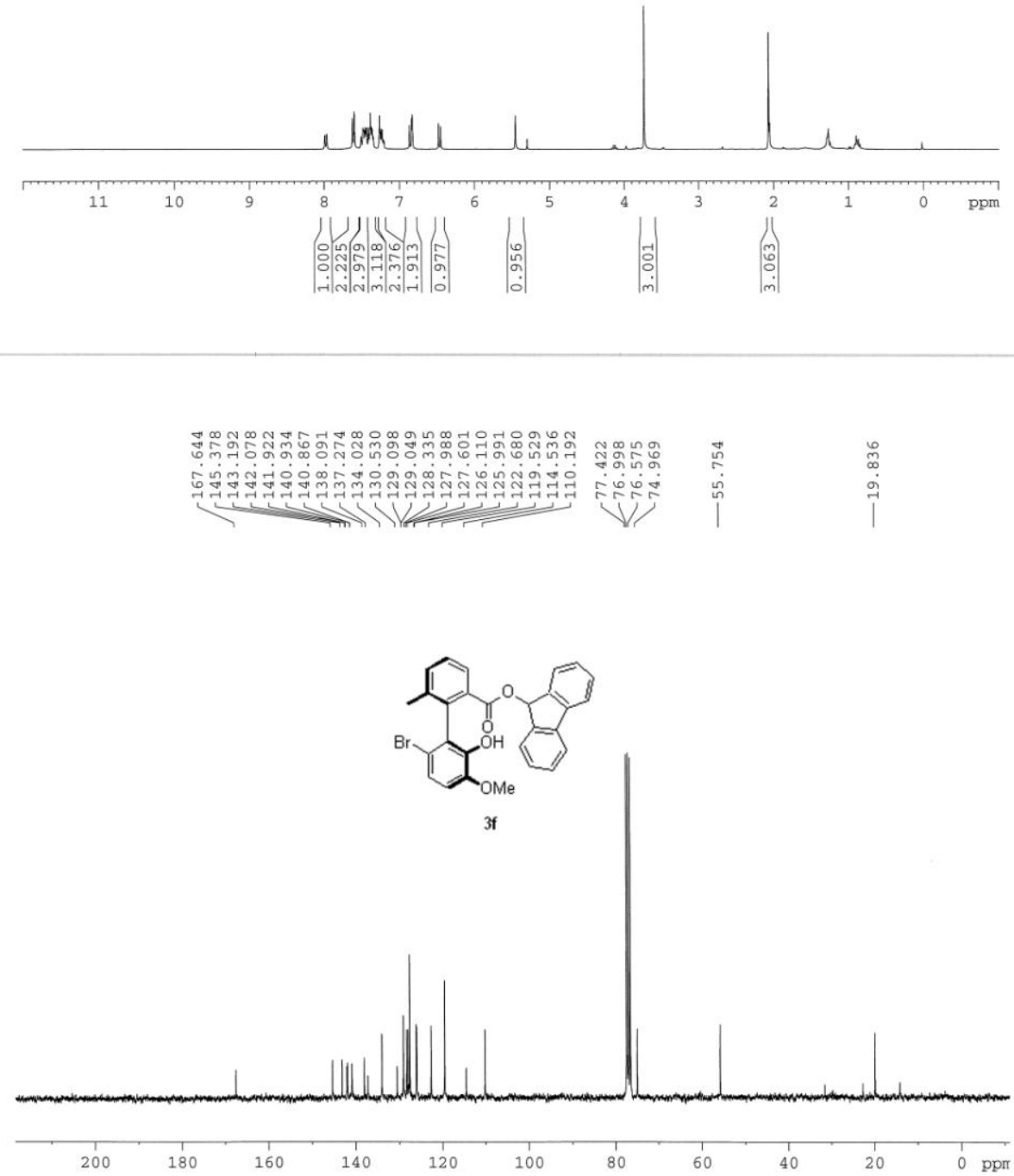

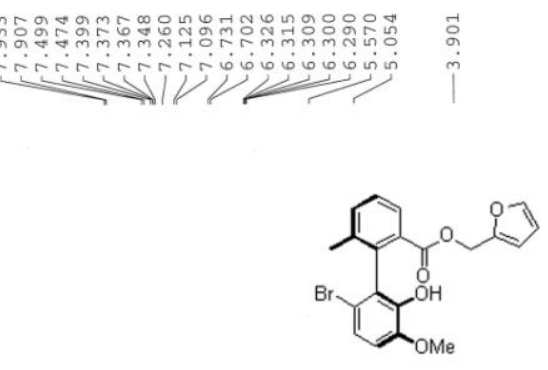

39
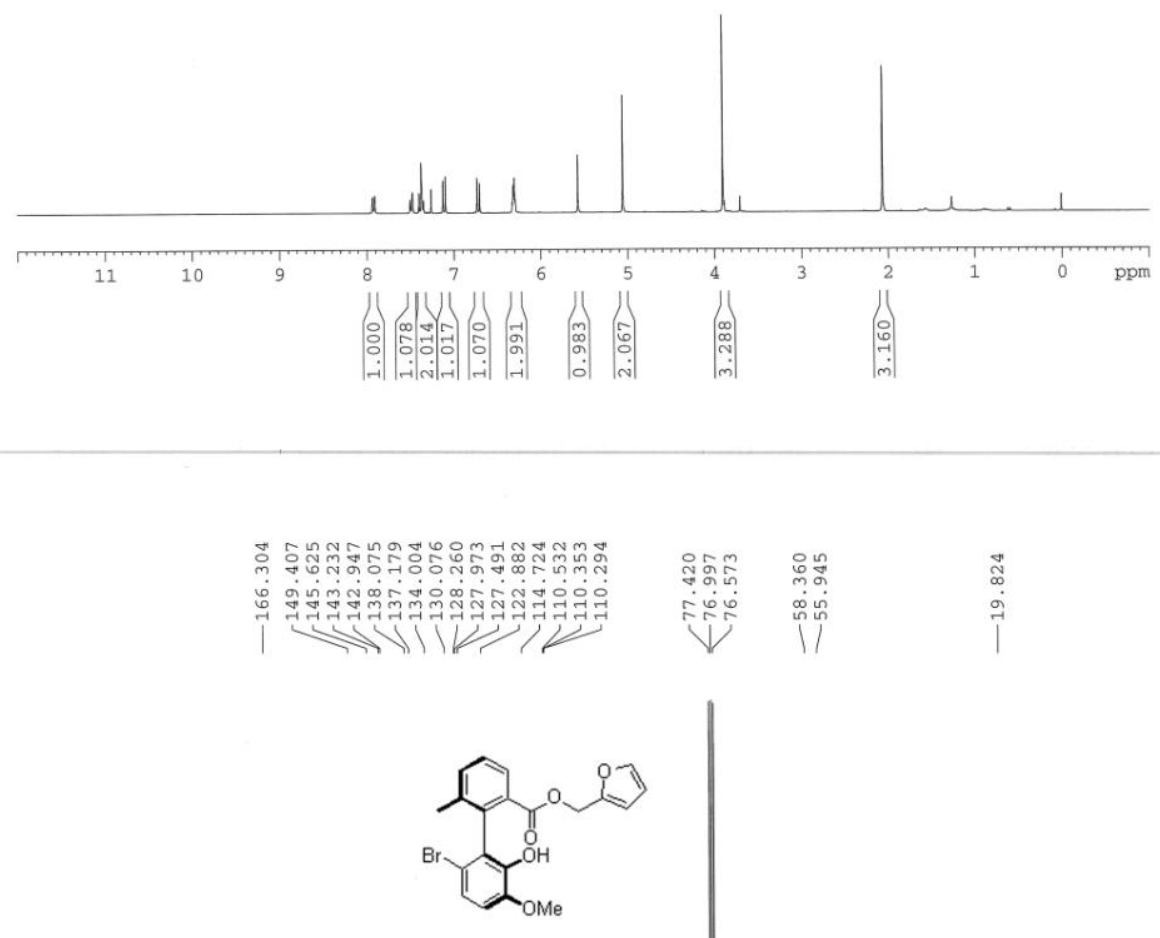

3g

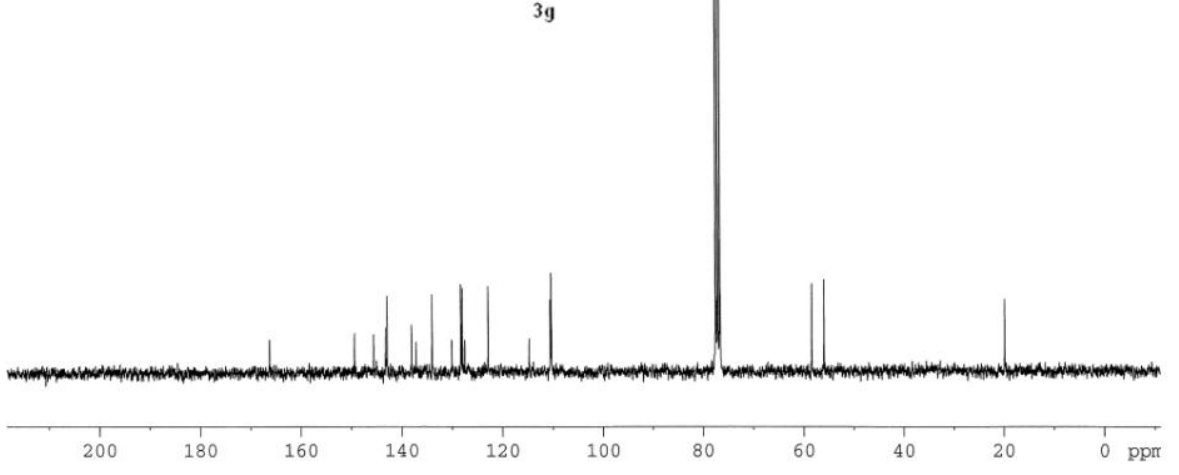



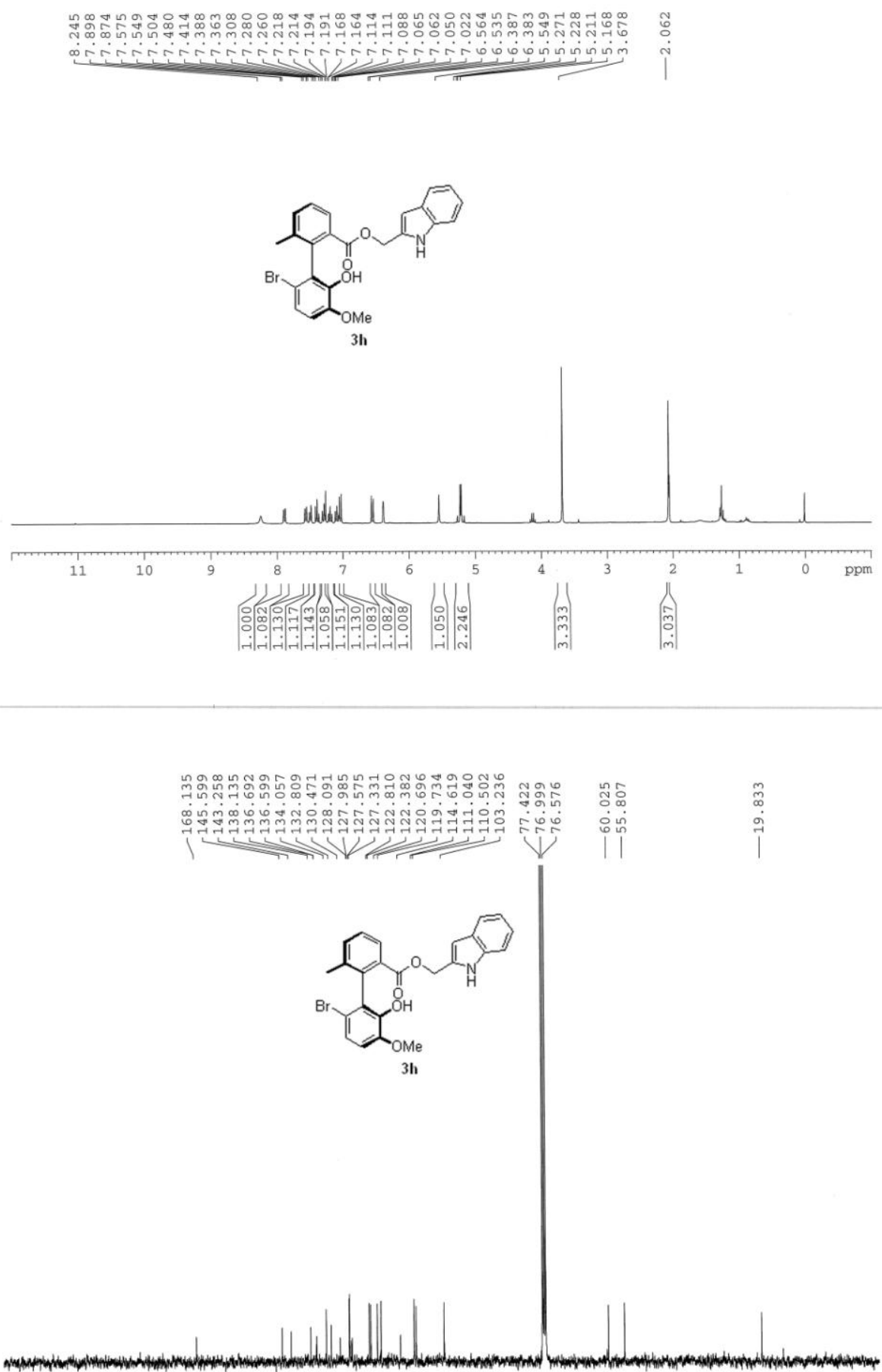

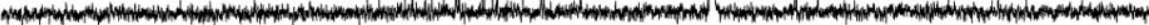



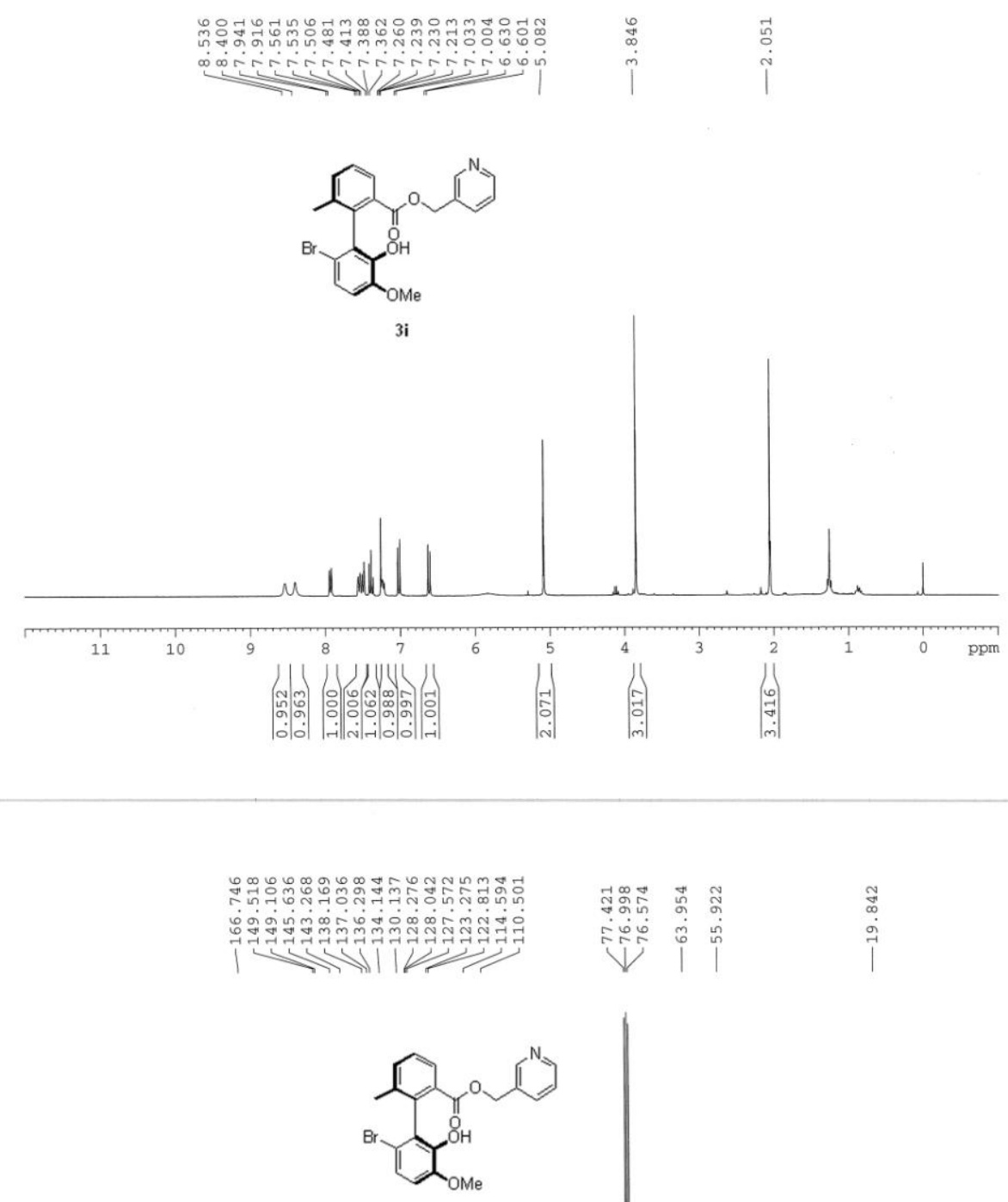

$3 \mathbf{i}$
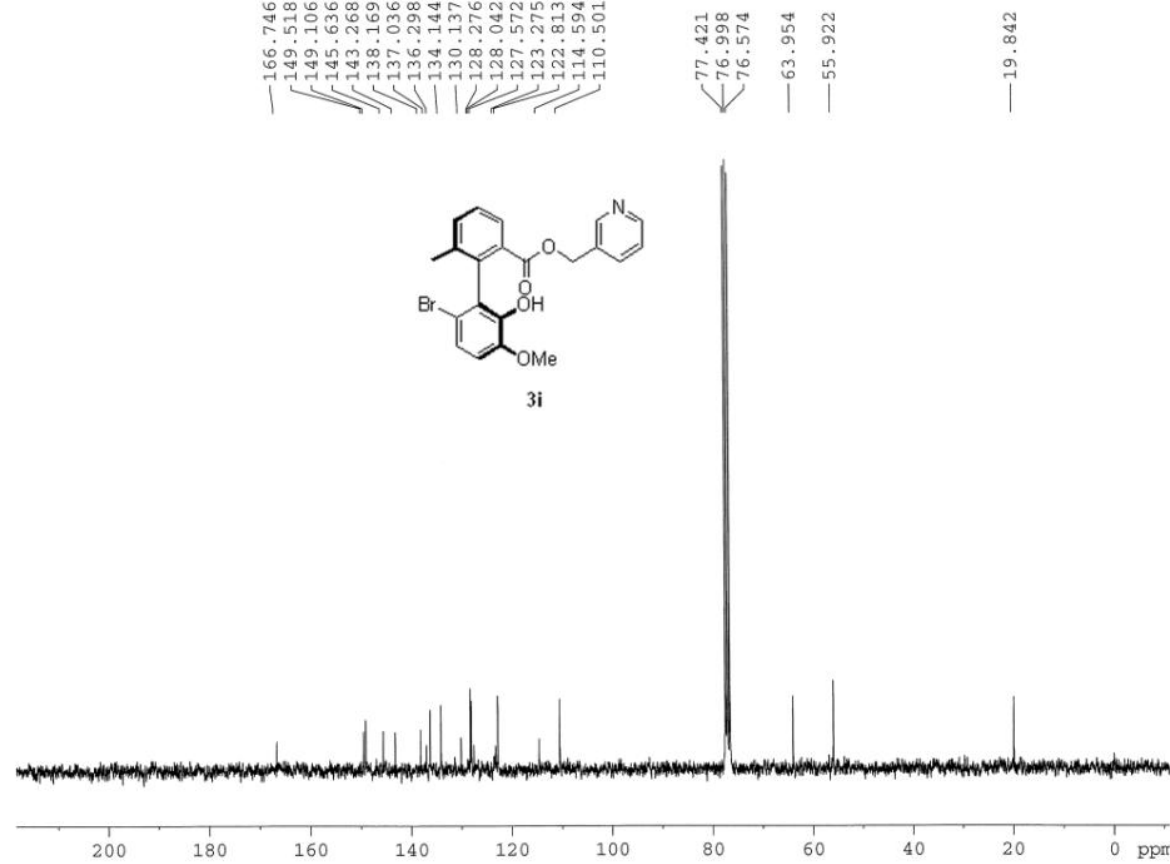


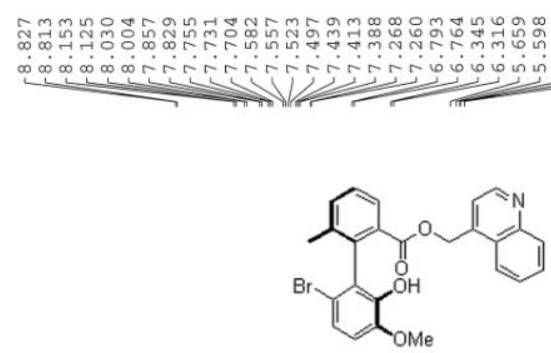

$3 \mathbf{j}$
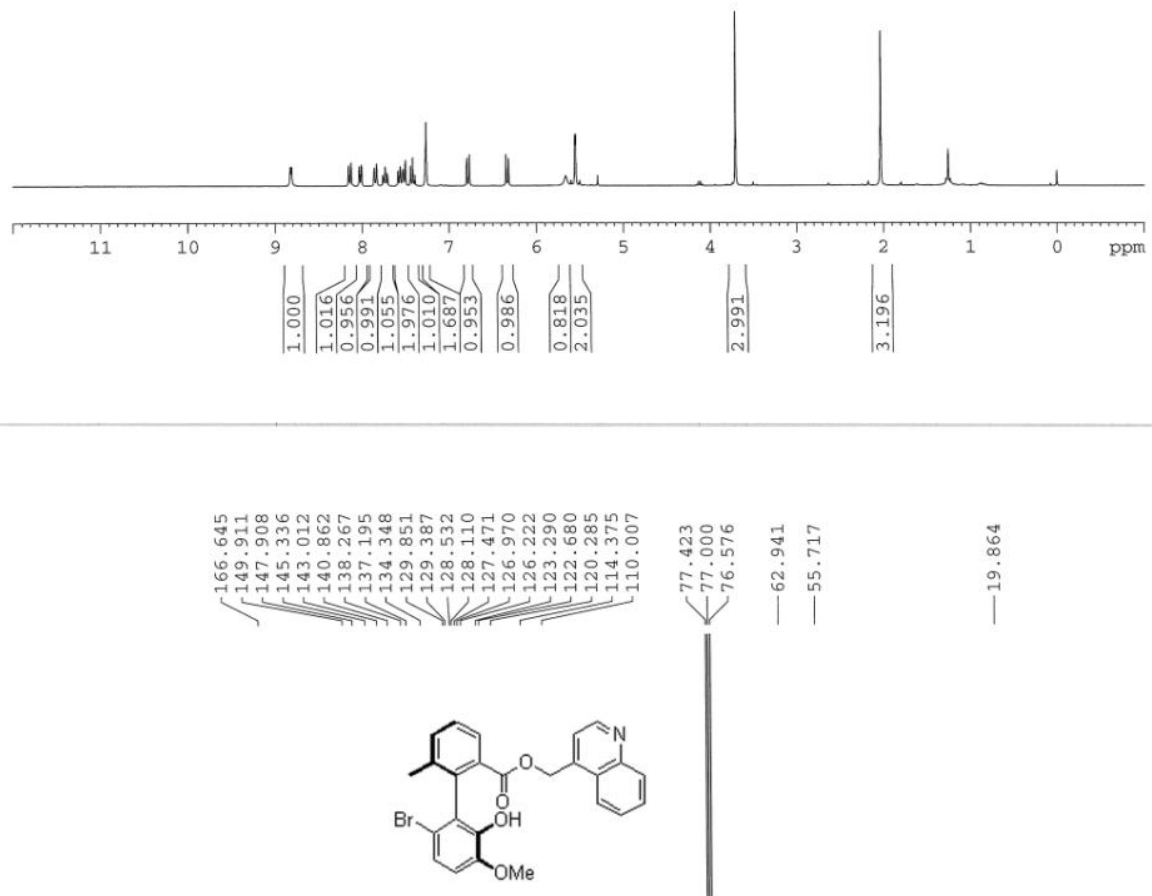

$3 j$

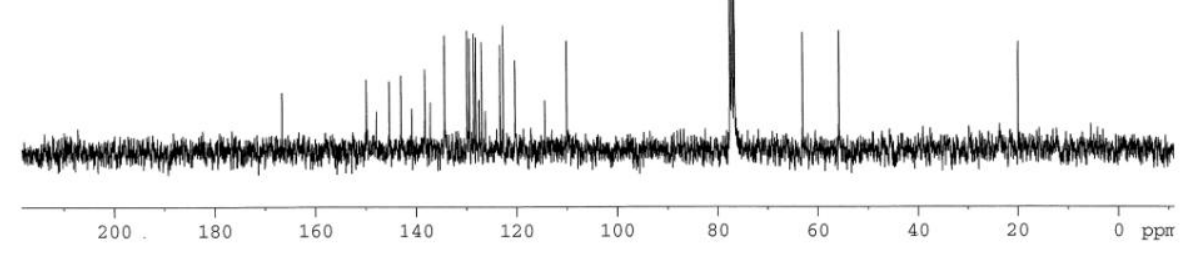



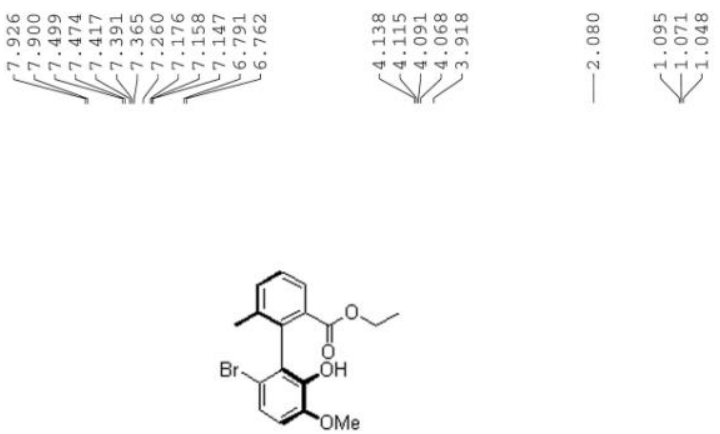

$3 \mathrm{k}$

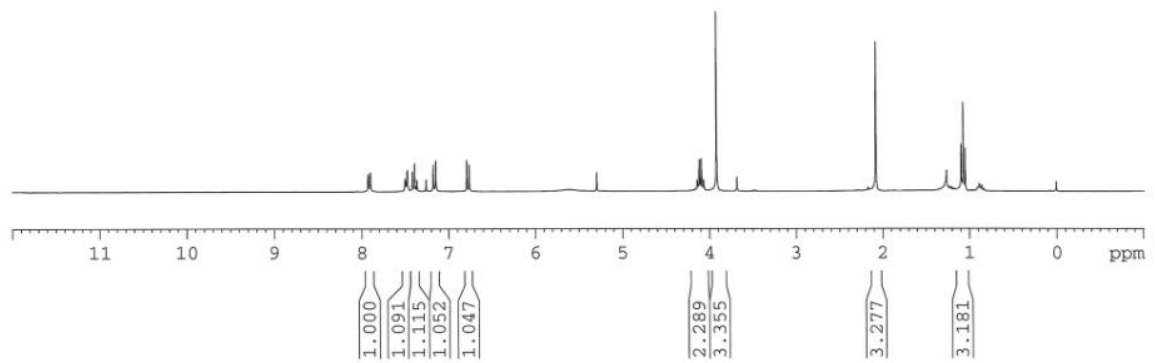

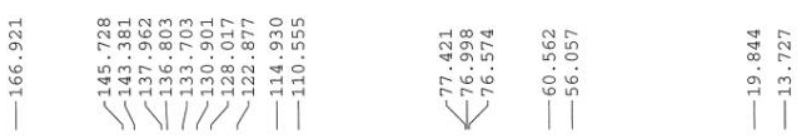

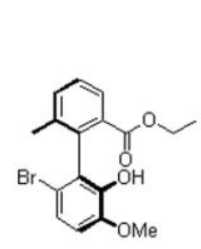

$3 k$

4.

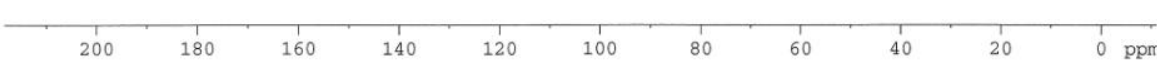



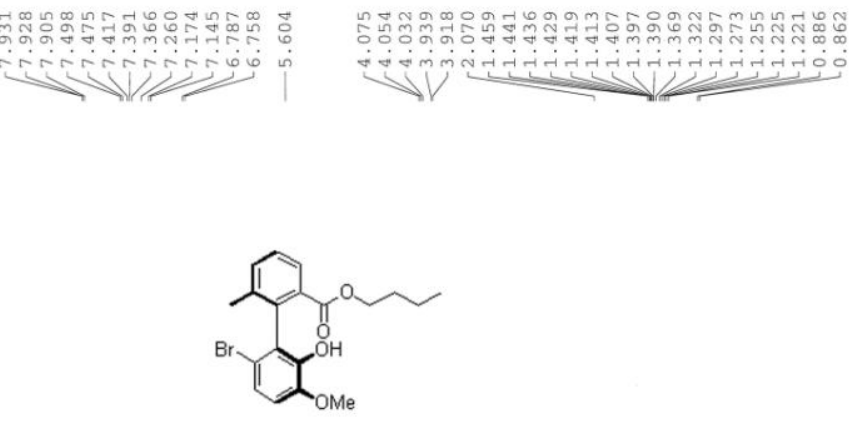

31
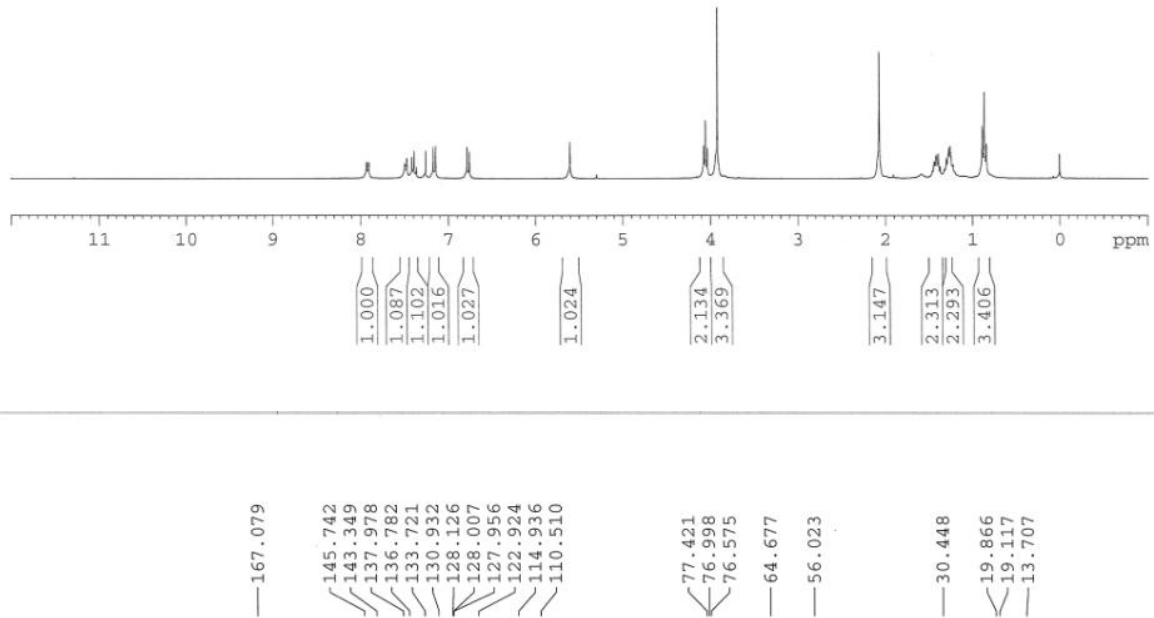

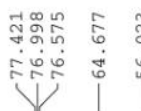

ఖ

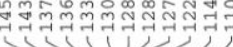

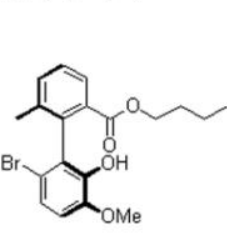

3I

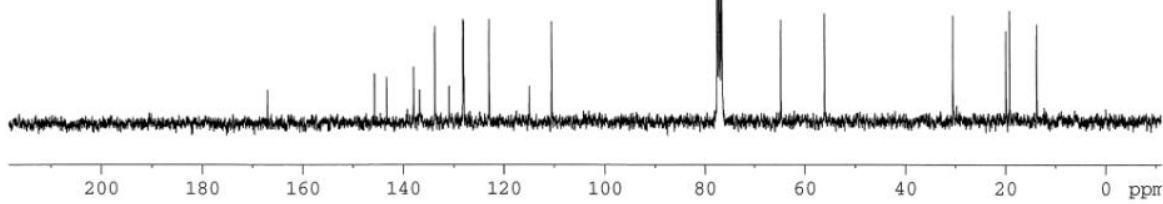



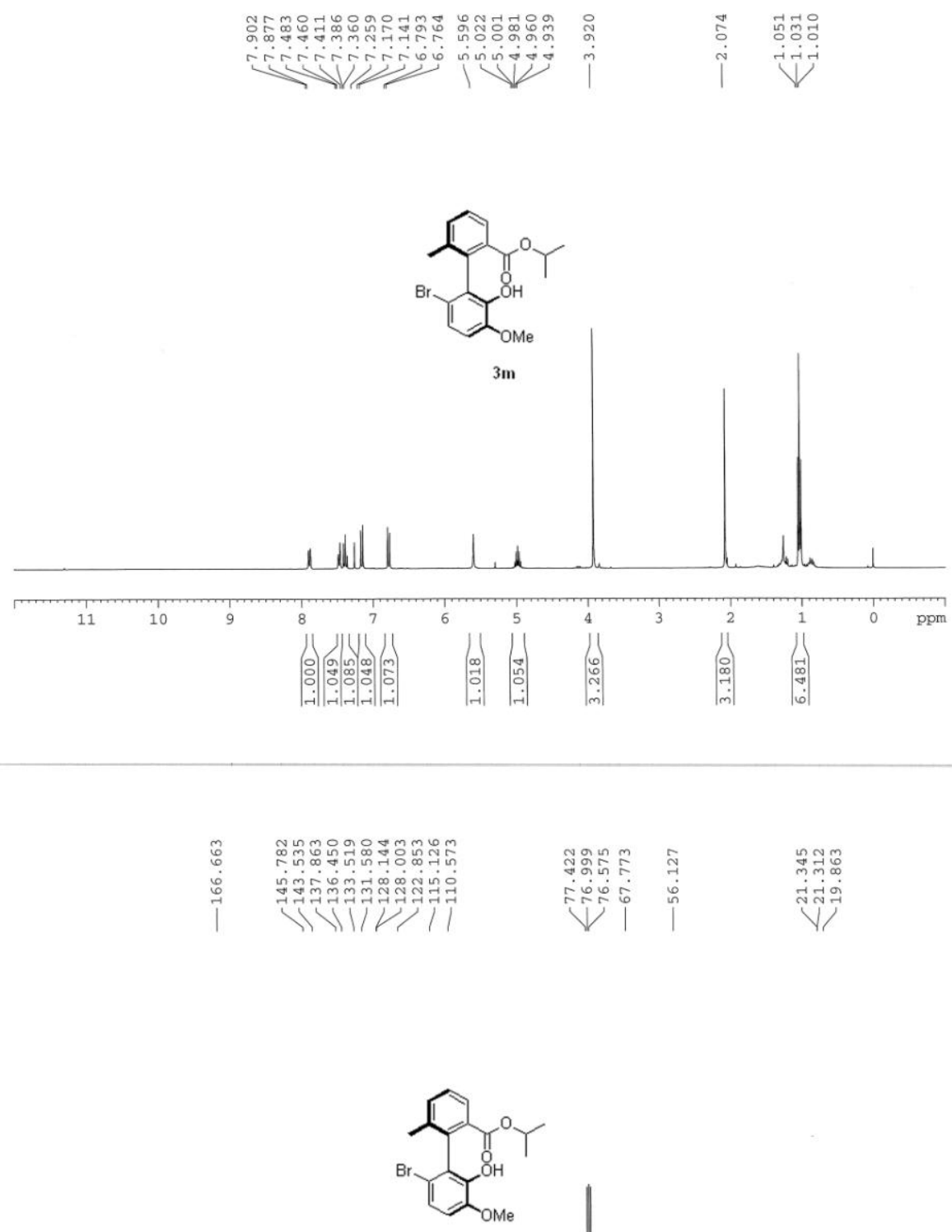

$3 \mathrm{~m}$ 


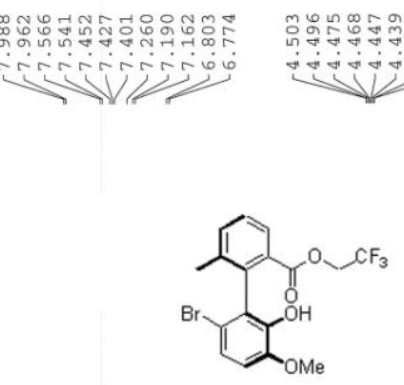

$3 n$
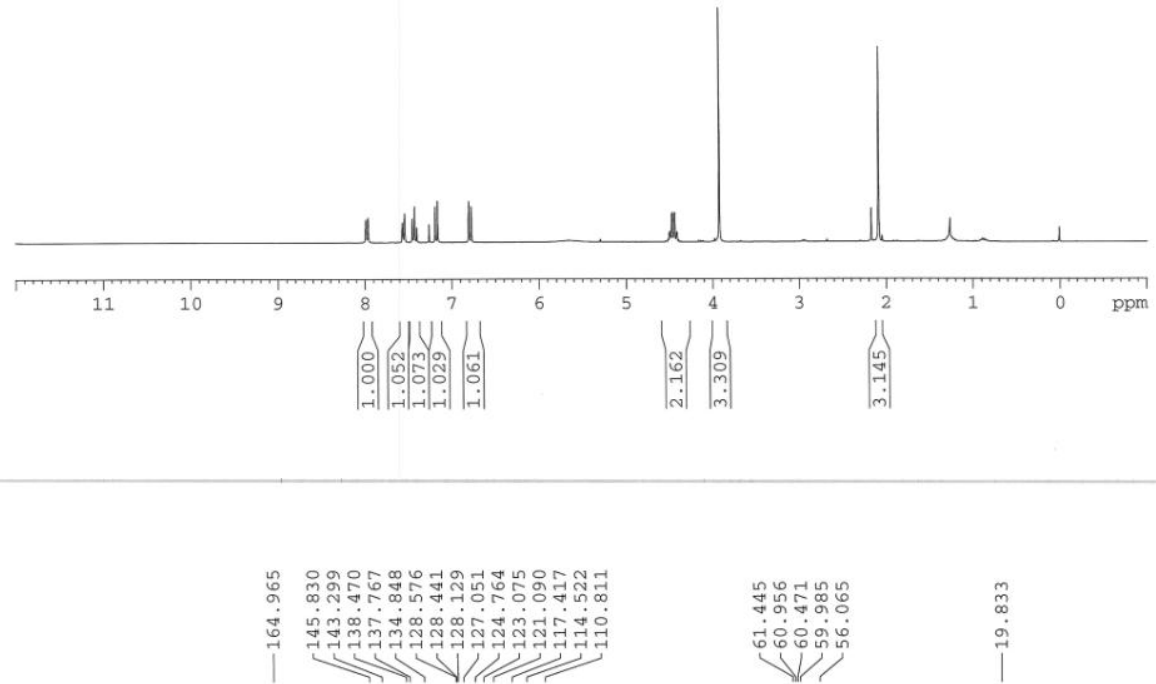

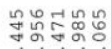

용ํํ

$\stackrel{\substack{n \\ \infty}}{\stackrel{\rho}{\mid}}$

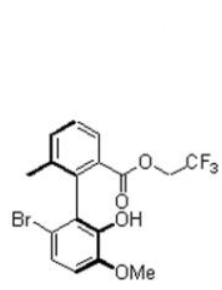

$3 n$

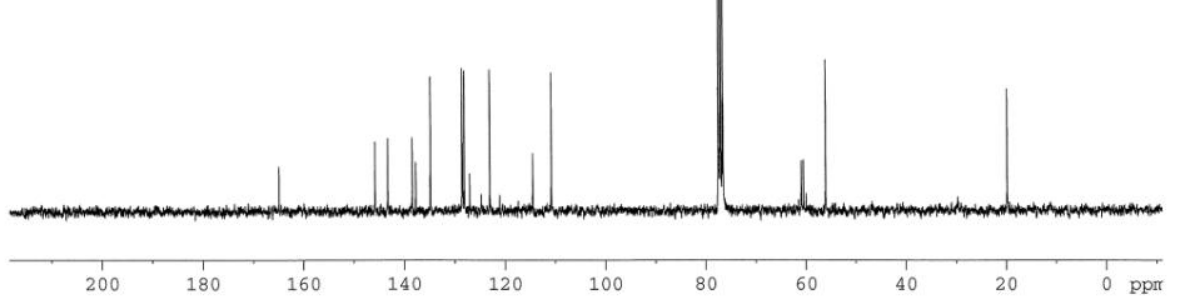



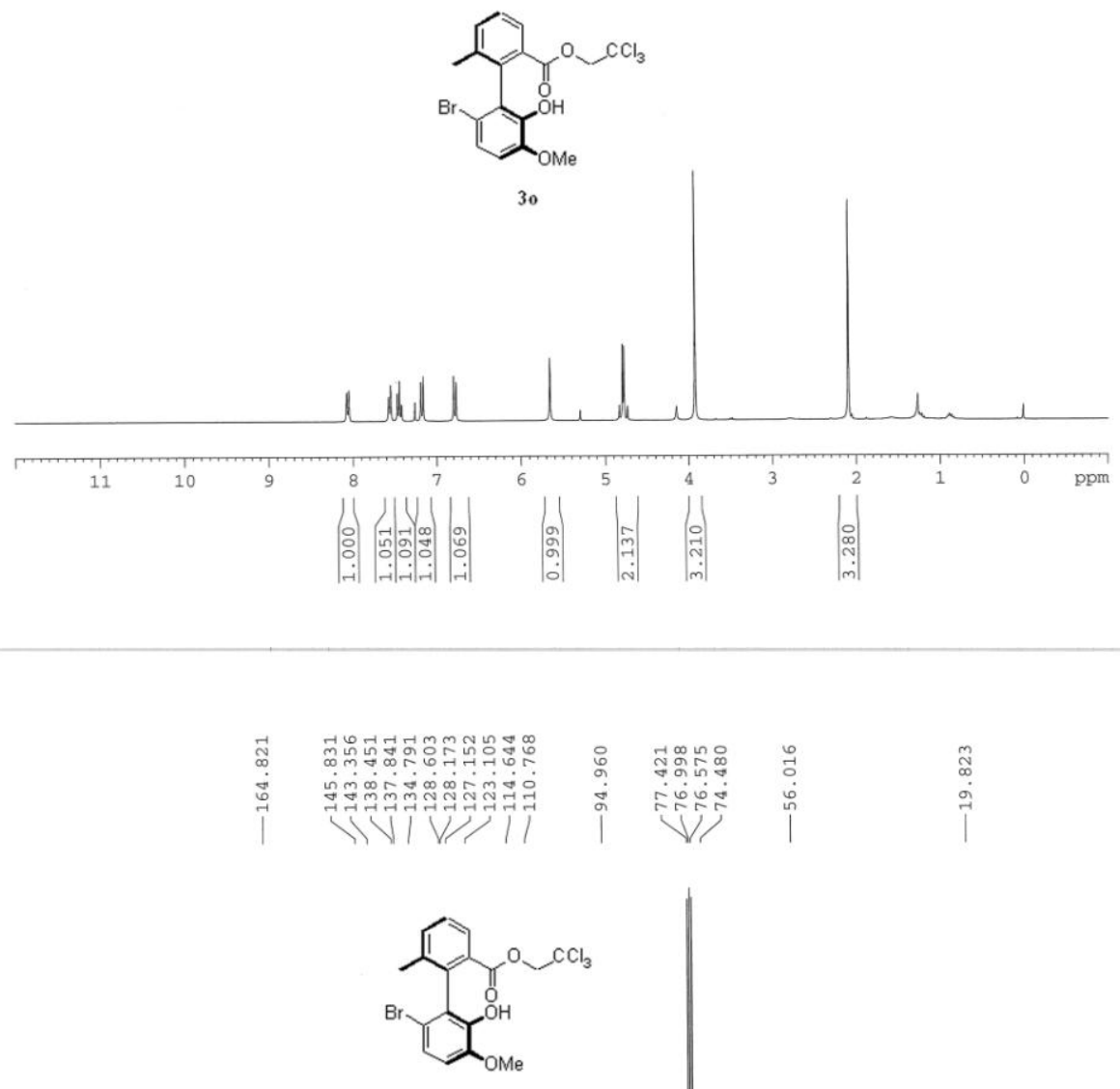

30

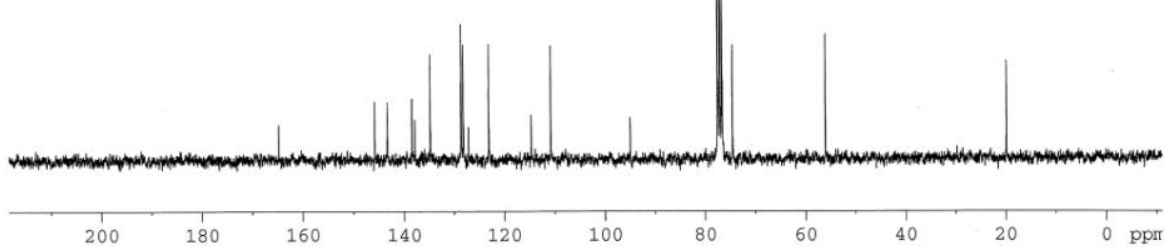




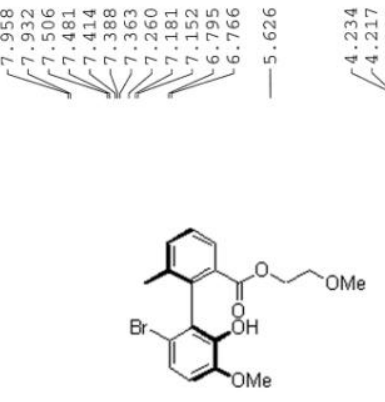

$3 p$
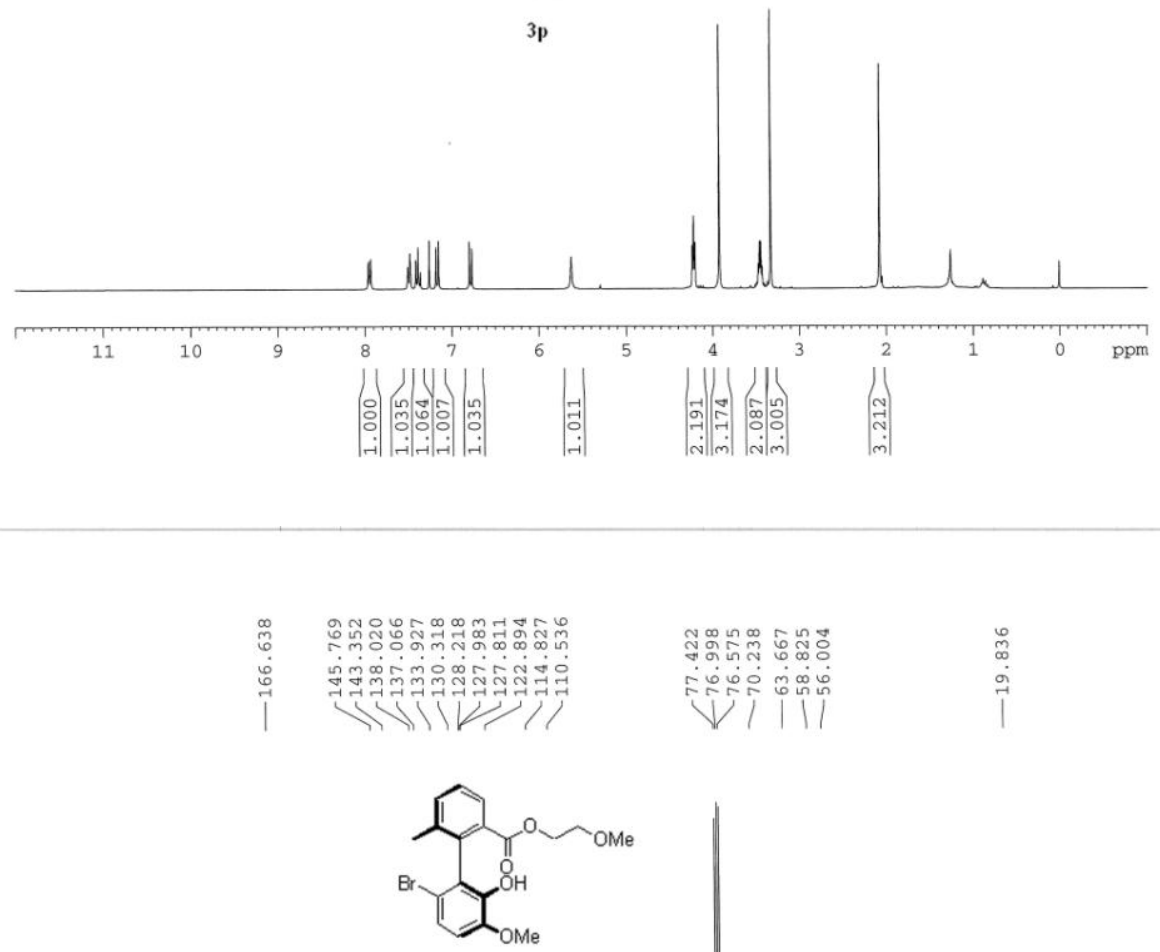

3p

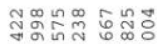

두유 꿍

ํํㅇ ।

|

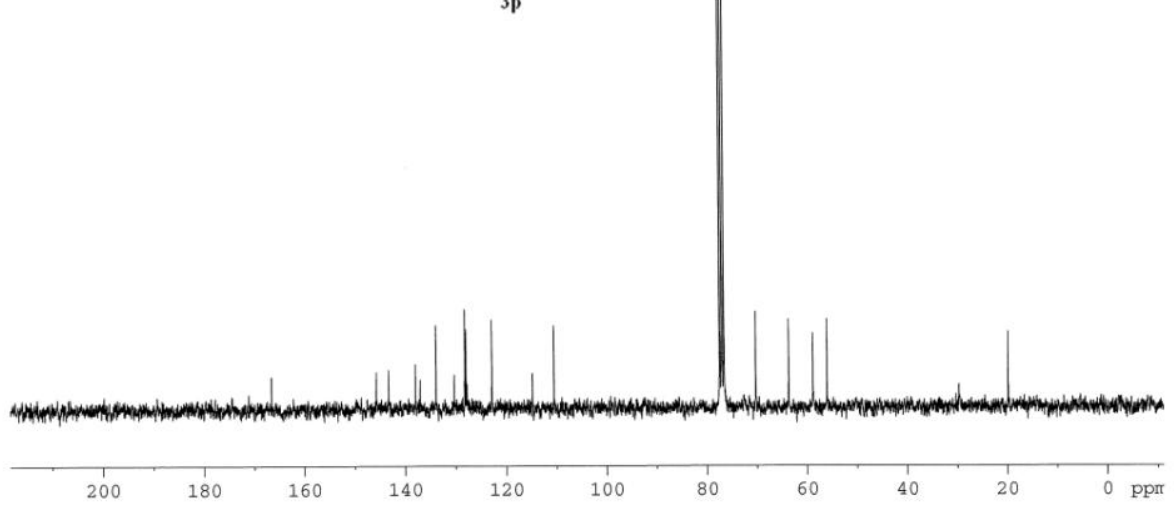



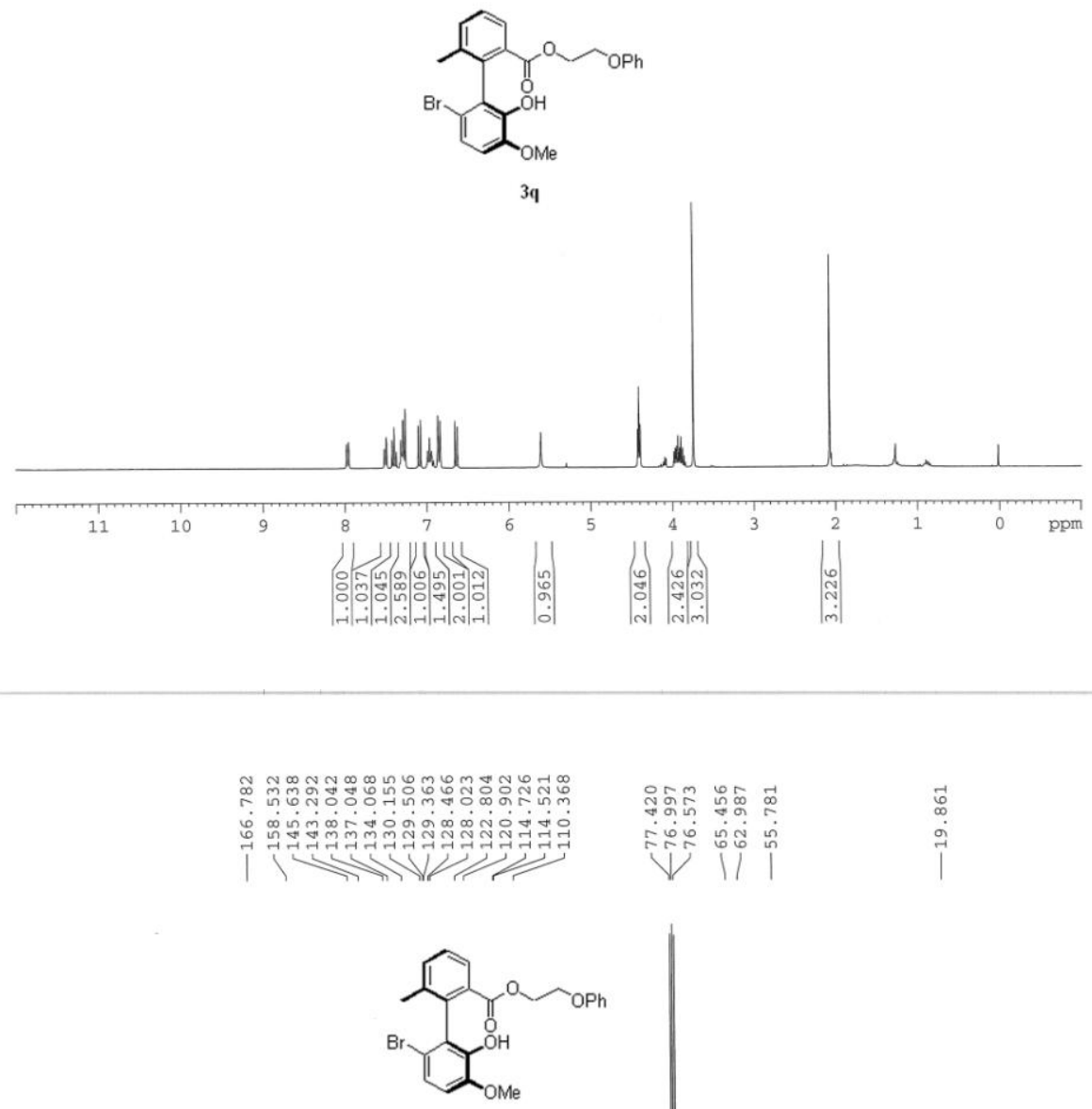

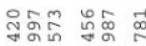

रํำ

$\overrightarrow{6}$

V 1111

$3 q$

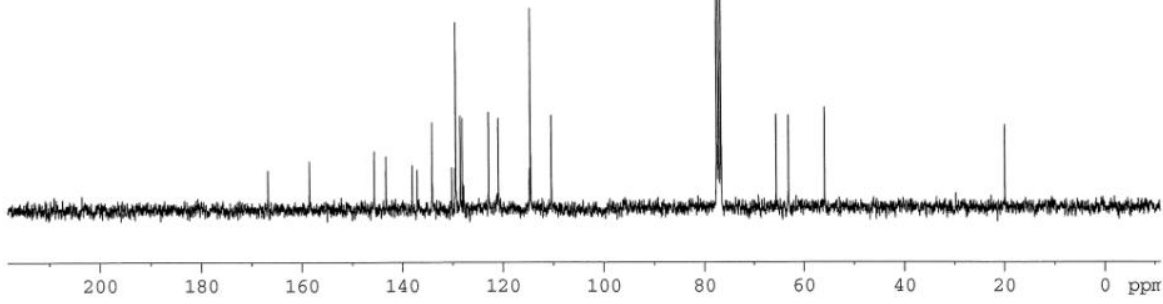



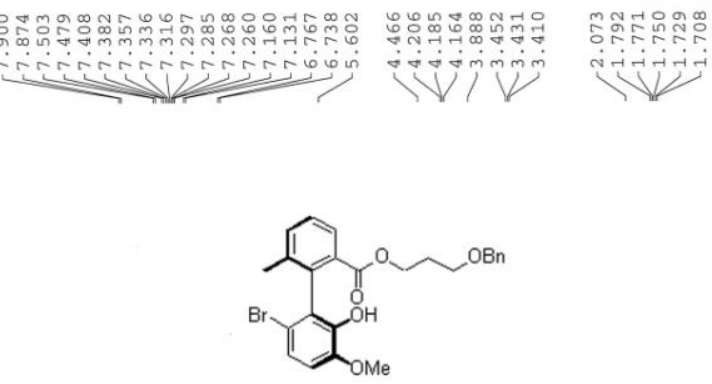

$3 \mathrm{r}$
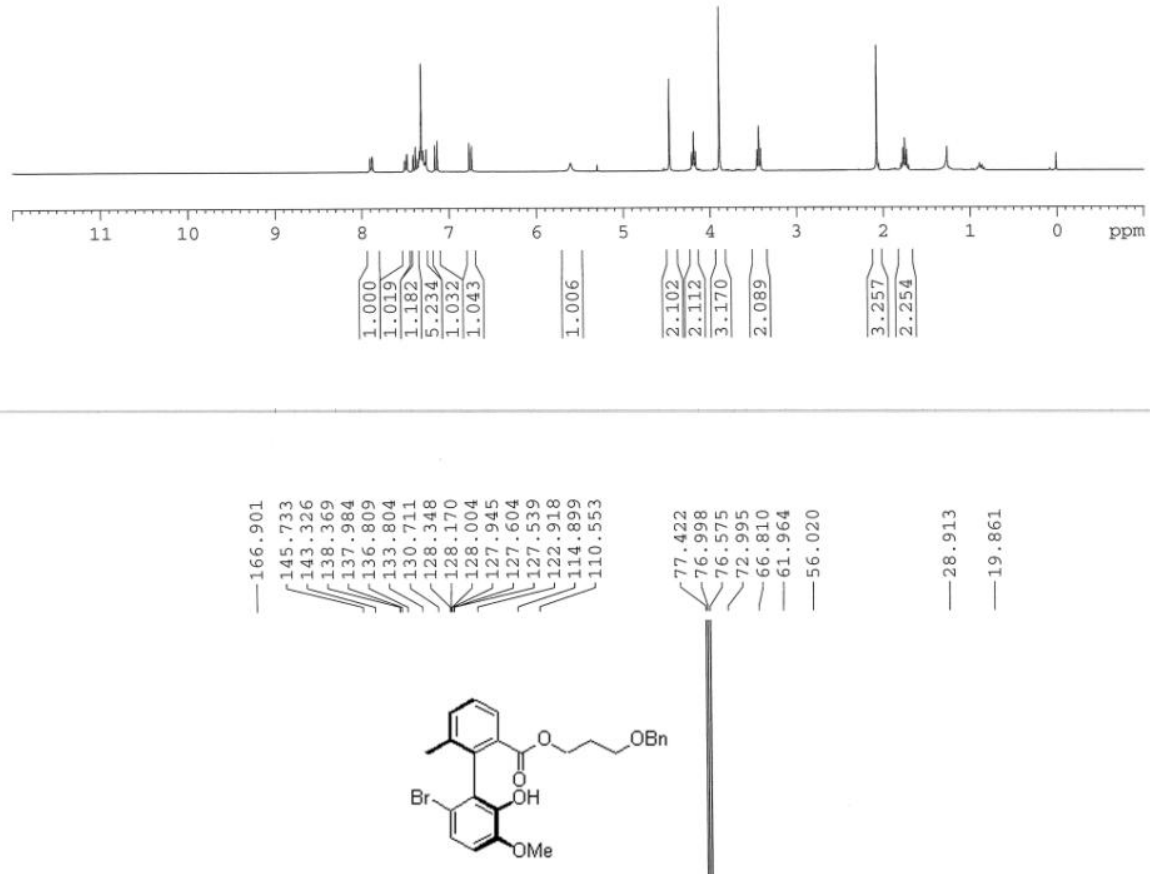

3r

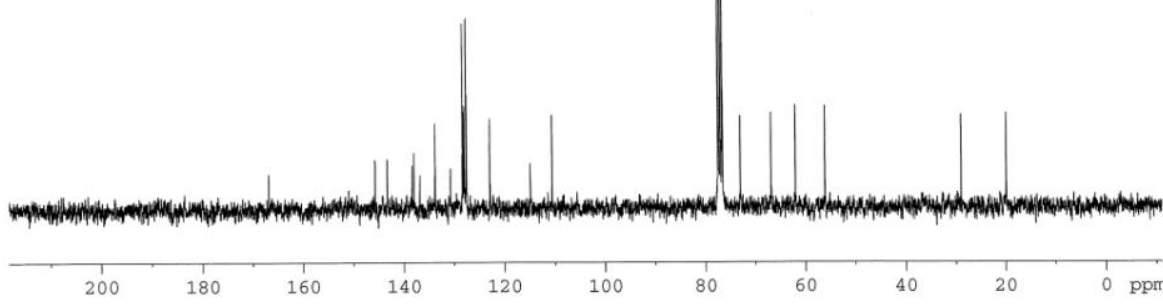



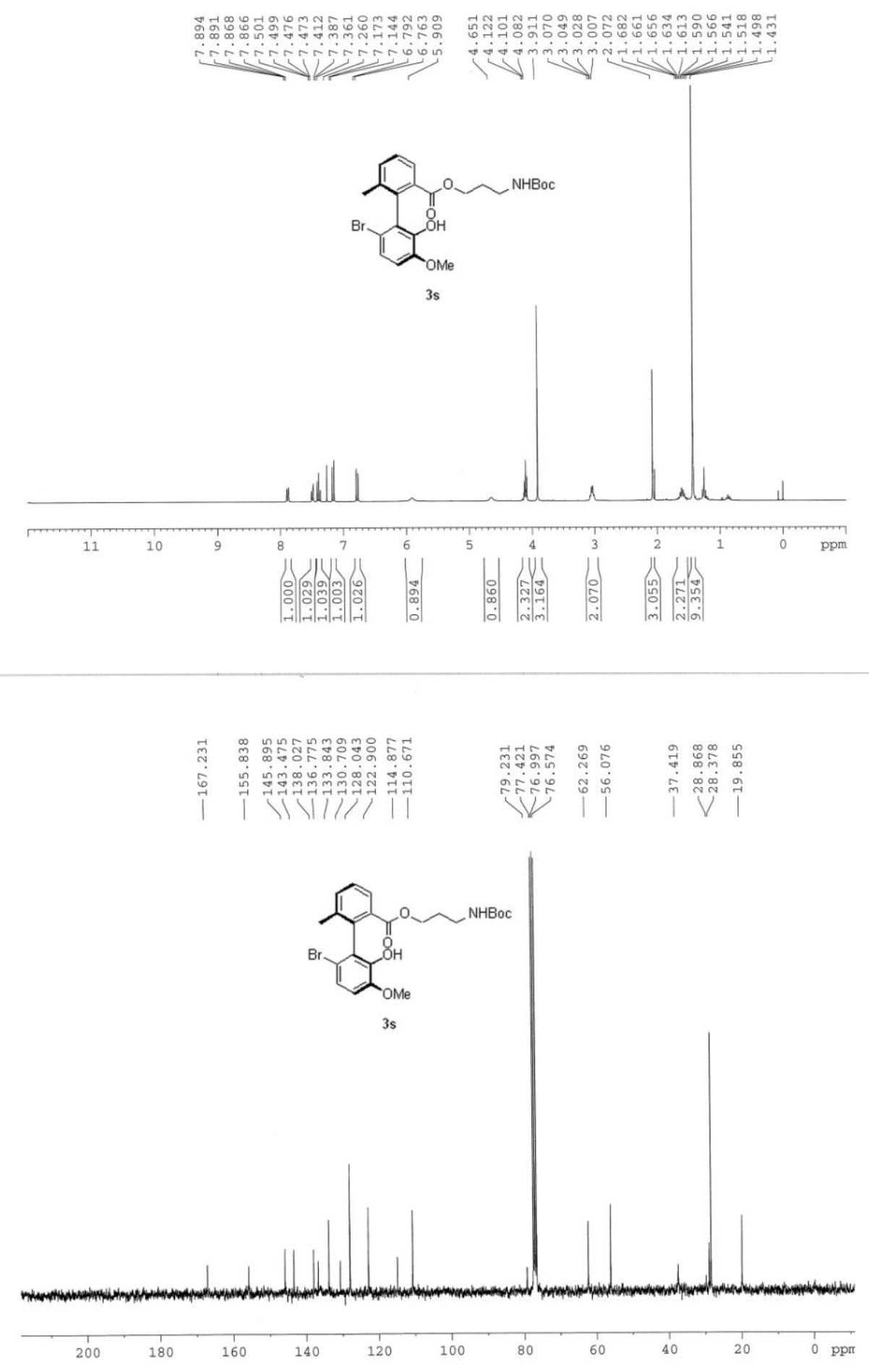


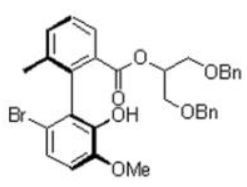

$3 t$
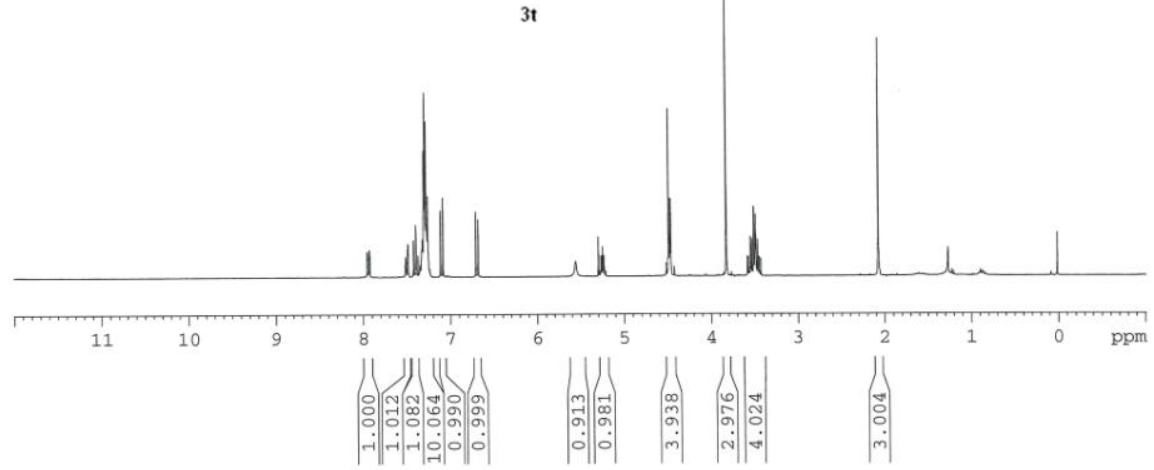

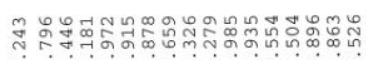

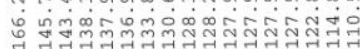

(

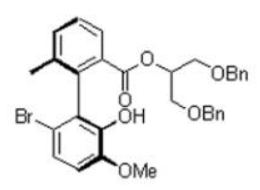

$3 \mathrm{t}$

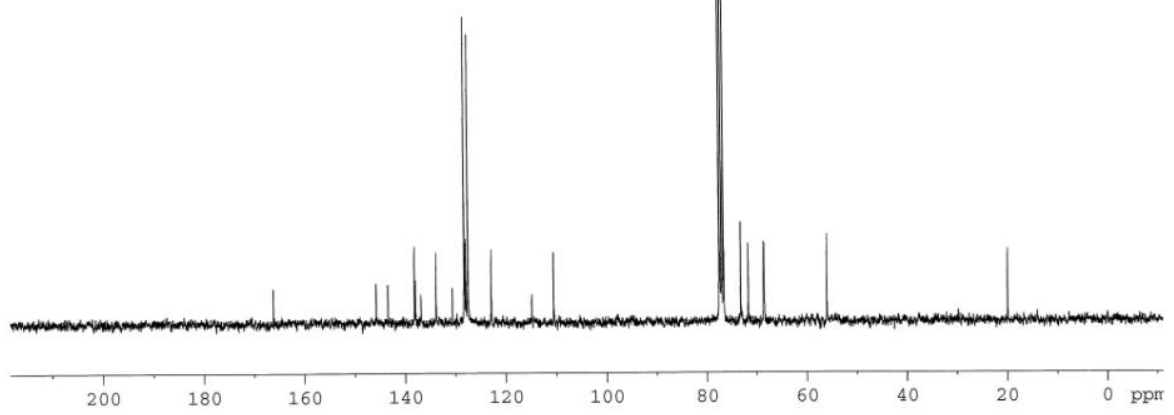



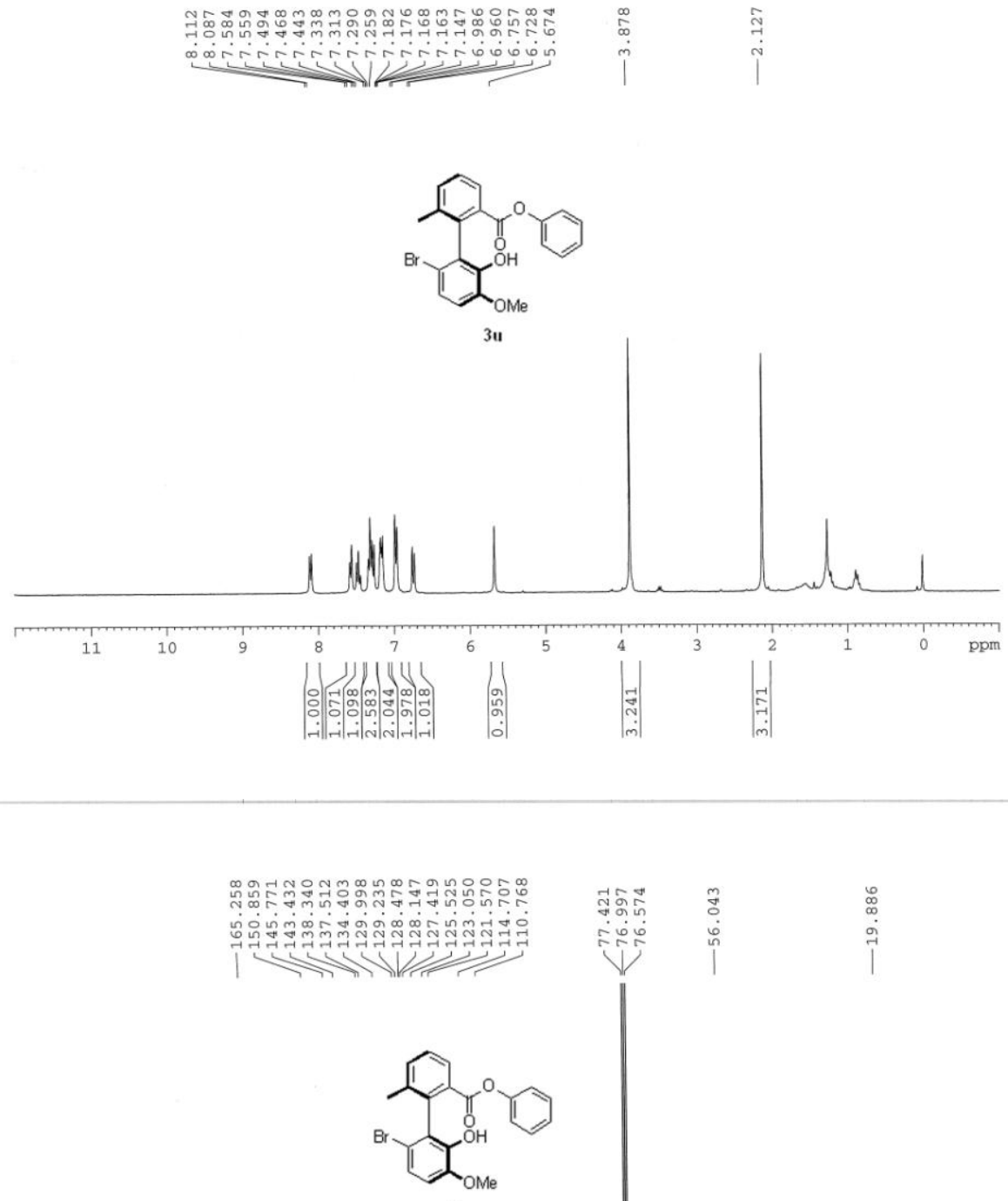

3 u

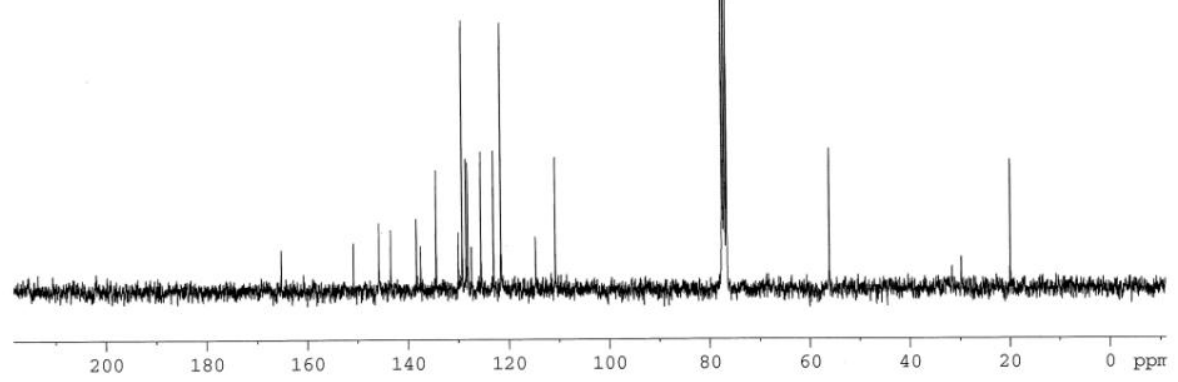



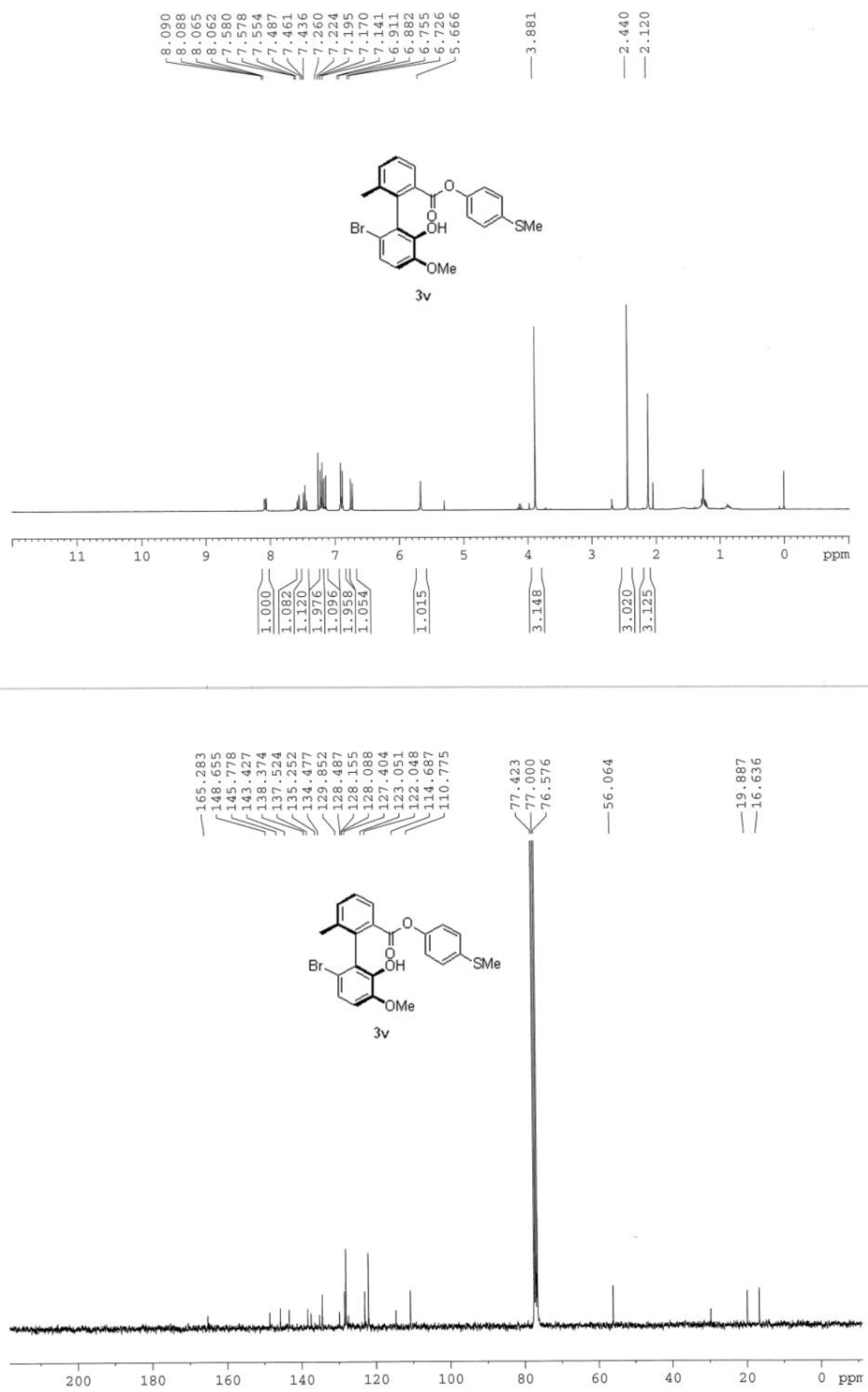


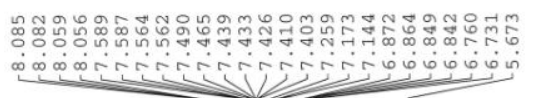

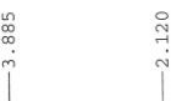

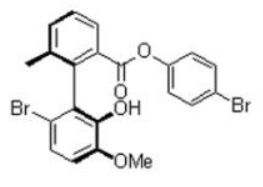

$3 w$
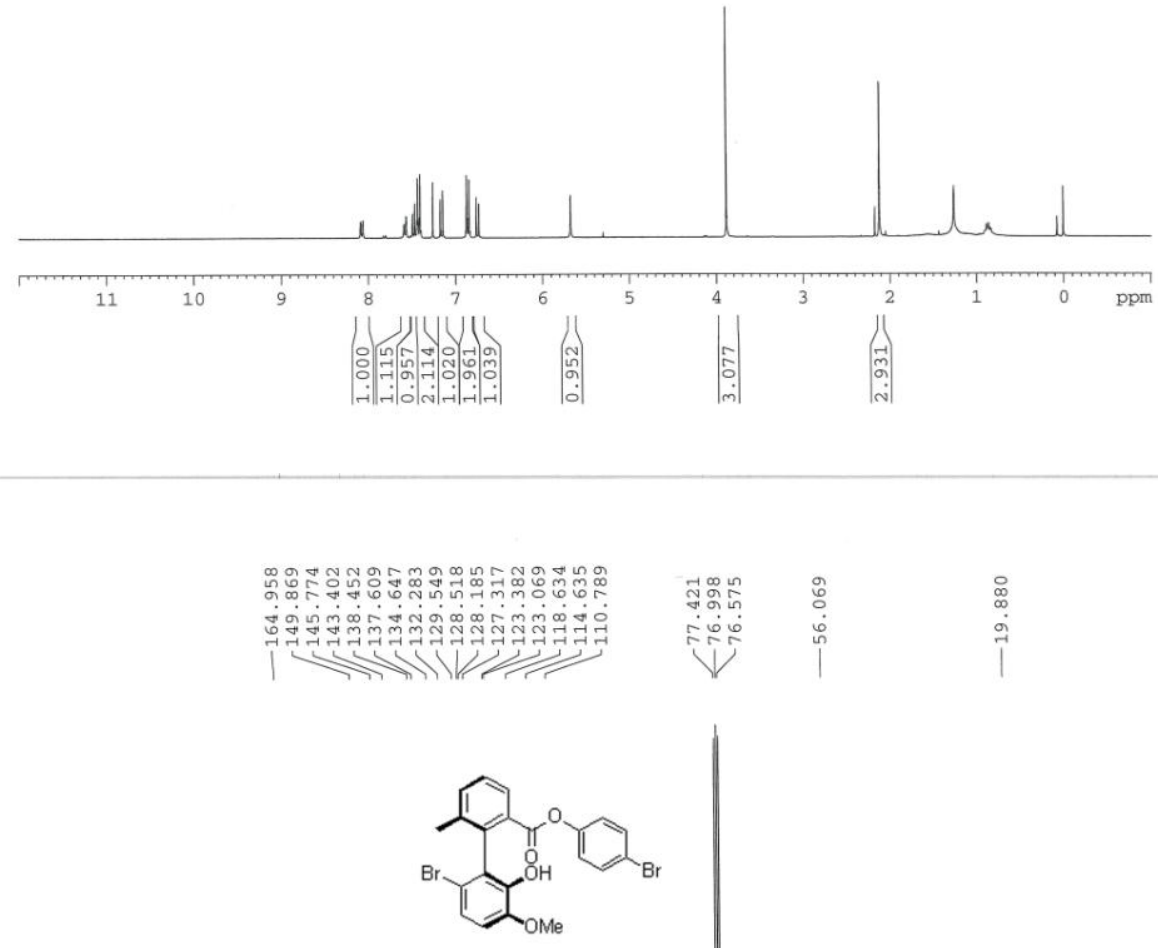

$3 w$

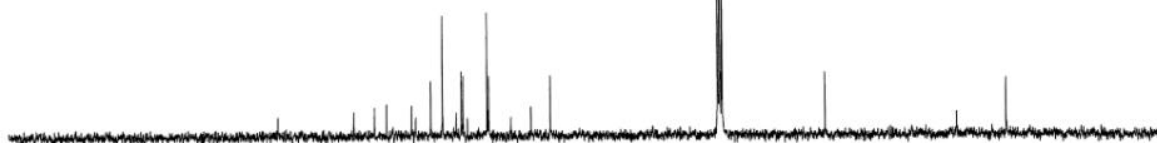

200

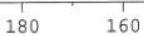

60

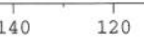

100

60

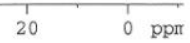



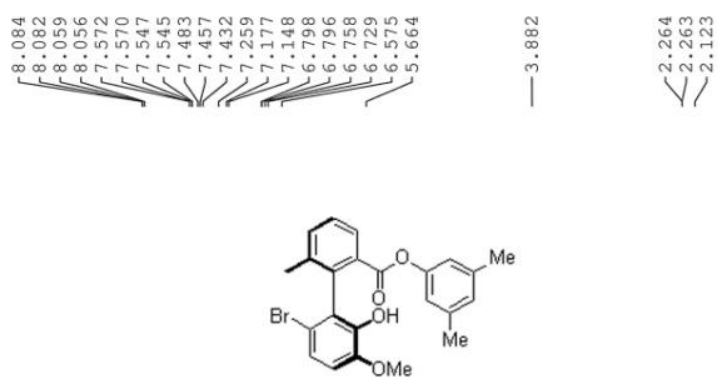

$3 x$
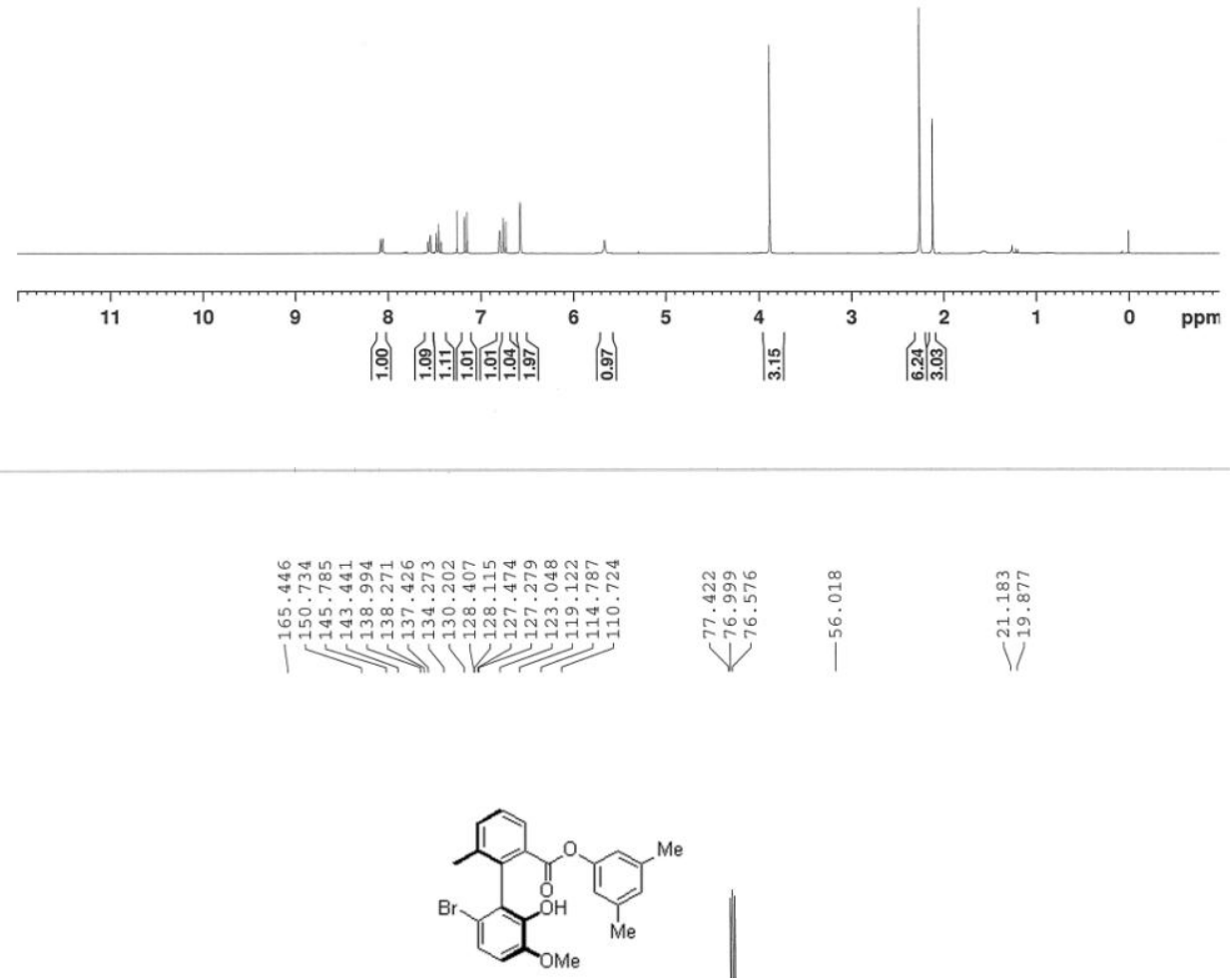

$3 x$

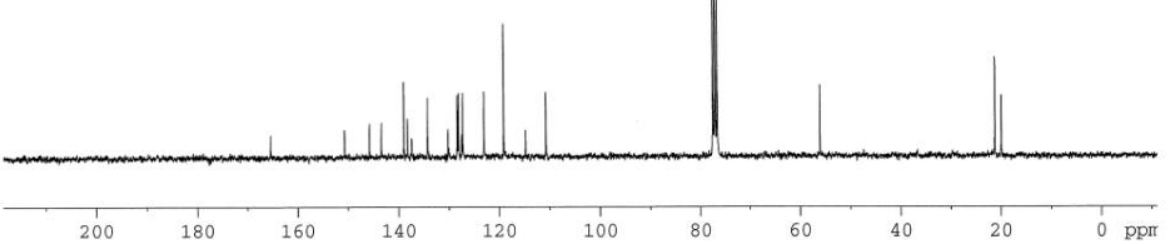




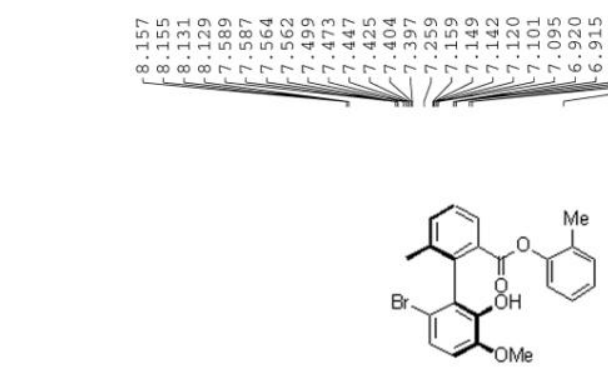

3y
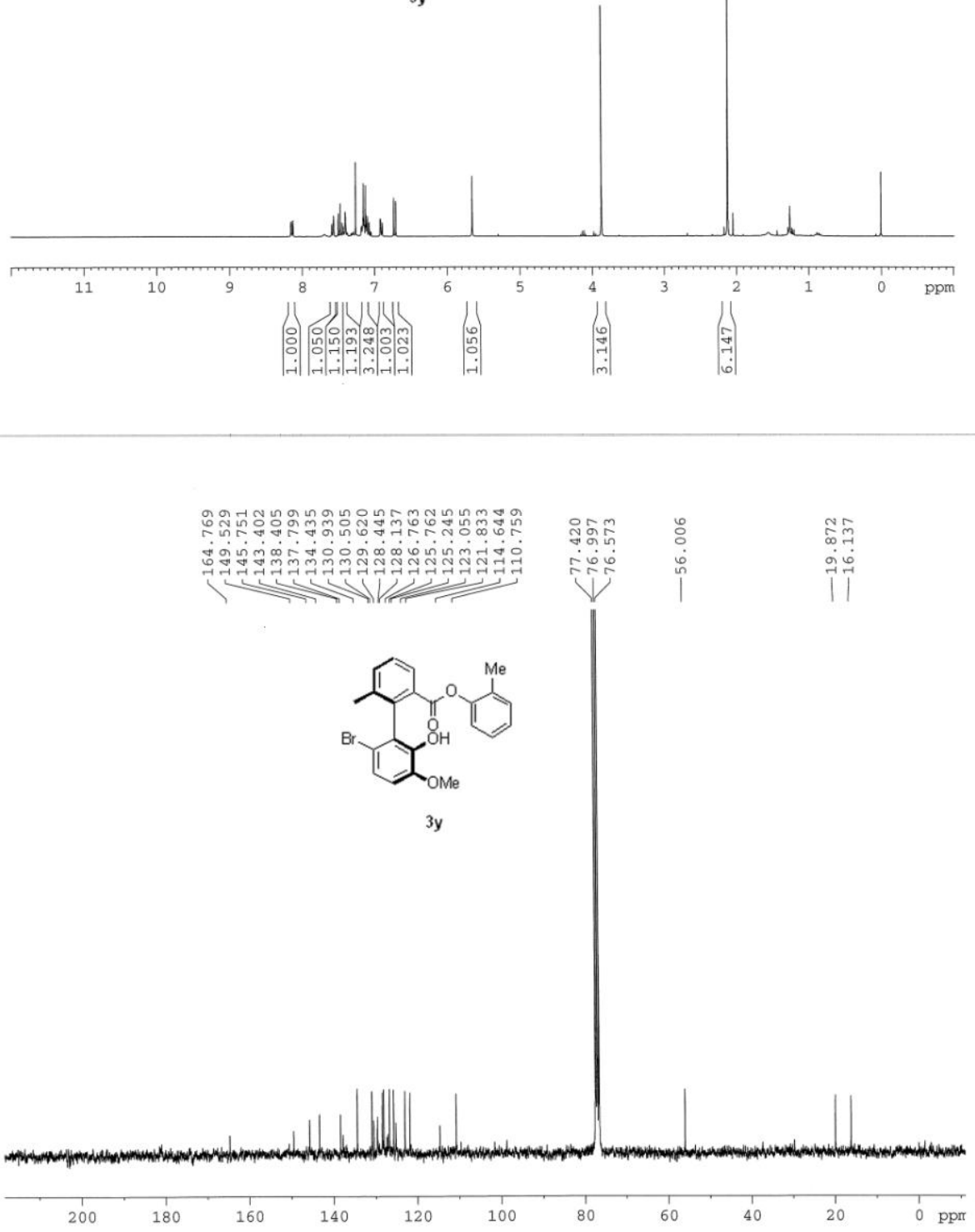

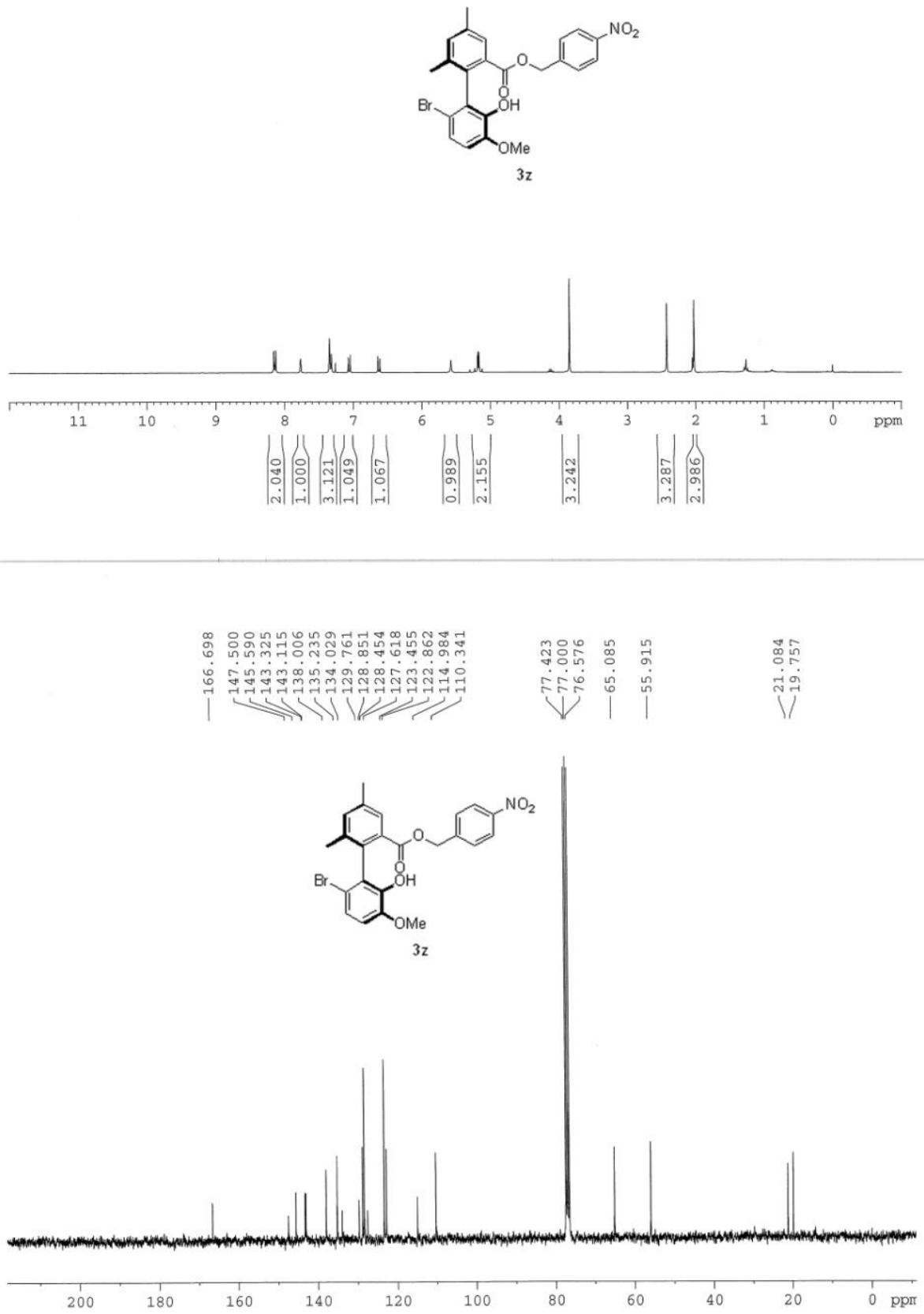


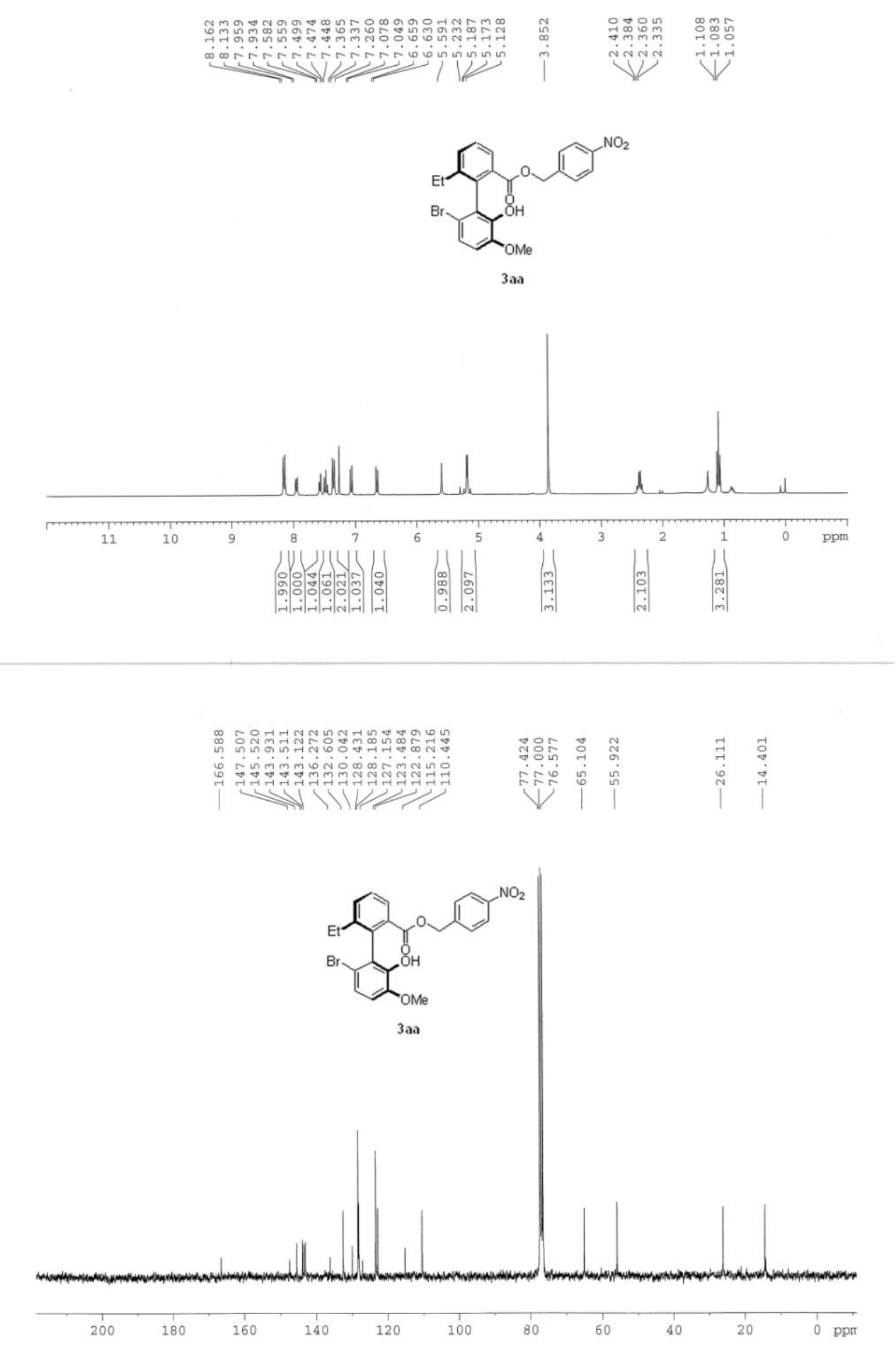




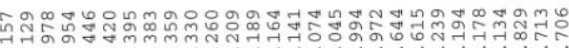

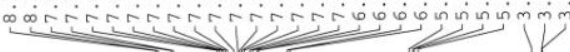

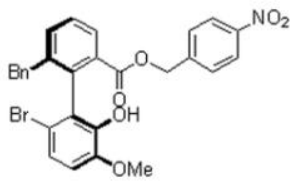

3ab
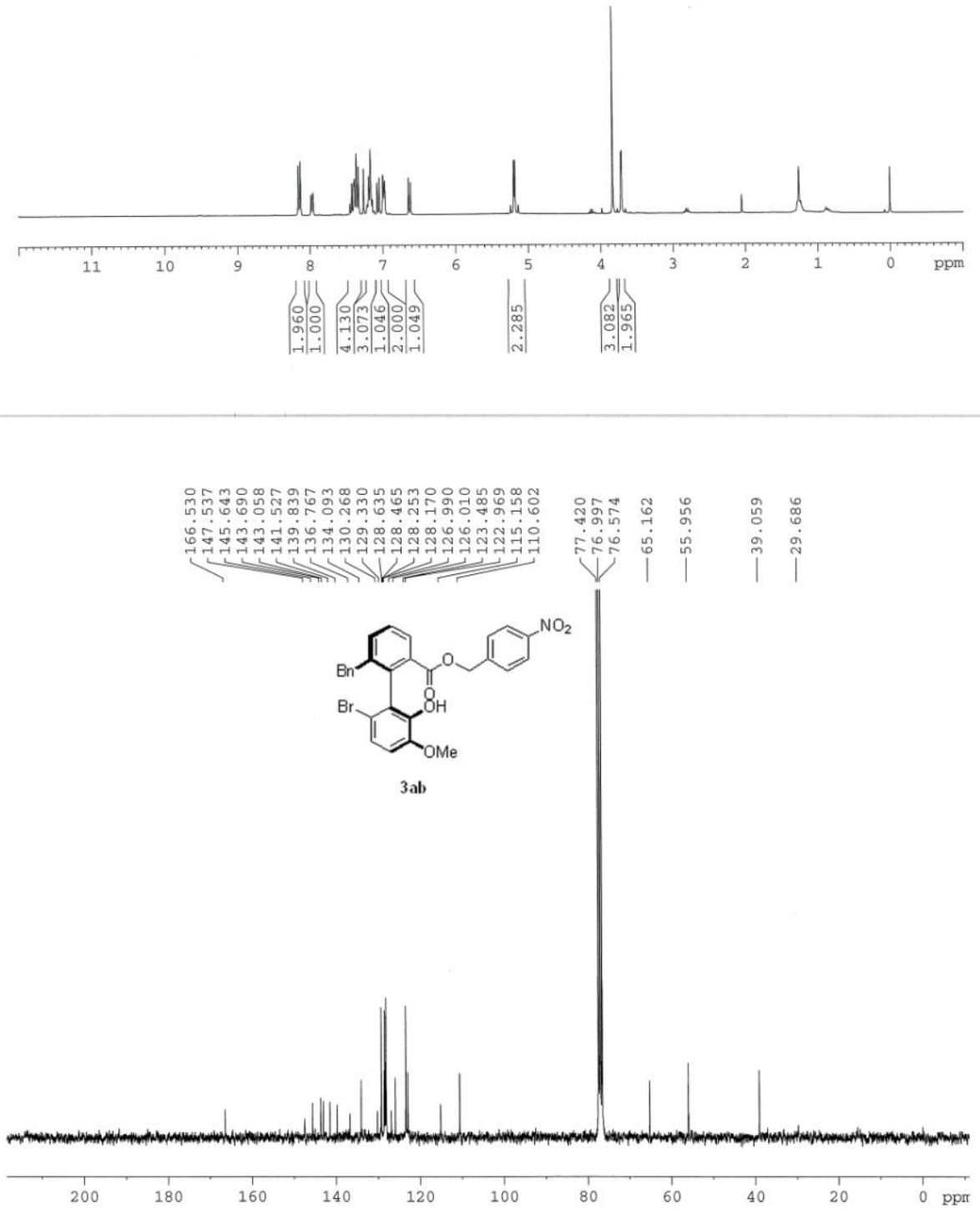

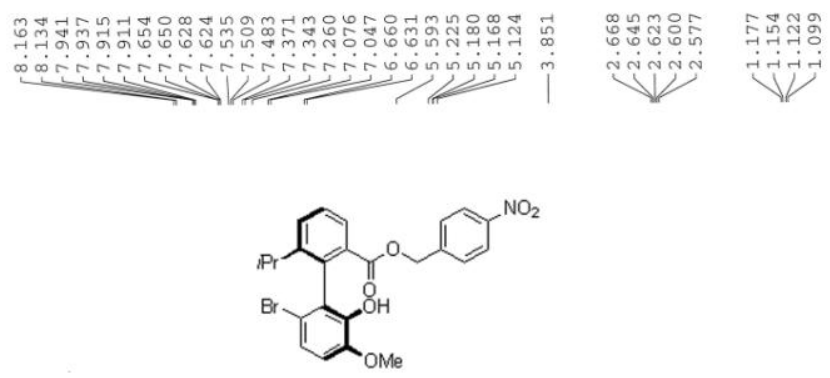

$3 \mathrm{ac}$
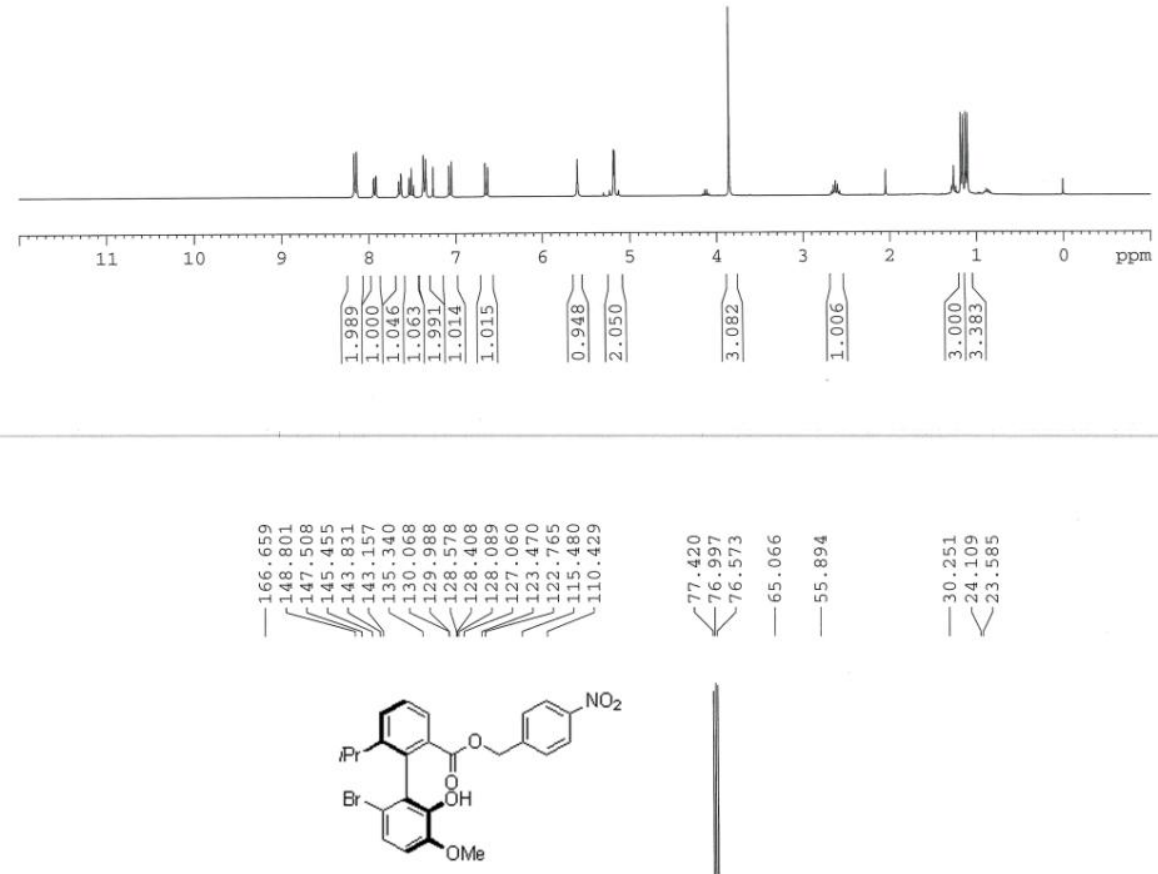

$3 \mathrm{ac}$

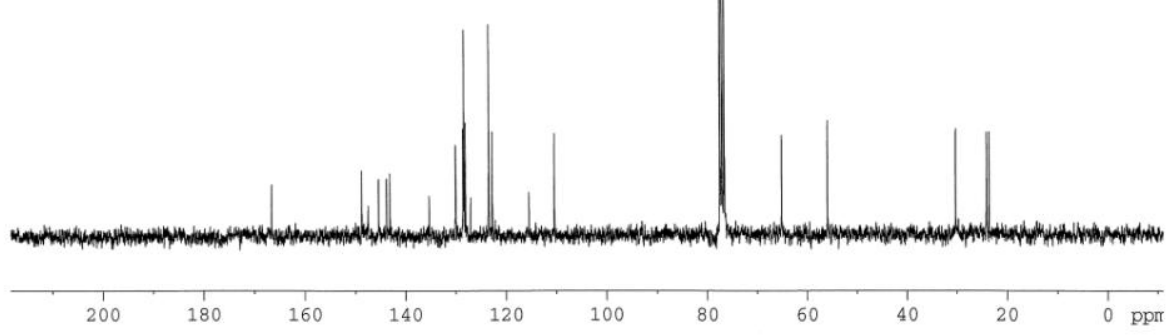




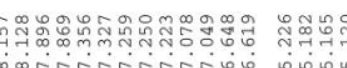

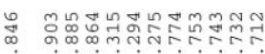

onisinitisio ning
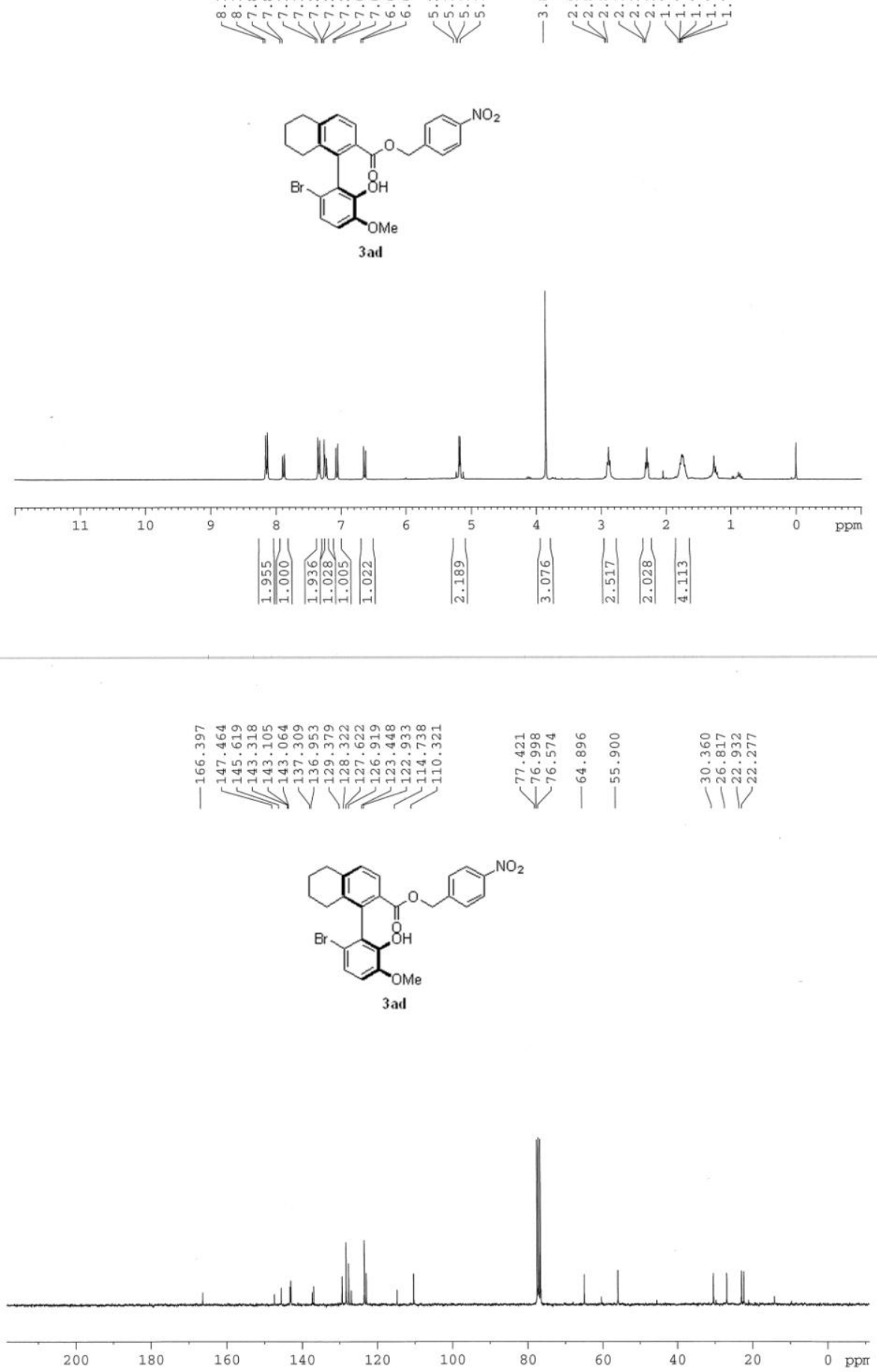


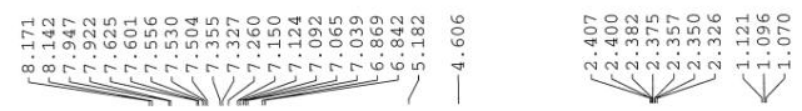

(nae
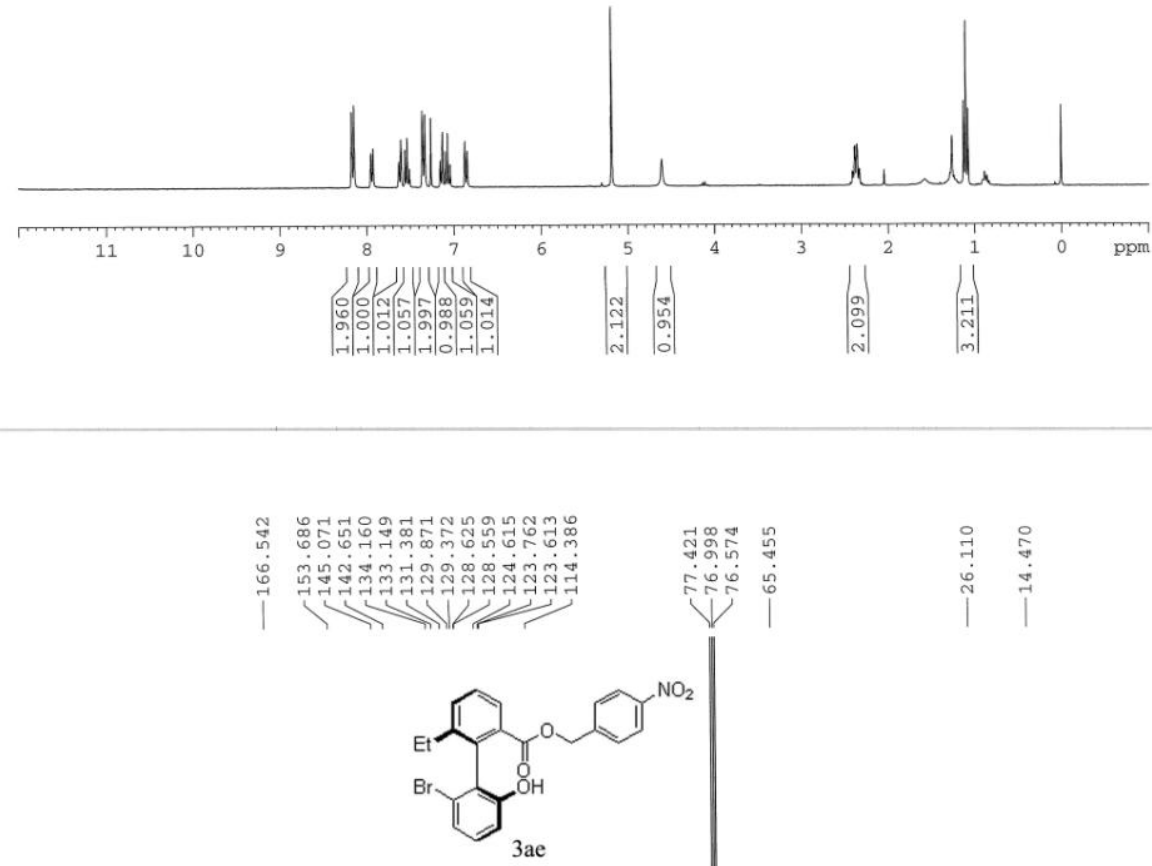

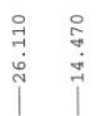

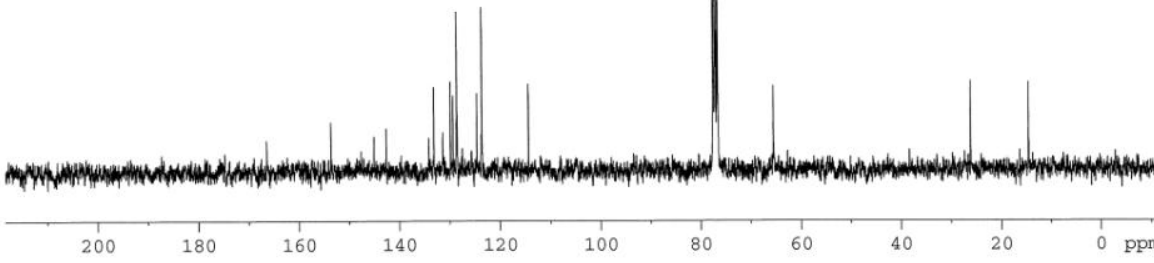




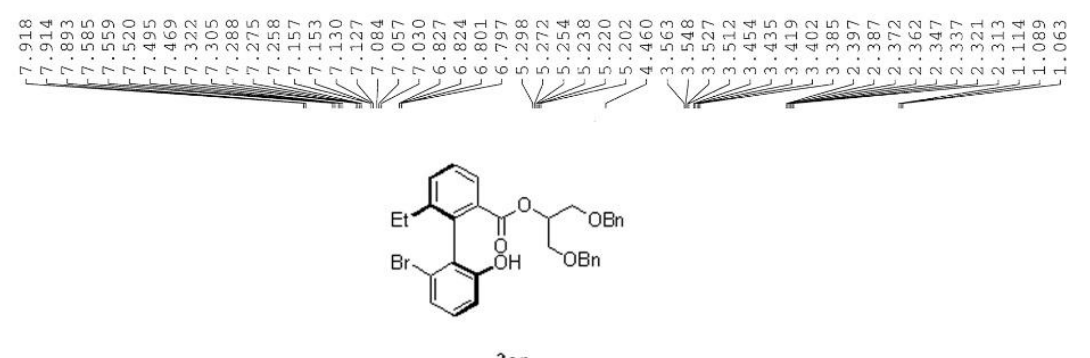

3 ag
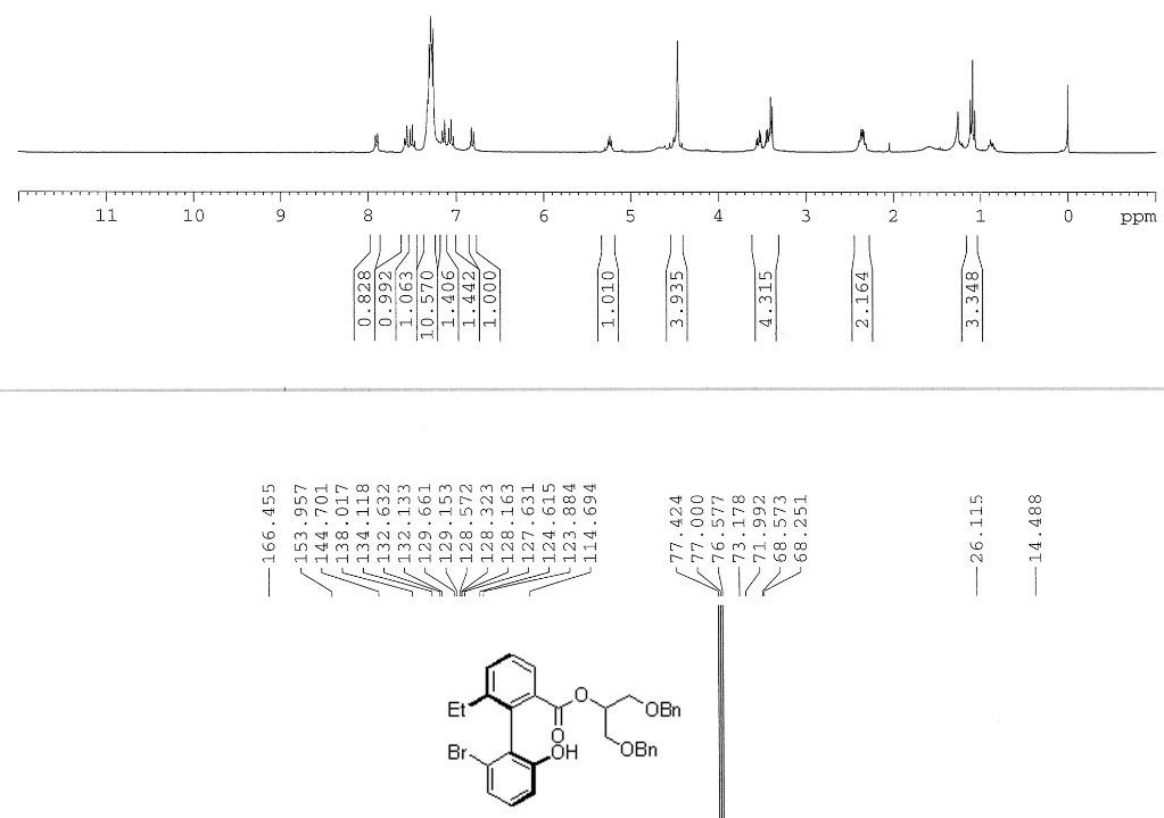

$3 a g$

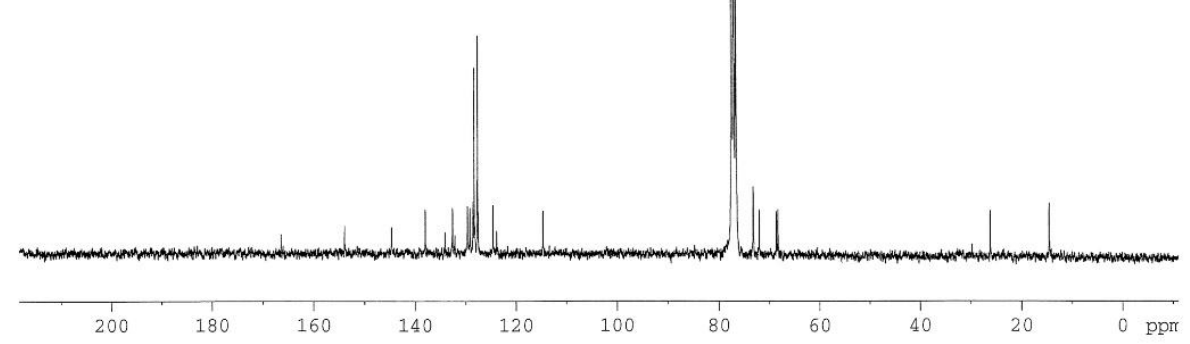




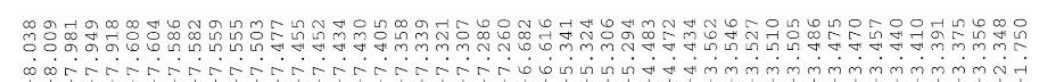

めarrar

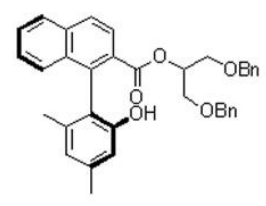

$3 a h$

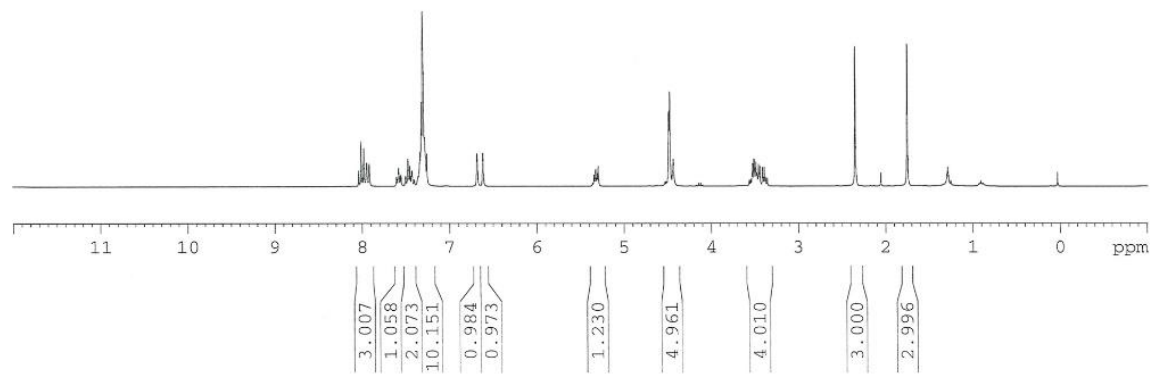

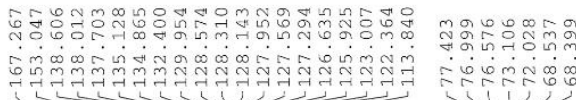

ֻ

1/

तั่

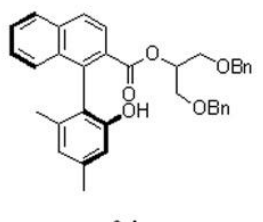

3 ah

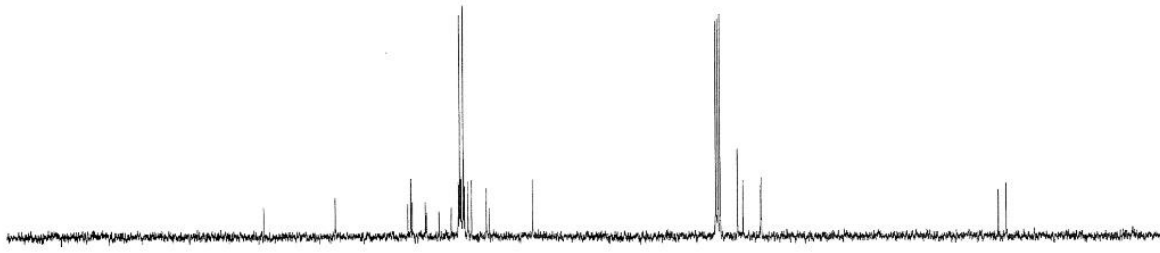

$200 \quad 180 \quad 160 \quad 140 \quad 120$

80

60

40

$20 \quad 0$ ppr 

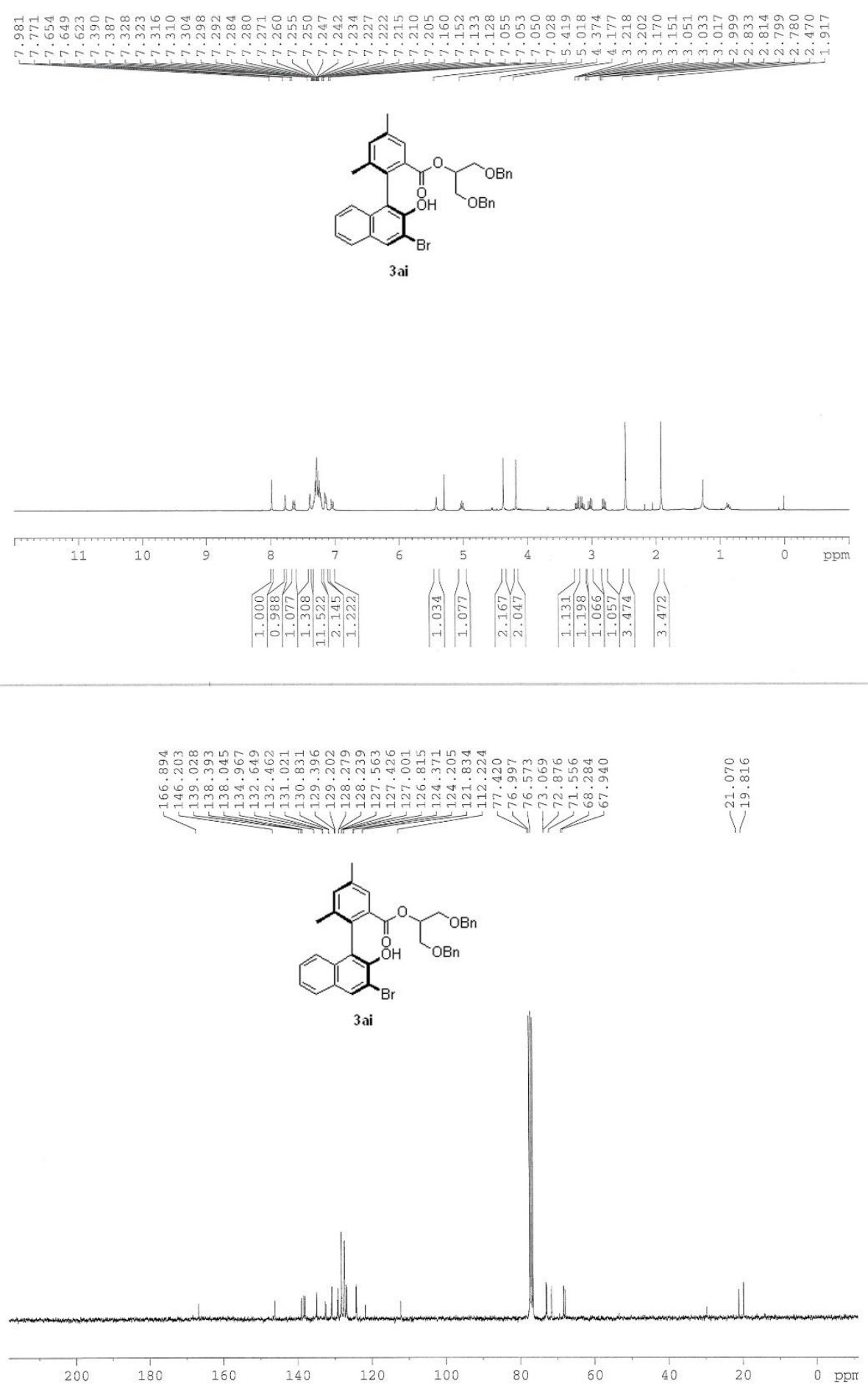

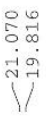




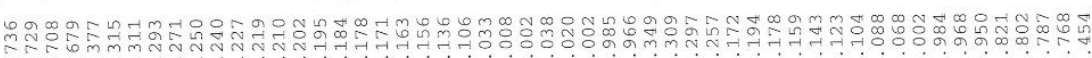

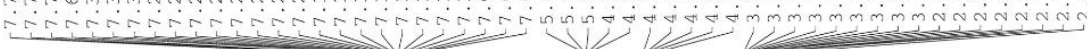

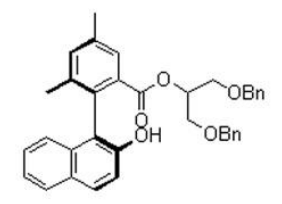

3aj

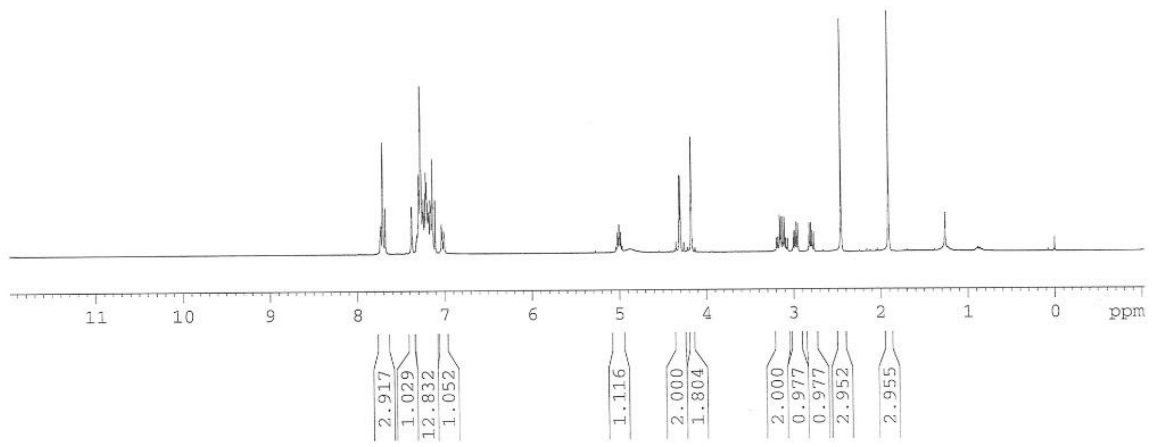

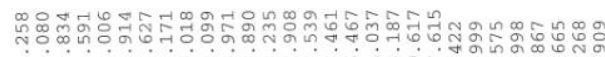

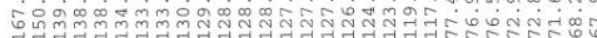

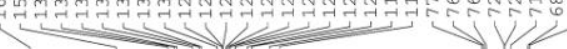

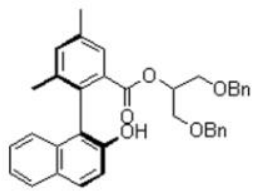

3 ạ

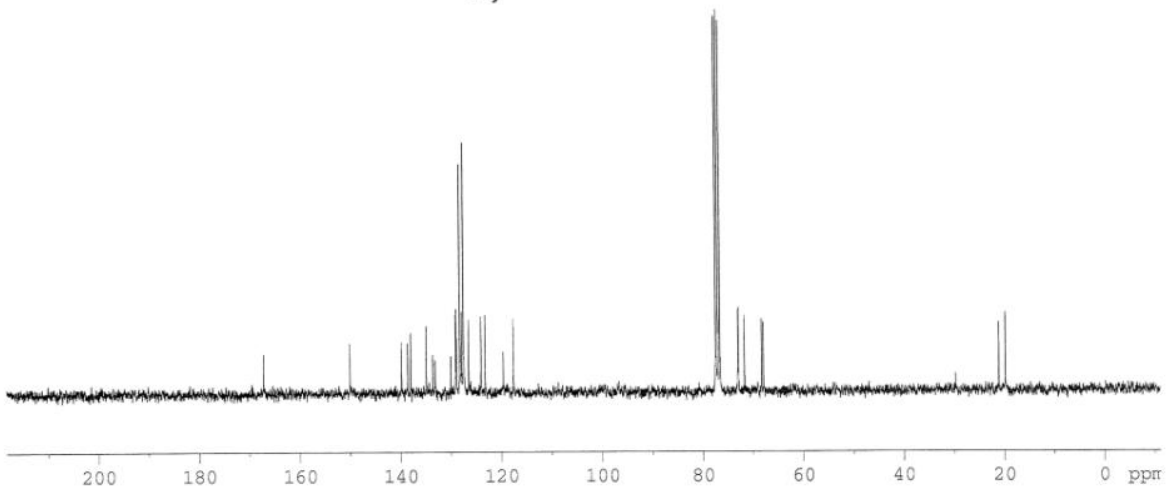




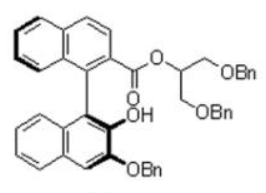

$3 \mathrm{ak}$

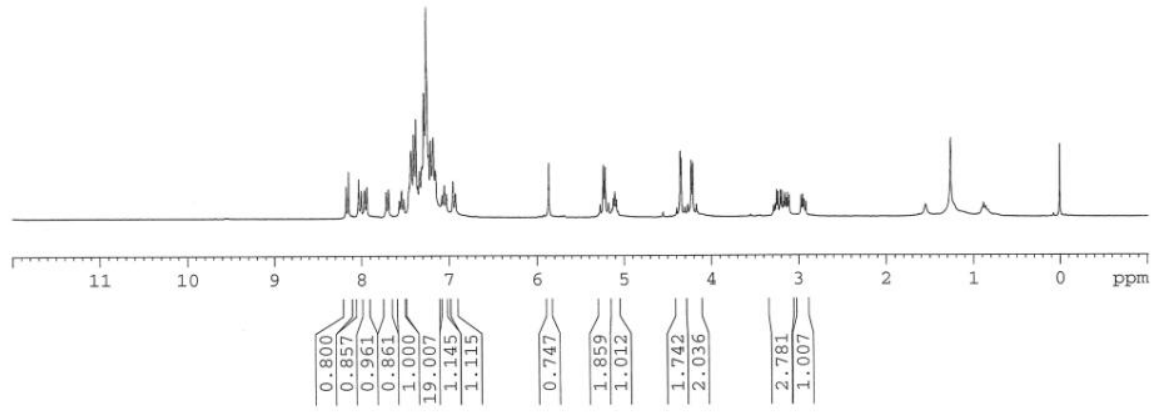

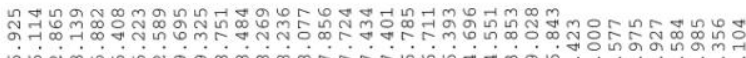

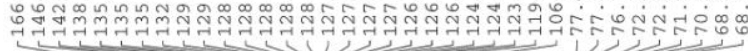

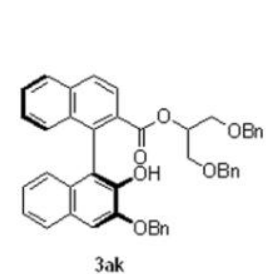

3ak

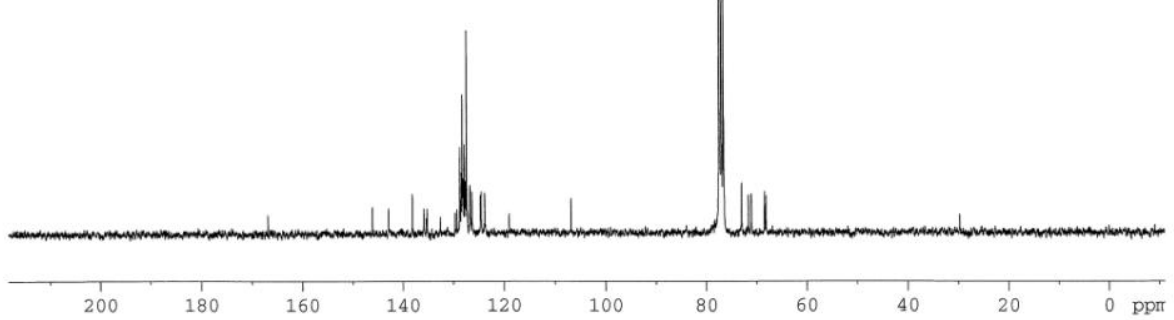



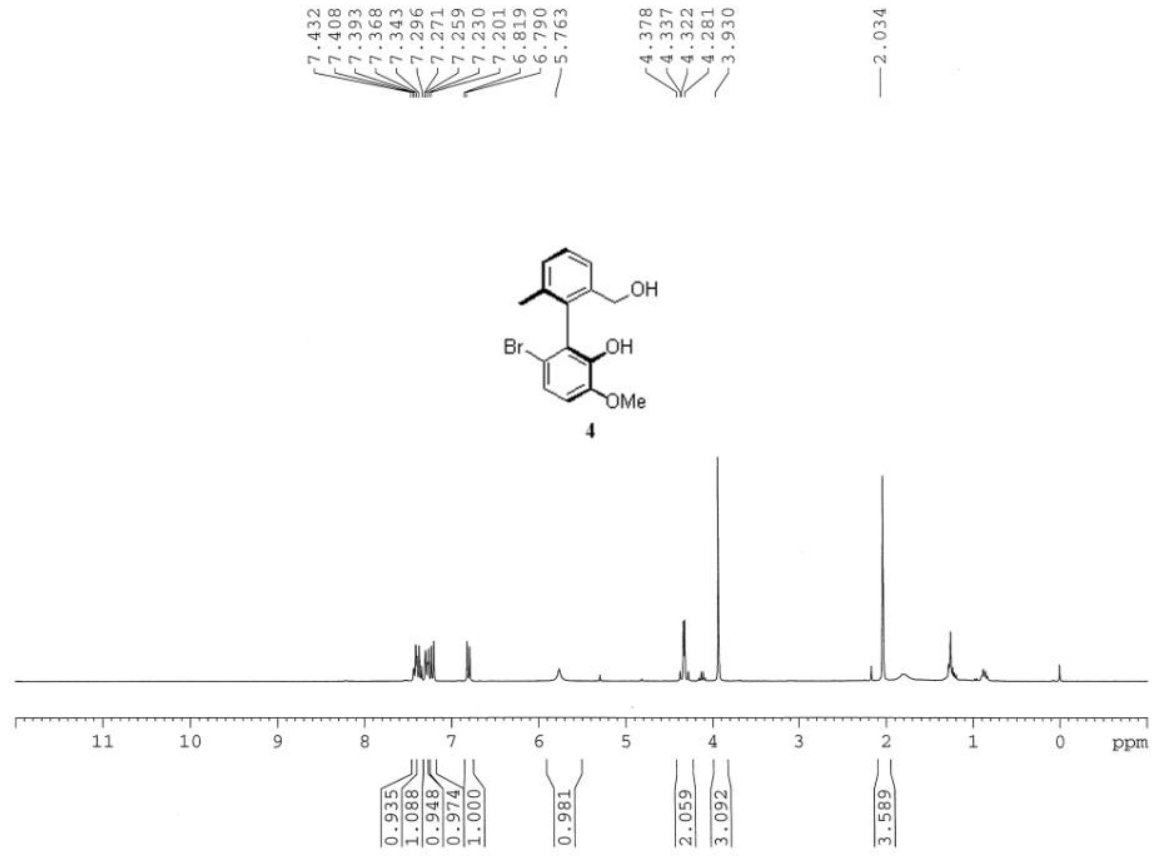

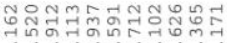

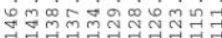

गा।

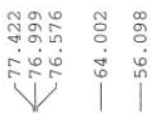

|

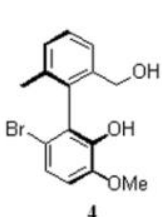

4

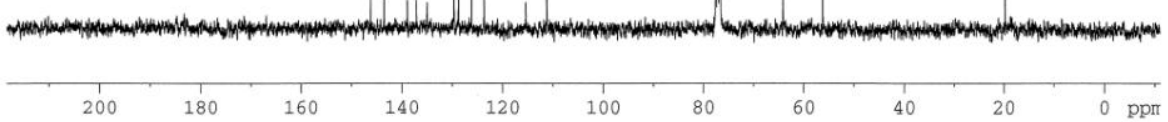




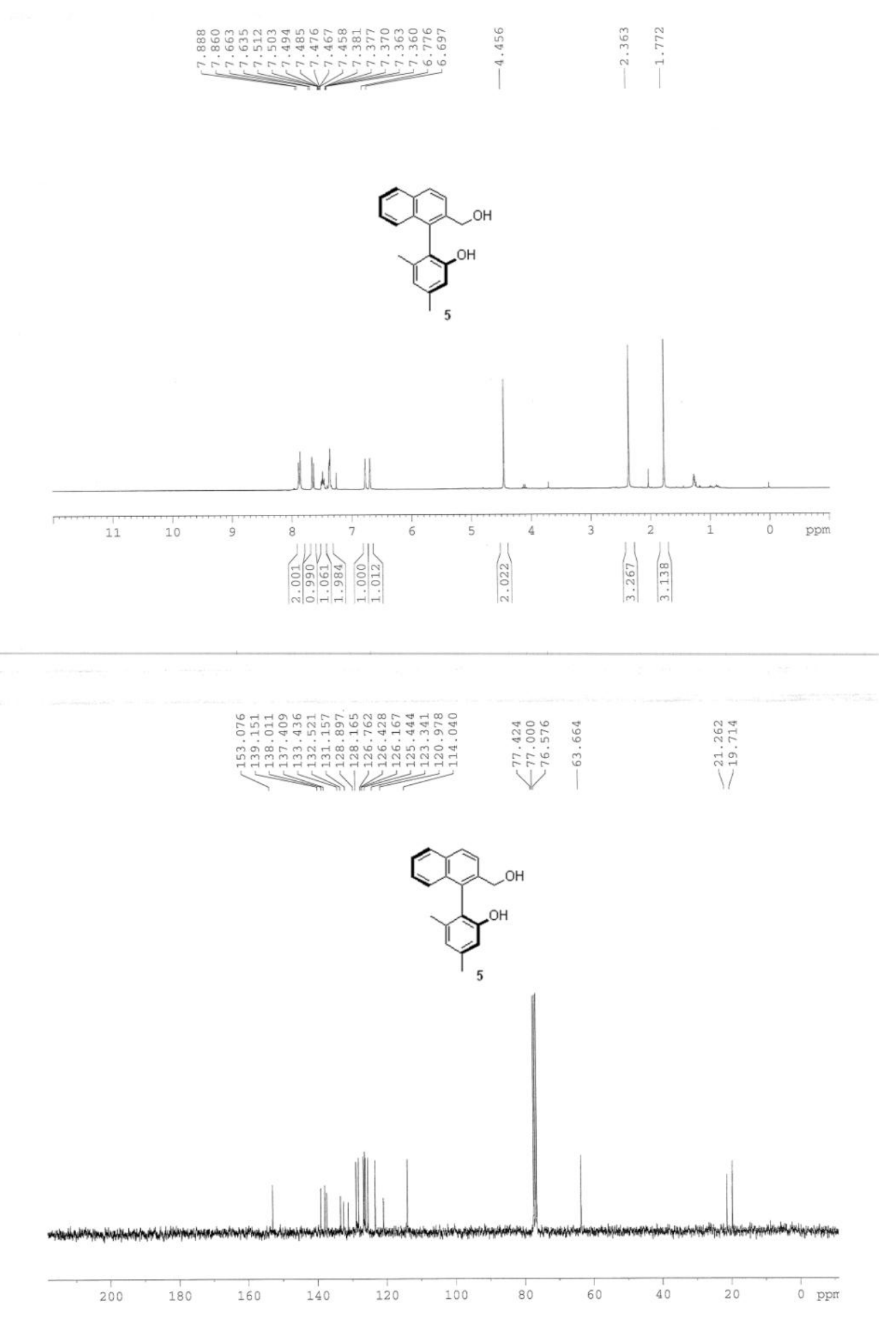




\section{University of New Mexico}

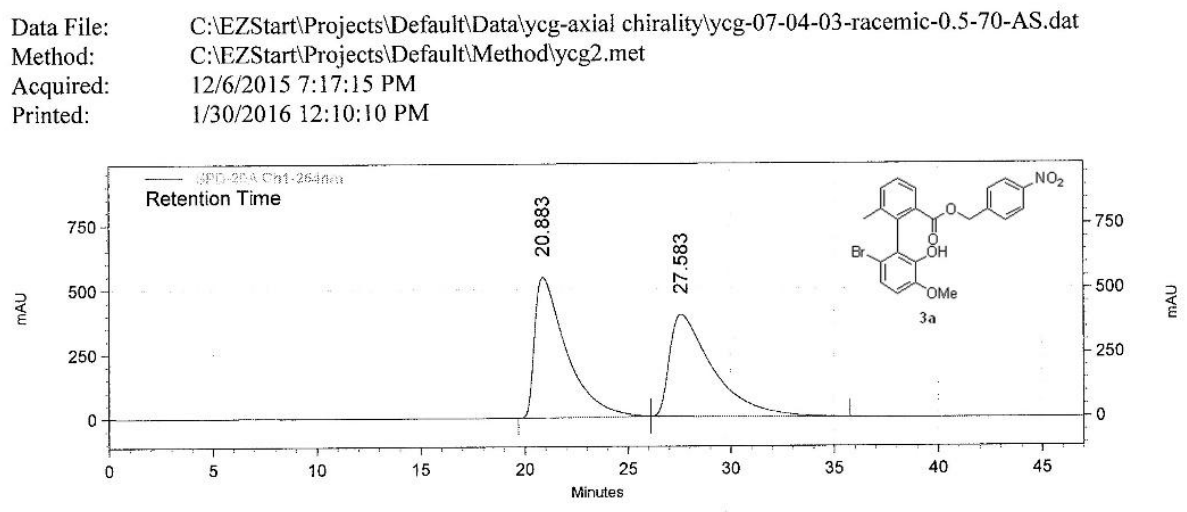

SPD-20A

\section{Ch1-254nm}

Results

Retention Time

20.883

27.583

Area

Area \%

$\begin{array}{rrr}50.41 & \text { Height } & \text { Height } \% \\ 49.59 & 546559 & 57.98 \\ & 396144 & 42.02\end{array}$

Totals

$\begin{array}{llll}57794347 & 49.59 & 396144 & 42.02\end{array}$

\begin{tabular}{|r|r|r|r|r|}
\hline Totals & 116539694 & 100.00 & 942703 & 100.00 \\
\hline
\end{tabular}

\section{University of New Mexico}

Data File: $\quad$ C: $\quad$ EZStart $\quad$ Projects $\backslash$ Default $\backslash$ Datalycg-axial chiralitylycg-07-40-1-AS-H-70-0.5.dat

Method: $\quad$ C:IEZStart $\backslash$ Projects $\backslash$ Default $\backslash$ Methodlycg2.met

Acquired: $\quad$ 12/17/2015 8:14:12 PM

Printed: $\quad$ 1/30/2016 12:09:36 PM

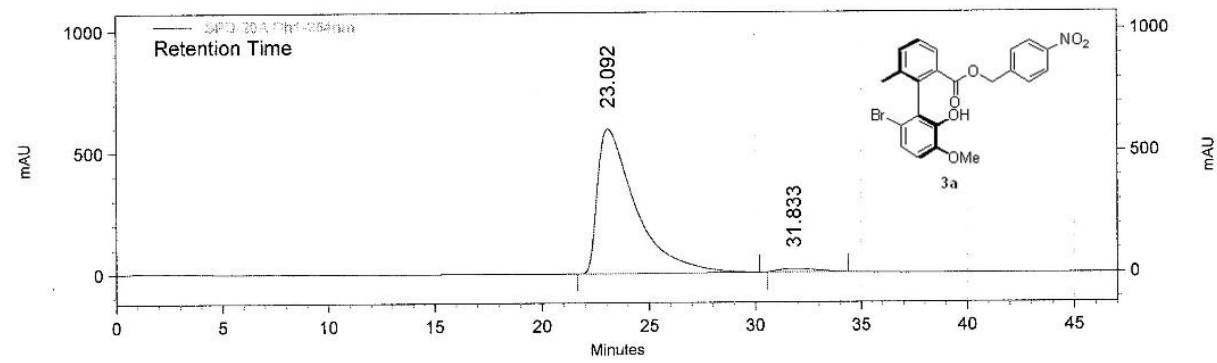

SPD-20A

Ch1-254nm

Results

Retention Time

23.092

31.833

Area

Area \%

\begin{tabular}{rrr}
97.80 & Height & Height \% \\
\hline & 596492 & 97.70
\end{tabular}

Totals

1663076

14062

2.30

\begin{tabular}{|r|r|r|r|r|}
\hline Totals & 75668378 & 100.00 & 610554 & 100.00 \\
\hline
\end{tabular}




\section{University of New Mexico}

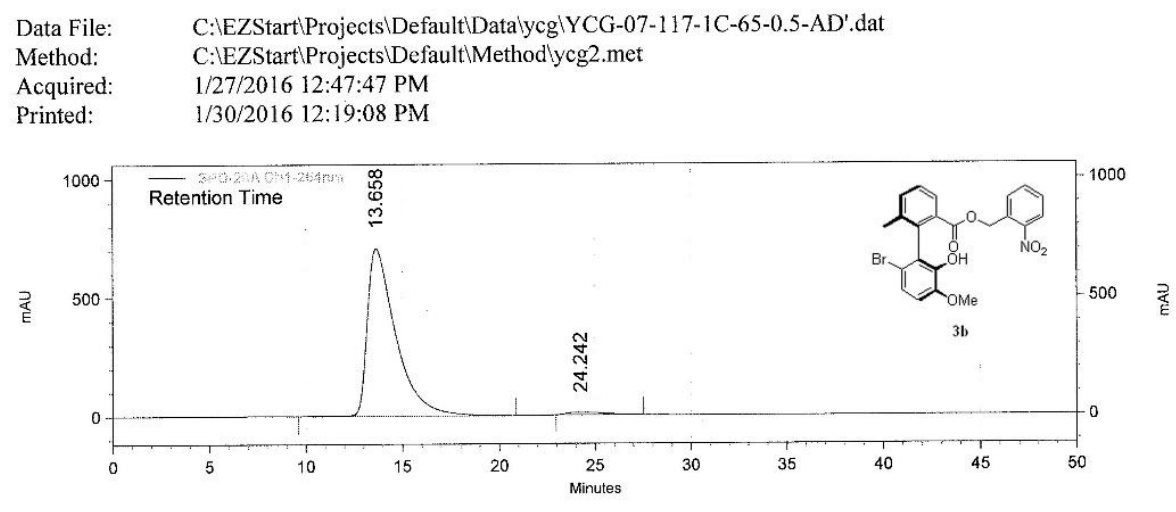

SPD-20A

Ch1-254nm

Results

Retention Time

\begin{tabular}{rrrrr} 
Time & Area & Area \% & Height & Height \% \\
\hline 13.658 & 72558487 & 98.15 & 706653 & 98.59 \\
24.242 & 1367683 & 1.85 & 10074 & 1.41
\end{tabular}

Totals

\begin{tabular}{|l|l|l|l|}
\hline 73926170 & 100.00 & 716727 & 100.00 \\
\hline
\end{tabular}

\section{University of New Mexico}

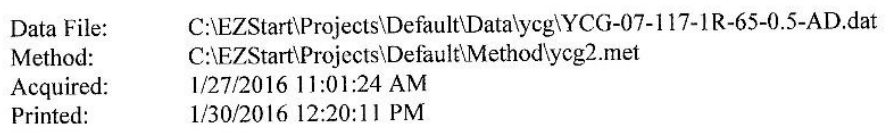

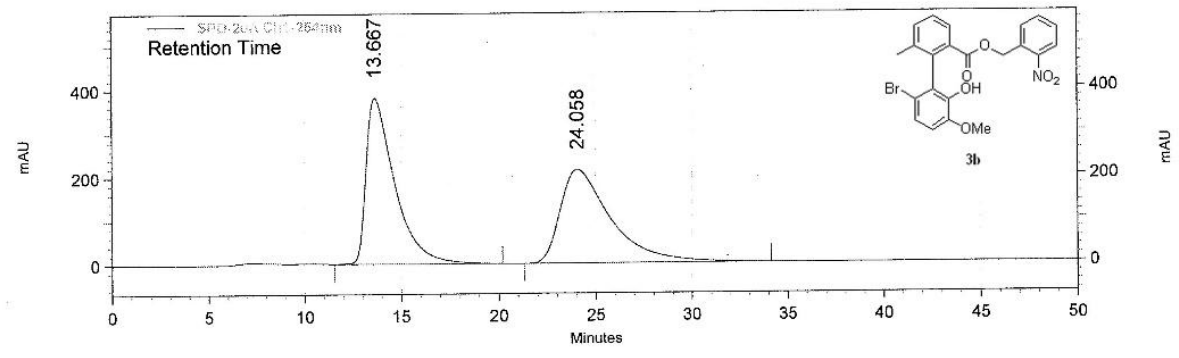

SPD-20A

Ch1-254nm

Results

Retention Time

13.667

Area

Area \%

$50.15 \quad 380492$

63.97

24.058

$38933501 \quad 49.85$

214345

36.03

\begin{tabular}{|r|r|r|r|r|}
\hline Totals & 78098281 & 100.00 & 594837 & 100.00 \\
\hline
\end{tabular}




\section{University of New Mexico}
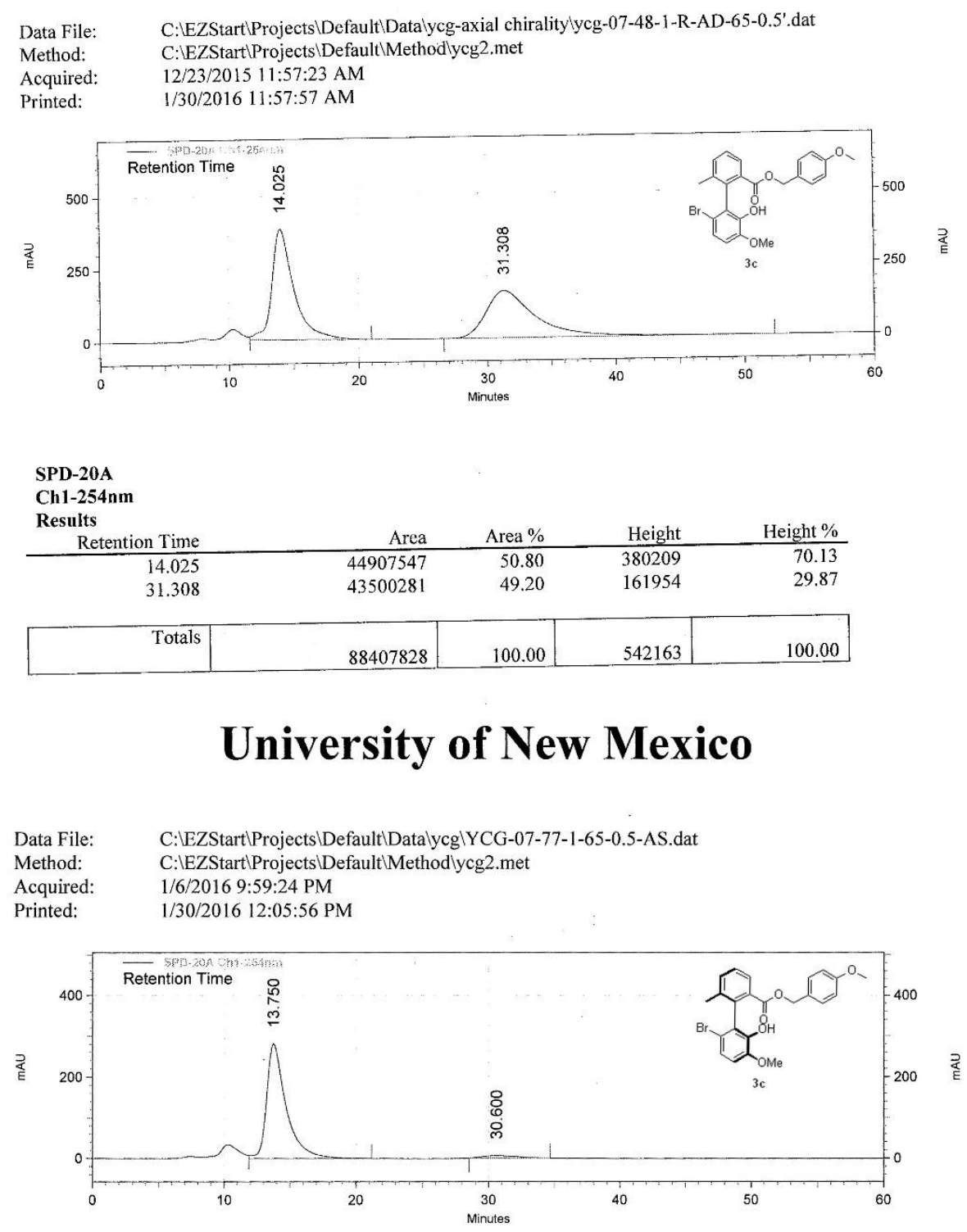

SPD-20A

Ch1-254nm

Results

Retention Time

13.750

\begin{tabular}{rrrr} 
Area & Area \% & Height & Height \% \\
\hline 29054176 & 96.45 & 280743 & 97.92 \\
1068294 & 3.55 & 5963 & 2.08
\end{tabular}

Totals

30122470

$100.00 \quad 286706$ 


\section{University of New Mexico}

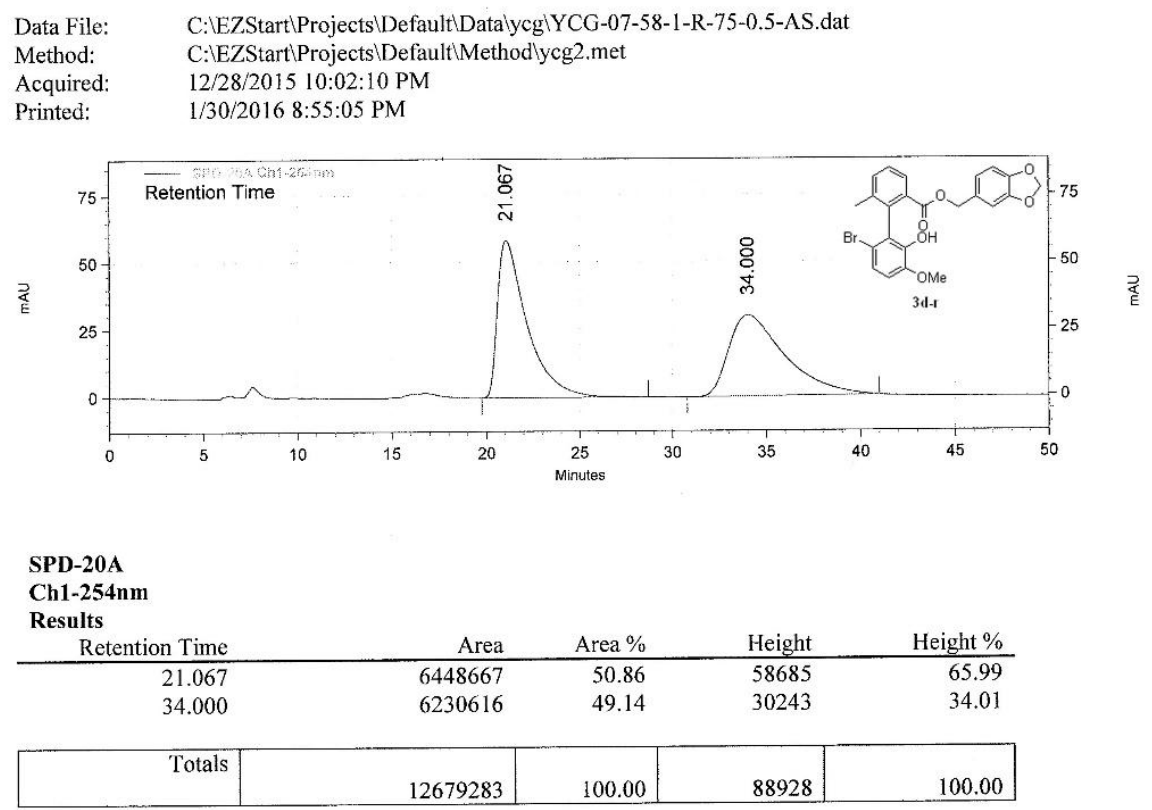

\section{University of New Mexico}

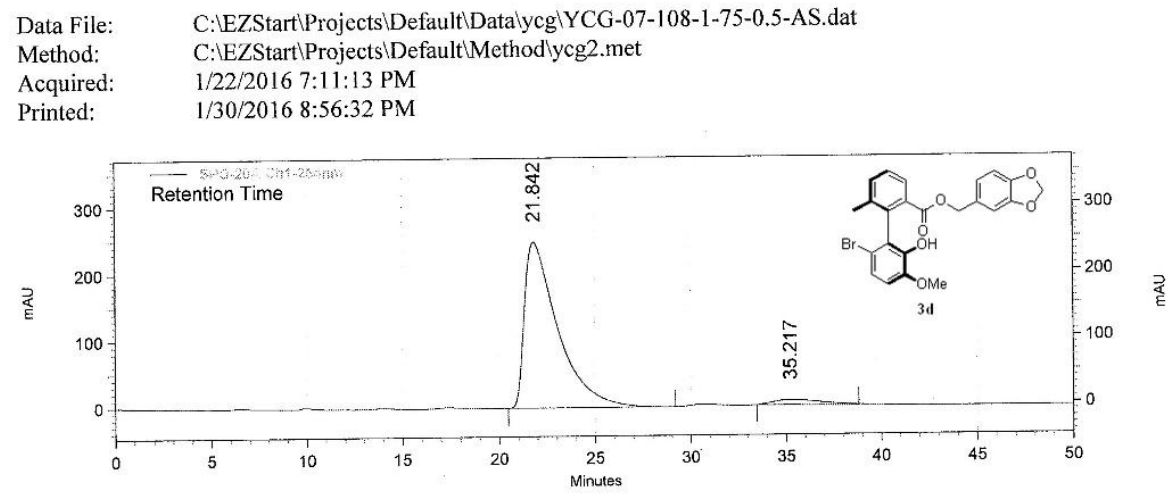

SPD-20A

Ch1-254nm

Results

Retention Time

21.842

35.217

Height

Height \%

\begin{tabular}{rrrr} 
Area & Area \% & Height & Height \% \\
\hline 30173262 & 95.57 & 250119 & 97.02 \\
1398345 & 4.43 & 7676 & 2.98
\end{tabular}

Totals

31571607

$100.00+257795$

100.00 


\section{University of New Mexico}

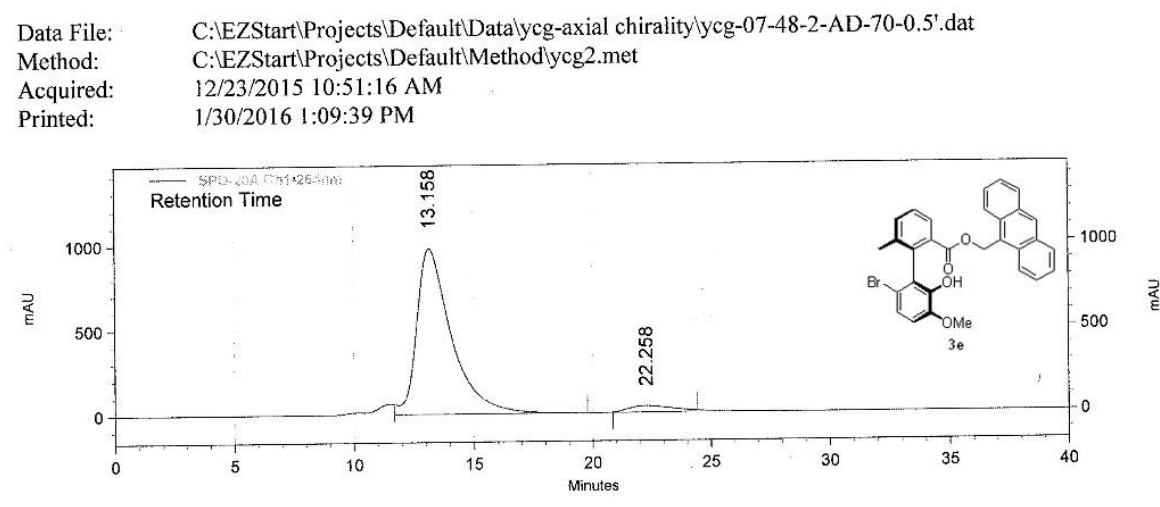

SPD-20A

Ch1-254nm

Results

Retention Time

13.158

\begin{tabular}{rrrr} 
Area & Area \% & Height & Height \% \\
\hline 9574347 & 95.29 & 973790 & 96.35 \\
8882769 & 4.71 & 36893 & 3.65
\end{tabular}

22.258

4882769

4.71

36893

3.65

Totals

103757116

$100.00 \quad 1010683$

100.00

\section{University of New Mexico}

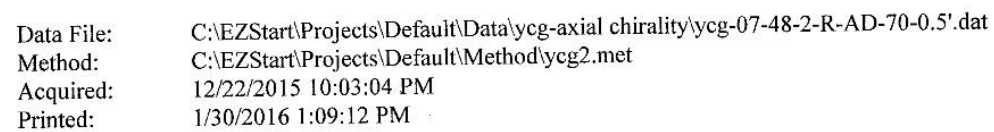

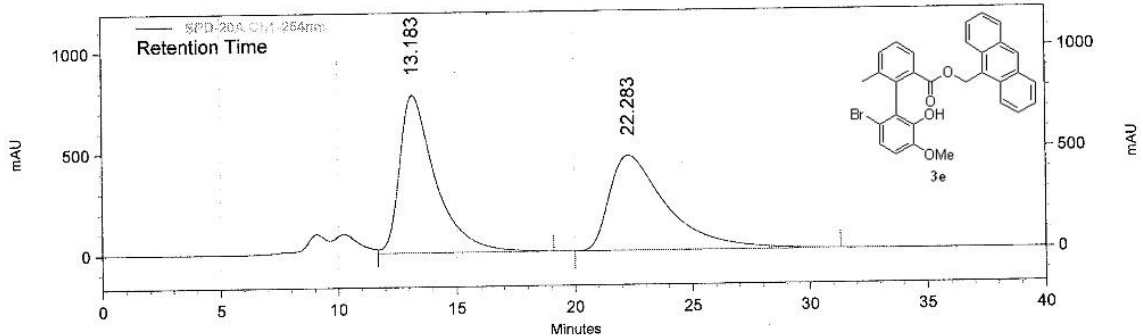

SPD-20A

Ch1-254nm

Results

Retention Time

Time

Area \% Height

Height \%

22.283

8194614

$49.16 \quad 46797$

37.52

Totals

161184641

100.00

1247192

100.00 


\section{University of New Mexico}
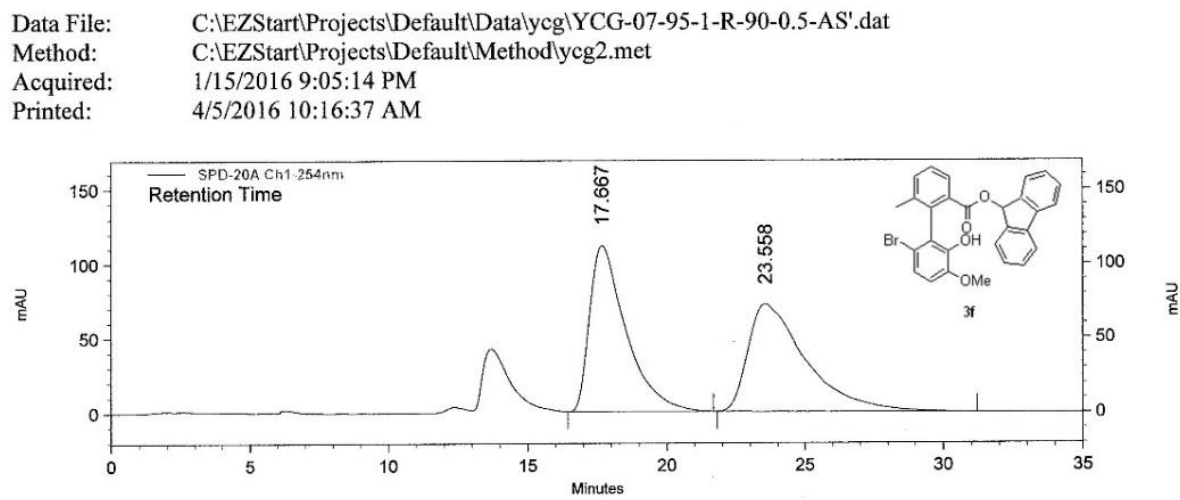

SPD-20A

Ch1-254nm

Results

Retention Time

17.667

23.558 Area \% Height $\quad$ Height \%

Total $\begin{array}{rrrr}10121543 & 49.23 & 111448 & 39.21\end{array}$

\begin{tabular}{|r|r|r|r|r|}
\hline Totals & 20560944 & 100.00 & 183346 & 100.00 \\
\hline
\end{tabular}

\section{University of New Mexico}

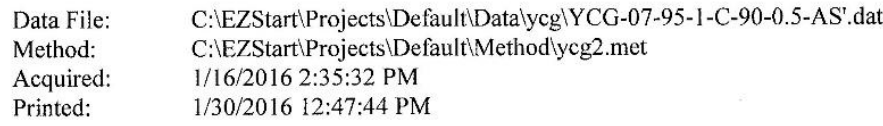

Data File: $\quad$ C: $\quad$ EZStart $\backslash$ Projects $\backslash$ Default $\backslash$ Datalycg $\backslash Y C G-07-95-1-C-90-0.5-A S '$ dat

Method: $\quad$ C:\EZStart $\quad$ Projects $\backslash$ Default $\backslash$ Methodlycg2.met

Acquired: $\quad$ 1/16/2016 2:35:32 PM

Printed: $\quad 1 / 30 / 201612: 47: 44$ PM

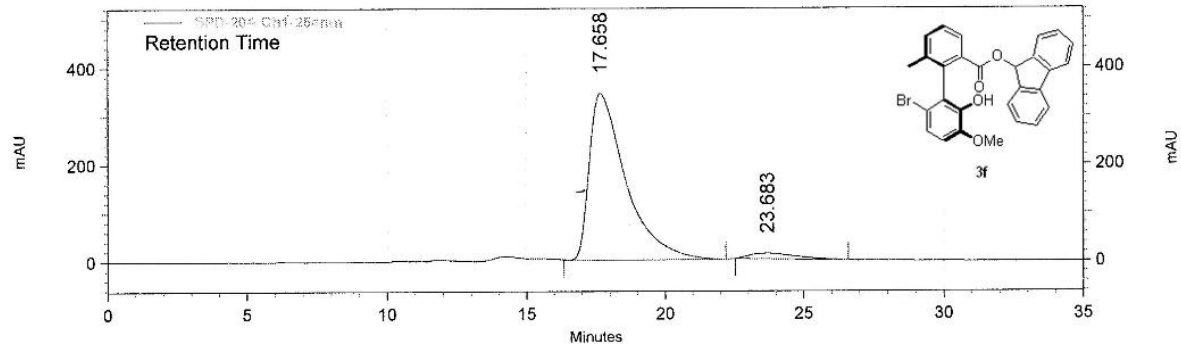

SPD-20A

Ch1-254nm

Results

Retention Time

17.658

Area

Area \%

$\begin{array}{rrr}95.91 & 344317 & 96.70 \\ 4.09 & 11756 & 3.30\end{array}$

Totals $\begin{array}{r}1352257 \\ \hline\end{array}$

11756

3.30

\begin{tabular}{|r|r|r|r|r|}
\hline Totals & 33024125 & 100.00 & 356073 & 100.00 \\
\hline
\end{tabular}




\section{University of New Mexico}

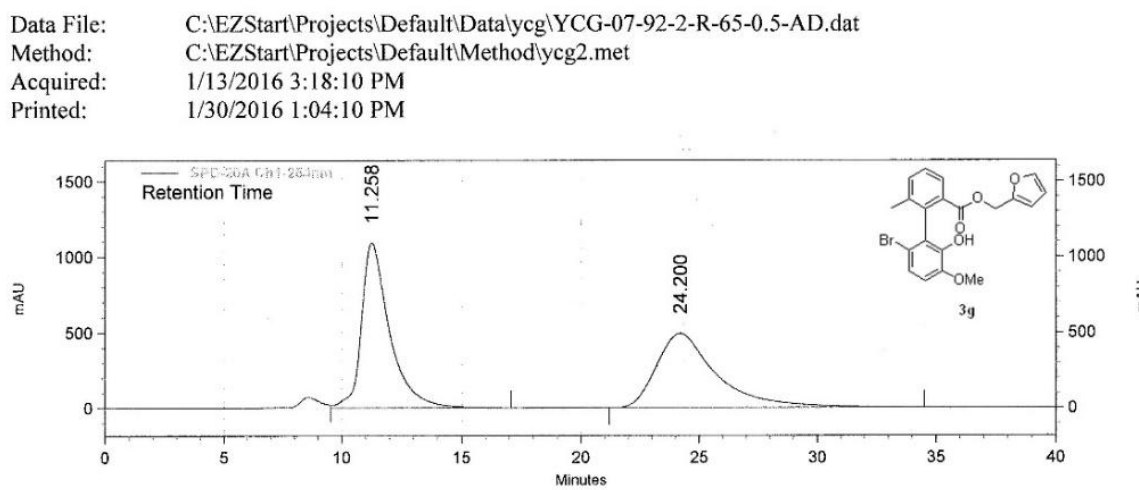

SPD-20A

Ch1-254nm

Results

Retention Time

11.25

Area Area \%

\begin{tabular}{rrrr} 
Area & Area \% & Height & Height \% \\
\hline 89488489 & 50.70 & 1089742 & 68.93 \\
87031976 & 49.30 & 491298 & 31.07
\end{tabular}

Totals

\begin{tabular}{|r|r|r|r|r|}
\hline Totals & 176520465 & 100.00 & 1581040 & 100.00 \\
\hline
\end{tabular}

\section{University of New Mexico}
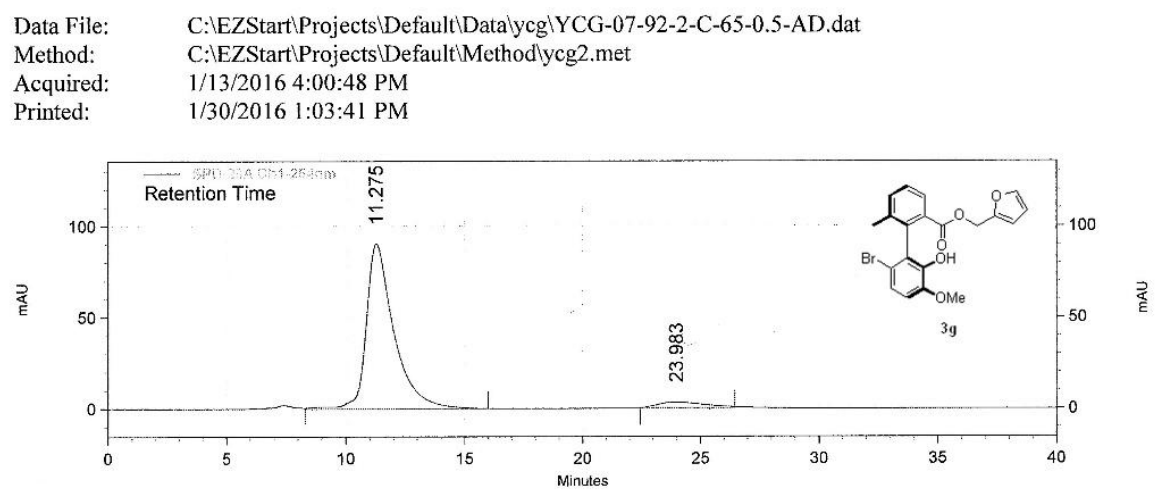

SPD-20A

Ch1-254nm

Results

Retention Time

11.275

23.983

Area

Area \%

Height

$90293 \quad 96.74$

Total

$\begin{array}{lr}403158 & 5.21\end{array}$

$3045 \quad 3.26$

\begin{tabular}{|r|r|r|r|r|}
\hline Totals & 7744780 & 100.00 & 93338 & 100.00 \\
\hline
\end{tabular}




\section{University of New Mexico}

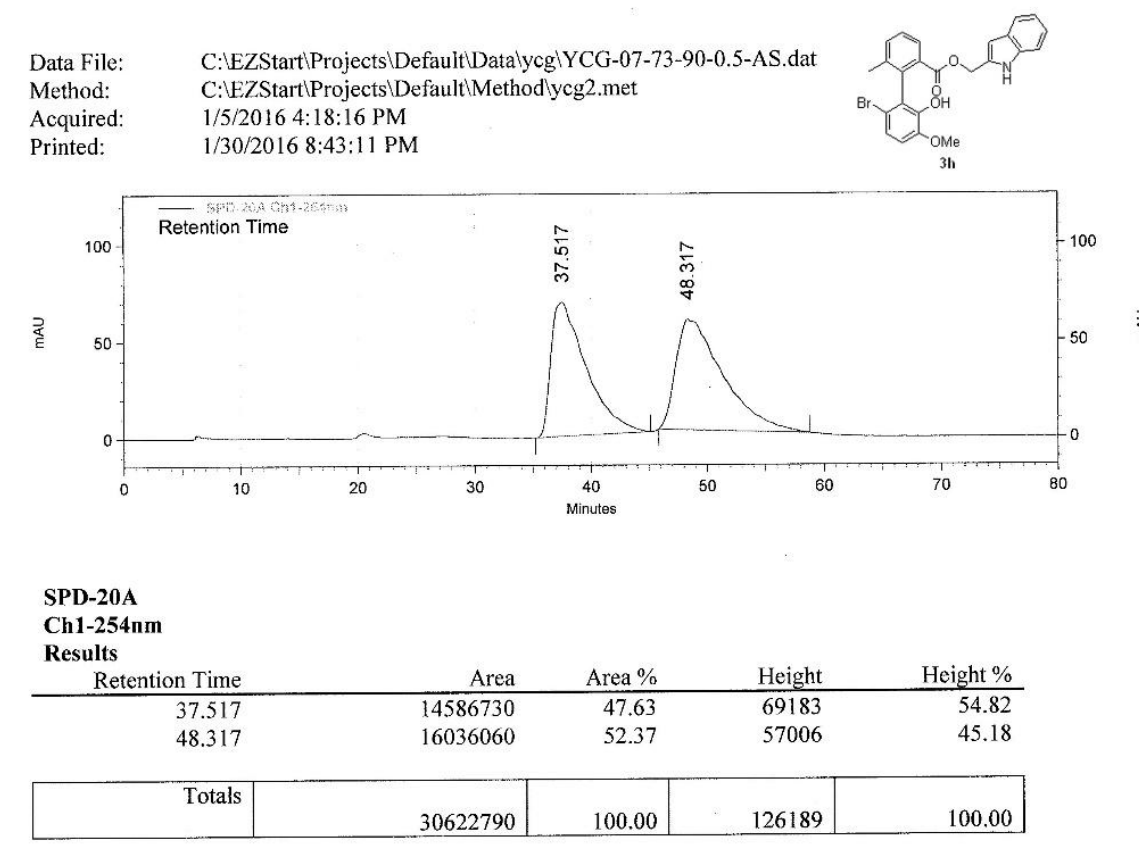

\section{University of New Mexico}

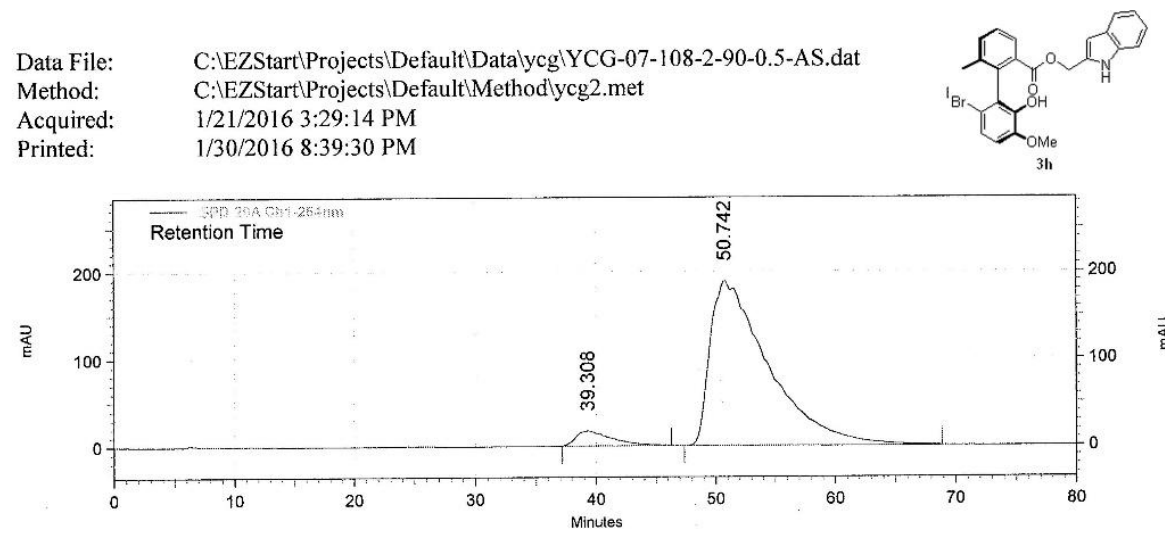

SPD-20A

\section{Ch1-254nm}

Results

Retention Time

39.308

50.742

Area
3532387
64069963

Area \%

Height

Height \%

Totals

94.77

17120
188485

\begin{tabular}{|r|r|r|r|r|}
\hline Totals & 67602350 & 100.00 & 205605 & 100.00 \\
\hline
\end{tabular}




\section{University of New Mexico}

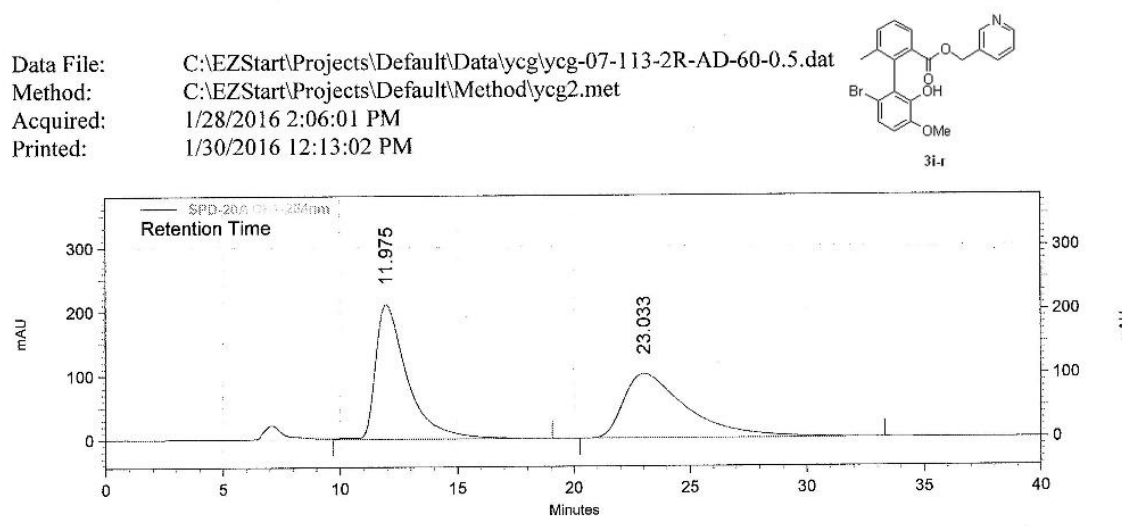

SPD-20A

Ch1-254nm

Results

Retention Time

11.975

23.033

Area \%

Totals

$49.12-100587$

$\begin{array}{ll}100587 & 32.36\end{array}$

\begin{tabular}{|r|r|r|r|r|}
\hline Totals & 39011452 & 100.00 & 310828 & 100.00 \\
\hline
\end{tabular}

\section{University of New Mexico}

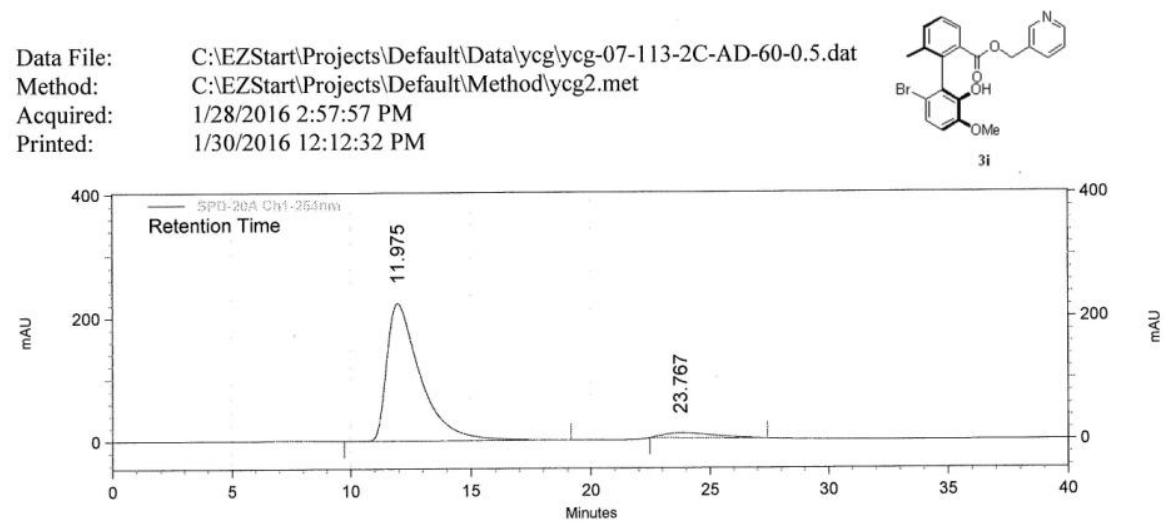

SPD-20A

Ch1-254nm

Results

Retention Time

11.975

Area \%

Height \%

23.767

1171974

5.86

222805

7731

Totals

22816462

$100.00 \quad 230536$

100.00 


\section{University of New Mexico}

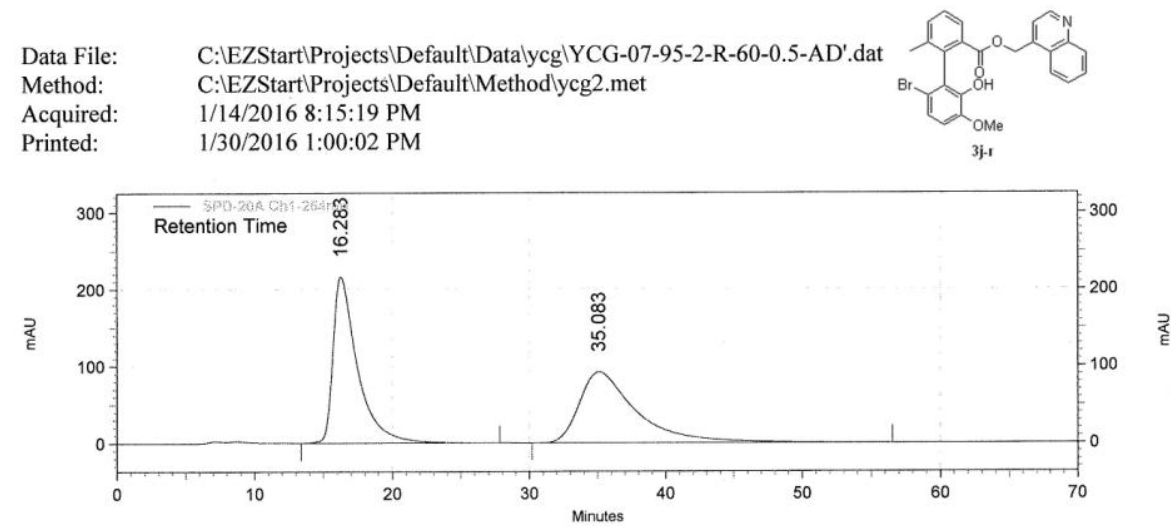

SPD-20A

Ch1-254nm

Results

\begin{tabular}{rrrrrr|}
$\begin{array}{r}\text { Retention Time } \\
\text { Area }\end{array}$ & Area \% & Height & Height \% \\
\hline 16.283 & 26733847 & 50.15 & 216138 & 70.12 \\
35.083 & 26569331 & 49.85 & 92115 & 29.88 \\
\multicolumn{2}{|c|}{ Totals } & 53303178 & 100.00 & 308253 & 100.00 \\
\hline
\end{tabular}

\section{University of New Mexico}

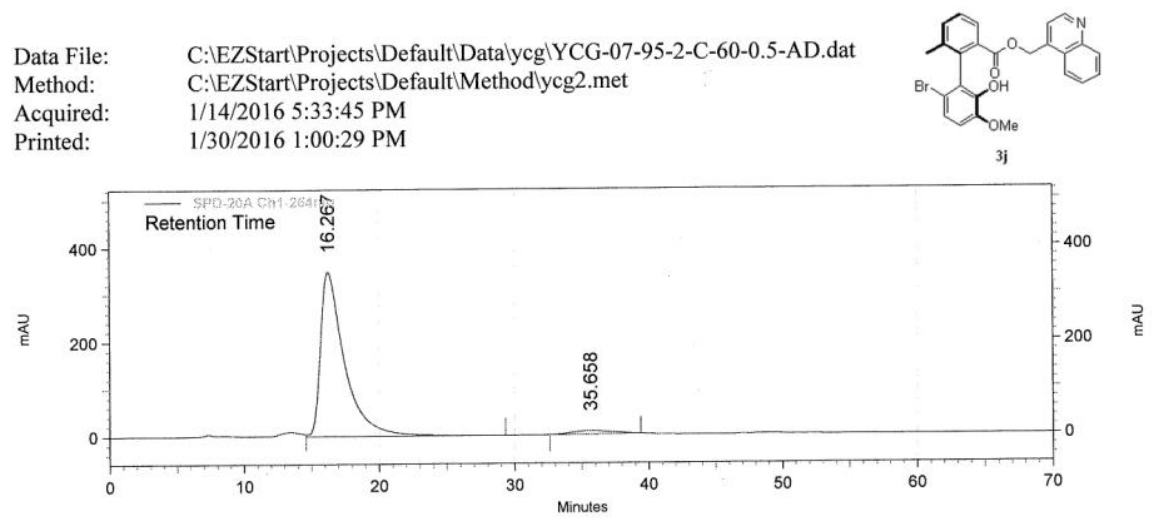

SPD-20A

Ch1-254nm

Results

Retention Tim

16.267

35.658

Ar
431952
15002

Area \%

Heigh

\begin{tabular}{rr} 
Height & Height \% \\
\hline 348059 & 97.93
\end{tabular}

Totals

44695416

100.00

355410

100.00 


\section{University of New Mexico}

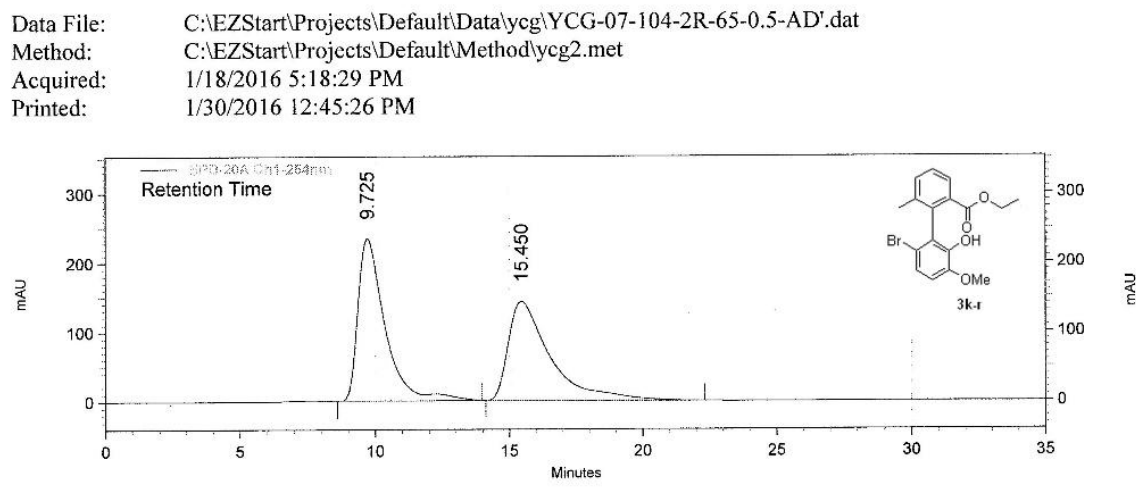

SPD-20A

Ch1-254nm

Results

Retention Time

9.725

\begin{tabular}{rrrr} 
Area & Area \% & Height & Height \% \\
\hline 149656 & 50.98 & 233793 & 62.21 \\
525926 & 49.02 & 142007 & 37.79
\end{tabular}

\begin{tabular}{|r|r|r|r|r|}
\hline Totals & 31675582 & 100.00 & 375800 & 100.00 \\
\hline
\end{tabular}

\section{University of New Mexico}

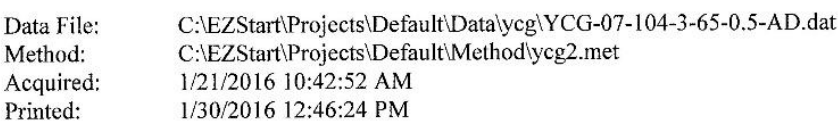

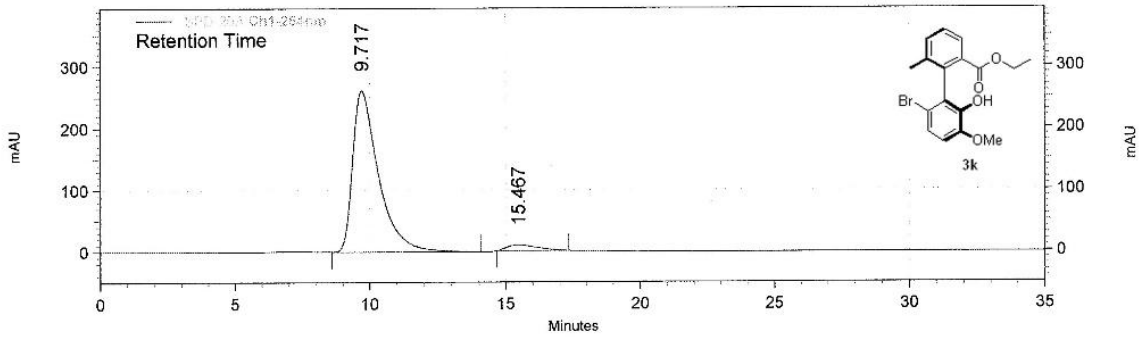

SPD-20A

Ch1-254nm

Results

Retention Time

\begin{tabular}{rrrrr} 
Trime & Area & Area \% & Height & Height \% \\
\hline 9.717 & 17397164 & 95.97 & 261369 & 96.56 \\
15.467 & 730592 & 4.03 & 9298 & 3.44
\end{tabular}

Totals

\begin{tabular}{|l|r|r|r|}
\hline 18127756 & 100.00 & 270667 & 100.00 \\
\hline
\end{tabular}




\section{University of New Mexico}

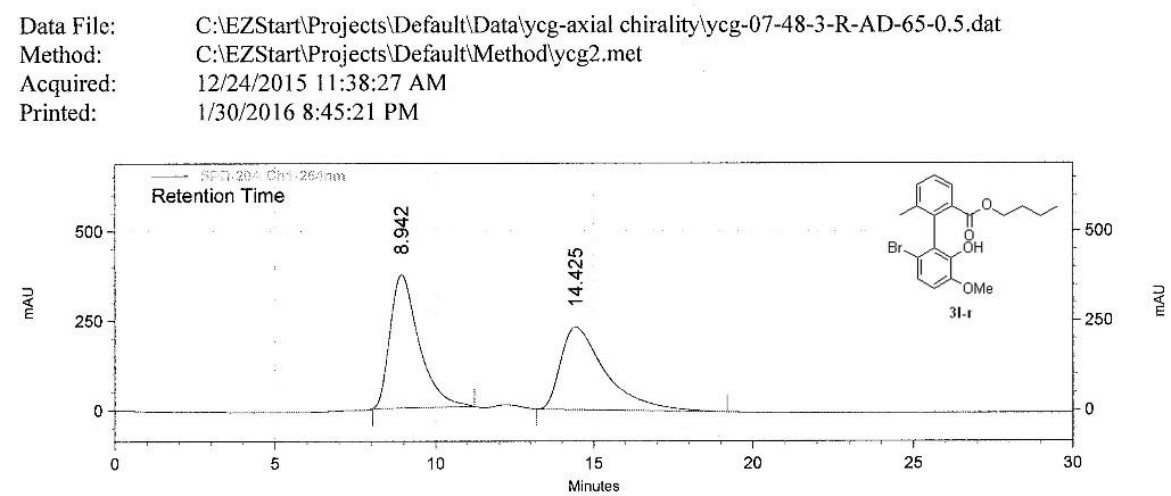

SPD-20A

Ch1-254nm

Results

Retention Time

\begin{tabular}{rrrrr} 
Time & Area & Area \% & Height & Height \% \\
\hline 8.942 & 23279131 & 50.72 & 372648 & 61.78 \\
14.425 & 22620158 & 49.28 & 230538 & 38.22
\end{tabular}

\begin{tabular}{|r|r|r|r|r|}
\hline Totals & 45899289 & 100.00 & 603186 & 100.00 \\
\hline
\end{tabular}

\section{University of New Mexico}

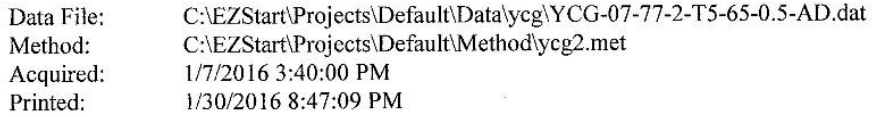

Data File: $\quad$ C:IEZStart $\quad$ Projects $\backslash$ Default $\backslash$ Datalycg $\backslash Y C G-07-77-2-T 5-65-0.5-A D$ dat

Method: C:IEZStart $\quad$ Projects\Default Methodlycg2.met

Acquired: $\quad$ 1/7/2016 3:40:00 PM

Printed: $\quad$ 1/30/2016 8:47:09 PM

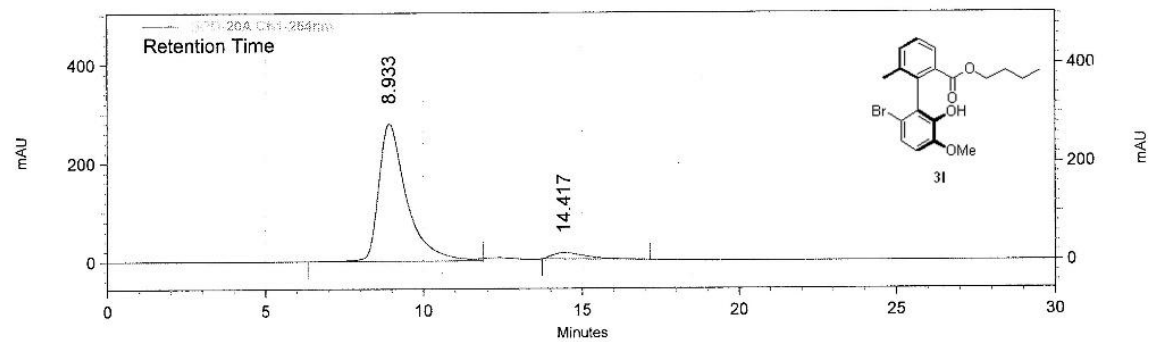

SPD-20A

Ch1-254nm

Results

Retention Time

8.933

Area $\quad$ Area $\%$

Height $\quad$ Height \%

14.417

$\begin{array}{rrrr}17150407 & 94.87 & 279266 & 95.77 \\ 926836 & 5.13 & 12334 & 4.23\end{array}$

Totals

\begin{tabular}{l|l|r|}
\hline 18077243 & 100.00 & 291600 \\
\hline
\end{tabular}

100.00 


\section{University of New Mexico}

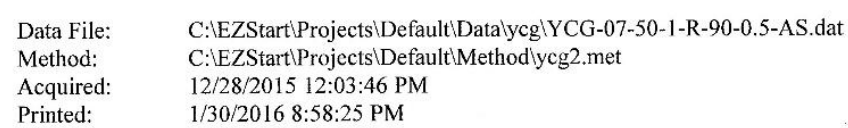

Printed: 1/30/2016 8:58:25 PM

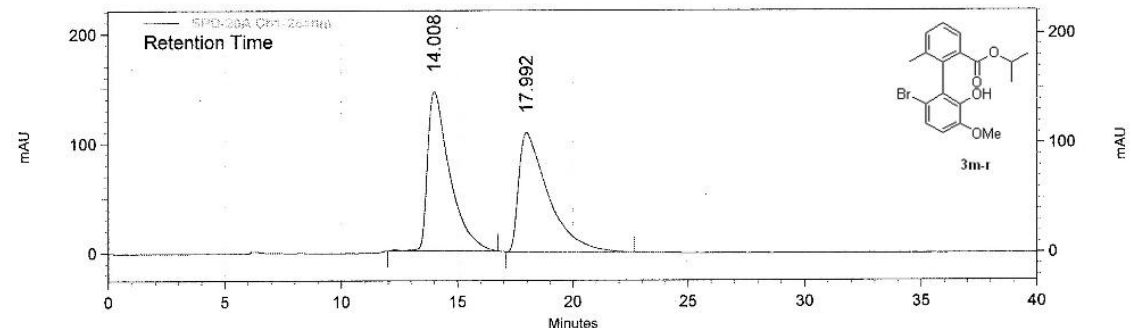

SPD-20A

Ch1-254nm

Results

Retention Time

14.008

17.992

\begin{tabular}{rrrr} 
Area & Area \% & Height & Height \% \\
\hline 9704040 & 50.00 & 145221 & 57.22 \\
9705133 & 50.00 & 108590 & 42.78
\end{tabular}

Totals

\begin{tabular}{l|l}
100.00 & 253811 \\
\hline
\end{tabular}

\section{University of New Mexico}

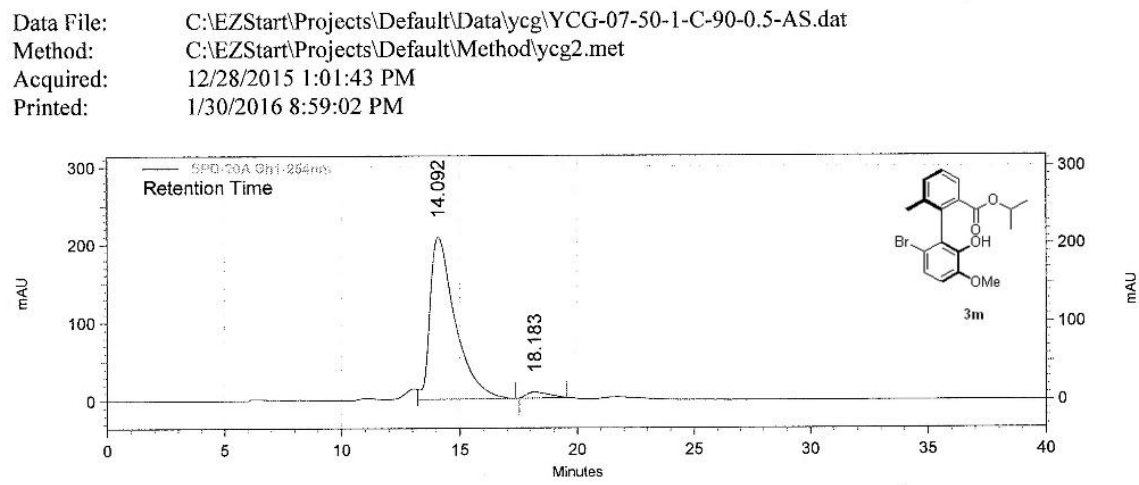

SPD-20A

Ch1-254nm

Results

Retention Time

14.092

18.183

Area \%

\begin{tabular}{rrr} 
Heaight & Height \% \\
\hline 6.76 & 207548 & 96.48 \\
3.24 & 7572 & 3.52
\end{tabular}

Totals

Area
15352024

513545

$100.00 \quad 215120 \mid$

100.00 


\section{University of New Mexico}

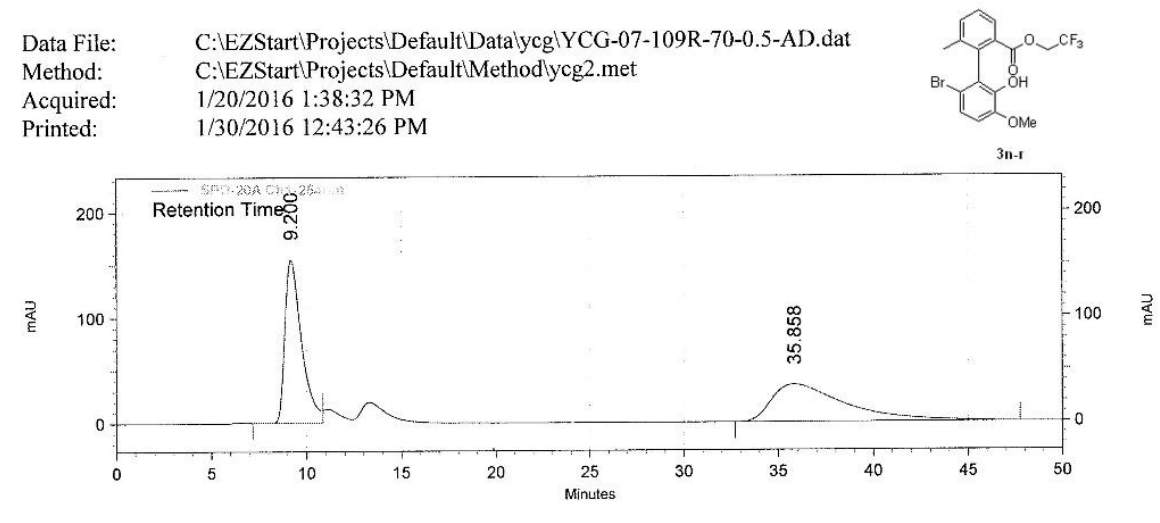

\section{SPD-20A \\ Ch1-254nm \\ Results}

Retention Time

9.200

35.858

\begin{tabular}{rrrr} 
Area & Area \% & Height & Height \% \\
\hline 9441330 & 49.98 & 154960 & 81.39 \\
9448513 & 50.02 & 35442 & 18.61
\end{tabular}

Totals

\begin{tabular}{|r|r|r|r|r|}
\hline Totals & 18889843 & 100.00 & 190402 & 100.00 \\
\hline
\end{tabular}

\section{University of New Mexico}

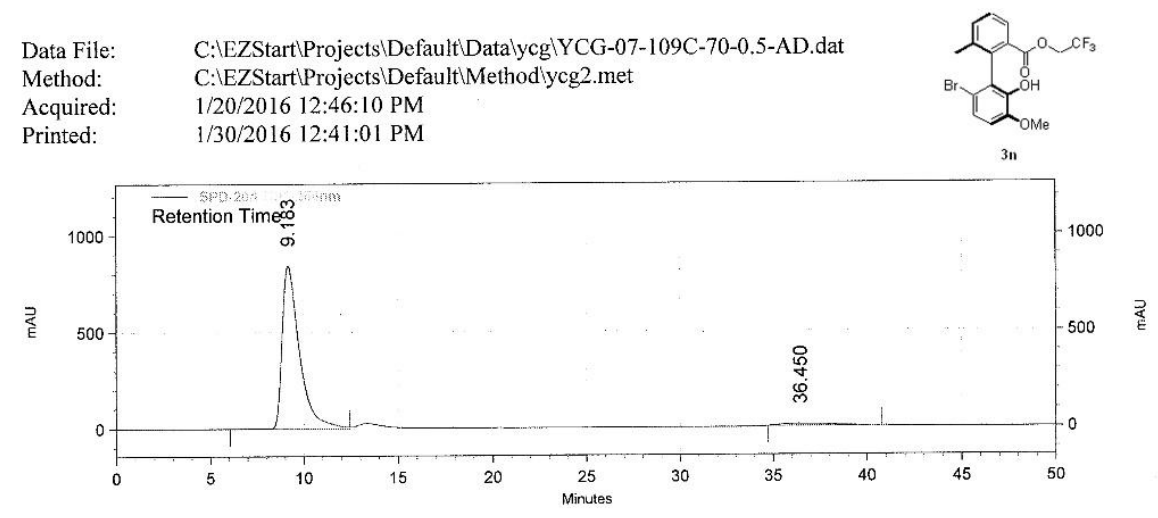

SPD-20A

Ch1-254nm

Results

Retention Time

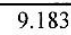

9.183
36.450

\begin{tabular}{rrrr} 
Area & Area \% & Height & Height \% \\
\hline 54544661 & 96.38 & 843775 & 98.75 \\
2051363 & 3.62 & 10693 & 1.25
\end{tabular}

Totals

\begin{tabular}{|l|r|r|r|}
\hline 56596024 & 100.00 & 854468 & 100.00 \\
\hline
\end{tabular}




\section{University of New Mexico}

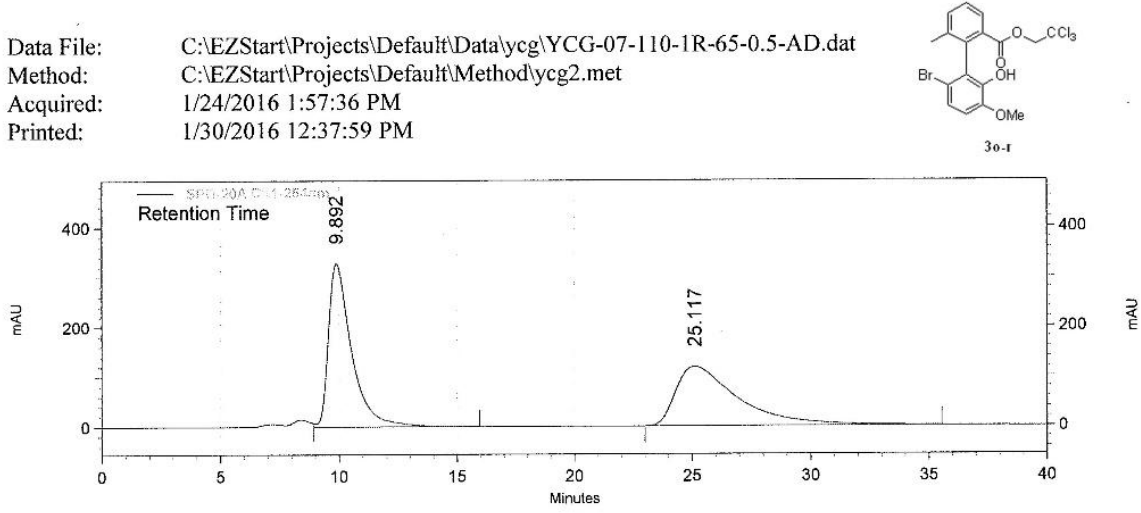

SPD-20A

Ch1-254nm

Results

Retention Time

9.892

\begin{tabular}{rrrr} 
Area & Area \% & Height & Height \% \\
\hline 2482223 & 50.83 & 328064 & 73.43 \\
1750787 & 49.17 & 118731 & 26.57
\end{tabular}

\begin{tabular}{|r|r|r|r|r|}
\hline Totals & 44233010 & 100.00 & 446795 & 100.00 \\
\hline
\end{tabular}

\section{University of New Mexico}

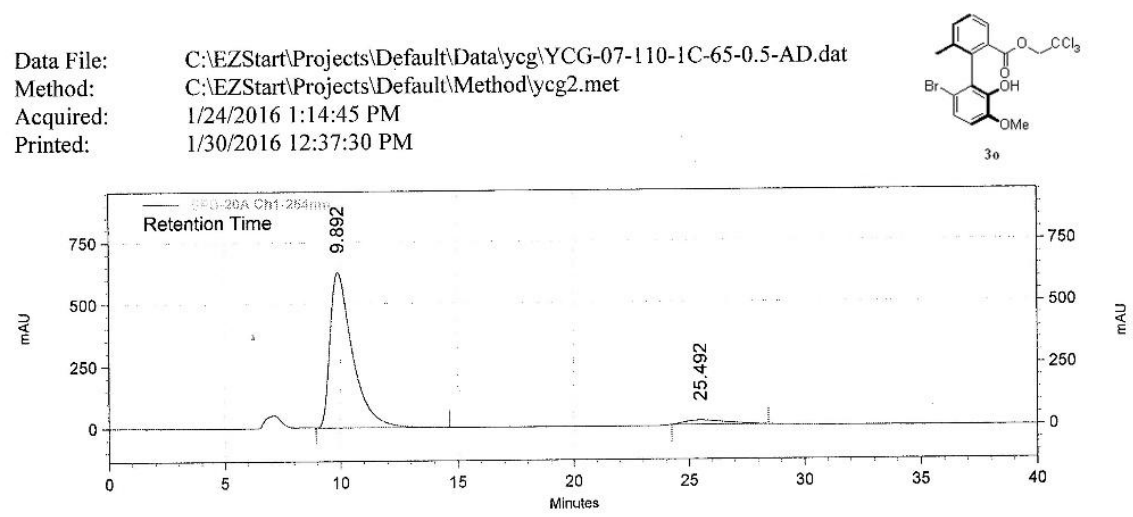

SPD-20A

Ch1-254nm

Results

Retention Time

\begin{tabular}{rrrrr} 
Time & Area & Area \% & Height & Height \% \\
\hline 9.892 & 42205248 & 95.08 & 627231 & 97.39 \\
25.492 & 2184074 & 4.92 & 16805 & 2.61
\end{tabular}

Total

2.61

\begin{tabular}{|r|r|r|r|r|}
\hline Totals & 44389322 & 100.00 & 644036 & 100.00 \\
\hline
\end{tabular}




\section{University of New Mexico}

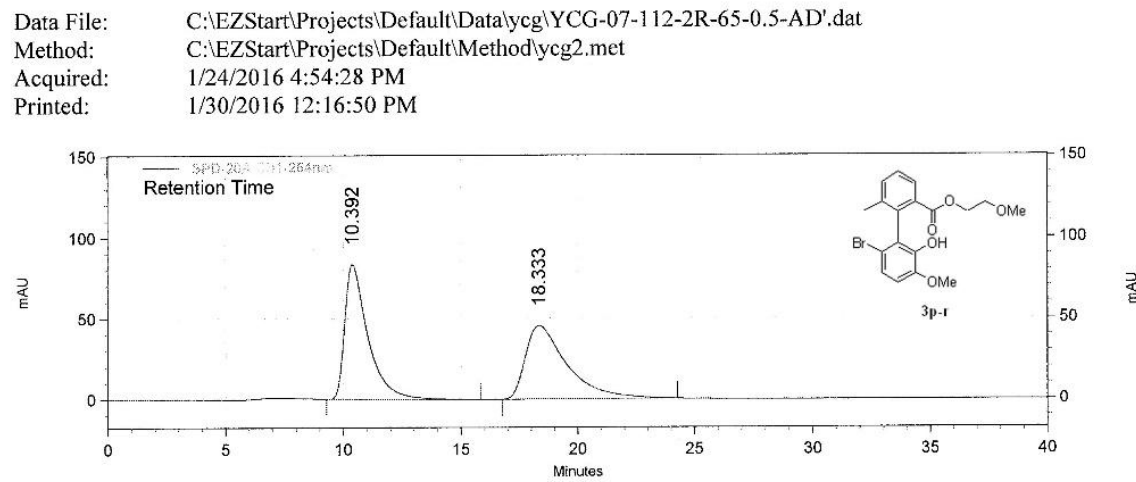

SPD-20A

Ch1-254nm

Results

Retention Time

10.392

Area Area \%

$\begin{array}{llll}5674406 & 50.36 & 83320 & 64.68\end{array}$

18.333

5592805

$\begin{array}{ll}50.36 & 83320 \\ & 45490\end{array}$

35.32

\begin{tabular}{|r|r|r|r|r|}
\hline Totals & 11267211 & 100.00 & 128810 & $\mathrm{I} 00.00$ \\
\hline
\end{tabular}

\section{University of New Mexico}

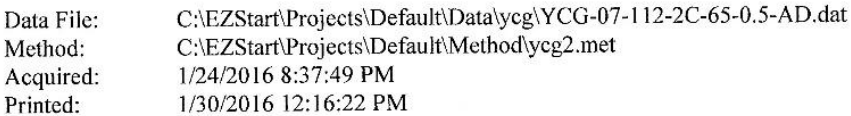

Data File: $\quad$ C:\EZStart Projects $\backslash$ Default $\backslash D$ atalycg $\backslash Y C G-07-112-2 C-65-0.5-A D$ dat

Method: $\quad$ C:IEZStartProjects $\backslash$ Default $\backslash M$ ethodlycg2.met

Acquired: $\quad$ 1/24/2016 8:37:49 PM

Printed: $\quad$ 1/30/2016 12:16:22 PM

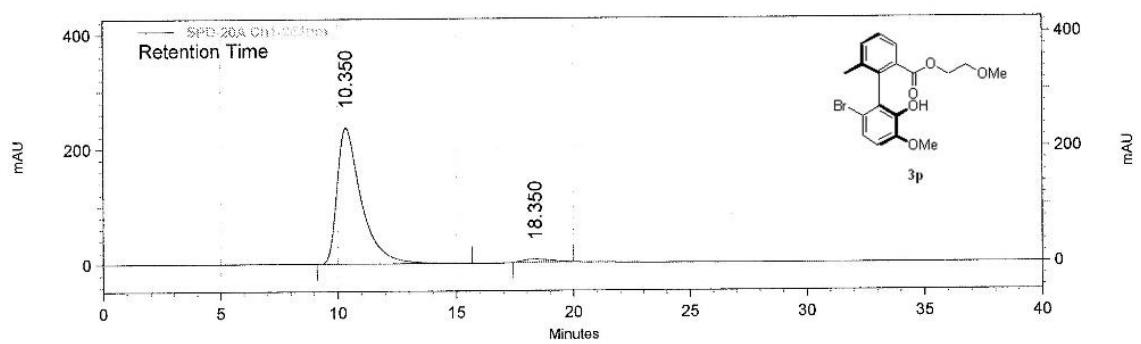

SPD-20A

Ch1-254nm

Results

Retention Time

10.350

Area

Area \%

$97.52 \quad 236560 \quad 97.86$

18.350

428672

2.48

516

97.86

Totals

17260211

$100.00 \quad 241721$

100.00 


\section{University of New Mexico}

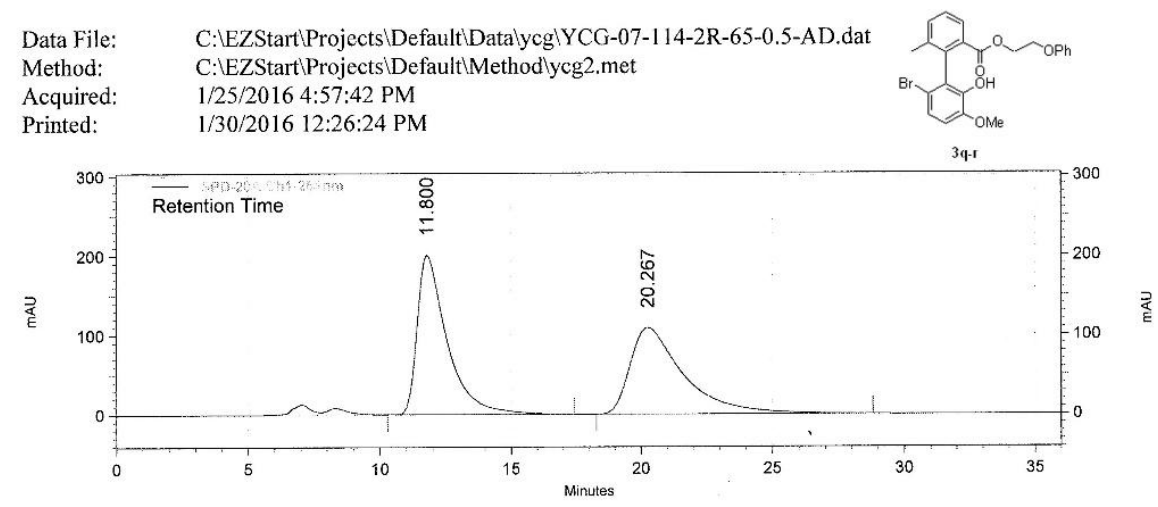

SPD-20A

Ch1-254nm

Results

\begin{tabular}{rrrrrr} 
Retention Time & Area & Area \% & Height & Height \% \\
\hline 11.800 & 15840167 & 50.72 & 199919 & 64.79 \\
20.267 & 15389332 & 49.28 & 108655 & 35.21 \\
\multicolumn{2}{|c|}{ Totals } & 31229499 & 100.00 & 308574 & 100.00 \\
\hline
\end{tabular}

\section{University of New Mexico}

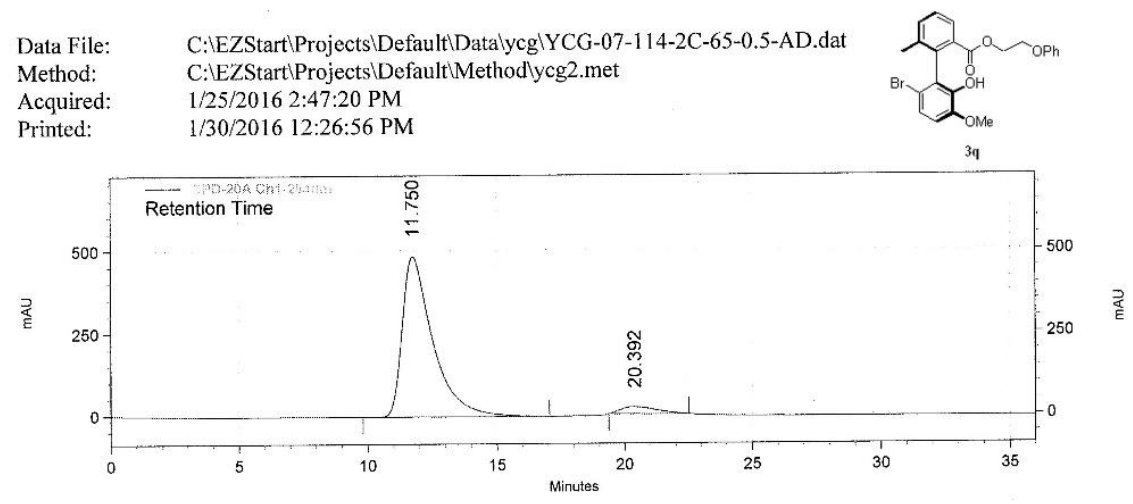

SPD-20A

Ch1-254nm

Results

Retention Time

11.750

20.392

\begin{tabular}{rrrr} 
Area & Area \% & Height & Height \% \\
\hline 40013448 & 95.17 & 486386 & 95.89 \\
2029368 & 4.83 & 20836 & 4.11
\end{tabular}

Totals

507222

100.00 


\section{University of New Mexico}
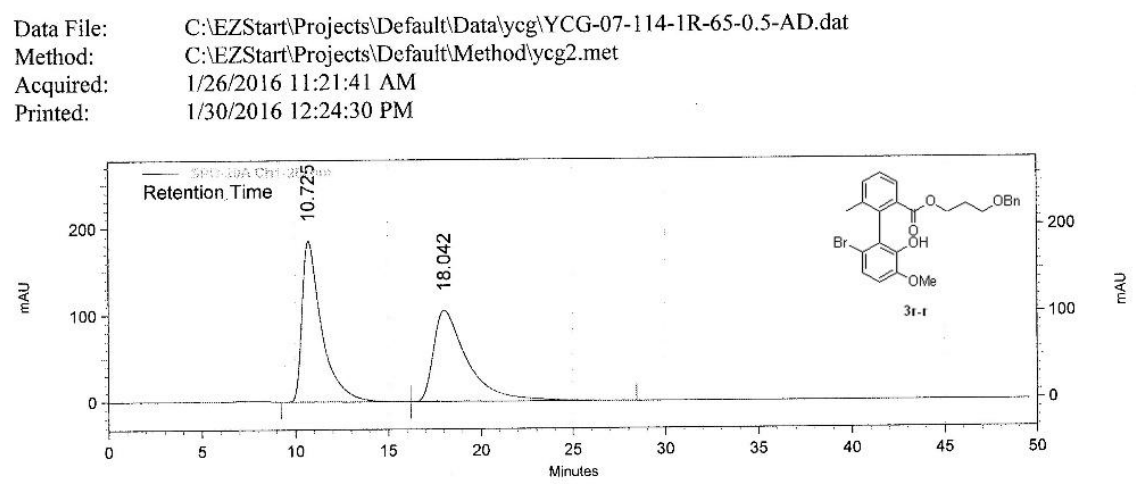

\section{SPD-20A}

Ch1-254nm

Results

\begin{tabular}{rrrrr} 
Retention Time & Area & Area \% & Height & Height \% \\
\hline 10.725 & 13606802 & 50.46 & 185472 & 63.79 \\
18.042 & 13359522 & 49.54 & 105278 & 36.21
\end{tabular}

\begin{tabular}{|r|r|r|r|r|}
\hline Totals & 26966324 & 100.00 & 290750 & 100.00 \\
\hline
\end{tabular}

\section{University of New Mexico}
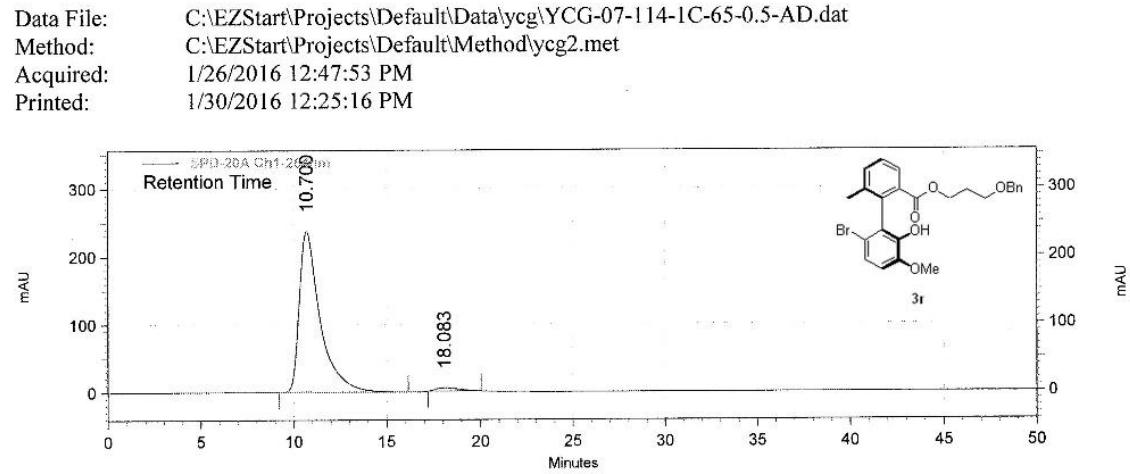

SPD-20A

Ch1-254nm

Results

Retention Time

\begin{tabular}{lrrrr} 
Time & Area & Area \% & Height & Height \% \\
\hline 10.700 & 18438998 & 97.77 & 236630 & 98.04 \\
18.083 & 419922 & 2.23 & 4719 & 1.96
\end{tabular}

419922

T

241349

100.00 


\section{University of New Mexico}

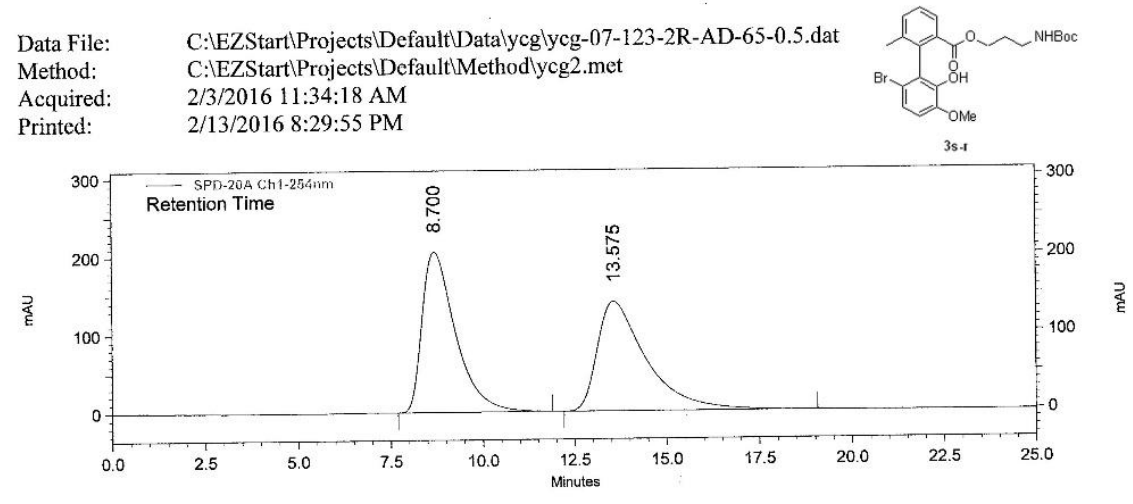

SPD-20A

Ch1-254nm

Results

Retention Time

8.700

13.575

Area \%

\begin{tabular}{rrr} 
\% $\%$ & Height & Height \% \\
\hline 0.20 & 206016 & 59.45 \\
9.80 & 140521 & 40.55
\end{tabular}

Totals

\begin{tabular}{|l|l|l|}
\hline 100.00 & 346537 & 100.00 \\
\hline
\end{tabular}

\section{University of New Mexico}

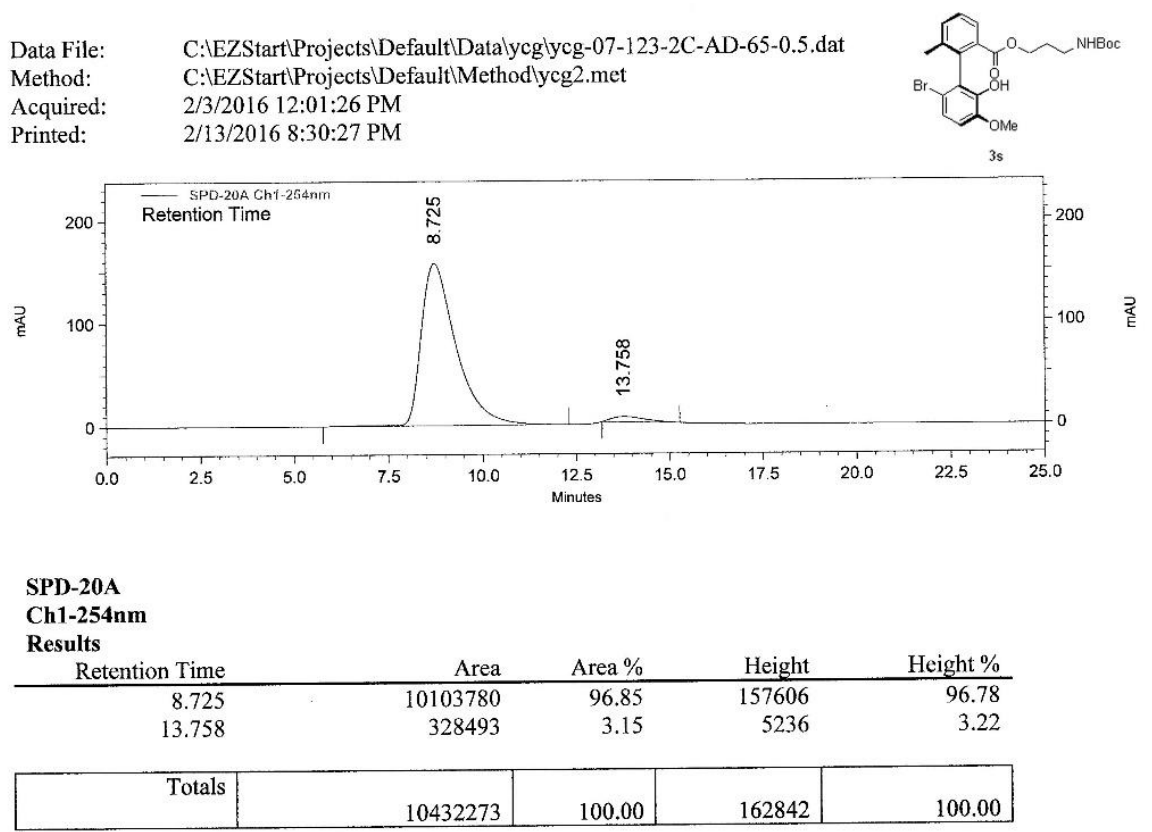




\section{University of New Mexico}

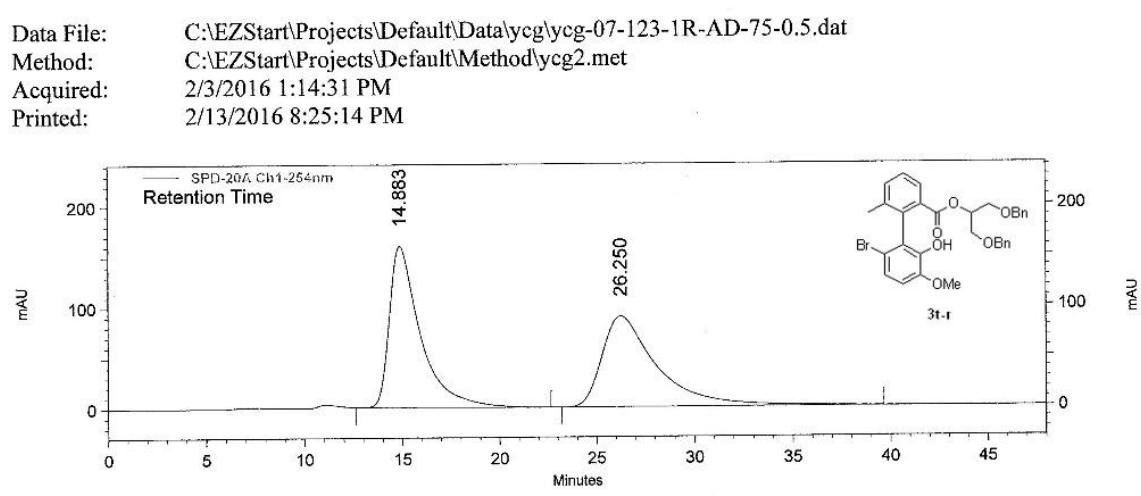

SPD-20A

Ch1-254nm

Results

\begin{tabular}{rrrrrr}
$\begin{array}{c}\text { Results } \\
\text { Retention Time }\end{array}$ & Area & Area \% & Height & Height \% \\
\hline 14.883 & 17743677 & 50.32 & 159496 & 63.97 \\
26.250 & 17516929 & 49.68 & 89832 & 36.03 \\
\hline Totals & 35260606 & 100.00 & 249328 & 100.00 \\
\hline
\end{tabular}

\section{University of New Mexico}
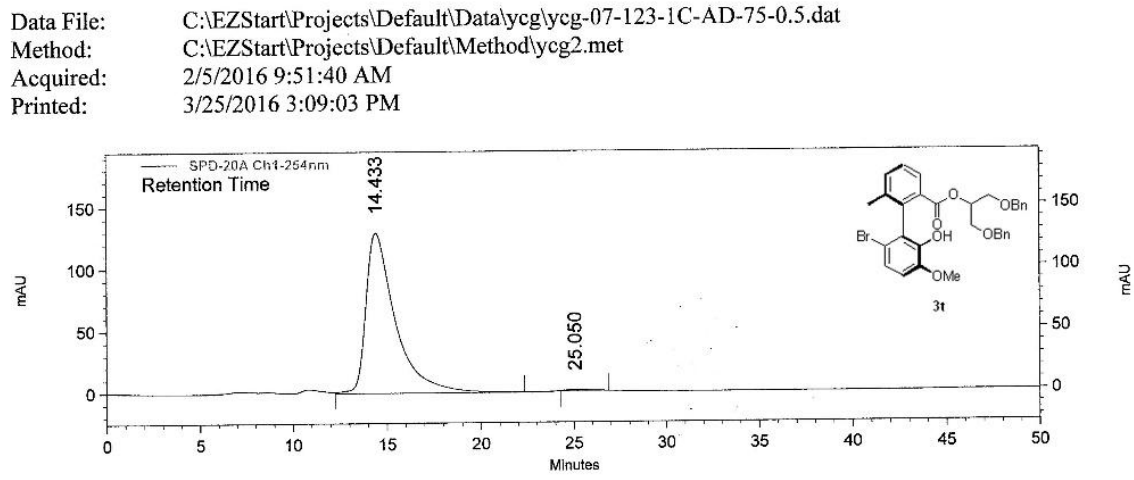

SPD-20A

Ch1-254nm

Results

Retention Time

14.433

25.050

Area

Area \%

Height

Height \%

Totals 78655

884

0.68

\begin{tabular}{|r|r|r|r|r|}
\hline Totals & 14199131 & 100.00 & 130221 & 100.00 \\
\hline
\end{tabular}




\section{University of New Mexico}

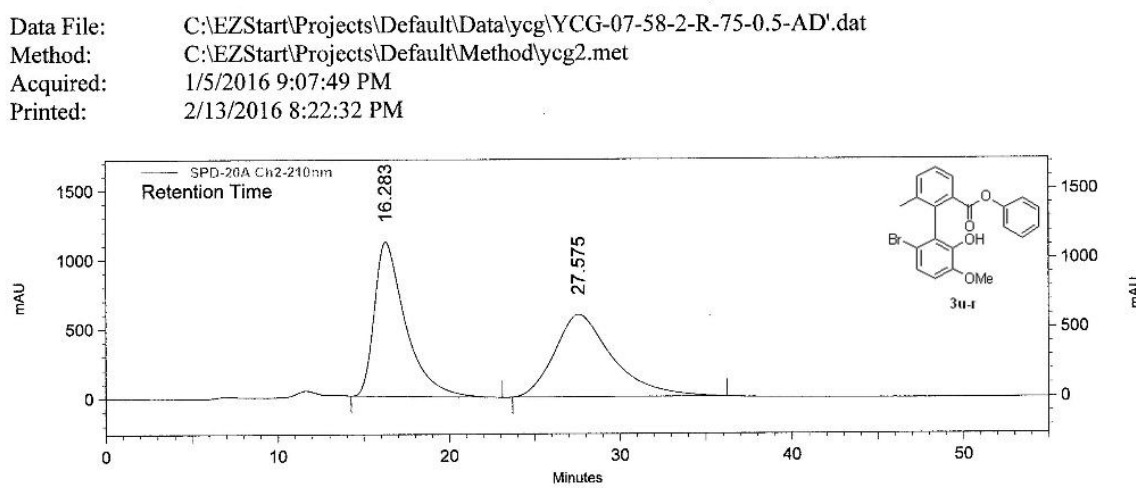

SPD-20A

Ch2-210nm

Results

Retention Time

16.283

27.575

Area \%

\begin{tabular}{rrr}
$\%$ & Height & Height \% \\
\hline .30 & 1113436 & 65.22 \\
.70 & 593691 & 34.78
\end{tabular}

Totals 139243036

$49.70 \quad 59369$

\begin{tabular}{|r|r|r|r|r|}
\hline Totals & 280146906 & 100.00 & 1707127 & 100.00 \\
\hline
\end{tabular}

\section{University of New Mexico}
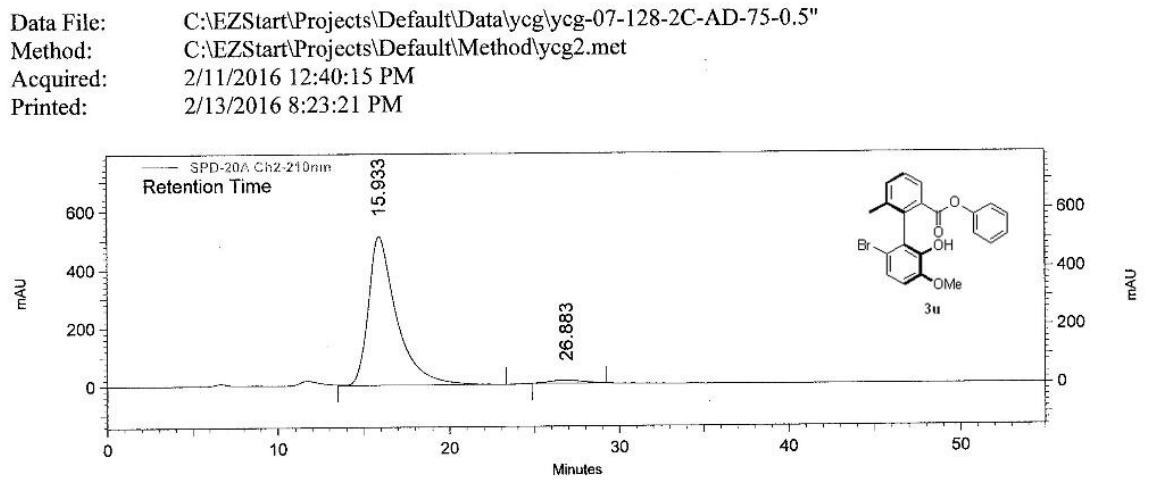

SPD-20A

Ch2-210nm

Results

Retention Time

15.933

Area

Area \% Height

$510590 \quad$ Height \%

26.883

$\begin{array}{rrrr}58047190 & 97.73 & 510590 & 98.07 \\ 1347604 & 2.27 & 10071 & 1.93\end{array}$

Totals

$59394794 \quad 100.00 \quad 520661$

100.00 


\section{University of New Mexico}

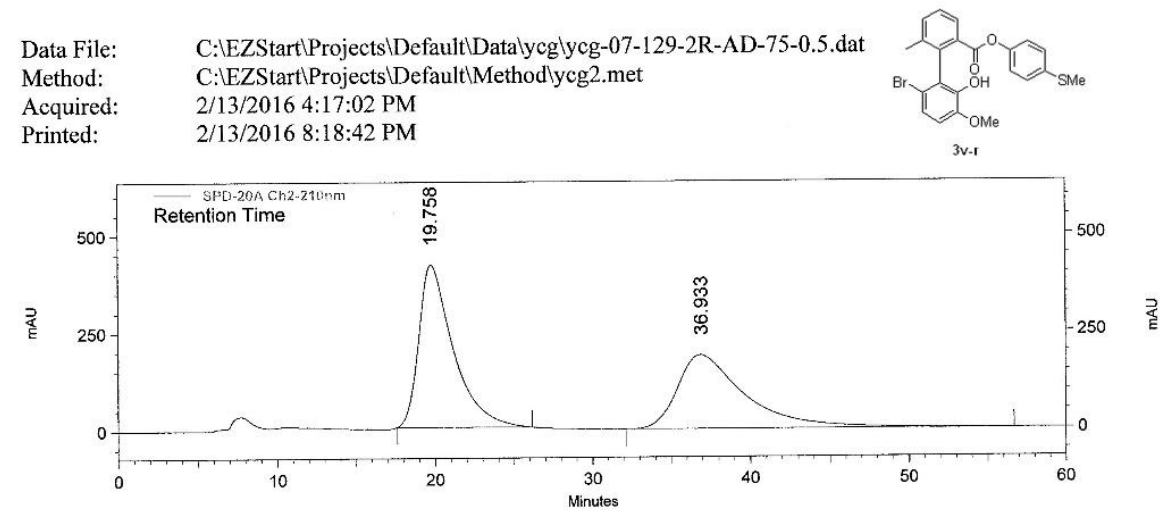

SPD-20A

Ch2-210nm

Results

Retention Time

19.758

36.933

\begin{tabular}{rrrr} 
Area & Area \% & Height & Height \% \\
\hline 63228206 & 53.04 & 416046 & 68.78 \\
55987881 & 46.96 & 188881 & 31.22
\end{tabular}

Totals

\begin{tabular}{|c|r|r|r|}
119216087 & 100.00 & 604927 & 100.00 \\
\hline
\end{tabular}

\section{University of New Mexico}

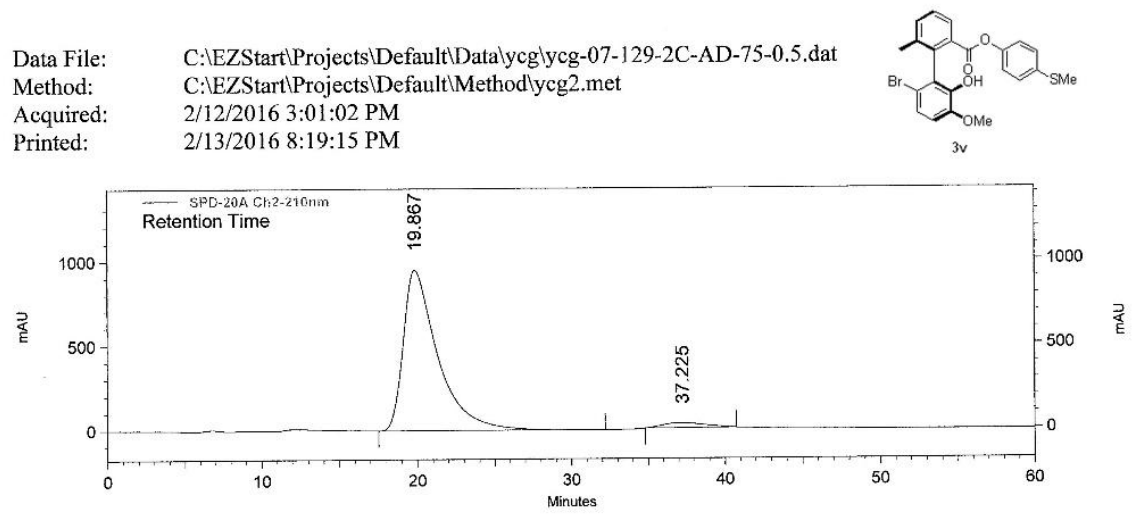

SPD-20A

Ch2-210nm

Results

Retention Time

\begin{tabular}{rrrrr} 
Time & Area & Area \% & Height & Height \% \\
\hline 19.867 & 147176935 & 96.39 & 950628 & 96.99 \\
37.225 & 5517696 & 3.61 & 29524 & 3.01
\end{tabular}

Totals

152694631

100.00

100.00 


\section{University of New Mexico}

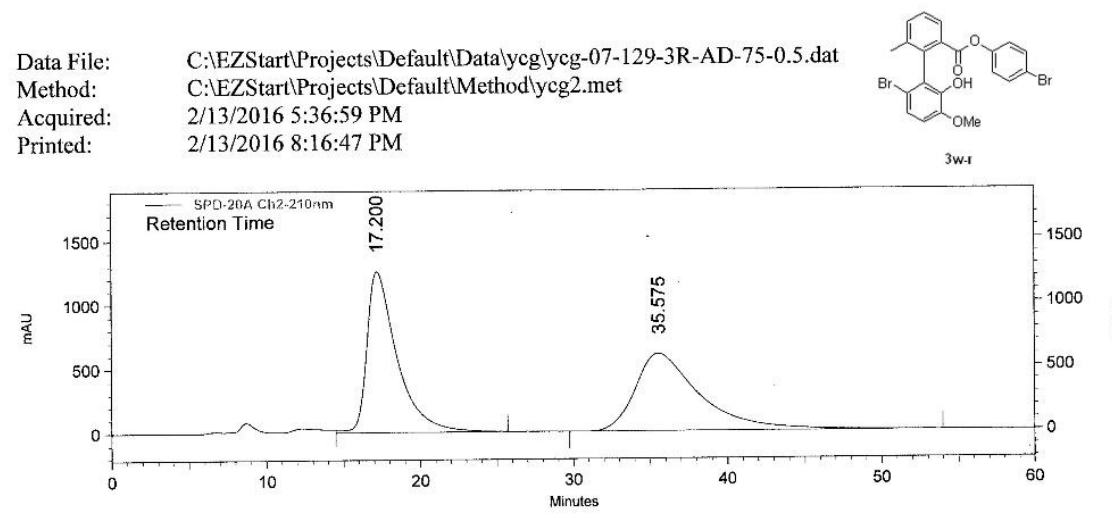

SPD-20A

Ch2-210nm

Results

Retention Time

17.200

35.575

\begin{tabular}{rrrr} 
Area & Area \% & Height & Height \% \\
\hline 72532107 & 49.59 & 1253163 & 67.53 \\
75395111 & 50.41 & 602516 & 32.47
\end{tabular}

\begin{tabular}{|r|r|r|r|r|}
\hline Totals & 347927218 & 100.00 & 1855679 & 100.00 \\
\hline
\end{tabular}

\section{University of New Mexico}

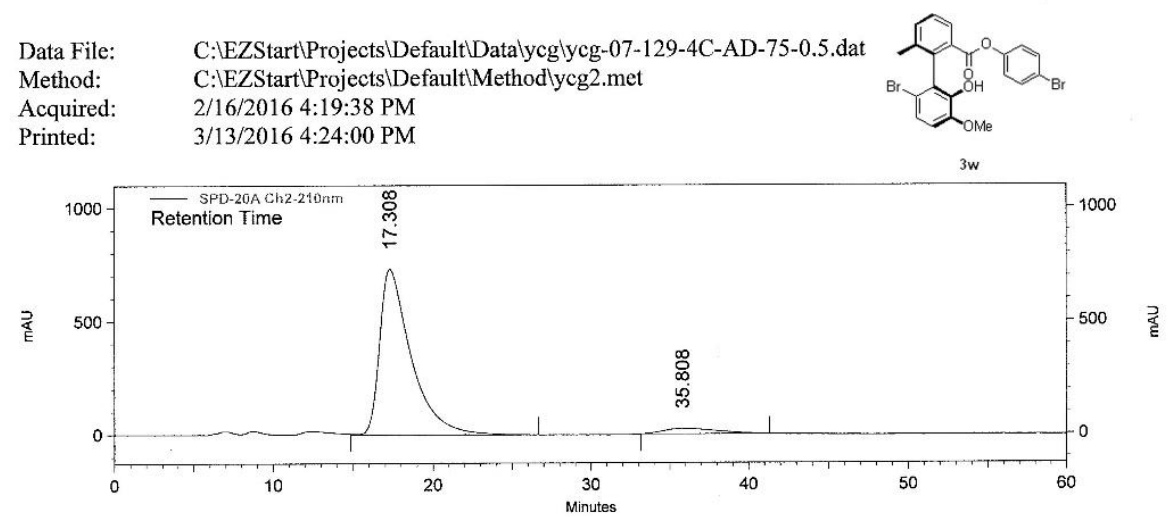

SPD-20A

Ch2-210nm

Results

Retention Time

-17.308
-35.808

Area

Area \%

Height

Height \%

35.808

5558284

732192
23958

96.83

Totals

106372409

756150

100.00 


\section{University of New Mexico}

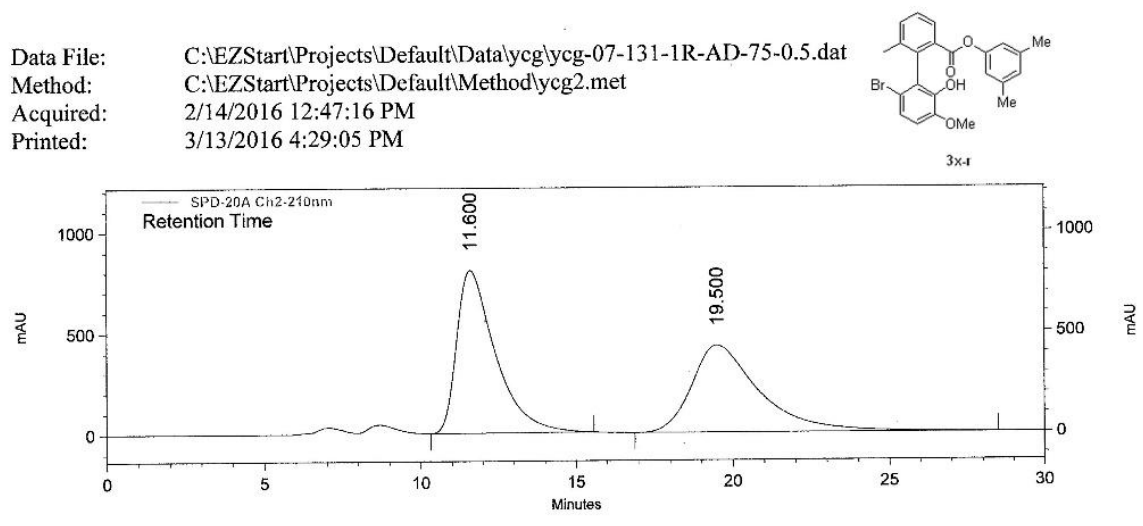

SPD-20A

Ch2-210nm

Results

\begin{tabular}{rrrrrr}
$\begin{array}{c}\text { Results } \\
\text { Retention Time }\end{array}$ & Area & Area \% & Height & Height \% \\
\hline 11.600 & 70028451 & 51.98 & 807151 & 65.16 \\
19.500 & 64705438 & 48.02 & 431586 & 34.84 \\
\hline Totals & 134733889 & 100.00 & 1238737 & 100.00 \\
\hline
\end{tabular}

\section{University of New Mexico}

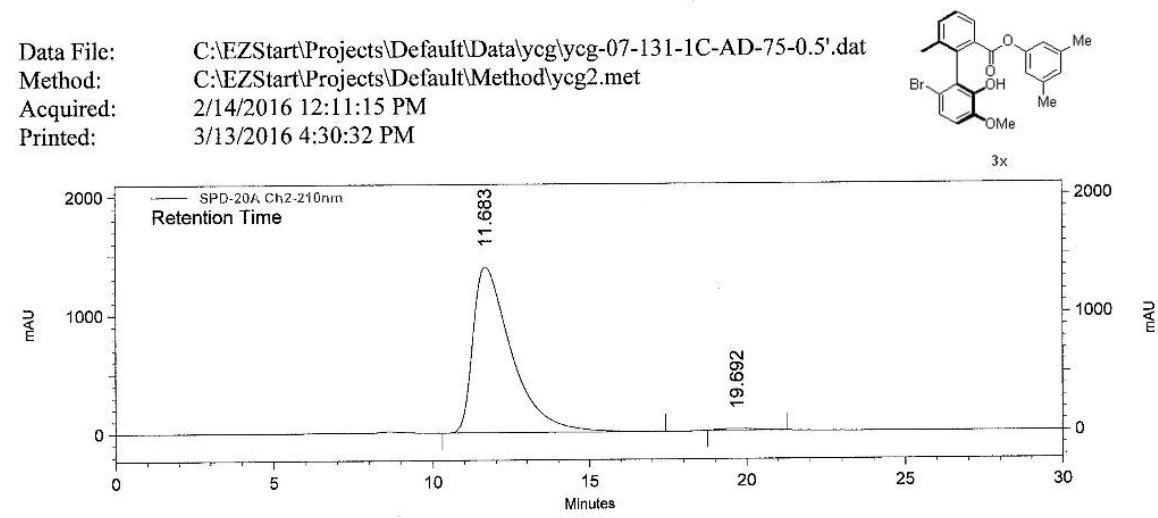

SPD-20A

Ch2-210nm

Results

\begin{tabular}{rrrrrr|}
$\begin{array}{r}\text { Results } \\
\text { Retention Time }\end{array}$ & Area & Area \% & Height & Height \% \\
\hline 11.683 & 119897055 & 99.12 & 1396859 & 99.10 \\
19.692 & 1068357 & 0.88 & 12679 & 0.90 \\
\hline \multicolumn{2}{r|}{ Totals } & 120965412 & 100.00 & 1409538 & 100.00 \\
\hline
\end{tabular}




\section{University of New Mexico}

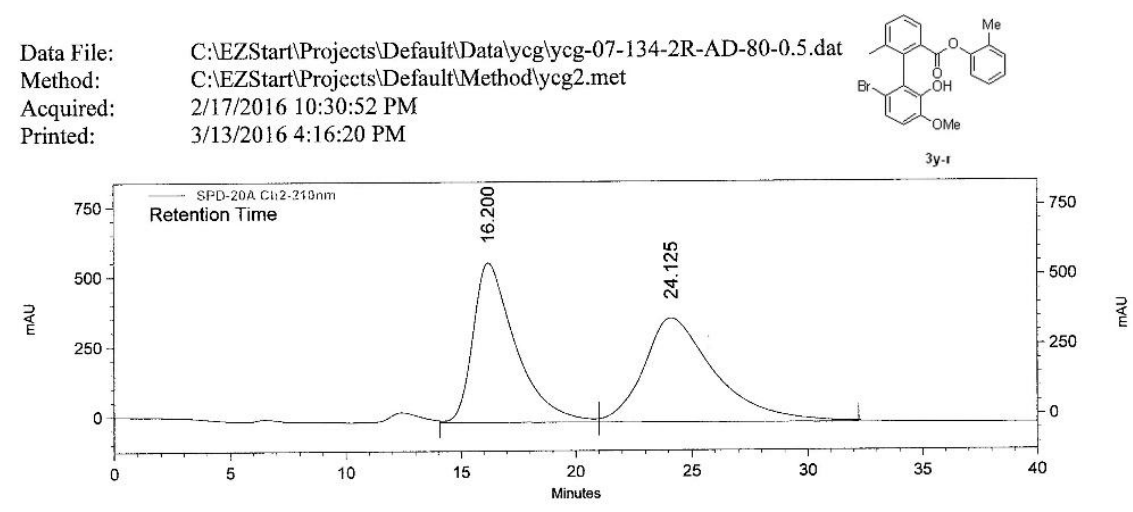

SPD-20A

Ch2-210nm

Results

\begin{tabular}{rrrrrr|}
$\begin{array}{c}\text { Area } \\
\text { Retention Time }\end{array}$ & Area \% & Height & Height \% \\
\hline 16.200 & 74149897 & 48.34 & 571677 & 60.60 \\
24.125 & 79247108 & 51.66 & 371672 & 39.40 \\
\multicolumn{2}{l}{ Totals } & 153397005 & 100.00 & 943349 & 100.00 \\
\hline
\end{tabular}

\section{University of New Mexico}

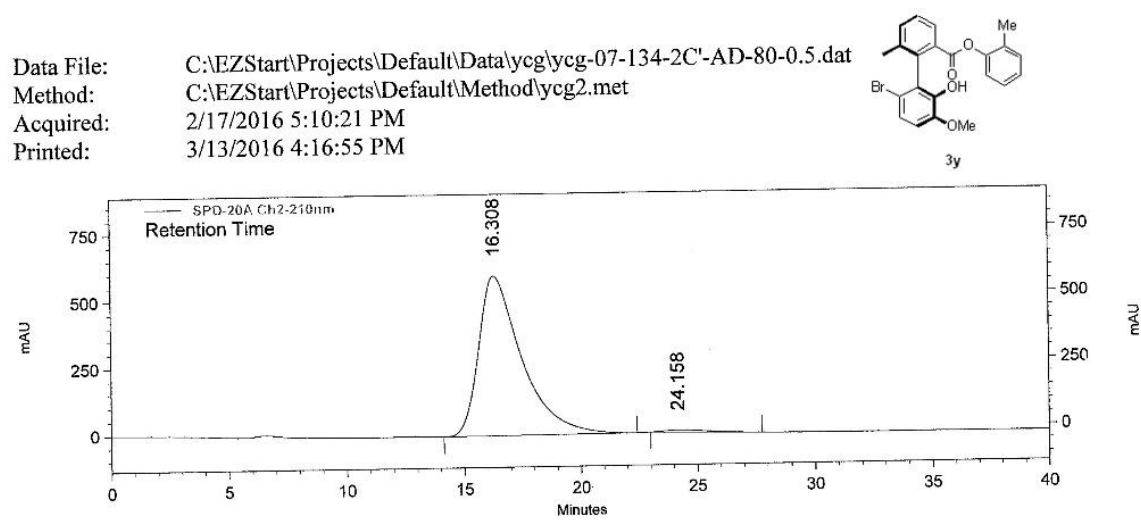

SPD-20A

Ch2-210nm

Results

Retention Time

16.308

Area Area \%

Height Height \%

24.158

76937525

98.65
1.35

$598990 \quad 98.69$

Totals

$77988540 \quad 100.00$

606960

100.00 


\section{University of New Mexico}

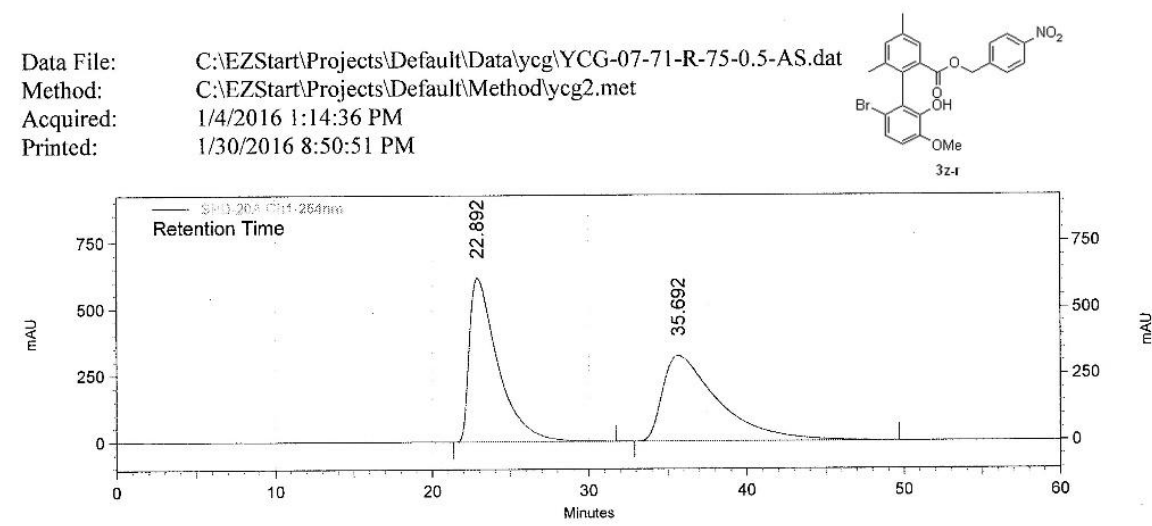

SPD-20A

Ch1-254nm

Results

\begin{tabular}{rrrrrr}
$\begin{array}{r}\text { Results } \\
\text { Retention Time }\end{array}$ & Area \% & Height & Height \% \\
\hline 22.892 & 78401002 & 50.09 & 616488 & 65.83 \\
35.692 & 78113437 & 49.91 & 320047 & 34.17 \\
\hline \multicolumn{2}{|c|}{ Totals } & 156514439 & 100.00 & 936535 & 100.00 \\
\hline
\end{tabular}

\section{University of New Mexico}

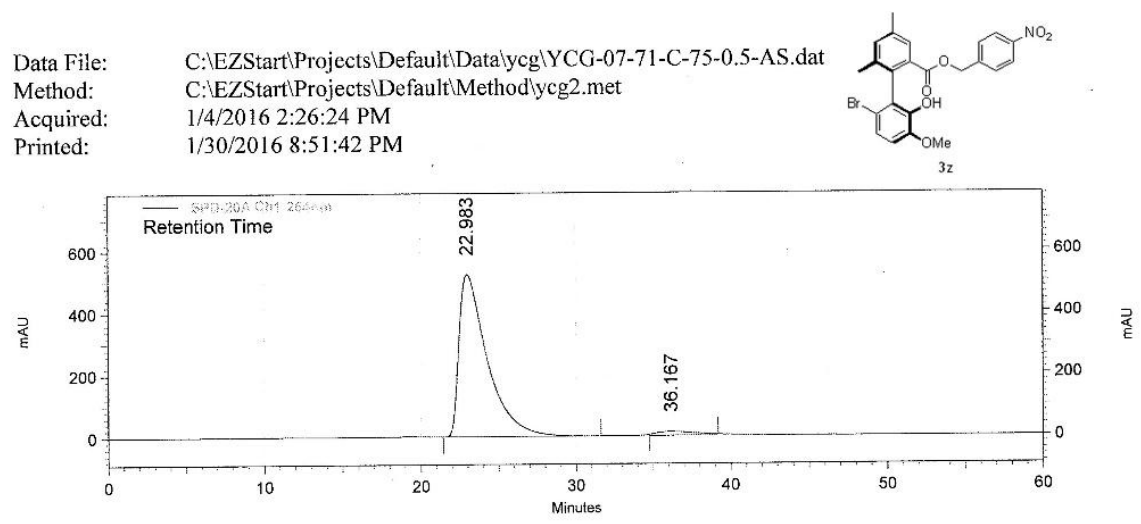

SPD-20A

Ch1-254nm

Results

\begin{tabular}{rrrrrr|}
$\begin{array}{c}\text { Results } \\
\text { Retention Time }\end{array}$ & Area & Area \% & Height & Height \% \\
\hline 22.983 & 66047701 & 96.92 & 522675 & 97.75 \\
36.167 & 2097605 & 3.08 & 12036 & 2.25 \\
\hline \multicolumn{2}{|c|}{ Totals } & 68145306 & 100.00 & 534711 & 100.00 \\
\hline
\end{tabular}




\section{University of New Mexico}

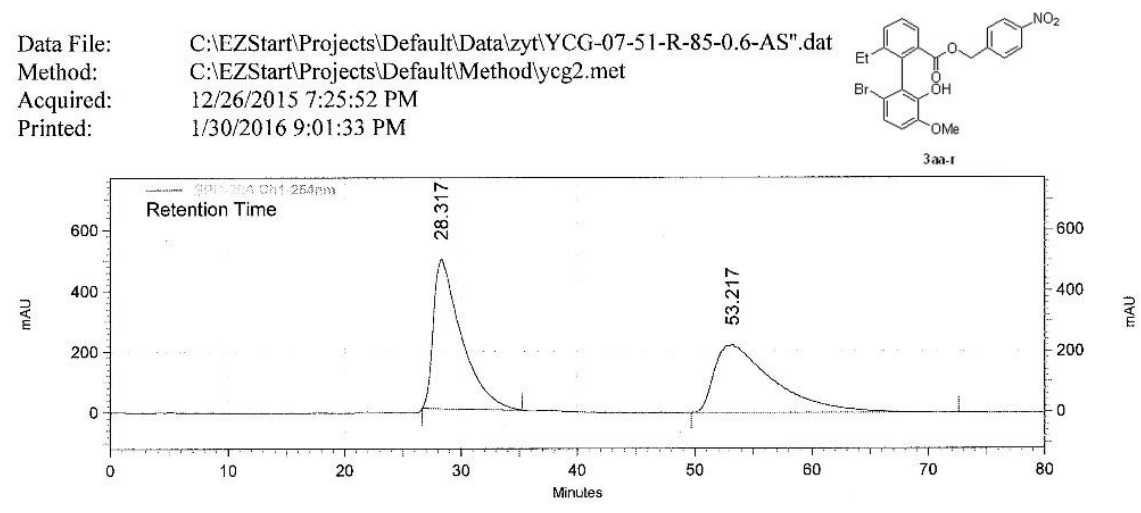

SPD-20A

Ch1-254nm

Results Retention Time 28.317 53.217

\begin{tabular}{rrrr} 
Area & Area \% & Height & Height \% \\
\hline 82298381 & 51.40 & 492031 & 68.78 \\
77819489 & 48.60 & 223356 & 31.22
\end{tabular}

Totals 100.00 715387

\section{University of New Mexico}

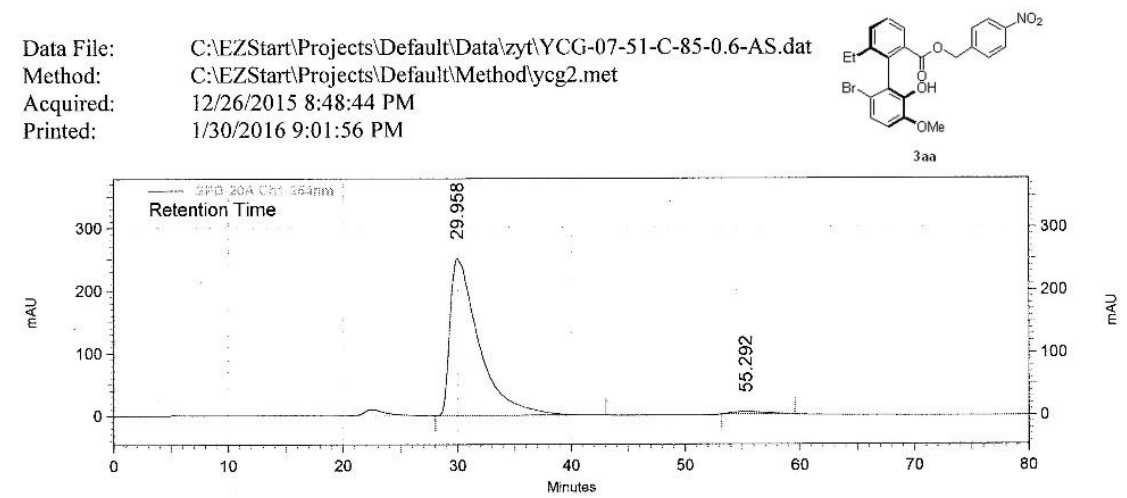

SPD-20A

Ch1-254nm

Results

Retention Time

29.958
55.292

\begin{tabular}{rrrr} 
Area & Area \% & Height & Height \% \\
\hline 44719666 & 98.26 & 250934 & 98.51 \\
792727 & 1.74 & 3799 & 1.49
\end{tabular}

Totals

45512393

100.00

254733 


\section{University of New Mexico}

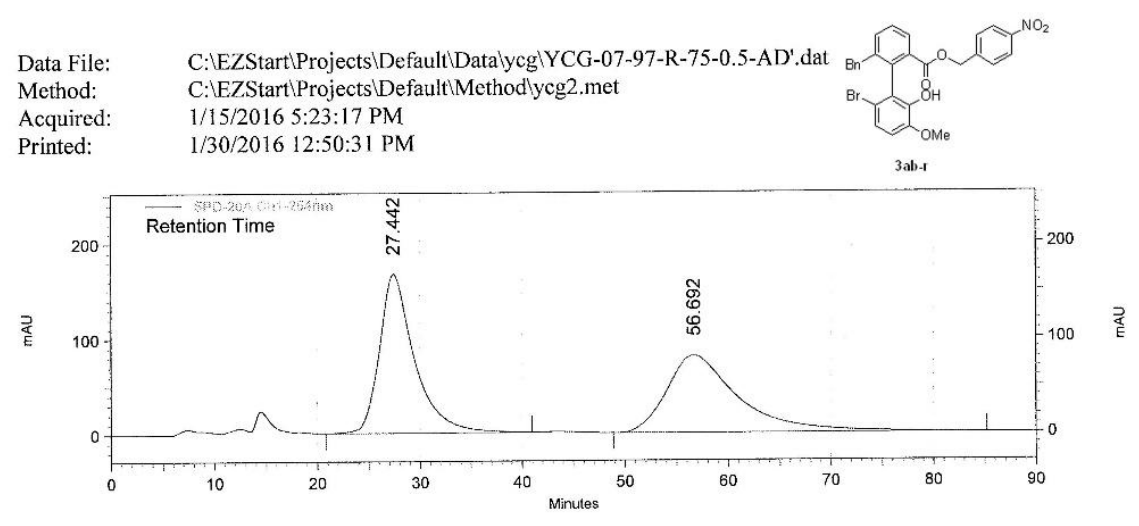

SPD-20A

Ch1-254nm

Results

\begin{tabular}{rrrrrr}
$\begin{array}{c}\text { Results } \\
\text { Retention Time }\end{array}$ & Area & Area \% & Height & Height \% \\
\hline 27.442 & 39870426 & 50.61 & 166902 & 67.36 \\
56.692 & 38916808 & 49.39 & 80861 & 32.64 \\
\hline Totals & 78787234 & 100.00 & 247763 & 100.00 \\
\hline
\end{tabular}

\section{University of New Mexico}

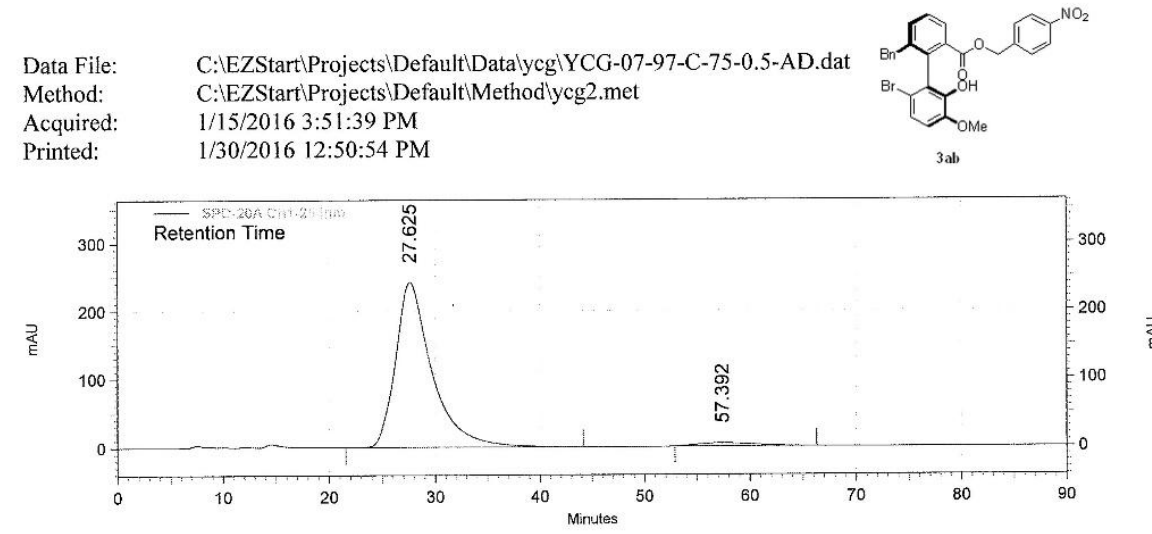

SPD-20A

Ch1-254nm

Results

Retention Time

27.625

57.392

Area

Area \%

Height

241880

Height \%

$\begin{array}{rrrr}57979301 & 97.25 & 241880 & 98.21 \\ 1637721 & 2.75 & 4418 & 1.79\end{array}$

Totals

\begin{tabular}{|l|r|r|}
\hline 59617022 & 100.00 & 246298 \\
\hline
\end{tabular}

100.00 


\section{University of New Mexico}

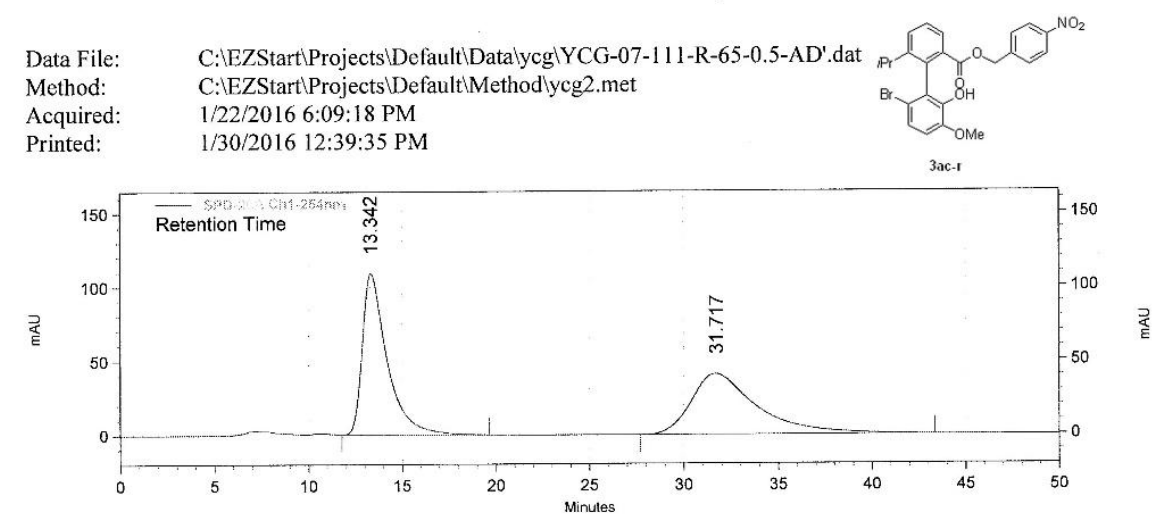

SPD-20A

Ch1-254nm

Results

\begin{tabular}{rrrrr}
$\begin{array}{l}\text { Results } \\
\text { Retention Time }\end{array}$ & Area & Area \% & Height & Height \% \\
\hline 13.342 & 9996535 & 50.40 & 108884 & 72.71 \\
31.717 & 9836499 & 49.60 & 40871 & 27.29 \\
& & & & \\
\hline
\end{tabular}

\begin{tabular}{|r|r|r|r|r|}
\hline Totals & 19833034 & 100.00 & 149755 & 100.00 \\
\hline
\end{tabular}

\section{University of New Mexico}

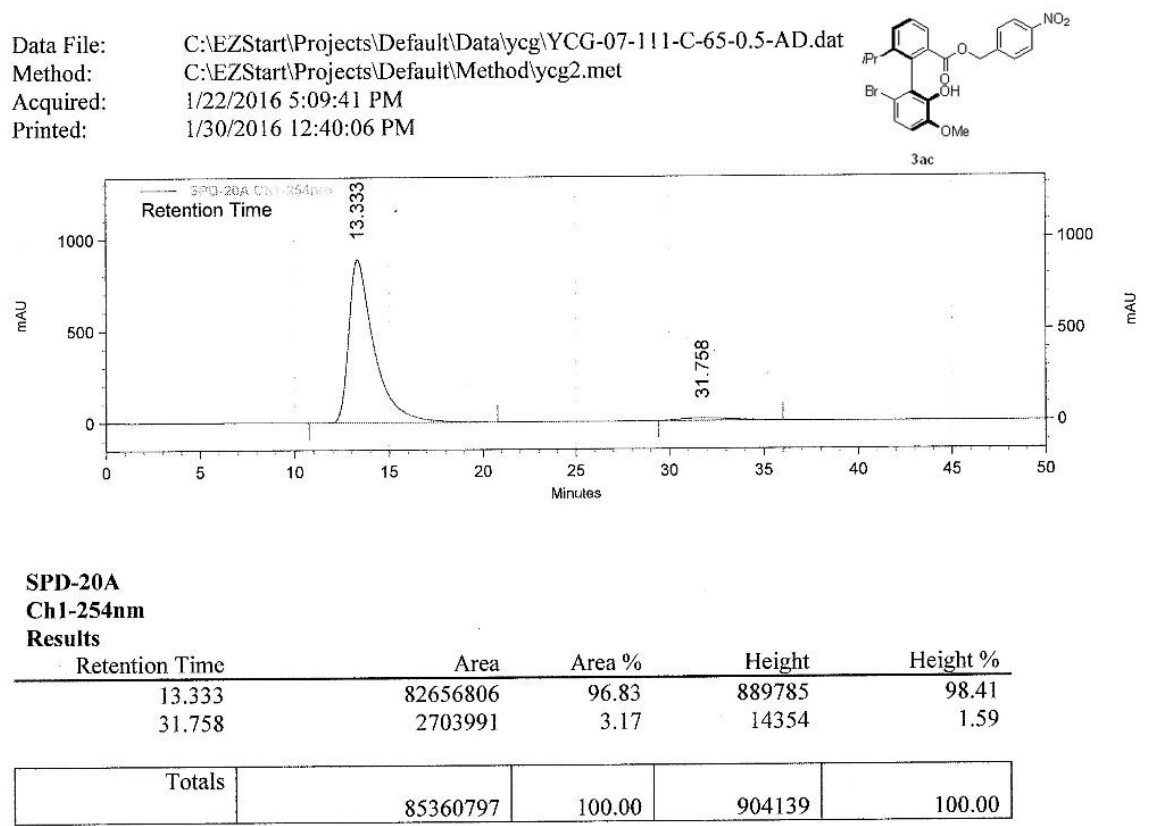




\section{University of New Mexico}

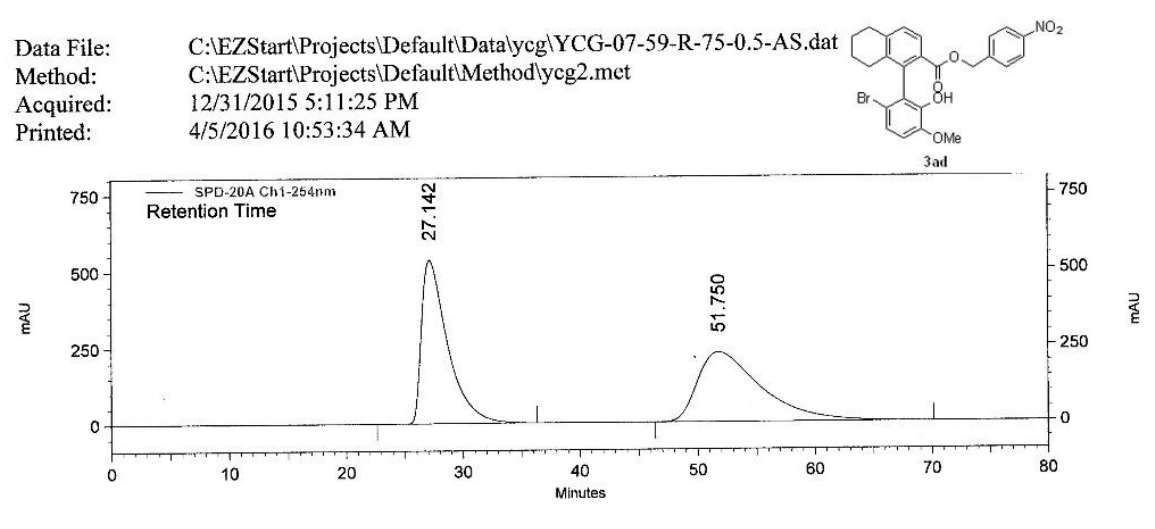

SPD-20A

Ch1-254nm

Resuits

Retention Time

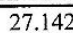

27.142
51.750

\begin{tabular}{rr} 
Area & Area \% \\
\hline 84165787 & 49.63
\end{tabular}

\begin{tabular}{rrr} 
rea $\%$ & Height & Height \% \\
\hline 49.63 & 535862 & 70.14 \\
50.37 & 228138 & 29.86
\end{tabular}

Totals 85434365

100.00

764000

100.00

\section{University of New Mexico}

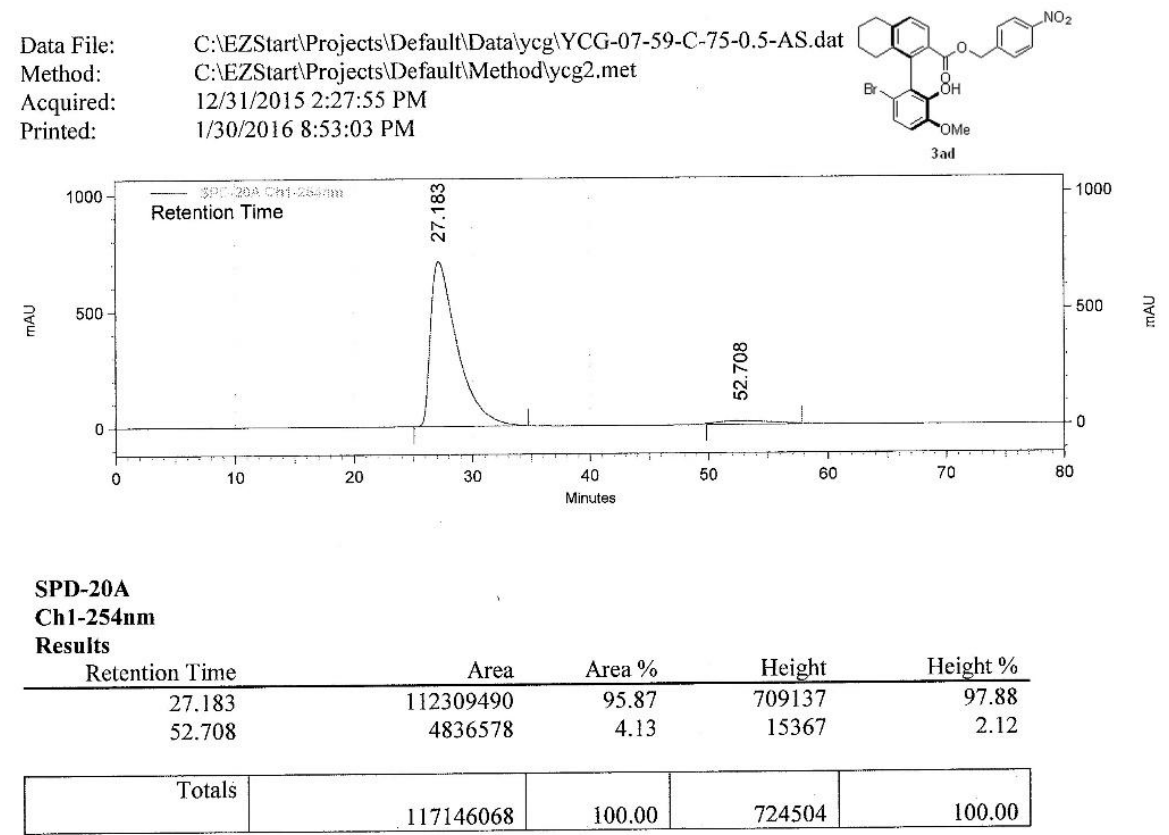




\section{University of New Mexico}

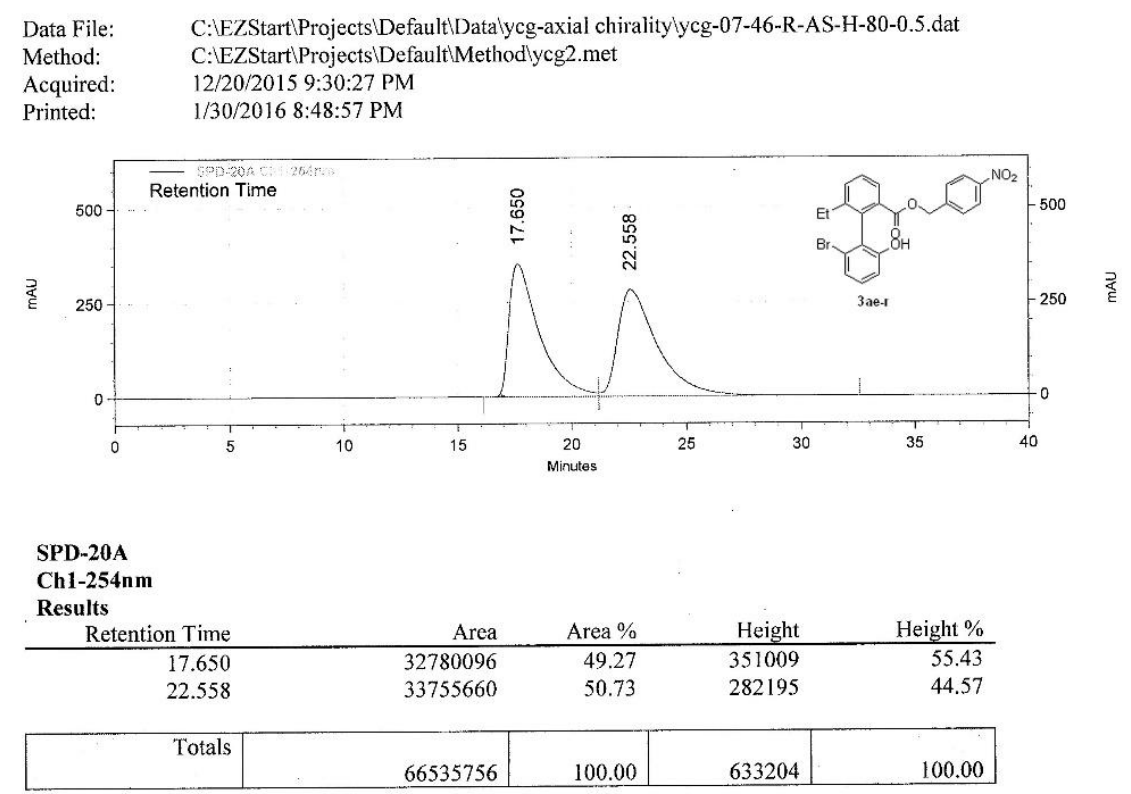

\section{University of New Mexico}
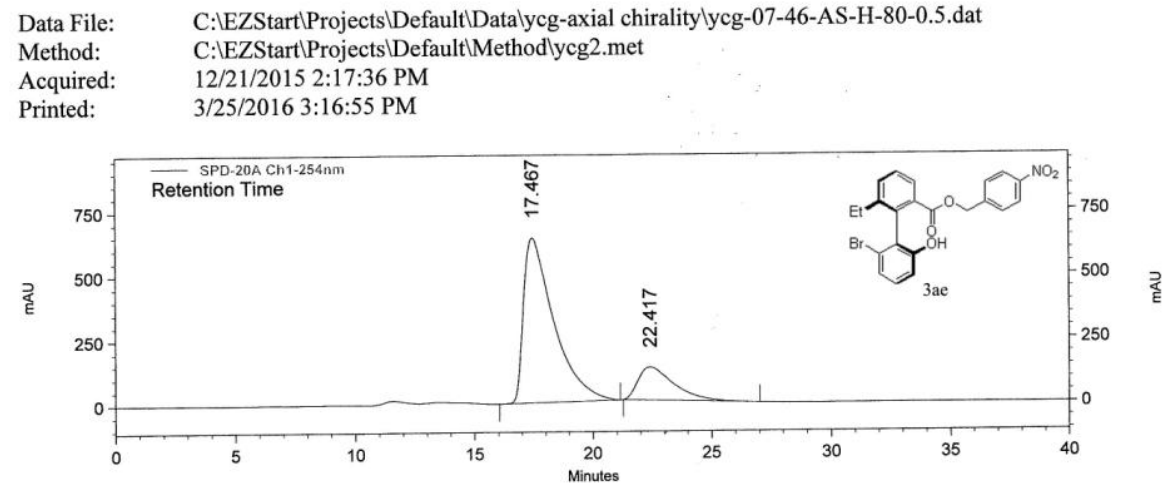

SPD-20A

Ch1-254nm

Results

Retention Time

17.467

22.417

Area

Area \%

80.88

19.12

Height

640922

Height \%

Totals

70947907

100.00

768980

100.00 


\section{University of New Mexico}

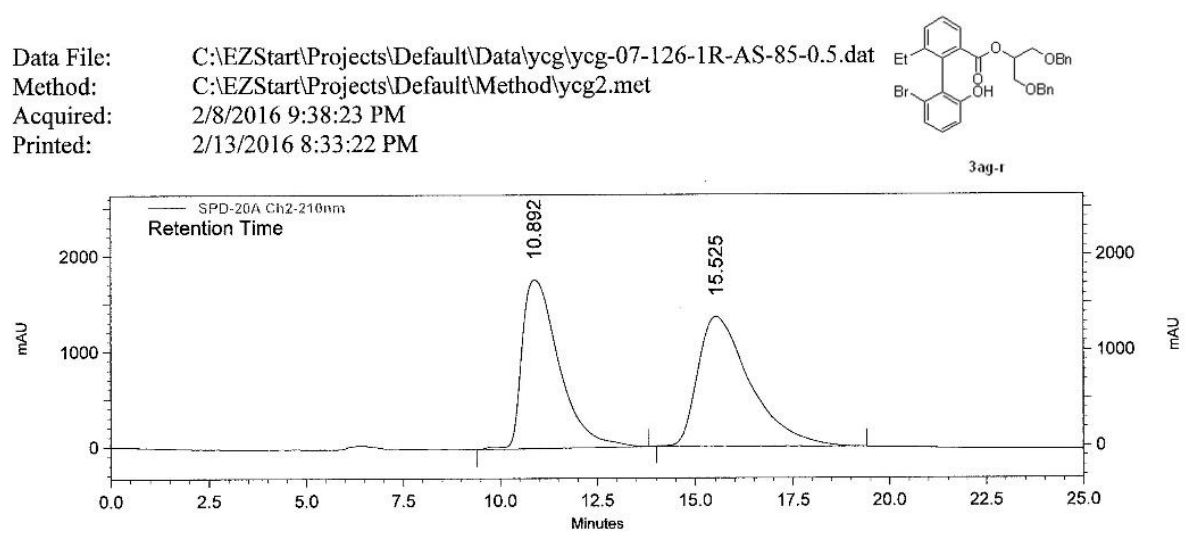

SPD-20A

Ch2-210nm

Results

Retention Time

10.892

Area Area \%

Height

1767698

15.525

48.38

1361596

43.51

\begin{tabular}{|r|r|r|r|r|}
\hline Totals & 243482199 & 100.00 & 3129294 & 100.00 \\
\hline
\end{tabular}

\section{University of New Mexico}

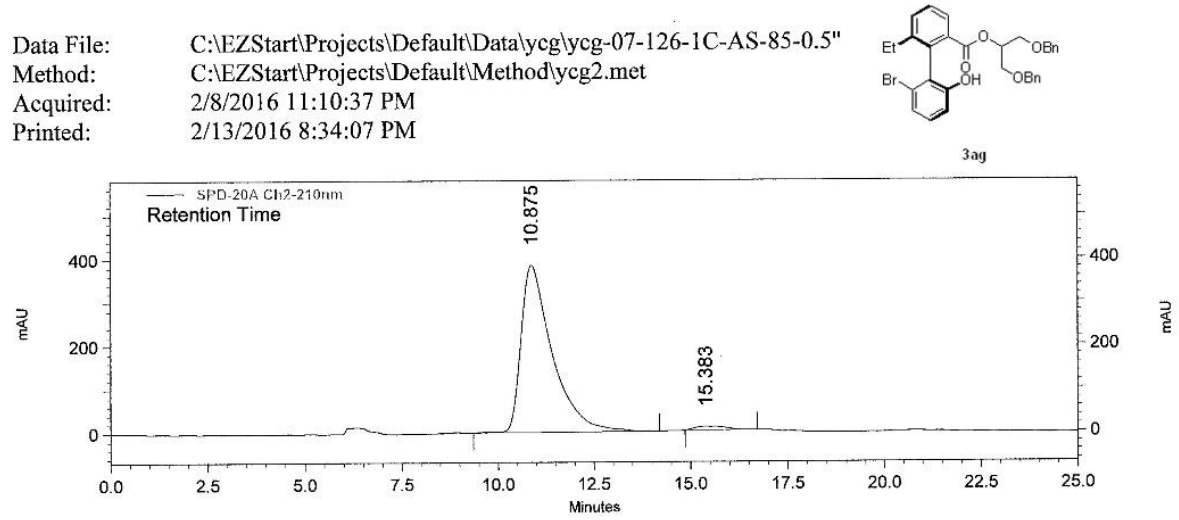

SPD-20A

Ch2-210nm

Results

Retention Time

10.875

Area

Area \%

384819

Height $\%$

15.383

454523

2.04

8228

2.09

Totals 


\section{University of New Mexico}

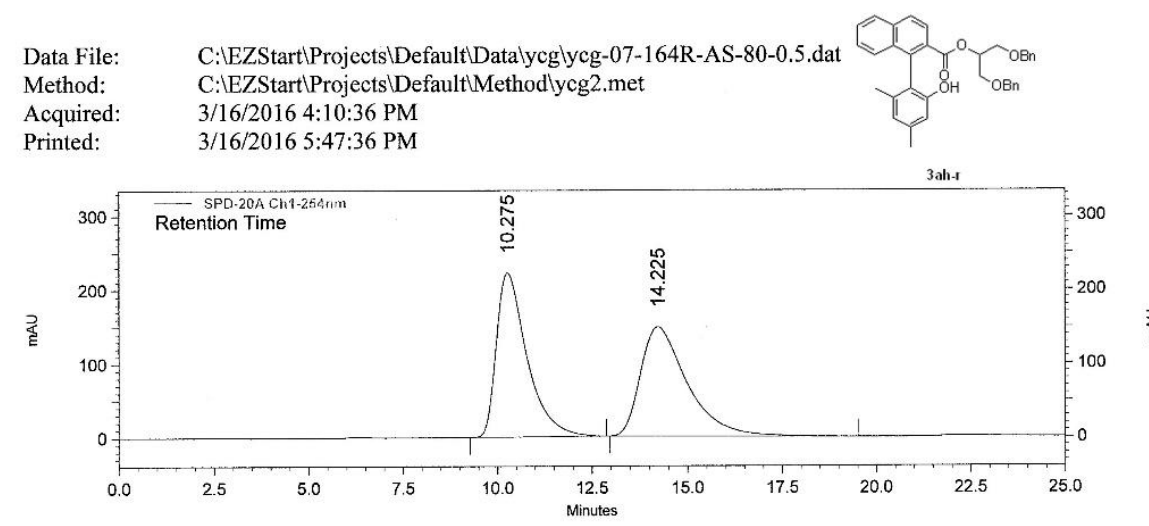

SPD-20A

Ch1-254nm

Results

Retention Time

10.275

Area Area \%

\begin{tabular}{rrr} 
rea \% & Height & Height \% \\
\hline 50.02 & 221778 & 59.94 \\
49.98 & 148215 & 40.06
\end{tabular}

\begin{tabular}{|r|r|r|r|r|}
\hline Totals & 25053118 & 100.00 & 369993 & 100.00 \\
\hline
\end{tabular}

\section{University of New Mexico}

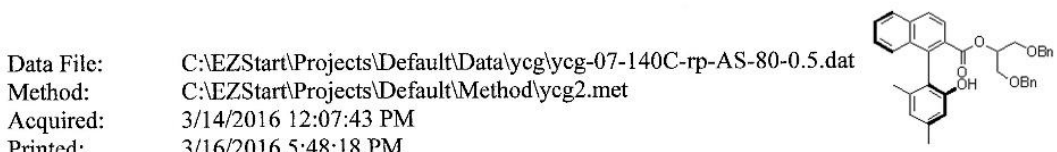

Printed:

3ah

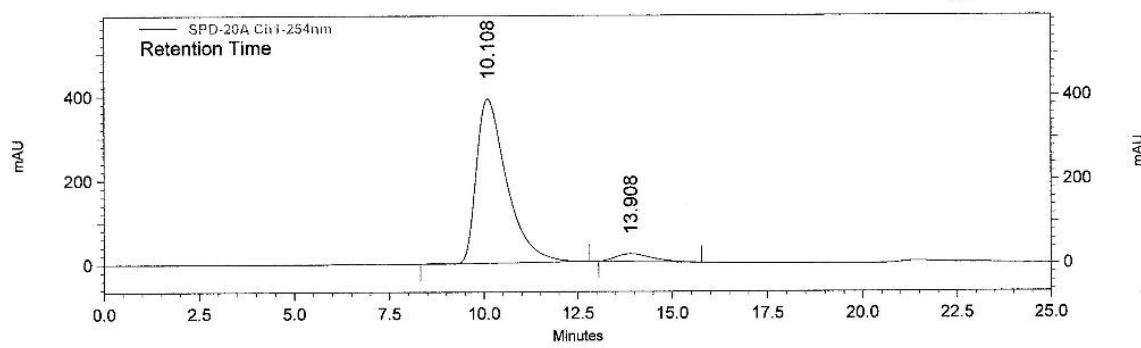

SPD-20A

Ch1-254nm

Results

\begin{tabular}{rrrrrr|} 
Retention Time & Area & Area \% & Height & Height \% \\
\hline 10.108 & 21989259 & 94.77 & 389954 & 95.47 \\
13.908 & 1212743 & 5.23 & 18523 & 4.53 \\
\hline \multicolumn{2}{|c|}{ Totals } & 23202002 & 100.00 & 408477 & 100.00 \\
\hline
\end{tabular}




\section{University of New Mexico}

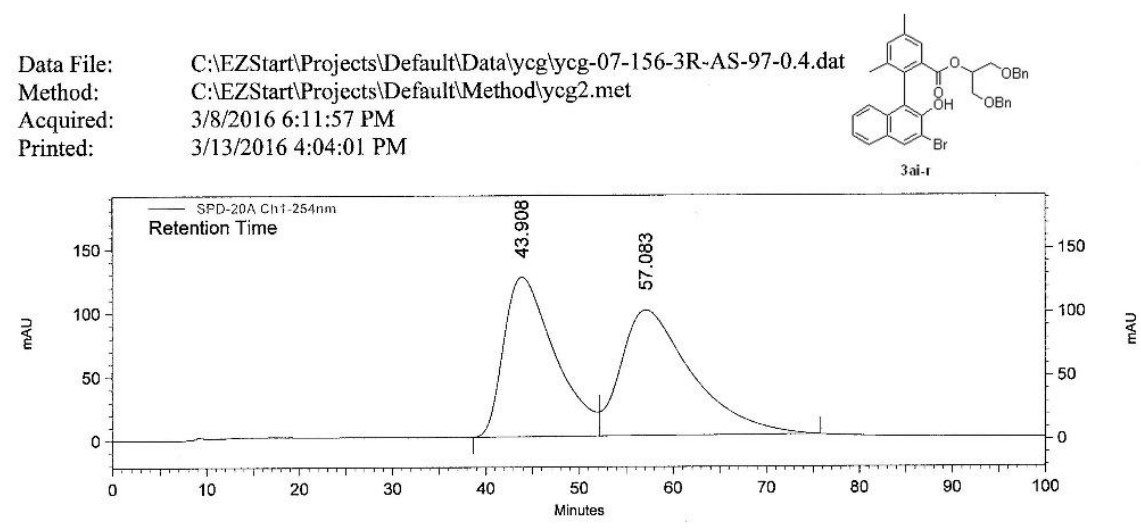

\section{SPD-20A}

Ch1-254nm

Results

\begin{tabular}{rrrrr} 
Retention Time & Area & Area \% & Height & Height \% \\
\hline 43.908 & 47484770 & 47.84 & 125125 & 55.98 \\
57.083 & 51766181 & 52.16 & 98386 & 44.02
\end{tabular}

57.083

5176618

52.

44.02

Totals

99250951

$100.00 \quad 223511$

100.00

\section{University of New Mexico}

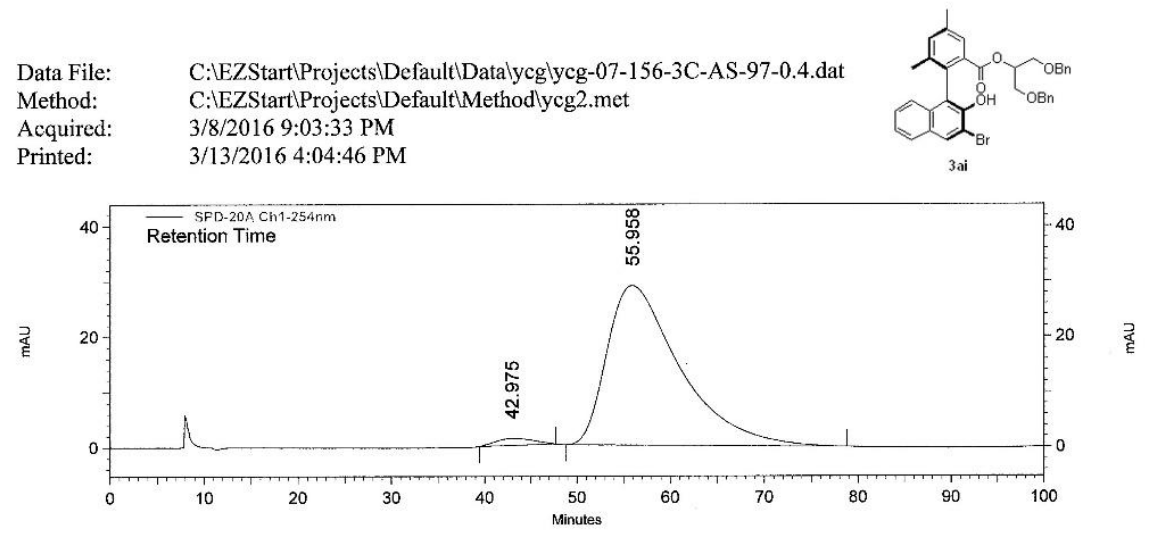

SPD-20A

Ch1-254nm

Results

Retention Time

42.975

55.958

Area \% Height

\begin{tabular}{rr} 
Height & Height \% \\
\hline 1295 & 4.29
\end{tabular}

Totals

\begin{tabular}{rrrr} 
Area & Area \% & Height & Height \% \\
\hline 357546 & 2.24 & 1295 & 4.29 \\
15628936 & 97.76 & 28868 & 95.71
\end{tabular}

15986482

30163

100.00 


\section{University of New Mexico}

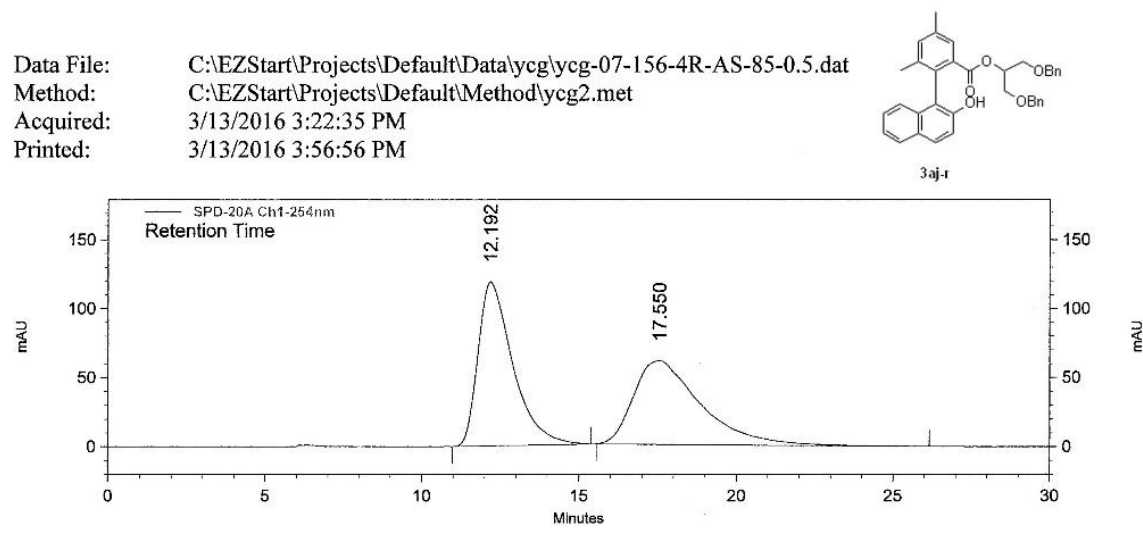

SPD-20A

Ch1-254nm

Results

\begin{tabular}{rrrrrr|}
$\begin{array}{c}\text { Results } \\
\text { Retention Time }\end{array}$ & Area & Area \% & Height & Height \% \\
\hline 12.192 & 9162411 & 50.48 & $\begin{array}{r}118809 \\
60536\end{array}$ & $\begin{array}{r}66.25 \\
17.550\end{array}$ \\
\hline Totals & 8987453 & 49.52 & & \\
\hline & 18149864 & 100.00 & 179345 & 100.00 \\
\hline
\end{tabular}

\section{University of New Mexico}

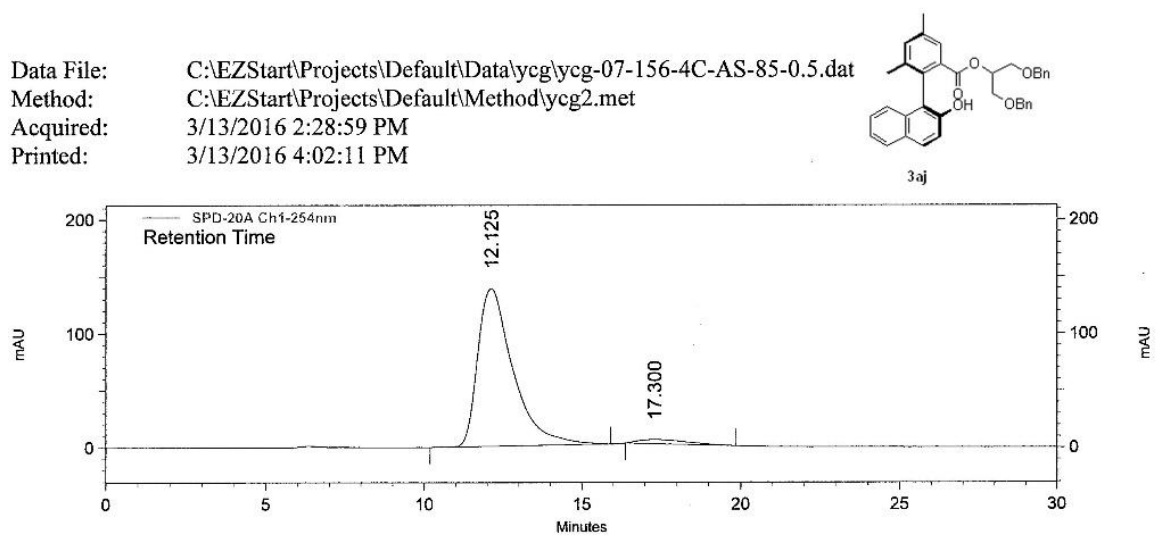

SPD-20A

Ch1-254nm

Results

Retention Time

12.125

17.300

Area

Area \%

$\begin{array}{rrr}96.53 & \text { Height } & \text { Height \% } \\ 3.47 & 37351 & 97.37\end{array}$

Totals

390128

142085

100.00 


\section{University of New Mexico}
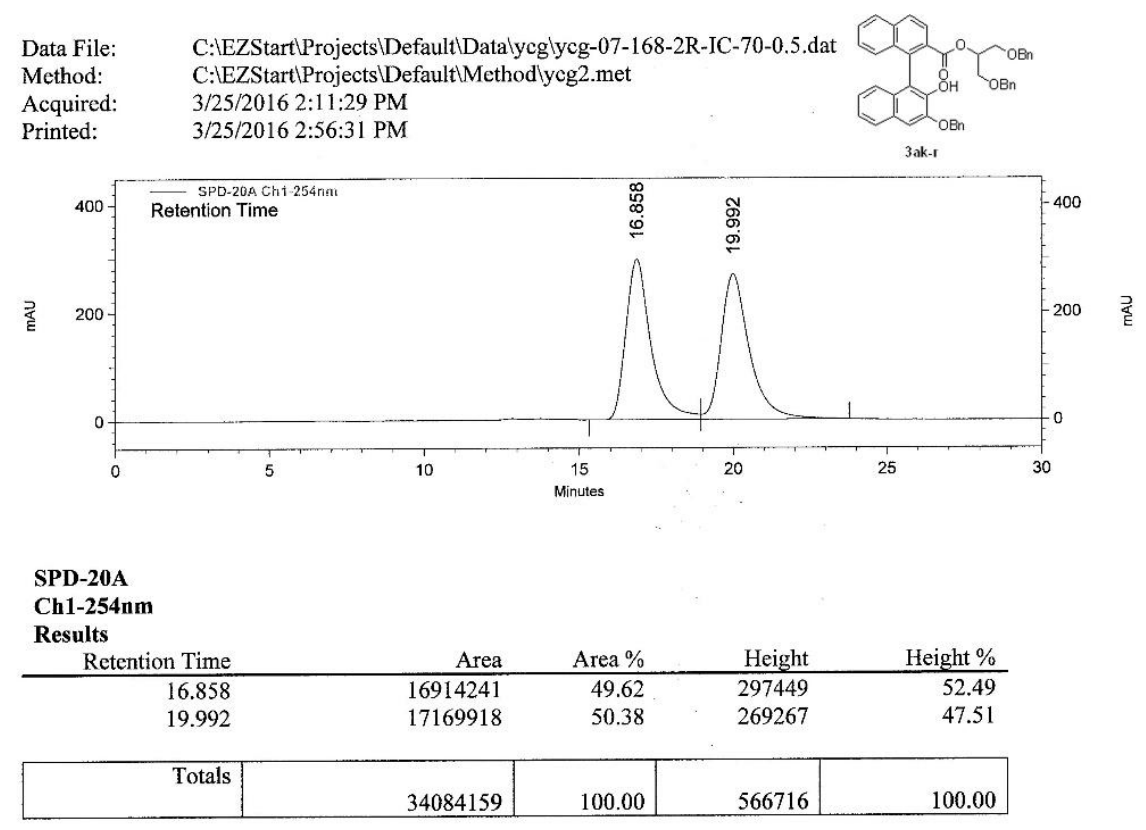

\section{University of New Mexico}

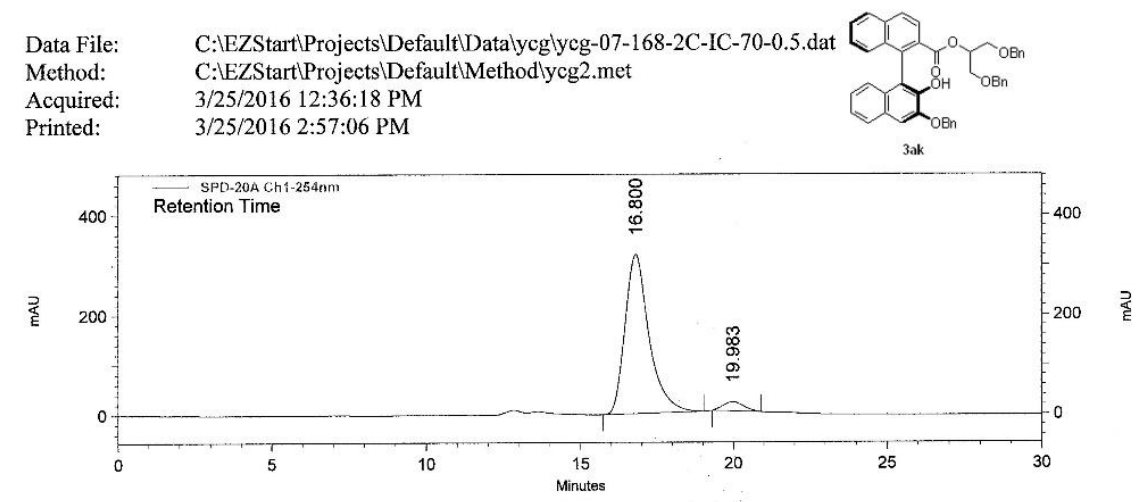

SPD-20A

Ch1-254nm

Results

Retention Time

16.800

19.983

\begin{tabular}{rrrr} 
Area & Area \% & Height & Height \% \\
\hline 17560011 & 95.43 & 319125 & 94.65 \\
840251 & 4.57 & 18055 & 5.35
\end{tabular}

Totals

337180

100.00 


\section{University of New Mexico}

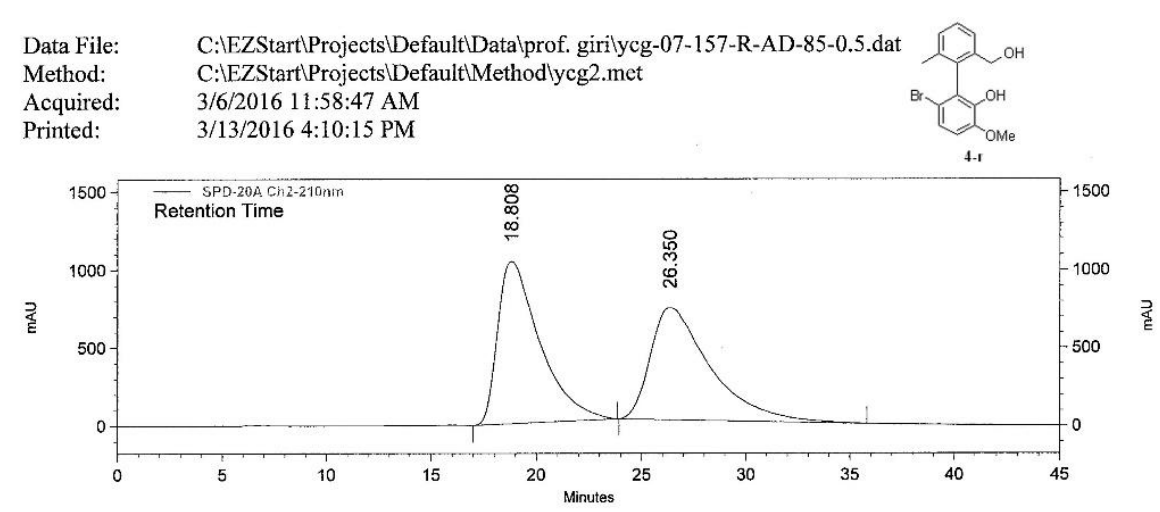

SPD-20A

Ch2-210nm

Results

\begin{tabular}{rrrrr}
$\begin{array}{l}\text { Results } \\
\text { Retention Time }\end{array}$ & Area & Area \% & Height & Height \% \\
\hline 18.808 & 143962527 & 50.15 & 1039559 & 59.17 \\
26.350 & 143108097 & 49.85 & 717467 & 40.83
\end{tabular}

\begin{tabular}{|r|r|r|r|r|}
\hline Totals & 287070624 & 100.00 & 1757026 & 100.00 \\
\hline
\end{tabular}

\section{University of New Mexico}

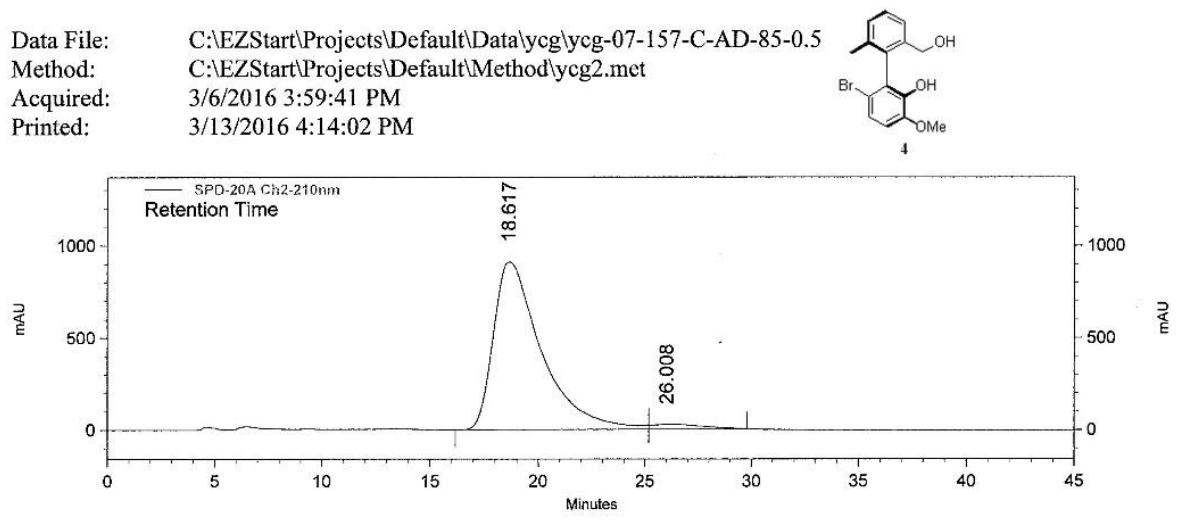

SPD-20A

Ch2-210nm

Results

\begin{tabular}{rrrrr}
$\begin{array}{c}\text { Results } \\
\text { Retention Time }\end{array}$ & Area & Area \% & Height & Height \% \\
\hline 18.617 & 140720953 & 97.05 & 909720 & 97.11 \\
26.008 & 4282996 & 2.95 & 27056 & 2.89
\end{tabular}

\begin{tabular}{|l|r|r|r|r|}
\hline Totals & 145003949 & 100.00 & 936776 & 100.00 \\
\hline
\end{tabular}




\section{University of New Mexico}

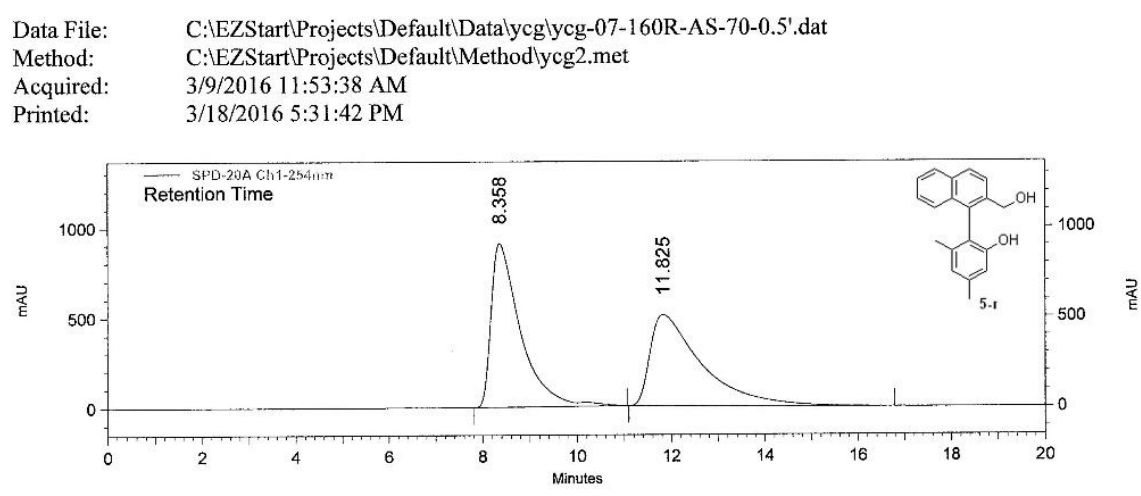

SPD-20A

Ch1-254nm

Results

Retention Time

\begin{tabular}{rrrrr} 
Time & Area & Area \% & Height & Height \% \\
\hline 8.358 & 39566153 & 50.87 & 909478 & 64.25 \\
11.825 & 38214771 & 49.13 & 505974 & 35.75
\end{tabular}

35.75

\begin{tabular}{|r|r|r|r|r|}
\hline Totals & 77780924 & 100.00 & 1415452 & 100.00 \\
\hline
\end{tabular}

\section{University of New Mexico}

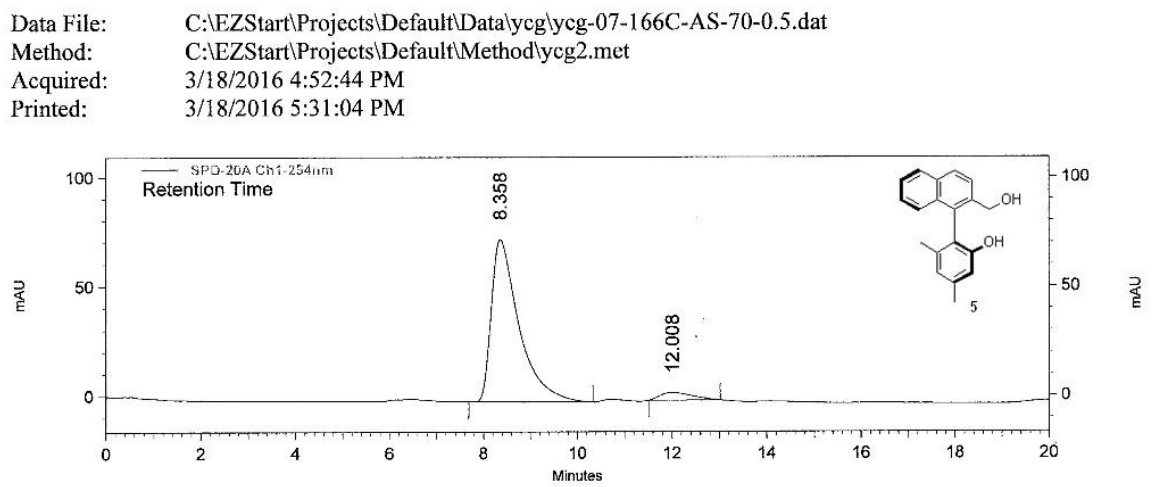

SPD-20A

Ch1-254nm

Results

Retention Time

8.358

8.358
12.008

\begin{tabular}{rrrr} 
Area & Area \% & Height & Height \% \\
\hline 3055466 & 94.83 & 74154 & 95.46 \\
166659 & 5.17 & 3523 & 4.54
\end{tabular}

Totals

\begin{tabular}{|l|l|l|l|}
\hline 3222125 & 100.00 & 77677 & 100.00 \\
\hline
\end{tabular}

